J. DIFFERENTIAL GEOMETRY

72 (2006) 169-338

\title{
MIRROR SYMMETRY VIA LOGARITHMIC DEGENERATION DATA I
}

\author{
Mark Gross \& Bernd Siebert
}

\begin{abstract}
This paper lays the foundations of a program to study mirror symmetry by studying the log structures of Illusie-Fontaine and Kato on degenerations of Calabi-Yau manifolds. The basic idea is that one can associate to certain sorts of degenerations of Calabi-Yau manifolds a log Calabi-Yau space, which is a $\log$ structure on the degenerate fibre. The log CY space captures essentially all the information of the degeneration, and hence all mirror statements for the "large complex structure limit" given by the degeneration can already be derived from the log CY space. In this paper we begin by discussing affine manifolds with singularities. Given such an affine manifold along with a polyhedral decomposition, we show how to construct a scheme consisting of a union of toric varieties. In certain non-degenerate cases, we can also construct log structures on these schemes. Conversely, given certain sorts of degenerations, one can build an affine manifold with singularities structure on the dual intersection complex of the degeneration. Mirror symmetry is then obtained as a discrete Legendre transform on these affine manifolds, thus providing an algebro-geometrization of the Strominger-Yau-Zaslow conjecture. The deepest result of this paper shows an isomorphism between $\log$ complex moduli of a $\log$ CY space and log Kähler moduli of its mirror.
\end{abstract}

\section{Contents}

Introduction

1. Affine Manifolds

1.1. Affine Manifolds and Invariants 177

1.2. Affine manifolds with singularities 183

1.3. Polyhedral decompositions 187

1.4. The discrete Legendre transform 202

1.5. Positivity and simplicity 208

This work was partially supported by NSF grant 0204326, EPSRC, the Heisenberg program of the DFG, and SPP 1094.

Received 06/03/2004. 
2. From polyhedral decompositions to algebraic spaces 218

2.1. The cone picture 219

2.2. The fan picture 222

3. Logarithmic structures 249

3.1. Introduction to $\log$ structures 249

3.2. Sheaves of log structures 255

3.3. Log structures for the fan picture 263

4. Toric degenerations 276

4.1. The dual intersection complex 280

4.2. Polarized log Calabi-Yau spaces and the intersection complex 287

4.3. Positive log structures $\quad 287$

4.4. Normalized gluing data and examples 290

5. Simplicity and mirror symmetry 298

5.1. Moduli of toric log Calabi-Yau spaces in the simple case 299

5.2. The logarithmic Picard group in the simple case 308

5.3. Mirror symmetry and conclusions 320

Appendix A. Simplicial and Polyhedral Complexes 323

A.1. Barycentric complexes 323

A.2. Polyhedral complexes $\quad 325$

A.3. Complexes of Sheaves $\quad 327$

Index of notations $\quad 330$

References 336

\section{Introduction}

This paper is the first arising from our project announced in [19] aiming at establishing a new paradigm for mirror symmetry. At the center of this approach is residual data associated to certain maximally unipotent degenerations $f: \mathcal{X} \rightarrow \mathcal{S}$ of Calabi-Yau varieties. The residual degeneration data consists of the central fibre $\mathcal{X}_{0}$ of the degeneration together with the log-structure induced from the inclusion $\mathcal{X}_{0} \subset \mathcal{X}$, a polarization, and an element of a "log Kähler moduli space". We claim that mirror symmetry comes down to an involution acting on residual degeneration data. In particular, degenerating families should be mirror-dual if and only if they have dual expressions in terms of residual data. The deepest result that we prove here is a basic duality between logarithmic complex and Kähler moduli of central fibres of degenerations as log spaces. Finer consequences for mirror symmetry will be addressed in a sequel to this paper. 
The central idea is to restrict attention to what we call toric degenerations of Calabi-Yau varieties (Definition 4.1). Roughly put, these are degenerations in which the singular fibre is a union of toric varieties and the map to the base is log smooth off of some bad set $Z$. In this context, we define a dual intersection complex capturing key data about the degeneration. The dual intersection complex we construct is an affine manifold $B$ with singularities together with a polyhedral decomposition $\mathscr{P}$. The affine structure depends on both the irreducible components of $\mathcal{X}_{0}$ and on information about the structure of $f$ at the most singular points of $\mathcal{X}_{0}$.

If, in addition, $\mathcal{X}$ is polarized with a choice of relatively ample line bundle $\mathcal{L}$, then the dual intersection complex $B$ comes equipped with a convex multi-valued piecewise linear function $\varphi$. This function can be used to define a discrete Legendre transform, which gives a new affine manifold $\check{B}$ which is, in a suitable sense, dual to $B$. In addition $\check{B}$ carries a new convex multi-valued piecewise linear function $\check{\varphi}$. Then $\check{B}$ should be the dual intersection complex of a mirror degeneration.

This part of the construction is relatively simple and conceptual, but it covers only the discrete part of mirror symmetry. It is the treatment of moduli that makes this paper so long. One apparent source of moduli is a change of gluing of the components of $\mathcal{X}_{0}$. However, an essential insight of this paper is that the correct limiting version of complex moduli also involves a choice of logarithmic structure. More justification for doing this will come from the study of deformation theory in the sequel of this paper. On the other hand, the source of the moduli on the Kähler side is perhaps less clear. In this paper we have fairly much reverse-engineered from the complex moduli side. The fact that the Kähler moduli space is then related (in an expected fashion) to the logarithmic Picard group should be seen as the first striking verification of our approach. In the sequel to this paper, we will connect the Kähler moduli more directly to a logarithmic version of $H^{1,1}$ and couple this with a base-change theorem, justifying our definition further [21].

Let us consider a simple example of the phenomena we have just outlined. Consider $\mathcal{X} \subseteq \mathbb{P}^{3} \times \mathbb{A}^{1}$ defined by the equation

$$
f_{4}+t x_{0} x_{1} x_{2} x_{3}=0
$$

for $f_{4}$ a general choice of homogeneous polynomial of degree four, with $t$ the coordinate on $\mathbb{A}^{1}$. Let $f: \mathcal{X} \rightarrow \mathbb{A}^{1}$ be the projection. Then $\mathcal{X}_{0}$ is a union of toric varieties $\left(\mathbb{P}^{2}\right.$ 's) meeting along toric strata, and the map is generically normal crossings, except at 24 points in $\mathcal{X}_{0}$ where the total space $\mathcal{X}$ is singular; call this set of points $Z$.

We can build the dual intersection complex $B$ of $\mathcal{X}$ as follows. Because $\mathcal{X}_{0}$ is normal crossings, $B$ will be a simplicial complex and coincide with the traditional dual intersection complex: we have a vertex for each 
irreducible component of $\mathcal{X}_{0}$, and if $v_{0}, \ldots, v_{k}$ are vertices corresponding to components $X_{v_{0}}, \ldots, X_{v_{k}}$, then $\left\langle v_{0}, \ldots, v_{k}\right\rangle$ is a simplex of the dual intersection complex if and only if $X_{v_{0}} \cap \cdots \cap X_{v_{k}} \neq \emptyset$. Thus in this case, $B$ is the boundary of a tetrahedron. $B$ carries a polyhedral decomposition $\mathscr{P}$, namely the collection of simplices of this simplicial complex. To make $B$ an affine manifold with singularities, we remove the midpoints of each edge; these will be singularities of the affine structure. Identify each face with the standard simplex in $\mathbb{R}^{2}$; this gives an affine structure in the interior of each face. Standard simplices appear here because $f: \mathcal{X} \backslash Z \rightarrow \mathbb{A}^{1}$ is normal crossings; in the more general case we will replace simplices by lattice polytopes.

To define affine charts in a neighbourhood of each vertex $v$, we specify a fan structure at $v$. This means we choose a complete rational polyhedral fan $\Sigma_{v}$ in $\mathbb{R}^{2}$, and a homeomorphism between an open neighbourhood of $v$ and an open neighbourhood of 0 in $\mathbb{R}^{2}$, giving a one-to-one correspondence between cells of $\mathscr{P}$ containing $v$ and cones in $\Sigma_{v}$, so that in the interior of each maximal cell of $\mathscr{P}$ containing $v$, the homeomorphism is an element of $\operatorname{Aff}\left(\mathbb{Z}^{2}\right)$. To construct the dual intersection complex, we take the fan $\Sigma_{v}$ to be the fan defining the irreducible component $X_{v}$. This gives the following picture:
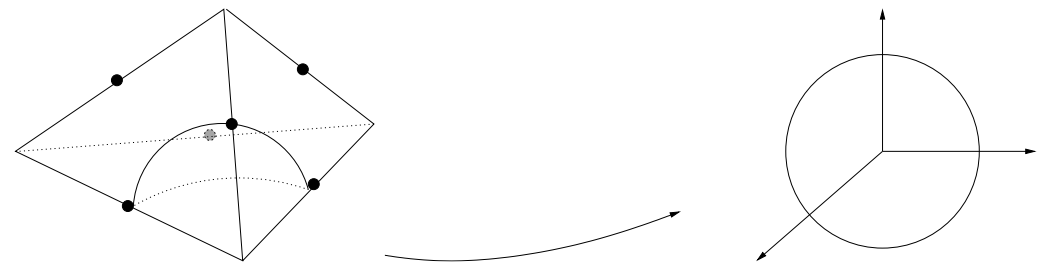

To construct the intersection complex $\check{B}$ of $\mathcal{X}$, we need to choose a polarization on $\mathcal{X}_{0}$, say given by the restriction of $\mathcal{O}_{\mathbb{P}^{3}}(n)$ for some $n$ to $\mathcal{X}_{0}$. Then restricting this line bundle to each irreducible component of $\mathcal{X}_{0}$, we take the Newton polytope of this line bundle, giving in our example the standard simplex of $\mathbb{R}^{2}$ rescaled by a factor of $n$. These are glued together in the obvious way to reflect the intersections of the components of $\mathcal{X}_{0}$, again yielding a tetrahedron in this example. This gives a polyhedral decomposition $\check{\mathscr{P}}$ on $\check{B}$ which is dual to $\mathscr{P}$. Finally we specify an affine structure with singularities by specifying a fan structure at each vertex. This time we take as fan the normal fan to the corresponding (maximal) cell of the dual intersection complex. The example of the quartic is perhaps misleading though because $\check{B}$ is the same thing as $B$, rescaled by a factor of $n$. We will see in $\S 4$, however, that it is always true that $B$ and $\check{B}$ are related by a discrete Legendre transform.

Now going from a toric degeneration to its intersection complex or dual intersection complex is easy; going backwards is more difficult, and 
we do not complete this task in this paper. Rather, we only show how to go from $(B, \mathscr{P})$ to a family of log Calabi-Yau spaces. To illustrate this, consider the case that $B$ is two dimensional, as above, and the polyhedral decomposition $\mathscr{P}$ subdivides $B$ into standard simplices. Then to each vertex $v \in \mathscr{P}$ is associated a fan $\Sigma_{v}$, obtained by looking at $\mathscr{P}$ in a small neighbourhood of $v$ in $B$. We then obtain a corresponding toric variety $X_{v}$. These varieties can then be glued together in a way whose combinatorics is specified by $B$ and $\mathscr{P}$ : we want to construct a scheme or algebraic space $X_{0}$ whose dual intersection complex is $(B, \mathscr{P})$. There is some moduli to this gluing, say specified by some data $s$. We then obtain spaces $X_{0}(B, \mathscr{P}, s)$ given by this gluing. In this simple case, $X_{0}(B, \mathscr{P}, s)$ is normal crossings, and as studied by Friedman in [12], the deformation theory of $X_{0}(B, \mathscr{P}, s)$ is controlled partly by a sheaf $\mathcal{N}_{D}=\mathcal{E} x t^{1}\left(\Omega_{X_{0}(B, \mathscr{P}, s)}^{1}, \mathcal{O}_{X_{0}(B, \mathscr{P}, s)}\right)$, a line bundle on the singular locus $D$ of $X_{0}(B, \mathscr{P}, s)$. It turns out this sheaf can be calculated in terms of $B$ and $s$, and in particular, the restriction of $\mathcal{N}_{D}$ to any irreducible component of $D$, corresponding to an edge $\tau$ of $\mathscr{P}$, is $\mathcal{O}_{\mathbb{P}^{1}}(n)$, where suitably defined monodromy of the affine structure is $\left(\begin{array}{cc}1 & n \\ 0 & 1\end{array}\right)$ around the singular point in the interior of $\tau$. (See Example 3.30. In the quartic example above, $n=4$ for each singular point.) In order for $X_{0}(B, \mathscr{P}, s)$ to be smoothable, $\mathcal{N}_{D}$ must have a section not generically zero along any component (and the zeroes will be related to the singular set $Z$ mentioned above). This places restrictions on both the numbers $n$ (obviously we need $n \geq 0$, which is the positivity condition of $\S 1.5$ ) and $s$. It turns out in this case specifying a section of $\mathcal{N}_{D}$ with no zeroes at singular points of $D$ is the same thing as choosing a log structure on $X_{0}(B, \mathscr{P}, s)$ and a morphism to a log point. Thus the full moduli space we are interested in is, in this example, the moduli space of pairs $X_{0}(B, \mathscr{P}, s)$ and sections of $\mathcal{N}_{D}$ not vanishing at any singular point of $D$. This can be viewed as a rough approximation to the moduli space of a smoothing of $X_{0}(B, \mathscr{P}, s)$ : they in fact have the same dimension.

For more general $B, \mathscr{P}$ or more general types of toric degenerations (not just normal crossings) the situation is much more complicated. This is the difficulty alluded to above which makes this paper so long. It is necessary to consider more general degenerations, for if we restricted to normal crossings degenerations, the mirror degenerations could only involve irreducible components isomorphic to $\mathbb{P}^{n}$.

As much more motivation was already given in [19], we will end this discussion here and rather give a summary and reader's guide to the paper. A lot of the content is quite technical, so a good deal can be skipped on a first reading, and we will try to explain what is or is not essential for absorbing the basic ideas of the paper. 
Section 1 is devoted entirely to affine manifolds, and the structures on them which will play a role in the remainder of the paper. Section 1.1 is intended as a warm-up, reminding the reader of basic concepts of affine manifolds, simple examples, and a review of the Legendre transform in this context. This provides important motivation, as the Legendre transform is key to Hitchin's elaboration of the SYZ (Strominger-YauZaslow) approach to mirror symmetry [48], [24]. As we replace the Legendre transform with the discrete Legendre transform, we are able to argue that our approach is an algebro-geometric version of SYZ.

Section 1.2 introduces affine manifolds with singularities, and gives some basic examples, such as Example 1.18. These first two sections are straightforward and are essential reading.

In $\S 1.3$, we introduce the basic combinatorial objects: polyhedral decompositions of integral affine manifolds with singularities. This is essentially just a decomposition of an integral affine manifold with singularities into lattice polytopes, though there is some subtlety in how such polytopes are allowed to interact with the singular set of the affine manifold. The definition, Definition 1.22, is essential for this paper, and Construction 1.26 is the prime example: we will use this to construct the discrete Legendre transform and the dual intersection complex. We note that both this definition and construction have already been given in [19]. The remainder of the section explores properties of polyhedral decompositions and auxiliary constructions. These are all quite important for the paper, but proofs may be skipped without great harm.

Section 1.4 describes the discrete Legendre transform and its properties: Proposition 1.50 and Proposition 1.51 summarize these. We make connections with the standard discrete Legendre transform on $\mathbb{R}^{n}$ in Example 1.52, and with Batyrev duality in Example 1.53.

Section 1.5 defines two properties of affine manifolds with singularities. The first is a positivity property, a generalisation of the phenomenon which occurs in elliptic fibrations which forces suitably normalized monodromy around Kodaira-type $I_{n}$ fibres to be positive in a certain sense. The second is simplicity, an analogue of the notion introduced for torus fibrations in [15]. These definitions, especially that of simplicity, are a bit technical, and the whole section could be skipped until these definitions are used in $\S \S 4$ and 5 .

In $\S 2$, we begin the process of constructing toric log Calabi-Yau spaces from pairs $(B, \mathscr{P})$, where $B$ is an integral affine manifold with singularities and $\mathscr{P}$ is a polyhedral decomposition on $B$. We actually give two dual constructions in $\S \S 2.1$ and 2.2 , depending on whether we view $(B, \mathscr{P})$ as the intersection complex or dual intersection complex of the degeneration. The former case, which we refer to as the cone picture, is technically easier and in fact produces a projective scheme (if $B$ is compact), whereas the latter case, which we refer to as the 
fan picture, is technically harder and produces only an algebraic space. In fact, most of $\S 2.2$ is devoted to constructing an explicit étale open cover of this algebraic space, which we will need for later aspects of the construction. This construction has a number of subtleties having to do with the role of the singularities in $B$. We recommend strongly reading $\S 2$ through Example 2.17, as this will illustrate these subtleties. Most of the rest of the section is devoted to the details of the construction of an algebraic space $X_{0}(B, \mathscr{P}, s)$ starting with data $(B, \mathscr{P})$ and so-called open gluing data $s$ (Definition 2.25). This can be skimmed, but open gluing data will play an important role. At the end of $\S 2.2$, we compare the fan and cone pictures, and compute the dualizing sheaf and basic invariants of $X_{0}(B, \mathscr{P}, s)$. These statements should be read.

Section 3 introduces log structures. The main new idea in this paper is the significance of $\log$ structures in mirror symmetry. While the definition of a log structure is very simple, learning to work with them can take some time. So $\S 3.1$ provides an introduction to the sorts of log structures we use. Given a toric degeneration $f: \mathcal{X} \rightarrow \mathcal{S}$, the special fibre $\mathcal{X}_{0}$ inherits a canonical log structure from the inclusion $\mathcal{X}_{0} \subseteq \mathcal{X}$. This log structure should be thought of as providing key information about the smoothing. Furthermore, while our construction of the dual intersection complex as an affine manifold depends not just on $\mathcal{X}_{0}$ but the map $f: \mathcal{X} \rightarrow \mathcal{S}$, in fact all the information needed about $f$ is contained in the $\log$ structure on $\mathcal{X}_{0}$. So our dual intersection complex construction makes sense not just for toric degenerations, but also for what we call toric log Calabi-Yau spaces, log spaces which look like degenerate fibres of toric degenerations, defined over the standard log point. Thus, if one wants to understand mirror symmetry by studying singular fibres of degenerations, one cannot work just with the singular fibres, but must also involve the $\log$ structure. In fact, the discrete Legendre transform will interchange information about the irreducible components of a toric log Calabi-Yau space with information about the log structure of its mirror. Hence, log structures lie at the heart of our construction. Section 3.1 is thus essential reading.

In $\S \S 3.2$ and 3.3 we begin to address the question: given an affine manifold with singularities $B$ with polyhedral decomposition $\mathscr{P}$ and open gluing data $s$, how do we put a log structure on $X_{0}(B, \mathscr{P}, s)$ in order to make it a log Calabi-Yau space? We are able to construct a sheaf of sets on $X_{0}(B, \mathscr{P}, s)$ whose sections define suitable log structures. This construction is explained in $\S 3.2$, and in $\S 3.3$, we actually compute this sheaf explicitly. This latter section is the technical heart of the paper, and can probably be skipped on a first reading. The eventual goal is to identify global sections of this sheaf; this is finally accomplished in the case that $B$ is positive and simple in Theorem 5.2, with one of the main theorems of the paper being Theorem 5.4. This allows us, in the 
simple case, to calculate the moduli space of log Calabi-Yau spaces with a given dual intersection complex $(B, \mathscr{P})$. This moduli space will play the role of complex moduli in mirror symmetry for toric log Calabi-Yau spaces.

Section 4 introduces the notions of toric degenerations and log CalabiYau spaces, and in $\S 4.1$ we give these definitions and the construction of the dual intersection complex, reversing the constructions of the previous two chapters. The construction of the dual intersection complex was already explained in less detail in [19]. A brief $\S 4.2$ discusses the polarized case and the intersection complex. Section 4.3 makes the connection with positivity: we show the dual intersection complex of a toric degeneration of Calabi-Yau varieties is always positive, hence justifying the definition of positive. Finally, in $\S 4.4$, we continue some of the calculations leading up to the proof of Theorems 5.2 and 5.4. This is again technical, but Examples 4.28 and 4.29 should prove informative.

Section 5 ties together all the strands so far. We complete the calculation of the moduli of log Calabi-Yau spaces with a given dual intersection complex in the simple case. The proof relies on most of the technical aspects developed so far in the paper. However, the answer is elegant: the moduli space in fact coincides with a cohomology group of a natural sheaf on $B$, determined canonically by the affine structure. Furthermore, this is precisely the group expected from previous experience with the Strominger-Yau-Zaslow conjecture. In $\S 5.2$, we compute the log Picard group; this can probably be skipped altogether as it merely provides motivation for the definition of the log Kähler moduli space in §5.3. The log Kähler moduli space will be canonically isomorphic to the moduli space of log Calabi-Yau spaces for the mirror. There is no technical content in $\S 5.3$, and in fact the reader may wish to read this section early on! There we also describe the many aspects of this program left undone.

Remark 0.1. Contrary to $[\mathbf{3 8}]$ or $[\mathbf{1 3}]$, we use the following convention. Let $\Sigma$ be a fan defining a toric variety $X$, with toric Weil divisors $D_{1}, \ldots, D_{n}$ corresponding to rays $R_{1}, \ldots, R_{n}$ of $\Sigma$ with primitive generators $v_{1}, \ldots, v_{n}$. If $D=\sum a_{i} D_{i}$ is a Cartier divisor, we take the piecewise linear function $\psi$ on the fan $\Sigma$ corresponding to $D$ to take the values $\psi\left(v_{i}\right)=a_{i}$, rather than $\psi\left(v_{i}\right)=-a_{i}$, as in [38] or [13]. This will affect various signs throughout.

Acknowledgements. We would like to thank Klaus Altmann, Robert Friedman, Maxim Kontsevich, Marco Kühnel, Arthur Ogus, Martin Olsson, Simone Pavanelli, Stefan Schröer, Balazs Szendröi, Richard Thomas, and Ilia Zharkov for useful conversations during the progress of this work.

Part of this work has been done while the first author visited RIMS in Kyoto and the second author stayed at the Institut de Mathématiques 
de Jussieu in Paris. They thank these named institutions for hospitality, and in particular their respective hosts, Kyoji Saito and Claire Voisin. We would also especially like to thank the referee for his invaluable comments.

\section{Affine Manifolds}

1.1. Affine Manifolds and Invariants. We will start by reviewing some basic notions concerning affine manifolds and their relation to mirror symmetry. For basic information on affine manifolds, we follow Goldman and Hirsch's paper [14].

We fix $M=\mathbb{Z}^{n}$ an abelian group, $N=\operatorname{Hom}_{\mathbb{Z}}(M, \mathbb{Z}), M_{\mathbb{R}}=M \otimes_{\mathbb{Z}} \mathbb{R}$, $N_{\mathbb{R}}=N \otimes_{\mathbb{Z}} \mathbb{R}$. Then $N=M^{*}$ and $N_{\mathbb{R}}=M_{\mathbb{R}}^{*}$ if $\Lambda^{*}=\operatorname{Hom}_{\mathbb{Z}}(\Lambda, \mathbb{Z})$ and $V^{*}=\operatorname{Hom}_{\mathbb{R}}(V, \mathbb{R})$ for an abelian group $\Lambda$ and an $\mathbb{R}$-vector space $V$ respectively, and $\otimes_{\mathbb{Z}} \mathbb{R}$ is viewed as functor taking abelian groups to $\mathbb{R}$-vector spaces.

We set

$$
\operatorname{Aff}\left(M_{\mathbb{R}}\right)=M_{\mathbb{R}} \rtimes \mathrm{GL}_{n}(\mathbb{R})
$$

to be the group of affine transformations of $M_{\mathbb{R}}$, with subgroup

$$
\operatorname{Aff}(M)=M \rtimes \mathrm{GL}_{n}(\mathbb{Z}) .
$$

If $M$ and $M^{\prime}$ are two different lattices, then we denote by $\operatorname{Aff}\left(M_{\mathbb{R}}, M_{\mathbb{R}}^{\prime}\right)$ the $\mathbb{R}$-vector space of affine maps between $M_{\mathbb{R}}$ and $M_{\mathbb{R}}^{\prime}$. Here

$$
\operatorname{Aff}\left(M_{\mathbb{R}}, M_{\mathbb{R}}^{\prime}\right)=M_{\mathbb{R}}^{\prime} \times \operatorname{Hom}\left(M_{\mathbb{R}}, M_{\mathbb{R}}^{\prime}\right) .
$$

Similarly

$$
\operatorname{Aff}\left(M, M^{\prime}\right)=M^{\prime} \times \operatorname{Hom}\left(M, M^{\prime}\right)
$$

is the $\mathbb{Z}$-module of affine maps between $M$ and $M^{\prime}$.

Definition 1.1. Let $B$ be an $n$-dimensional manifold. An affine structure on $B$ is given by an open cover $\left\{U_{i}\right\}$ along with coordinate charts $\psi_{i}: U_{i} \rightarrow M_{\mathbb{R}}$, whose transition functions $\psi_{i} \circ \psi_{j}^{-1}$ lie in $\operatorname{Aff}\left(M_{\mathbb{R}}\right)$. The affine structure is integral if the transition functions lie in $\operatorname{Aff}(M)$. If $B$ and $B^{\prime}$ are (integral) affine manifolds of dimensions $n$ and $n^{\prime}$ respectively, then a continuous map $f: B \rightarrow B^{\prime}$ is (integral) affine if locally $f$ is given by elements of $\operatorname{Aff}\left(\mathbb{R}^{n}, \mathbb{R}^{n^{\prime}}\right)\left(\operatorname{Aff}\left(\mathbb{Z}^{n}, \mathbb{Z}^{n^{\prime}}\right)\right)$. If in addition $f$ is a local diffeomorphism, we say $f$ is étale (integral) affine.

Remark 1.2. In other papers on the role of affine manifolds in mirror symmetry [31], [23], affine manifolds with transition maps in $M_{\mathbb{R}} \rtimes \mathrm{GL}_{n}(\mathbb{Z})$ are considered. We restrict to the integral case here because these are the affine manifolds which arise when studying degenerations; this is roughly equivalent on the mirror side to focusing on symplectic manifolds with integral symplectic forms. Dual intersection complexes arising from toric degenerations are integral affine manifolds with singularities, as are intersection complexes arising from a choice of 
ample line bundle on a toric degeneration. In particular, the examples given in the introduction associated to the quartic are integral.

Proposition 1.3. Let $\pi: \tilde{B} \rightarrow B$ be the universal covering of an (integral) affine manifold $B$, inducing an (integral) affine structure on $\tilde{B}$. Then there is an étale (integral) affine map $\delta: \tilde{B} \rightarrow M_{\mathbb{R}}$, called the developing map, and any two such maps differ only by an (integral) affine transformation.

Proof. This is standard, see [14], p. 641 for a proof.

q.e.d.

Note that there is no need for the developing map to be injective or a covering space; it is only a local isomorphism in general.

Definition 1.4. The fundamental group $\pi_{1}(B)$ acts on $\tilde{B}$ by deck transformations; for $\gamma \in \pi_{1}(B)$, let $\Psi_{\gamma}: \tilde{B} \rightarrow \tilde{B}$ be the corresponding deck transformation with $\Psi_{\gamma_{2}} \circ \Psi_{\gamma_{1}}=\Psi_{\gamma_{1} \gamma_{2}}$. Then by the uniqueness of the developing map, there exists a $\rho(\gamma) \in \operatorname{Aff}\left(M_{\mathbb{R}}\right)$ such that $\rho(\gamma) \circ$ $\delta \circ \Psi_{\gamma}=\delta$. The map $\rho: \pi_{1}(B) \rightarrow \operatorname{Aff}\left(M_{\mathbb{R}}\right)$ is called the holonomy representation. If the affine structure is integral, then $\operatorname{im} \rho \subseteq \operatorname{Aff}(M)$.

Recall that we compose loops $\gamma_{1}$ and $\gamma_{2}$ in $\pi_{1}(B)$ so that $\gamma_{1} \gamma_{2}$ is obtained by first following $\gamma_{1}$ and then $\gamma_{2}$. Hence $\Psi_{\gamma_{1} \gamma_{2}}=\Psi_{\gamma_{2}} \circ \Psi_{\gamma_{1}}$, from which it follows we have defined $\rho$ to be a group homomorphism. A different way to view this is by observing that $B$ is naturally endowed with an affine connection (as opposed to the ubiquitous linear connections) by pulling back the standard affine connection on $M_{\mathbb{R}} \cong \mathbb{R}^{n}$ via charts. Then $\rho(\gamma)$ is given by parallel transport along $\gamma^{-1}$ with respect to the affine connection. (See $[\mathbf{1 4}]$.)

Note conversely that given an immersion $\delta: \tilde{B} \rightarrow M_{\mathbb{R}}$ and a representation $\rho: \pi_{1}(B) \rightarrow \operatorname{Aff}\left(M_{\mathbb{R}}\right)$ such that $\rho(\gamma) \circ \delta \circ \Psi_{\gamma}=\delta$, these data induce an affine structure on $B$.

\section{Example 1.5.}

1) If $B$ is an (integral) affine manifold and $G$ is a group acting properly and discontinuously on $B$ via (integral) affine transformations, then $B / G$ inherits an (integral) affine structure from $B$.

2) As $M_{\mathbb{R}}$ is naturally an affine manifold, with the developing map being the identity, if $\Gamma \subseteq M_{\mathbb{R}}$ is any lattice acting by translations on $M_{\mathbb{R}}$, we obtain an affine structure on $M_{\mathbb{R}} / \Gamma$. Here for $\lambda \in$ $\pi_{1}\left(M_{\mathbb{R}} / \Gamma\right)=\Gamma, \rho(\lambda)$ is a translation by $-\lambda$. Thus the affine structure is integral if and only if $\Gamma \subseteq M$.

3) This example is from [5]. Take $M=\mathbb{Z}^{2}$, and consider the subgroup $G \subseteq \operatorname{Aff}\left(M_{\mathbb{R}}\right)$ consisting of all $A \in \operatorname{Aff}(M, \mathbb{R})$ of the form

$$
A_{u, v}\left(m_{1}, m_{2}\right)=\left(m_{1}+v m_{2}+u+v(v-1) / 2, m_{2}+v\right),
$$

for $u, v \in \mathbb{R}$. (This is not quite the form given in [5], but rather $G$ has been conjugated by translation by $(0,-1 / 2)$ to obtain better 
integrality properties). $G$ is isomorphic to $\mathbb{R}^{2}$, and if we choose any lattice $\Gamma \subseteq G$, then $\Gamma$ acts properly and discontinuously, so that $M_{\mathbb{R}} / \Gamma$ is an affine manifold, topologically a two-torus. This is the only other affine structure on the two-torus obtained from $M_{\mathbb{R}}$ by dividing out by a properly discontinuous group action. (See [5], Theorem 4.5). Note the affine structure is integral with respect to the integral structure $M \subseteq M_{\mathbb{R}}$ if and only if

$$
\Gamma \subseteq\left\{A_{u, v} \in \operatorname{Aff}\left(M_{\mathbb{R}}\right) \mid u, v \in \mathbb{Z}\right\} .
$$

We recall the notion of radiance obstruction from [14].

Definition 1.6. Let Lin : $\operatorname{Aff}\left(M_{\mathbb{R}}\right) \rightarrow \mathrm{GL}_{n}(\mathbb{R})$ and Trans : $\operatorname{Aff}\left(M_{\mathbb{R}}\right)$ $\rightarrow M_{\mathbb{R}}$ be the projections. Here Lin is a homomorphism, but Trans is a crossed homomorphism with respect to the regular representation of $\mathrm{GL}_{n}(\mathbb{R})$ on $M_{\mathbb{R}}$, i.e.,

$$
\operatorname{Trans}\left(A_{1} A_{2}\right)=\operatorname{Lin}\left(A_{1}\right)\left(\operatorname{Trans}\left(A_{2}\right)\right)+\operatorname{Trans}\left(A_{1}\right) .
$$

Given an affine representation $\rho: G \rightarrow \operatorname{Aff}\left(M_{\mathbb{R}}\right)$ of a group $G$, set $\tilde{\rho}=$ Lin $\circ \rho$. Then Trans $\circ \rho$ can be interpreted as an element $c_{\rho} \in H^{1}\left(G, M_{\mathbb{R}}^{\tilde{\rho}}\right)$, where $M_{\mathbb{R}}^{\tilde{\rho}}$ denotes the $G$-module $M_{\mathbb{R}}$ defined by the representation $\tilde{\rho}$. The class $c_{\rho}$ is called the radiance obstruction of $\rho$. If $B$ is an affine manifold and $\rho: \pi_{1}(B) \rightarrow \operatorname{Aff}\left(M_{\mathbb{R}}\right)$ is the holonomy representation of $B$, then the radiance obstruction of $B$ is $c_{B_{0}}:=c_{\rho}$.

The radiance obstruction is an important invariant of an affine manifold. It can be viewed as being analogous to the cohomology class of the symplectic form on a symplectic manifold.

Theorem 1.7. Two affine representations $\rho_{1}, \rho_{2}: G \rightarrow \operatorname{Aff}\left(M_{\mathbb{R}}\right)$ with $\tilde{\rho}_{1}=\tilde{\rho}_{2}$ are conjugate by a translation if and only if $c_{\rho_{1}}=c_{\rho_{2}}$.

Proof. [14], p. 631.

q.e.d.

This is important for the following reason. If we compose an affine structure with a translation, i.e., replace $\delta$ with $\tau_{a} \circ \delta$ where $\tau_{a}$ denotes translation by $a$, then the holonomy representation $\rho$ is replaced by $\rho^{\prime}$ with $\rho^{\prime}(\gamma)=\tau_{a} \circ \rho(\gamma) \circ \tau_{a}^{-1}$, and then Trans $\circ \rho$ and Trans $\circ \rho^{\prime}$ are cohomologous:

$$
\operatorname{Trans}\left(\rho^{\prime}(\gamma)\right)=a-\tilde{\rho}(\gamma)(a)+\operatorname{Trans}(\rho(\gamma)) .
$$

Thus holonomy representations from affine structures related by translation are conjugate by a translation, so the radiance obstruction helps classify holonomy representations. In particular, this allows us to identify integral affine structures:

Proposition 1.8. An affine representation $\rho: G \rightarrow M_{\mathbb{R}} \rtimes \mathrm{GL}_{n}(\mathbb{Z}) \subseteq$ $\operatorname{Aff}\left(M_{\mathbb{R}}\right)$ is conjugate by a translation to a representation $\rho^{\prime}: G \rightarrow$ 
$\operatorname{Aff}(M)$ if and only if the radiance obstruction $c_{\rho} \in H^{1}\left(G, M_{\mathbb{R}}^{\tilde{\rho}}\right)$ is in the image of $H^{1}\left(G, M^{\tilde{\rho}}\right) \rightarrow H^{1}\left(G, M_{\mathbb{R}}^{\tilde{\rho}}\right)$.

Proof. If $\rho$ is conjugate to a representation $\rho^{\prime}: G \rightarrow \operatorname{Aff}(M)$ then $u=$ Trans $\circ \rho^{\prime}: G \rightarrow M$ is a crossed homomorphism representing an element of $H^{1}\left(G, M^{\tilde{\rho}}\right)$ whose image in $H^{1}\left(G, M_{\mathbb{R}}^{\tilde{\rho}}\right)$ is $c_{\rho}$. Conversely, if $c_{\rho}$ is in the image of $H^{1}\left(G, M^{\tilde{\rho}}\right)$, then $u$ is cohomologous to a $v: G \rightarrow M^{\tilde{\rho}}$, i.e., there exists $a \in M_{\mathbb{R}}$ such that $u(g)-v(g)=a-\tilde{\rho}(g) a$. If $\tau_{a}$ denotes translation by $a$, then this says $\tau_{a}^{-1} \circ \rho(g) \circ \tau_{a}$ is in $\operatorname{Aff}(M)$ for all $g \in G$.

q.e.d.

The radiance obstruction in fact also plays a role in understanding the Legendre transform, as we shall see, and this is the primary reason for introducing it here.

Definition 1.9. If $B$ is an affine manifold, there is a flat linear connection $\nabla$ on $\mathcal{T}_{B}$, where if $y_{1}, \ldots, y_{n}$ are local affine coordinates, $\partial / \partial y_{1}, \ldots, \partial / \partial y_{n}$ are a frame of flat sections of $\mathcal{T}_{B}$. Denote by $\Lambda_{\mathbb{R}}$ the local system of flat sections and $\check{\Lambda}_{\mathbb{R}}$ the dual local system of flat sections of the dual connection on $\mathcal{T}_{B}$. If furthermore the holonomy of $B$ is contained in $M_{\mathbb{R}} \rtimes \mathrm{GL}_{n}(\mathbb{Z})$, rather than $M_{\mathbb{R}} \rtimes \mathrm{GL}_{n}(\mathbb{R})$, then there exist integral subsystems $\Lambda \subseteq \Lambda_{\mathbb{R}}$ and $\check{\Lambda} \subseteq \check{\Lambda}_{\mathbb{R}}$ coming from the inclusions $M \subseteq M_{\mathbb{R}}$ and $N \subseteq N_{\mathbb{R}}$.

We note that the monodromy of the local system $\Lambda_{\mathbb{R}}$ is precisely the linear part of the holonomy representation. Thus the radiance obstruction can be viewed as measuring the difference between the monodromy of $\Lambda_{\mathbb{R}}$ and the holonomy representation.

Remark 1.10. [14] gives a number of ways of realising the radiance obstruction. The Čech realisation will also be of use to us.

Choose an open covering $\left\{U_{i}\right\}$ of $B$ along with affine charts $\psi_{i}$ : $U_{i} \rightarrow M_{\mathbb{R}}$. Such a chart allows us to identify $\mathcal{T}_{U_{i}}$ canonically with $U_{i} \times M_{\mathbb{R}}$ and the graph of $\psi_{i}$ can be viewed as a section $s_{i} \in \Gamma\left(U_{i}, \mathcal{T}_{U_{i}}\right)$, which is parallel for the affine connection, and hence is independent of $\psi_{i}$ up to addition by flat sections of $\mathcal{T}_{U_{i}}$. Then $\left(s_{j}-s_{i}\right)_{i j}$ forms a Čech 1-cocycle for $\Lambda_{\mathbb{R}}$, and hence represents an element of $H^{1}\left(B, \Lambda_{\mathbb{R}}\right)$. This group is naturally isomorphic to $H^{1}\left(\pi_{1}(B), M_{\mathbb{R}}^{\tilde{\rho}}\right)$, and this Čech 1-cocycle represents the radiance obstruction under this isomorphism. If the charts $\psi_{i}$ are integral, then $s_{j}-s_{i} \in \Gamma\left(U_{i} \cap U_{j}, \Lambda\right)$, yielding the radiance obstruction in $H^{1}(B, \Lambda)$.

Definition 1.11. Let $\mathcal{A f f}_{\mathbb{R}}(B, \mathbb{R})$ denote the sheaf of affine maps from $B$ to $\mathbb{R}$, i.e., functions locally of the form $f \circ \delta$, where $f \in$ $\operatorname{Aff}\left(M_{\mathbb{R}}, \mathbb{R}\right)$ and $\delta$ is the developing map. This sheaf fits into a natural exact sequence

$$
0 \longrightarrow \mathbb{R} \longrightarrow \mathcal{A} f_{\mathbb{R}}(B, \mathbb{R}) \longrightarrow \check{\Lambda}_{\mathbb{R}} \longrightarrow 0,
$$


MIRROR SYMMETRY VIA LOGARITHMIC DEGENERATION DATA I 181

analogous to the exact sequence of $\mathbb{R}$-vector spaces

$$
0 \longrightarrow \mathbb{R} \longrightarrow \operatorname{Aff}\left(M_{\mathbb{R}}, \mathbb{R}\right) \longrightarrow N_{\mathbb{R}} \longrightarrow 0 .
$$

Similarly, if $B$ is an integral affine manifold, define $\mathcal{A f f}(B, \mathbb{Z})$ to be the sheaf of affine functions on $B$ locally of the form $f \circ \delta$, where $f \in$ $\operatorname{Aff}(M, \mathbb{Z})$. There is an exact sequence

$$
0 \longrightarrow \mathbb{Z} \longrightarrow \mathcal{A} f(B, \mathbb{Z}) \longrightarrow \check{\Lambda} \longrightarrow 0 \text {. }
$$

Another description of the radiance obstruction:

Proposition 1.12. The extension class of

$$
0 \longrightarrow \mathbb{R} \longrightarrow \mathcal{A f f} f_{\mathbb{R}}(B, \mathbb{R}) \longrightarrow \check{\Lambda}_{\mathbb{R}} \longrightarrow 0
$$

in $\operatorname{Ext}^{1}\left(\check{\Lambda}_{\mathbb{R}}, \mathbb{R}\right)=H^{1}\left(B, \Lambda_{\mathbb{R}}\right)$ coincides with the radiance obstruction of $B$.

Proof. Let $\left\{U_{i}\right\}$ be a cover of $B$ of contractible open sets, and on each $U_{i}$ choose a splitting $\alpha_{i}: \check{\Lambda}_{\mathbb{R}} \rightarrow \mathcal{A} f f_{\mathbb{R}}(B, \mathbb{R})$. Then the extension class is determined by the Cech cocycle $\left(\alpha_{j}-\alpha_{i}\right)_{i j}, \alpha_{j}-\alpha_{i}: \check{\Lambda}_{\mathbb{R}} \rightarrow \mathbb{R}$. To compare this with the radiance obstruction, note $\alpha_{i}$ determines an affine chart $\psi_{i}: U_{i} \rightarrow \Gamma\left(U_{i}, \Lambda_{\mathbb{R}}\right)=\operatorname{Hom}\left(\Gamma\left(U_{i}, \check{\Lambda}_{\mathbb{R}}\right), \mathbb{R}\right)$ by $\psi_{i}(b)(n)=\alpha_{i}(n)(b)$, for $b \in U_{i}, n \in \Gamma\left(U_{i}, \check{\Lambda}_{\mathbb{R}}\right)$ so that $\alpha_{i}(n)$ is an affine linear function on $U_{i}$. As in Remark 1.10, each chart $\psi_{i}$ determines a well-defined section $s_{i} \in \Gamma\left(U_{i}, \mathcal{T}_{U_{i}}\right)$, the graph of $\psi_{i}$, and the radiance obstruction is represented by $\left(s_{j}-s_{i}\right)_{i j}$. But for $b \in U_{i} \cap U_{j},\left(s_{j}-s_{i}\right)(b)$ is the functional on $\check{\Lambda}_{\mathbb{R}, b}=\mathcal{T}_{B, b}^{*}$ given by $\left(s_{j}-s_{i}\right)(b)(n)=\alpha_{j}(n)(b)-\alpha_{i}(n)(b)$. But this is precisely $\alpha_{j}-\alpha_{i}: \check{\Lambda}_{\mathbb{R}, b} \rightarrow \mathbb{R}$, which doesn't depend on $b$, so $s_{j}-s_{i}$ and $\alpha_{j}-\alpha_{i}$ coincide.

q.e.d.

To make contact with the metric form of SYZ, we briefly discuss metrics on affine manifolds.

Definition 1.13. Let $B$ be an affine manifold. A Hessian metric $g$ on $B$ is a Riemannian metric on $B$ such that locally, for affine coordinates $\left(y_{1}, \ldots, y_{n}\right)$, there is a potential function $K$ such that $g_{i j}=\partial^{2} K / \partial y_{i} \partial y_{j}$. The pair $(B, g)$ is called an affine Kähler manifold or a Hessian manifold. This can be defined in a coordinate independent way as $g=\nabla d K$, a section of the bundle $S^{2} \mathcal{T}^{*} \subseteq \mathcal{T}^{*} \otimes \mathcal{T}^{*}$.

Affine Kähler metrics were first studied by Cheng and Yau in [9], where metrics whose potential in addition satisfies the Monge-Ampère equation were studied. Such metrics should be especially important in the study of mirror symmetry, but we do not pursue this further here. Affine Kähler manifolds were called Hessian manifolds in [46].

The potential function $K$ is only defined locally, up to an affine function. Thus $K$ can actually be defined as a multi-valued function on 
$B$ well defined up to affine functions, and we consider such functions more generally.

Let $B$ be an affine manifold, $\pi: \tilde{B} \rightarrow B$ the universal cover and $\delta$ : $\tilde{B} \rightarrow M_{\mathbb{R}}$ the developing map. We consider continuous maps $\varphi: \tilde{B} \rightarrow \mathbb{R}$ which satisfy the condition

$$
\varphi-\varphi \circ \Psi_{\gamma}=\alpha(\gamma)
$$

where $\alpha$ is a map $\alpha: \pi_{1}(B) \rightarrow \operatorname{Aff}\left(M_{\mathbb{R}}, \mathbb{R}\right)$. Here an element of $\operatorname{Aff}\left(M_{\mathbb{R}}, \mathbb{R}\right)$ induces a map on $\tilde{B}$ via composition with $\delta$. Of course $\operatorname{Aff}\left(M_{\mathbb{R}}, \mathbb{R}\right)$ is a left $\pi_{1}(B)$-module, with $\gamma$ acting by composition on the right with $\rho\left(\gamma^{-1}\right)$ (or thinking of an element of $\operatorname{Aff}\left(M_{\mathbb{R}}, \mathbb{R}\right)$ as a map on $\tilde{B} \rightarrow \mathbb{R}, \gamma$ acts by composition with $\Psi_{\gamma}$ ). Then $\alpha$ is a crossed homomorphism:

$$
\begin{aligned}
\alpha\left(\gamma_{1} \gamma_{2}\right) & =\varphi-\varphi \circ \Psi_{\gamma_{2}} \circ \Psi_{\gamma_{1}} \\
& =\varphi-\left(\varphi-\alpha\left(\gamma_{2}\right)\right) \circ \Psi_{\gamma_{1}} \\
& =\gamma_{1}\left(\alpha\left(\gamma_{2}\right)\right)+\alpha\left(\gamma_{1}\right) .
\end{aligned}
$$

Thus $\alpha$ defines an element $[\alpha] \in H^{1}\left(\pi_{1}(B), \operatorname{Aff}\left(M_{\mathbb{R}}, \mathbb{R}\right)\right)$. Furthermore, adding an element of $\operatorname{Aff}\left(M_{\mathbb{R}}, \mathbb{R}\right)$ to $\varphi$ replaces $\alpha$ with a cohomologous $\alpha^{\prime}$, and any representative of $[\alpha]$ can be obtained in this way.

Alternatively, we can define $[\alpha] \in H^{1}\left(B, \mathcal{A f f}_{\mathbb{R}}(B, \mathbb{R})\right) \cong H^{1}\left(\pi_{1}(B)\right.$, $\left.\operatorname{Aff}\left(M_{\mathbb{R}}, \mathbb{R}\right)\right)$. Choosing a covering of $B$ by simply connected sets $U_{i}$ and choosing a representative $\varphi_{i}$ for $\varphi$ on $U_{i}, \varphi_{i}-\varphi_{j} \in \mathcal{A} f f_{\mathbb{R}}\left(U_{i} \cap U_{j}, \mathbb{R}\right)$ and hence we obtain a Čech cocycle in $H^{1}\left(B, \mathcal{A} f f_{\mathbb{R}}(B, \mathbb{R})\right)$.

Suppose now that $B$ carries a metric of Hessian form. Any two local potential functions $K$ for the metric differ by an affine function, so we can patch to get $K: \tilde{B} \rightarrow \mathbb{R}$. If $K-K \circ \Psi_{\gamma}=\alpha(\gamma)$, the class $[\alpha] \in H^{1}\left(\pi_{1}(B), \operatorname{Aff}\left(M_{\mathbb{R}}, \mathbb{R}\right)\right) \cong H^{1}\left(B, \mathcal{A} f f_{\mathbb{R}}(B, \mathbb{R})\right)$ is called the class of the metric $g$, as defined by Kontsevich and Soibelman [31].

The developing map yields an isomorphism

$$
\delta^{*}: \delta^{*} \mathcal{T}_{M_{\mathbb{R}}}^{*} \rightarrow \mathcal{T}_{\tilde{B}}^{*}
$$

Since $\mathcal{T}_{M_{\mathbb{R}}}^{*}=M_{\mathbb{R}} \times N_{\mathbb{R}}, \delta^{*}$ gives an isomorphism of $\tilde{B} \times N_{\mathbb{R}}$ with $\mathcal{T}_{\tilde{B}}^{*}$. Let $q: \tilde{B} \times N_{\mathbb{R}} \rightarrow N_{\mathbb{R}}$ be the projection. Then $\left(\delta^{*}\right)^{-1}(d K)$ is a section of $\delta^{*} \mathcal{T}_{M_{\mathbb{R}}}^{*}$, so we can view

$$
\check{\delta}:=q \circ\left(\delta^{*}\right)^{-1}(d K)
$$

as a function $\check{\delta}: \tilde{B} \rightarrow N_{\mathbb{R}}$. This is just the differential $d K$ under these identifications. Because the Hessian of $K$ is positive definite, $\check{\delta}$ is an immersion, hence defining a new affine structure on $\tilde{B}$. Note that $d \alpha(\gamma)$ is naturally identified with an element of $N_{\mathbb{R}}$. Under this identification, one can check that

$$
{ }^{t} \tilde{\rho}\left(\gamma^{-1}\right) \circ \check{\delta} \circ \Psi_{\gamma}+d \alpha(\gamma)=\check{\delta}
$$


and thus $\pi_{1}(B)$ acts on the new affine structure on $\tilde{B}$ by affine transformations. Dividing $\tilde{B}$ by this action, we obtain a new affine structure on $B$, which we denote by $\check{B}$. So the holonomy representation $\check{\rho}: \pi_{1}(\check{B}) \rightarrow \operatorname{Aff}\left(N_{\mathbb{R}}\right)$ of the affine structure given by $\check{\delta}$ has linear part $\tilde{\rho}^{*}$ dual to the representation $\tilde{\rho}$. Also $d \alpha(\gamma) \in N_{\mathbb{R}}$ is just the projection of $\alpha(\gamma) \in \operatorname{Aff}\left(M_{\mathbb{R}}, \mathbb{R}\right)$ onto $N_{\mathbb{R}}$. Thus the radiance obstruction of the affine structure $\check{\delta}$ is just the projection of $[\alpha] \in H^{1}\left(\pi_{1}(\check{B}), \operatorname{Aff}\left(M_{\mathbb{R}}, \mathbb{R}\right)\right)$ to $H^{1}\left(\pi_{1}(B), N_{\mathbb{R}}\right)$.

We can also define the Legendre transform of $K$ as, for $x \in \tilde{B}$,

$$
\check{K}(x)=\langle\check{\delta}(x), \delta(x)\rangle-K(x) .
$$

Then the Hessian of $\check{K}$ defines the same metric as $K$ on $B$, and $d \check{K}=\delta$. For a proof of this, see [24], $\S 5$. We call $(\check{B}, \check{K})$ the Legendre transform of $(B, K)$. We note we have shown the radiance obstruction of $\check{B}$ is determined by the class of the metric. Conversely, the class of the metric on $\check{B}$ is at least partially determined by the radiance obstruction of $B$, since $d \check{K}=\delta$.

The role of the Legendre transform in the SYZ picture of mirror symmetry is well-understood, and was first explained by Hitchin [24]. See also [32], which develops this point of view further. It is precisely the presence of the potential function $K$ which allows us to pass between $B$ and $\check{B}$, thus dualizing affine manifolds. One of the key points of this paper is that we can in fact formulate a discrete version of this, and replace $K$ with a piecewise linear function. This will enable us to obtain an algebro-geometric analog of SYZ.

Example 1.14. In Example 1.5, (2), we can take any convex quadratic function $K: M_{\mathbb{R}} \rightarrow \mathbb{R}$ to serve as a potential. Now $K$ satisfies the periodicity condition

$$
K(x+\gamma)=K(x)+\alpha(\gamma)(x)
$$

for $\gamma \in \Gamma, x \in M_{\mathbb{R}}$, and some $\alpha(\gamma) \in \operatorname{Aff}\left(M_{\mathbb{R}}, \mathbb{R}\right)$. Taking differentials of this equation, we get $\check{\delta}(x+\gamma)=\check{\delta}(x)+d \alpha(\gamma) \in N_{\mathbb{R}}$. Thus if we set

$$
\check{\Gamma}=\left\{d \alpha(\gamma) \in N_{\mathbb{R}} \mid \gamma \in \Gamma\right\},
$$

then the dual torus can be identified as $N_{\mathbb{R}} / \check{\Gamma}$.

In Example 1.5, (3), in fact there is no convex function $K: M_{\mathbb{R}} \rightarrow \mathbb{R}$ defining a Hessian metric on $B$. (The easiest way to see this is to note that the torus bundle $X(B):=\mathcal{T}_{B} / \Lambda$ has a natural complex structure on it making $X(B)$ isomorphic to a primary Kodaira surface, and the pull-back of $K$ to $X(B)$ is the Kähler potential of a Kähler metric. However, a primary Kodaira surface is not Kähler).

\subsection{Affine manifolds with singularities.}

Definition 1.15. An affine manifold with singularities is a topological manifold $B$ along with a closed set $\Delta \subseteq B$ which is locally a finite 
union of locally closed submanifolds of codimension at least 2 , and an affine structure on $B_{0}=B \backslash \Delta$. An affine manifold with singularities is integral if the affine structure on $B_{0}$ is integral. We always denote by $i: B_{0} \rightarrow B$ the inclusion map. A continuous map $f: B \rightarrow B^{\prime}$ of (integral) affine manifolds with singularities is (integral) affine if $f^{-1}\left(B_{0}^{\prime}\right) \cap B_{0}$ is dense in $B$ and

$$
\left.f\right|_{f^{-1}\left(B_{0}^{\prime}\right) \cap B_{0}}: f^{-1}\left(B_{0}^{\prime}\right) \cap B_{0} \rightarrow B_{0}^{\prime}
$$

is (integral) affine.

Example 1.16. We give a variety of two-dimensional examples.

1) Let $B=\{z \in \mathbb{C}|| z \mid<1\}, B_{0}=B \backslash\{0\}$. Let $\mathcal{H} \rightarrow B_{0}$ be the universal cover, where $\mathcal{H}$ is the upper half-plane with coordinate $w$ and covering map given by $w \mapsto e^{2 \pi i w}$. Then

$$
\delta(w)=\left(\operatorname{Re}\left(e^{2 \pi i w}\right), \operatorname{Re}\left(n e^{2 \pi i w}\left(w-\frac{1}{2 \pi i}\right)\right)\right)
$$

defines an affine structure on $B_{0}$ (this is the developing map of $\mathcal{H}$ into $\left.\mathbb{R}^{2}\right)$. If $\gamma$ is a counterclockwise simple loop around the origin, then $\rho(\gamma)$ is linear, given by the matrix $\left(\begin{array}{cc}1 & 0 \\ -n & 1\end{array}\right)$.

To see how this affine structure arises, consider the family $f_{0}: B_{0} \times$ $\mathbb{C} /\langle 1, \tau(z)\rangle \rightarrow B_{0}$ of elliptic curves over $B_{0}$ with period $\tau(z)=\frac{n}{2 \pi i} \log z$. This elliptic curve can be compactified over $B$ by adding a Kodairatype $I_{n}$ fibre, i.e., a cycle of $n$ projective lines. Let $y$ be the fibre coordinate in this family: then $d z \wedge d y$ is a holomorphic 2-form, and $\operatorname{Re}(d z \wedge d y)$ is a symplectic form, making the elliptic fibration into a Lagrangian fibration. The Arnold-Liouville Theorem yields an affine structure on $B_{0}$. A choice of affine coordinates $x_{1}, x_{2}$ in a neighbourhood $U \subseteq B_{0}$ is given by a choice of continuously varying local basis $\gamma_{1}, \gamma_{2}$ for $H_{1}\left(f^{-1}(b), \mathbb{Z}\right)$ for $b \in U$. Then at $b \in U$,

$$
\begin{aligned}
d x_{j}=d z_{1}\left(\left.\int_{\gamma_{j}}\left(\iota\left(\partial / \partial z_{1}\right) \operatorname{Re}(d z \wedge d y)\right)\right|_{f_{0}^{-1}(b)}\right) & \\
& +d z_{2}\left(\left.\int_{\gamma_{j}}\left(\iota\left(\partial / \partial z_{2}\right) \operatorname{Re}(d z \wedge d y)\right)\right|_{f_{0}^{-1}(b)}\right),
\end{aligned}
$$

for $z=z_{1}+i z_{2}$. (See [16], $\S 2$ for more details concerning the ArnoldLiouville theorem in the special Lagrangian situation). Taking $\gamma_{1}$ to be given by the period 1 and $\gamma_{2}$ to be given by the period $\tau(z)$, then $d x_{1}=\operatorname{Re}(d z)$ and $d x_{2}=\operatorname{Re}(\tau(z) d z)$, so we can take $x_{1}=\operatorname{Re} z$ and $x_{2}=\operatorname{Re}\left(\frac{n}{2 \pi i}(z \log z-z)\right)$ as given.

We note that $\operatorname{Re} z=0$ gives a well-defined line through the origin. This allows one to identify this affine structure in a neighbourhood of zero for $n=1$ with a neighbourhood of the singularity defined in (2). 
2) Next we will give an affine manifold (with boundary) with singularities by gluing together polyhedra. The affine manifold will be a union of two triangles, as depicted in either the left or right hand pictures below, but we define the affine structure by drawing the affine embedding of two open sets covering $B$, obtained by making cuts as shown. Here the solid lines denote cuts, the " $\times$ " being the singular point, and affine coordinates are given at the vertices of the two triangles. Thus the two pictures give two systems of affine coordinates, linear on each triangle. The intersection of the two coordinate charts is just $B$ minus the common edge of the two triangles. On the left-hand component the change of coordinates is the identity, but on the right-hand component the change of coordinates is given by $\left(x_{1}, x_{2}\right) \mapsto\left(x_{1}, x_{1}+x_{2}\right)$. Alternatively, the right-hand triangles in the left and right-hand pictures are the same, identified by the linear transformation $\left(\begin{array}{ll}1 & 0 \\ 1 & 1\end{array}\right)$.

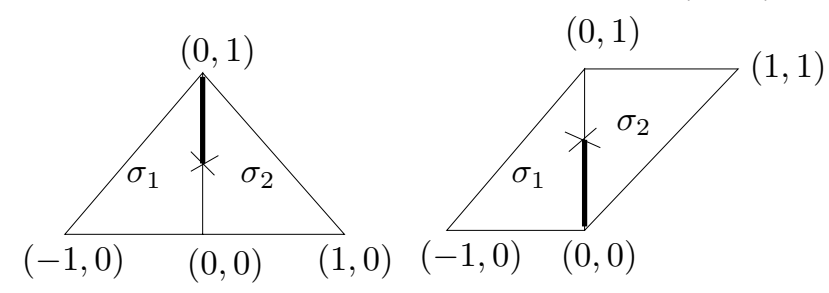

3) The dual intersection complex of the quartic degeneration given in the introduction is an integral affine manifold with singularities.

4) A cone with a cone angle $0<\alpha \leq 2 \pi$ is an example of an affine manifold with singularities: take the wedge $\{z \in \mathbb{C} \mid 0 \leq \arg z \leq \alpha\}$ in $\mathbb{C}$, and identify the two edges via rotation. The holonomy is given by rotation by the angle $-\alpha$, i.e.,

$$
\left(\begin{array}{cc}
\cos \alpha & \sin \alpha \\
-\sin \alpha & \cos \alpha
\end{array}\right)
$$

Such a rotation is integral with respect to any lattice in $\mathbb{C}$ if $\alpha=\pi$, is integral with respect to the lattice $\mathbb{Z}[i] \subseteq \mathbb{C}$ if $\alpha=n \pi / 2,1 \leq n<4$, and is integral with respect to the lattice $\mathbb{Z}[(1+i \sqrt{3}) / 2]$ if $\alpha=n \pi / 3$, $1 \leq n<6$. In no other cases is the cone integral.

In the case these affine structures are integral, these are all interesting singularities. However, in this paper, the only two-dimensional singularities we will deal with in our general construction will be those occuring in (1)-(3). In particular, the examples of (4) do not have polyhedral decompositions in the sense defined in the next section. A more general approach to building degenerations from affine manifolds with singularities should also deal with singularities of the second type. This seems possible in two dimensions, and there is some hope of generalising this to higher dimensions. 
Example 1.17. Let $f: X \rightarrow \mathbb{P}^{1}$ be an elliptically fibred K3 surface with holomorphic 2-form $\Omega=\Omega_{1}+i \Omega_{2}$. The fibres of $f$ are Lagrangian with respect to both $\Omega_{1}$ and $\Omega_{2}$. As in Example 1.16, (1), by the ArnoldLiouville theorem, one then obtains from each of these symplectic forms an affine structure on $B_{0}=\mathbb{P}^{1} \backslash \Delta$, where $\Delta$ is the discriminant locus of $f$. These affine structures will in fact be related by a Legendre transform. Furthermore, if $\left[\Omega_{i}\right] \in H^{2}(X, \mathbb{Z})$, then one can show the affine structure induced by $\Omega_{i}$ will be integral.

Example 1.18. Let $\Xi \subseteq M_{\mathbb{R}} \cong \mathbb{R}^{n}$ be a polytope containing 0 in its interior, and let $B=\partial \Xi$. Denote by $\operatorname{Bar}(B)$ the first barycentric subdivision of the boundary of $\Xi$ (see Definition 1.25). Let $\Delta$ be the union of the simplices in $\operatorname{Bar}(B)$ not containing any vertex of $\Xi$ nor containing the barycenter of any $n$-1-dimensional face of $\Xi$. Set $B_{0}=$ $B \backslash \Delta$.

We can define an affine structure on $B_{0}$ as follows. For every $n-1$ dimensional face $\sigma$ of $\Xi$, let $W_{\sigma}=\operatorname{Int}(\sigma)$. For each vertex $v$ of $\Xi$, let $\check{v} \subseteq B$ be the union of all simplices of $\operatorname{Bar}(B)$ containing $v$, and set $W_{v}=\operatorname{Int}(\check{v})$. Then $\left\{W_{\sigma}\right\} \cup\left\{W_{v}\right\}$ form an open cover for $B_{0}$. We can then define affine charts for each $n$-1-dimensional face $\sigma$,

$$
\psi_{\sigma}: W_{\sigma} \longrightarrow \mathbb{A}_{\sigma} \subseteq M_{\mathbb{R}}
$$

to be the inclusion of $\sigma$ inside the affine hyperplane $\mathbb{A}_{\sigma}$ spanned by $\sigma$. For a vertex $v$, define a chart

$$
\varphi_{v}: W_{v} \longrightarrow M_{\mathbb{R}} / \mathbb{R} v
$$

by projection. It is a simple exercise to show this defines an affine structure on $B_{0}$, which is integral when $\Xi$ is a reflexive lattice polytope [6]. See [23] for a generalisation of this construction.

Example 1.19. Let $M=\mathbb{Z}^{4}+\frac{1}{5}(1,2,3,4)$, and let $\Xi \subseteq M_{\mathbb{R}}$ be the polytope with vertices

$$
(-1,-1,-1,-1),(1,0,0,0),(0,1,0,0),(0,0,1,0),(0,0,0,1) .
$$

(Adding the fractional lattice point is not necessary for this example, but will be used in Example 4.29.) By Example 1.18, we obtain an integral affine manifold with singularities structure on $\partial \Xi$. Furthermore, the group $\mathbb{Z} / 5 \mathbb{Z}$ acts on $M$ with generator

$$
\left(\begin{array}{llll}
0 & 0 & 0 & -1 \\
1 & 0 & 0 & -1 \\
0 & 1 & 0 & -1 \\
0 & 0 & 1 & -1
\end{array}\right) .
$$

This cyclically permutes the vertices of $\Xi$, and one can check that it induces integral affine automorphisms of the boundary. Thus if $B=$ $\partial \Xi /(\mathbb{Z} / 5 \mathbb{Z}), B$ is also an integral affine manifold with singularities. In this case $B$ is in fact a lens space. 
Example 1.20. Let $\Xi \subseteq M_{\mathbb{R}}=\mathbb{R}^{3}$ be the octahedron with vertices

$$
( \pm 1,0,0),(0, \pm 1,0),(0,0, \pm 1) \text {. }
$$

Then $\mathbb{Z} / 2 \mathbb{Z}$ acts on $\Xi$ by negation, and thus acts on $\partial \Xi$ by affine transformations. Take $B=\partial \Xi /(\mathbb{Z} / 2 \mathbb{Z})$. This is a real projective plane, with six singular points.

1.3. Polyhedral decompositions. We would like to define a polyhedral decomposition of an affine manifold $B$ with singularities. Intuitively, we want this to be a cell decomposition of $B$ into polyhedra in affine space. There are two subtleties which make the definition slightly complicated. The first is that we would like our cells to be able to be self-intersecting: for example, if $B=\mathbb{R} / \mathbb{Z}$, we would like to take $B$ to be a maximal cell (viewed as the interval $[0,1]$ with endpoints identified) and $0 \bmod \mathbb{Z}$ a zero-dimensional cell. This is the dual intersection complex of a nodal elliptic curve, and we do not wish to rule out such a basic example. As a result, we first define a polyhedral decomposition of a region in $M_{\mathbb{R}}$, and then use this definition locally on $B$. Secondly, there is some subtlety in how cells are allowed to interact with the discriminant locus, and this will show up in the additional restriction given below for toric polyhedral decompositions.

Definition 1.21. A polyhedral decomposition of a closed set $R \subseteq M_{\mathbb{R}}$ is a locally finite covering $\mathscr{P}$ of $R$ by closed convex polytopes (called cells) with the property that

1) if $\sigma \in \mathscr{P}$ and $\tau \subseteq \sigma$ is a face then $\tau \in \mathscr{P}$;

2) if $\sigma, \sigma^{\prime} \in \mathscr{P}$, then $\sigma \cap \sigma^{\prime}$ is a common face of $\sigma$ and $\sigma^{\prime}$.

We say the decomposition is integral if all vertices (0-dimensional elements of $\mathscr{P}$ ) are contained in $M$.

For a polyhedral decomposition $\mathscr{P}$ and $\sigma \in \mathscr{P}$ we define the (relative) interior of $\sigma$ as

$$
\operatorname{Int}(\sigma)=\sigma \backslash \bigcup_{\tau \in \mathscr{P}, \tau \subsetneq \sigma} \tau .
$$

Definition 1.22. Let $B$ be an integral affine manifold with singularities. A polyhedral decomposition of $B$ is a collection $\mathscr{P}$ of closed subsets of $B$ (called cells) covering $B$ which satisfies the following properties. If $\{v\} \in \mathscr{P}$ for some point $v \in B$, then $v \notin \Delta$ and there exists an integral polyhedral decomposition $\mathscr{P}_{v}$ of a closed neighbourhood of the origin $R_{v} \subseteq \Lambda_{\mathbb{R}, v} \cong \mathcal{T}_{B, v}$ (the stalk of the local system $\Lambda_{\mathbb{R}}$ at $v$ or equivalently the tangent space of $B$ at $v$ ) which is the closure of an open neighbourhood of the origin, and a continuous map $\exp _{v}: R_{v} \rightarrow B, \exp _{v}(0)=v$, satisfying 
1) $\exp _{v}$ is locally a homeomorphism onto its image, is injective on $\operatorname{Int}(\tau)$ for all $\tau \in \mathscr{P}_{v}$, and is an integral affine map in some neighbourhood of the origin.

2) For every top-dimensional $\tilde{\sigma} \in \mathscr{P}_{v}, \exp _{v}(\operatorname{Int}(\tilde{\sigma})) \cap \Delta=\emptyset$ and the restriction of $\exp _{v}$ to $\operatorname{Int}(\tilde{\sigma})$ is integral affine. Furthermore, $\exp _{v}(\tilde{\tau}) \in \mathscr{P}$ for all $\tilde{\tau} \in \mathscr{P}_{v}$.

3) $\sigma \in \mathscr{P}$ and $v \in \sigma \Leftrightarrow \sigma=\exp _{\mathrm{v}}(\tilde{\sigma})$ for some $\tilde{\sigma} \in \mathscr{P}_{v}$ with $0 \in \tilde{\sigma}$.

4) Every $\sigma \in \mathscr{P}$ contains a point $v \in \sigma$ with $\{v\} \in \mathscr{P}$.

In addition we say the polyhedral decomposition is toric if it satisfies the additional condition

5) For each $\sigma \in \mathscr{P}$, there is a neighbourhood $U_{\sigma} \subseteq B$ of $\operatorname{Int}(\sigma)$ and an integral affine submersion $S_{\sigma}: U_{\sigma} \rightarrow M_{\mathbb{R}}^{\prime}$ where $M^{\prime}$ is a lattice of rank equal to $\operatorname{dim} B-\operatorname{dim} \sigma$ and $S_{\sigma}\left(\sigma \cap U_{\sigma}\right)=\{0\}$.

We will write $\mathscr{P}_{\max }=\{\sigma \in \mathscr{P} \mid \sigma$ is maximal, i.e., $\operatorname{dim} \sigma=\operatorname{dim} B\}$.

\section{Example 1.23.}

1) If $B=M_{\mathbb{R}}, \Delta=\emptyset$, a polyhedral decomposition of $B$ is just an integral polyhedral decomposition of $M_{\mathbb{R}}$ in the sense of Definition 1.21. In this case, when the discriminant locus $\Delta$ is empty, the toric condition is vacuous. If $B=M_{\mathbb{R}} / \Gamma$ for some lattice $\Gamma$, then a polyhedral decomposition of $B$ is induced by a polyhedral decomposition of $M_{\mathbb{R}}$ invariant under $\Gamma$. Similarly, in Example 1.5 (3), the same holds. For example, $M_{\mathbb{R}} / \Gamma$ has a polyhedral decomposition containing only one maximal cell, coming from a fundamental domain for the action of $\Gamma$ on $M_{\mathbb{R}}$.

2) When $B$ has singularities, the definition of toric polyhedral decomposition imposes some slightly subtle restrictions on how the cells of $\mathscr{P}$ interact with $\Delta$. In particular, it places strong conditions on the holonomy of $B$ locally near $\Delta$ : see Proposition 1.32. However, it is a useful exercise to verify in Example 1.18 that if one takes $\mathscr{P}$ to be the collection of all proper subfaces of $\Xi$, then $\mathscr{P}$ is a toric polyhedral decomposition of $B$. Furthermore, in Examples 1.19 and 1.20, this polyhedral decomposition on $\partial \Xi$ descends to a polyhedral decomposition of $B$. In particular, in the case of Example 1.19, there is only one cell of dimension three in the decomposition, and one vertex. One reason for the complexity of the definition of polyhedral decomposition is that we wish to allow cells to be self-intersecting, i.e., be polytopes with some sides identified. These examples show the necessity of this.

Remark 1.24. Given a polyhedral decomposition $\mathscr{P}$ on $B$, if $v$ is a vertex of $\mathscr{P}$, we can look at the polyhedral decomposition of $R_{v}$ in a small neighbourhood of the origin in $\Lambda_{\mathbb{R}, v}$. This clearly coincides with a small neighbourhood of the origin of a complete rational polyhedral fan $\Sigma_{v}$ in $\Lambda_{\mathbb{R}, v}$ : 


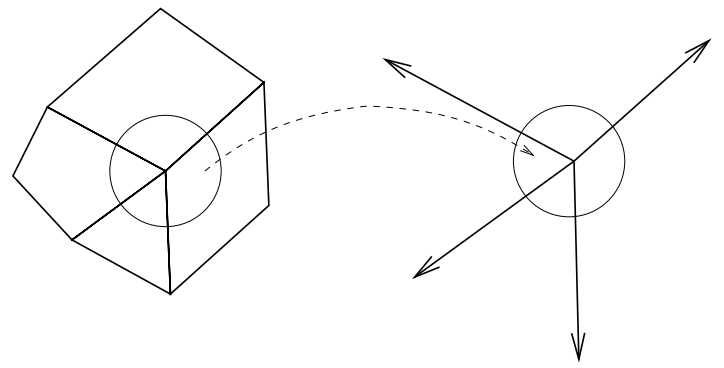

In fact, we shall shortly see that the data of the affine structures on maximal cells of $\mathscr{P}$ and a fan structure at each vertex $v$ essentially determine the affine structure on $B$.

Definition 1.25. Recall that if $\sigma \subset M_{\mathbb{R}}$ is a polytope, then the barycenter $\operatorname{Bar}(\sigma)$ of $\sigma$ is the average of the vertices of $\sigma$, and thus is invariant under affine transformations. The first barycentric subdivision of $\sigma$ is then the triangulation of $\sigma$ consisting of all simplices spanned by barycenters of ascending chains of faces of $\sigma$. Thus given a polyhedral decomposition $\mathscr{P}$ of an affine manifold with singularities $B$, we can define the first barycentric subdivision $\operatorname{Bar}(\mathscr{P})$ of $\mathscr{P}$ to be the triangulation consisting of all images of simplices in the first barycentric subdivisions of all $\tilde{\sigma} \in \mathscr{P}_{v}$ for all vertices $v$. Because barycentric subdivisions are affine invariants, this gives a well-defined triangulation of $B$. Note that $\operatorname{Bar}(\mathscr{P})$ need not be a polyhedral decomposition of $B$ if $\Delta \neq \emptyset$. For example, a vertex of $\operatorname{Bar}(\mathscr{P})$ might be contained in $\Delta$.

For any $\tau \in \mathscr{P}$, let $W_{\tau}$ be the union of the interiors of all simplices of $\operatorname{Bar}(\mathscr{P})$ intersecting $\operatorname{Int}(\tau)$ (i.e., having the barycenter of $\tau$ as a vertex). This can be thought of as the (open) star of the barycenter of $\tau$ in the triangulation $\operatorname{Bar}(\mathscr{P})$ of $B$. Set $\mathscr{W}=\left\{W_{\tau} \mid \tau \in \mathscr{P}\right\}$; this is an open covering of $B$.

Construction 1.26. We will now describe a standard procedure for constructing affine manifolds with singularities along with polyhedral decompositions. Let $\mathscr{P}^{\prime}$ be a collection of $n$-dimensional integral polytopes in $M_{\mathbb{R}}$. Suppose we are given integral affine identifications of various proper faces of the polytopes in $\mathscr{P}^{\prime}$ in such a way that once we glue the polytopes using these identifications, we obtain a manifold $B$, along with a decomposition $\mathscr{P}$ consisting of images of faces of polytopes in $\mathscr{P}^{\prime}$. In particular, we have the identification map

$$
\pi: \coprod_{\sigma^{\prime} \in \mathscr{P}^{\prime}} \sigma^{\prime} \rightarrow B
$$

Now $B$ is not yet an affine manifold with singularities. It only has an affine structure defined in the interiors of maximal cells. When two polytopes of $\mathscr{P}^{\prime}$ are identified along faces, we have an affine structure on that face, but no affine structure in the directions "transversal" to that 
face. We cannot, however, expect an affine structure on all of $B$, and we need to choose a discriminant locus. This still does not give enough data to specify an affine structure on $B$ away from the discriminant locus. We will also need to choose a fan structure at each vertex of $\mathscr{P}$. We define these data below, but first define the discriminant locus.

Let $\operatorname{Bar}(\mathscr{P})$ be the first barycentric subdivision of $\mathscr{P}$. Then we define $\Delta^{\prime} \subseteq B$ to be the union of all simplices in $\operatorname{Bar}(\mathscr{P})$ not containing a vertex of $\mathscr{P}$ or the barycenter of a maximal cell of $\mathscr{P}$.

For a vertex $v$ of $\mathscr{P}$, let $W_{v}$ be the union of the interiors of all simplices in $\operatorname{Bar}(\mathscr{P})$ containing $v$. Then $W_{v}$ is an open neighbourhood of $v$, and

$$
\left\{W_{v} \mid v \text { a vertex of } \mathscr{P}\right\} \cup\left\{\operatorname{Int}(\sigma) \mid \sigma \in \mathscr{P}_{\max }\right\}
$$

forms an open covering of $B \backslash \Delta^{\prime}$. To define an affine structure on $B \backslash \Delta^{\prime}$, we need to choose affine charts on $W_{v}$.

For a vertex $v$ of $\mathscr{P}$, let

$$
\mathscr{P}_{v}^{\prime}=\left\{\left(v^{\prime}, \sigma^{\prime}\right) \mid v^{\prime} \in \sigma^{\prime} \in \mathscr{P}^{\prime} \text { a vertex, } \pi\left(v^{\prime}\right)=v\right\} .
$$

Let $R_{v}$ be the quotient of $\coprod_{\left(v^{\prime}, \sigma^{\prime}\right) \in \mathscr{P}_{v}^{\prime}} \sigma^{\prime}$ by the following equivalence relation. Let $\left(v_{i}^{\prime}, \sigma_{i}^{\prime}\right) \in \mathscr{P}_{v}^{\prime}$ and $y_{i} \in \sigma_{i}^{\prime}$ for $i=1,2$. Let $\omega_{i}^{\prime} \subseteq \sigma_{i}^{\prime}$ be the smallest subface of $\sigma_{i}^{\prime}$ containing $y_{i}$. Then $y_{1} \sim y_{2}$ if

1) $\pi\left(y_{1}\right)=\pi\left(y_{2}\right)$,

2) $v_{i}^{\prime} \in \omega_{i}^{\prime}$

3) $\pi$ identifies germs of $\omega_{1}^{\prime}$ at $v_{1}^{\prime}$ and $\omega_{2}^{\prime}$ at $v_{2}^{\prime}$. By this we mean there exist arbitrarily small open neighbourhoods $U_{i}$ of $v_{i}^{\prime}$ in $\omega_{i}^{\prime}$ such that $\pi\left(U_{1}\right)=\pi\left(U_{2}\right)$.

(This equivalence relation was not quite correct in [19].) For example, if $\mathscr{P}^{\prime}$ consists of the unit square in $\mathbb{R}^{2}$, and $B$ is obtained by identifying opposite sides, we have a unique vertex $v$ in $\mathscr{P}$ and the picture

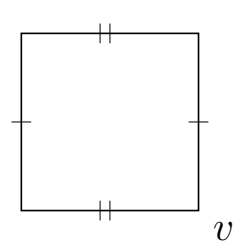

$B$

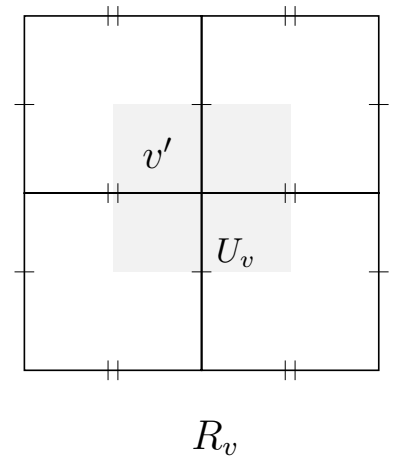

Condition (3) is necessary in this example, as otherwise there would be further identifications of the interior one-dimensional faces of $R_{v}$.

There is a continuous map

$$
\pi_{v}: R_{v} \longrightarrow B
$$


defined by taking $b \in \sigma^{\prime} \subseteq R_{v}$ to $\pi(b)$, and it is easy to see that if $U_{v}$ is the connected component of $\pi_{v}^{-1}\left(W_{v}\right)$ containing the equivalence class of points $\left\{v^{\prime} \in \sigma^{\prime} \mid\left(v^{\prime}, \sigma^{\prime}\right) \in \mathscr{P}_{v}^{\prime}\right\}$ in $R_{v}$, then $U_{v} \rightarrow W_{v}$ is a homeomorphism. We write this equivalence class of points as $v^{\prime}$.

$R_{v}$ has an abstract polyhedral decomposition $\mathscr{P}_{v}$, with $v^{\prime}$ the unique vertex in $U_{v}$ mapping to $v$. We will need to find an embedding $i_{v}: R_{v} \rightarrow$ $M_{\mathbb{R}}$. If this is done appropriately, then a coordinate chart $\psi_{v}: W_{v} \rightarrow$ $M_{\mathbb{R}}$ can be defined as $\left.i_{v} \circ \pi_{v}^{-1}\right|_{W_{v}}$, and $\exp _{v}: i_{v}\left(R_{v}\right) \rightarrow B$ can be defined as $\pi_{v} \circ i_{v}^{-1}$, giving both an affine structure on $B \backslash \Delta^{\prime}$ and a proof that $\mathscr{P}$ is a polyhedral decomposition of $B$.

To do this, we need to choose a fan structure at each vertex $v$ of $\mathscr{P}$. This means for each $v$ we choose a complete rational polyhedral fan $\Sigma_{v}$ in $M_{\mathbb{R}}$ (see [38] for a definition) and a one-to-one inclusion preserving correspondence between elements of $\mathscr{P}_{v}$ containing $v^{\prime}$ and elements of $\Sigma_{v}$ which we write as $\sigma \mapsto \sigma_{v^{\prime}}$. Furthermore, this correspondence should have the property that there exists an integral affine isomorphism $i_{\sigma}$ between the tangent wedge of $\sigma$ at $v^{\prime}$ and $\sigma_{v^{\prime}}$ which preserves the correspondence. Such an isomorphism, if it exists, is unique (it is determined by specifying what it does to primitive integral generators of each ray, and by integrality, these must be sent to primitive integral generators). By this uniqueness, the maps $i_{\sigma}$ glue together to give a map

$$
i_{v}: R_{v} \longrightarrow M_{\mathbb{R}}
$$

which is a homeomorphism onto its image. Then it is easy to see that using $\psi_{v}$ and $\exp _{v}$ as defined above one obtains an integral affine structure on $B_{0}:=B \backslash \Delta^{\prime}$ and one sees that $\mathscr{P}$ is a polyhedral decomposition.

Suppose we have carried out Construction 1.26, and so obtained an affine structure on $B \backslash \Delta^{\prime}$ and a polyhedral decomposition $\mathscr{P}$. It often happens that our choice of $\Delta^{\prime}$ is too crude, and we can still extend the affine structure to a larger open set of $B$. Our original discriminant locus $\Delta^{\prime}$ is a union of all codimension two simplices of $\operatorname{Bar}(\mathscr{P})$ not containing a vertex of $\mathscr{P}$ or the barycenter of a maximal cell of $\mathscr{P}$. Let $\tau$ be such a simplex. Locally at a point $b \in \operatorname{Int}(\tau), B \backslash \Delta^{\prime}$ takes the form $\mathbb{R}^{n-2} \times\left(\mathbb{R}^{2} \backslash\{(0,0)\}\right)$ topologically. We say the holonomy around the simplex $\tau$ is trivial if the holonomy of the affine structure on $B \backslash \Delta^{\prime}$ about a simple loop around the origin in $\mathbb{R}^{2} \backslash\{(0,0)\} \subset \mathbb{R}^{n-2} \times\left(\mathbb{R}^{2} \backslash\{(0,0)\}\right)$ is trivial.

Let $\Delta$ be the union of all codimension two simplices in $\Delta^{\prime}$ about which the holonomy is non-trivial.

Proposition 1.27. The affine structure on $B \backslash \Delta^{\prime}$ extends uniquely to an affine structure on $B \backslash \Delta$.

Proof. Let $\omega$ be a simplex of $\operatorname{Bar}(\mathscr{P})$ contained in $\Delta^{\prime}$ but not $\Delta$. Let $\tau$ be the minimal cell of $\mathscr{P}$ containing $\omega$. Then $0<\operatorname{dim} \tau<\operatorname{dim} B$. 
Choose a vertex $v$ of $\tau$ such that $\omega \subseteq \overline{W_{v}}$, and we obtain $R_{v} \subseteq \Lambda_{\mathbb{R}, v}$, $\mathscr{P}_{v}$ and $\exp _{v}: R_{v} \rightarrow B$. Let $\tilde{\tau} \in \mathscr{P}_{v}$ with $0 \in \tilde{\tau}$ and $\exp _{v}(\tilde{\tau})=\tau$. Let $U_{v}$ be the connected component of $\exp _{v}^{-1}\left(W_{v}\right)$ containing 0 , and let $\tilde{\omega} \subseteq \tilde{\tau} \cap \overline{U_{v}}$ be such that $\exp _{v}(\tilde{\omega})=\omega$. Then $\tilde{\tau}$ is contained in maximal cells $\tilde{\sigma}_{1}, \ldots, \tilde{\sigma}_{m} \in \mathscr{P}_{v}$. Let $U$ be a small open neighbourhood of $\operatorname{Int}(\tilde{\omega})$ which is contained in $\bigcup \tilde{\sigma}_{i}$ and such that $\exp _{v}(U)$ is disjoint from any simplex in $\Delta^{\prime}$ not containing $\omega$. Then $U \subseteq \Lambda_{\mathbb{R}, v}$ inherits an integral affine structure.

Let $U^{\prime}=U \backslash \exp _{v}^{-1}\left(\Delta^{\prime}\right)$. If we can show $\left.\exp _{v}\right|_{U^{\prime}}$ is an integral affine isomorphism onto its image in $B \backslash \Delta^{\prime}$, then we can patch $U$ and $B \backslash \Delta^{\prime}$ and so extend the integral affine structure across $\operatorname{Int}(\omega)$.

It is clear from our choice of $U$ that $\left.\exp _{v}\right|_{U^{\prime}}$ is a homeomorphism onto its image, and by construction it is integral affine on

$$
U^{\prime} \cap\left(U_{v} \cup \operatorname{Int}\left(\tilde{\sigma}_{1}\right) \cup \cdots \cup \operatorname{Int}\left(\tilde{\sigma}_{m}\right)\right) .
$$

If $y \in U^{\prime} \backslash\left(U_{v} \cup \operatorname{Int}\left(\tilde{\sigma}_{1}\right) \cup \cdots \cup \operatorname{Int}\left(\tilde{\sigma}_{m}\right)\right)$, we need to show $\exp _{v}$ is integral affine at $y$. Let $V$ be a small open neighbourhood of $y$ in $U^{\prime}$, mapping $V$ onto an open set $\exp _{v}(V)$ which we can identify via its affine structure

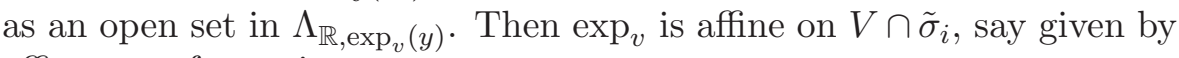
affine transformations

$$
\psi_{i}: \Lambda_{\mathbb{R}, v} \longrightarrow \Lambda_{\mathbb{R}, \exp _{v}(y)} .
$$

Let $\gamma$ be the image of a loop based at $y$ which passes from $y$ into $\tilde{\sigma}_{i}$ to 0 , then into $\tilde{\sigma}_{j}$ and back to $y$. Then up to conjugation by a translation, $\rho(\gamma)=\psi_{i} \circ \psi_{j}^{-1}$. Now $\gamma$ can be viewed as a loop around a codimension 2 simplex of $\Delta^{\prime}$ containing $\omega$, so $\rho(\gamma)$ is assumed to be trivial. This says $\psi_{i}=\psi_{j}$. This holds for all $1 \leq i, j \leq m$ and so $\exp _{v}$ is affine at $y$ coinciding with $\psi_{i}$ for any $i$.

Uniqueness follows since a map between connected open subsets of $\mathbb{R}^{n}$ can be checked to be affine on any connected, dense open subset. q.e.d.

Definition 1.28. Let $B$ be an integral affine manifold with singularities and a polyhedral decomposition $\mathscr{P}$. We define a sheaf $\Lambda_{\mathscr{P}, \mathbb{R}}$ on $B_{0}$ as a subsheaf of $\Lambda_{\mathbb{R}}$ by setting

$$
\Gamma\left(U, \Lambda_{\mathscr{P}, \mathbb{R}}\right)=\left\{v \in \Gamma\left(U, \Lambda_{\mathbb{R}}\right) \mid \begin{array}{c}
\forall y \in U, \sigma \in \mathscr{P} \text { with } y \in \sigma, \\
v \text { is tangent to } \sigma \text { at } y
\end{array}\right\} .
$$

Similarly, we can define $\Lambda_{\mathscr{P}}$ as above as a subsheaf of $\Lambda$. Note that if $y \in \operatorname{Int}(\sigma)$, the stalk $\left(\Lambda_{\mathscr{P}, \mathbb{R}}\right)_{y}$ is the tangent space to $\sigma$ at $y$, and $\Lambda_{\mathscr{P}, y}$ is a lattice in $\left(\Lambda_{\mathscr{P}, \mathbb{R}}\right)_{y}$.

The existence of a polyhedral decomposition of an affine manifold with singularities places restrictions on the nature of its singularities, and whether the polyhedral decomposition is toric can be detected via its holonomy. 
Proposition 1.29. Let $\mathscr{P}$ be a polyhedral decomposition of $B$, an integral affine manifold with singularities. For any $\tau \in \mathscr{P}$, there exists an open neighbourhood $U_{\tau}$ of $\operatorname{Int}(\tau)$ such that if $y \in \operatorname{Int}(\tau) \backslash \Delta$ and

$$
\rho: \pi_{1}\left(U_{\tau} \backslash \Delta, y\right) \longrightarrow \operatorname{Aff}\left(\Lambda_{\mathbb{R}, y}\right)
$$

is the local holonomy representation,

$$
\tilde{\rho}: \pi_{1}\left(U_{\tau} \backslash \Delta, y\right) \longrightarrow \operatorname{GL}\left(\Lambda_{\mathbb{R}, y}\right)
$$

the linear part of the holonomy representation (hence the monodromy representation for the local system $\Lambda$ ), then the radiance obstruction of $\rho$ is trivial and

$$
(\tilde{\rho}(\gamma)-I)\left(\left(\Lambda_{\mathscr{P}, \mathbb{R}}\right)_{y}\right)=0
$$

for all $\gamma \in \pi_{1}\left(U_{\tau} \backslash \Delta, y\right)$.

Proof. Let $v$ be a vertex of $\tau, R_{v}, \mathscr{P}_{v}$ and $\exp _{v}$ as usual, and let $\tilde{\tau} \in \mathscr{P}_{v}$ with $0 \in \tilde{\tau}$ and $\exp _{v}(\tilde{\tau})=\tau$. Let $\tilde{U}_{\tau}$ be a sufficiently small open neighbourhood of $\operatorname{Int}(\tilde{\tau})$ such that $\exp _{v}$ maps $\tilde{U}_{\tau}$ homeomorphically to an open neighbourhood $U_{\tau}$ of $\operatorname{Int}(\tau)$. Let $\tilde{y} \in R_{v}$ map to $y \in \operatorname{Int}(\tau) \backslash \Delta$. Consider a loop in $U_{\tau} \backslash \Delta$ based at $y$, which we can lift to a loop $\tilde{\gamma}$ in $\tilde{U}_{\tau}$ based at $\tilde{y}$. We can always assume, by deforming $\gamma$, that $\tilde{\gamma}$ intersects non-maximal cells of $\mathscr{P}_{v}$ only in a finite number of points. Indexing these points by $\mathbb{Z} / m \mathbb{Z}$, we write them as $\tilde{y}=\tilde{z}_{0}, \tilde{z}_{1}, \ldots, \tilde{z}_{m-1}$ in order through which $\tilde{\gamma}$ passes through them, so that we arrive at $\tilde{z}_{m}=\tilde{z}_{0}$ when we have finished traversing $\tilde{\gamma}$. Let $\tilde{\sigma}_{i} \in \mathscr{P}_{v}$ be the maximal cell that $\tilde{\gamma}$ passes through between $\tilde{z}_{i}$ and $\tilde{z}_{i+1}$. Finally, let $z_{i}=\exp _{v}\left(\tilde{z}_{i}\right)$. Identifying a small open neighbourhood $V_{i}$ of $z_{i}$ with an open neighbourhood of the origin in $\Lambda_{\mathbb{R}, z_{i}}$, the map $\exp _{v}$ is given by affine maps

$$
\begin{aligned}
& \psi_{i}^{i-1}: \Lambda_{\mathbb{R}, v} \longrightarrow \Lambda_{\mathbb{R}, z_{i}} \\
& \psi_{i}^{i}: \Lambda_{\mathbb{R}, v} \longrightarrow \Lambda_{\mathbb{R}, z_{i}}
\end{aligned}
$$

on $\exp _{v}^{-1}\left(V_{i}\right) \cap \tilde{\sigma}_{i-1}$ and $\exp _{v}^{-1}\left(V_{i}\right) \cap \tilde{\sigma}_{i}$ respectively. If $\tilde{z}_{i} \in \operatorname{Int}\left(\tilde{\omega}_{i}\right)$ for some cell $\tilde{\omega}_{i} \in \mathscr{P}_{v}$, then $\psi_{i}^{i-1}$ and $\psi_{i}^{i}$ must agree on the linear space $\mathbb{R} \tilde{\omega}_{i}$ spanned by $\tilde{\omega}_{i}$, and since we can assume $U_{\tau}$ is sufficiently small, we can assume $\mathbb{R} \tilde{\omega}_{i}$ contains $\mathbb{R} \tilde{\tau}$. Thus $\left(\psi_{i}^{i}\right)^{-1} \circ \psi_{i}^{i-1}$ is the identity on $\mathbb{R} \tilde{\tau}$, while $\psi_{0}^{m-1}$ and $\psi_{0}^{0}$ identify $\mathbb{R} \tilde{\tau}$ with $\left(\Lambda_{\mathscr{P}, \mathbb{R}}\right)_{y}$. Now we see that

$$
\rho(\gamma)^{-1}=\psi_{0}^{m-1} \circ\left(\psi_{m-1}^{m-1}\right)^{-1} \circ \psi_{m-1}^{m-2} \circ \cdots \circ\left(\psi_{1}^{1}\right)^{-1} \circ \psi_{1}^{0} \circ\left(\psi_{0}^{0}\right)^{-1},
$$

which is the identity on $\left(\Lambda_{\mathscr{P}, \mathbb{R}}\right)_{y}$. In particular, $0 \in\left(\Lambda_{\mathscr{P}, \mathbb{R}}\right)_{y}$ is fixed by $\rho(\gamma)$ so the translational part of $\rho(\gamma)$ is zero and the radiance obstruction vanishes.

q.e.d.

Remark 1.30. This local triviality of the radiance obstruction can be viewed as saying that the radiance obstruction $c_{B_{0}} \in H^{1}\left(B_{0}, \Lambda\right)$ is in the image of the inclusion $H^{1}\left(B, i_{*} \Lambda\right) \hookrightarrow H^{1}\left(B_{0}, \Lambda\right)$ induced by the 
Leray spectral sequence for $i: B_{0} \hookrightarrow B$. Indeed, local triviality says that the radiance obstruction is zero in $H^{0}\left(B, R^{1} i_{*} \Lambda\right)$.

Definition 1.31. Let $B$ be an integral affine manifold with singularities with a polyhedral decomposition $\mathscr{P}$. If $\sigma \in \mathscr{P}$, then the subspaces $\left(\Lambda_{\mathscr{P}, \mathbb{R}}\right)_{y}$ of $\Lambda_{\mathbb{R}, y}$ for all $y \in \operatorname{Int}(\sigma) \backslash \Delta$ are canonically identified via parallel transport in a neighbourhood of $\operatorname{Int}(\sigma)$ by Proposition 1.29. For any $y \in \operatorname{Int}(\sigma) \backslash \Delta$ we denote this subspace of $\Lambda_{\mathbb{R}, y}$ by $\Lambda_{\sigma, \mathbb{R}}$. Similarly, we denote by $\Lambda_{\sigma}$ the corresponding subspace of $\Lambda_{y}$ for $y \in \operatorname{Int}(\sigma) \backslash \Delta$.

Proposition 1.32. Let $\mathscr{P}$ be a polyhedral decomposition of $B$. Then $\mathscr{P}$ is toric if and only if for each $\tau \in \mathscr{P}$, there exists an open neighbourhood $U_{\tau}$ of $\operatorname{Int}(\tau)$ such that if $y \in \operatorname{Int}(\tau) \backslash \Delta$ with

$$
\rho: \pi_{1}\left(U_{\tau} \backslash \Delta, y\right) \longrightarrow \operatorname{Aff}\left(\Lambda_{\mathbb{R}, y}\right),
$$

the local holonomy representation, then $(\tilde{\rho}(\gamma)-I)\left(\Lambda_{\mathbb{R}, y}\right) \subseteq \Lambda_{\tau, \mathbb{R}}$.

Proof. First suppose $(\tilde{\rho}(\gamma)-I)\left(\Lambda_{\mathbb{R}, y}\right) \subseteq \Lambda_{\tau, \mathbb{R}}$ in $U_{\tau}$, and we will show, possibly after shrinking $U_{\tau}$, that there is an affine submersion $S_{\tau}: U_{\tau} \rightarrow \Lambda_{\mathbb{R}, y} / \Lambda_{\tau, \mathbb{R}}$ with $S_{\tau}(\operatorname{Int}(\tau))=0$, thus showing $\mathscr{P}$ is toric. Let $v \in \tau$ be a vertex, $R_{v}, \mathscr{P}_{v}$ as usual, with $\tilde{\tau} \in \mathscr{P}_{v}$ with $0 \in \tilde{\tau}$ and $\exp _{v}(\tilde{\tau})=\tau$. Possibly by shrinking $U_{\tau}$, we can assume there is an open neighbourhood of $\operatorname{Int}(\tilde{\tau}), \tilde{U}_{\tau} \subseteq R_{v}$, with $\exp _{v}$ a homeomorphism of $\tilde{U}_{\tau}$ onto $U_{\tau}$. If we choose $v$ to be in the same connected component of $\tau \backslash \Delta$ as $y$, we can identify $\Lambda_{\mathbb{R}, y}$ and $\Lambda_{\mathbb{R}, v}$ by parallel transport along a path in $\tau$, and under this identification, the linear space $\mathbb{R} \tilde{\tau}$ spanned by $\tilde{\tau}$ is identified with $\Lambda_{\tau, \mathbb{R}}$.

We can then define

$$
S_{\tau}=p_{\tau} \circ \exp _{v}^{-1}
$$

where $p_{\tau}: \Lambda_{\mathbb{R}, v} \rightarrow \Lambda_{\mathbb{R}, v} / \mathbb{R} \tilde{\tau}$ is the projection. This is a continuous map, and clearly $S_{\tau}(\operatorname{Int}(\tau))=0$, so we just need to show $S_{\tau}$ is an affine submersion.

To do so, it suffices to show it is an affine submersion at any point $y^{\prime} \in \operatorname{Int}(\tau) \backslash \Delta$. Identify a small neighbourhood $V$ of such a point with a neighbourhood in $\Lambda_{\mathbb{R}, y^{\prime}}$. If $\tilde{\sigma}_{1}, \ldots, \tilde{\sigma}_{r}$ are the maximal cells of $\mathscr{P}_{v}$ containing $\tilde{\tau}$, then $\exp _{v}$ is given by an affine map $\psi_{i}: \Lambda_{\mathbb{R}, v} \rightarrow \Lambda_{\mathbb{R}, y^{\prime}}$ on $\tilde{\sigma}_{i} \cap V$. On the other hand, holonomy around a simple loop based at $y$ into $\tilde{\sigma}_{i}$ through $y^{\prime}$ into $\tilde{\sigma}_{j}$ back to $y$ is given by $\psi_{i}^{-1} \circ \psi_{j}$, so by assumption on the local holonomy, it is clear that $p_{\tau} \circ \psi_{i}^{-1}=p_{\tau} \circ \psi_{j}^{-1}$. Thus $S_{\tau}$ is affine at $y^{\prime}$, as $S_{\tau}$ coincides with the affine submersion $p_{\tau} \circ \psi_{i}^{-1}$ for any $i$.

Conversely, suppose we have an affine submersion $S_{\tau}: U_{\tau} \rightarrow M_{\mathbb{R}}^{\prime}$ as in Definition 1.22, (5). Then for $y \in \operatorname{Int}(\tau) \backslash \Delta, S_{\tau *}: \Lambda_{\mathbb{R}, y} \rightarrow M_{\mathbb{R}}^{\prime}$ clearly yields a factorization of $S_{\tau *}$ as $\Lambda_{\mathbb{R}, y} \rightarrow \Lambda_{\mathbb{R}, y} / \Lambda_{\tau, \mathbb{R}} \stackrel{\cong}{\longrightarrow} M_{\mathbb{R}}^{\prime}$. Thus on $U_{\tau} \backslash \Delta$, the sheaf kernel of $S_{\tau *}: \Lambda_{\mathbb{R}} \rightarrow M_{\mathbb{R}}^{\prime}$ is just obtained from $\Lambda_{\tau, \mathbb{R}}$ by 
parallel transport. Thus for any $\gamma \in \pi_{1}\left(U_{\tau} \backslash \Delta, y\right)$, as $\tilde{\rho}(\gamma)\left(\Lambda_{\tau, \mathbb{R}}\right)=\Lambda_{\tau, \mathbb{R}}$, the transformation $\tilde{\rho}(\gamma)$ induces the identity on $\Lambda_{\mathbb{R}, y} / \Lambda_{\tau, \mathbb{R}} \cong M_{\mathbb{R}}^{\prime}$. Thus $(\tilde{\rho}(\gamma)-I)\left(\Lambda_{\mathbb{R}, y}\right) \subseteq \Lambda_{\tau, \mathbb{R}}$ as desired.

q.e.d.

There will also be information in the normal directions of a cell $\tau \in \mathscr{P}$, for example the fan $\Sigma_{\tau}$ introduced in Definition 1.35 below. The normal spaces of the cells also glue into a sheaf, but now special care has to be taken with the discriminant locus because of monodromy. The following definition turns out to work best.

Definition 1.33. Let $B$ be an integral affine manifold with singularities and a toric polyhedral decomposition $\mathscr{P}$. We define a subsheaf $\mathcal{Q}_{\mathscr{P}, \mathbb{R}}$ of $i_{*}\left(\Lambda_{\mathbb{R}} / \Lambda_{\mathscr{P}, \mathbb{R}}\right)$ as follows. A section $s \in \Gamma\left(U, i_{*}\left(\Lambda_{\mathbb{R}} / \Lambda_{\mathscr{P}, \mathbb{R}}\right)\right)$ is a section of $\mathcal{Q}_{\mathscr{P}, \mathbb{R}}$ if there is an open cover $\left\{U_{i}\right\}$ of $U$ such that for any $i$ and for any path $\gamma:[0,1] \rightarrow U_{i} \backslash \Delta, \gamma^{*} s \in \Gamma\left([0,1], \gamma^{*}\left(\Lambda_{\mathbb{R}} / \Lambda_{\mathscr{P}, \mathbb{R}}\right)\right)$ is induced by a section $t \in \Gamma\left([0,1], \gamma^{*} \Lambda_{\mathbb{R}}\right)$ (so local sections are constant under parallel transport). We similarly define a subsheaf $\mathcal{Q}_{\mathscr{P}}$ of $i_{*}\left(\Lambda / \Lambda_{\mathscr{P}}\right)$.

We note that if $y \in \operatorname{Int}(\sigma)$ for some $\sigma \in \mathscr{P}$, and $U$ is a connected open neighbourhood of $y$ disjoint from any $\tau \in \mathscr{P}$ with $\sigma \nsubseteq \tau$, then

$$
\Gamma\left(U, \mathcal{Q}_{\mathscr{P}, \mathbb{R}}\right)=\Lambda_{\mathbb{R}, y^{\prime}} / \Lambda_{\sigma, \mathbb{R}}
$$

and

$$
\Gamma\left(U, \mathcal{Q}_{\mathscr{P}}\right)=\Lambda_{y^{\prime}} / \Lambda_{\sigma}
$$

where $y^{\prime} \in \sigma \cap(U \backslash \Delta)$. By Proposition 1.32, this is independent of the choice of $y^{\prime}$. We will write

$$
\begin{aligned}
\mathcal{Q}_{\sigma, \mathbb{R}} & =\left(\mathcal{Q}_{\mathscr{P}, \mathbb{R}}\right)_{y} \\
\mathcal{Q}_{\sigma} & =\left(\mathcal{Q}_{\mathscr{P}}\right)_{y}
\end{aligned}
$$

for any $y \in \operatorname{Int}(\sigma)$, being independent of the choice of $y$.

Example 1.34. Suppose $\operatorname{dim} B=2, \sigma_{1}, \sigma_{2} \in \mathscr{P}_{\max }$ with $\sigma_{1} \cap \sigma_{2}=\tau$ a cell of dimension one, and suppose $\Delta \cap \tau=\{p\}$. Thus Int $(\tau) \backslash \Delta$ has two connected components. Then $\Lambda / \Lambda_{\mathscr{P}}$ is the constant sheaf $\mathbb{Z}$ on $\operatorname{Int}(\tau) \backslash \Delta$, and $i_{*}\left(\Lambda / \Lambda_{\mathscr{P}}\right)$ then has stalk $\mathbb{Z} \oplus \mathbb{Z}$ at $p$, which is bigger than we want. On the other hand, if $\Lambda$ has non-trivial monodromy around $p$, then $\Gamma(U \backslash\{p\}, \Lambda)=\Gamma\left(U \backslash\{p\}, \Lambda_{\mathscr{P}}\right)$ for $U$ any sufficiently small neighbourhood of $p$. Thus $i_{*} \Lambda / i_{*} \Lambda_{\mathscr{P}}$ has stalk 0 at $p$. The above definition is designed to give an intermediate value for the stalk, namely $\mathbb{Z}$.

Definition 1.35. The fan $\Sigma_{\tau}$. Suppose $B$ is integral affine with singularities and $\mathscr{P}$ is a toric polyhedral decomposition. Let $\tau \in \mathscr{P}$. We will construct a rational polyhedral fan in $\mathcal{Q}_{\tau, \mathbb{R}}$ (with integral structure $\mathcal{Q}_{\tau}$ ) associated to $\tau$. 
Let $S_{\tau}: U_{\tau} \rightarrow M_{\mathbb{R}}^{\prime}$ be as in Definition $\left.1.22,5\right)$. For any point $y \in \operatorname{Int}(\tau) \backslash \Delta, S_{\tau, *}$ identifies $\Lambda_{\mathbb{R}, y} / \Lambda_{\tau, \mathbb{R}}=\mathcal{Q}_{\tau, \mathbb{R}}$ with $M_{\mathbb{R}}^{\prime}$, so we can take $M_{\mathbb{R}}^{\prime}=\mathcal{Q}_{\tau, \mathbb{R}}$. The idea now is that if we take $U_{\tau}$ small enough, then inside $U_{\tau}$ the decomposition $\mathscr{P}$ looks like the pull back of a fan in $M_{\mathbb{R}}^{\prime}$ under the map $S_{\tau}$. Indeed, let $\mathscr{P}_{\tau}$ denote the collection of closures of connected components of the sets $\operatorname{Int}(\sigma) \cap U_{\tau}$, where $\sigma$ ranges over all elements of $\mathscr{P}$ containing $\tau$. For any $\sigma \in \mathscr{P}_{\tau}$, set

$$
K_{\sigma}=\left\{a S_{\tau}(x) \mid a \in \mathbb{R}_{\geq 0} \text { and } x \in \sigma \cap U_{\tau}\right\}=\mathbb{R}_{\geq 0} \cdot S_{\tau}(\sigma) .
$$

Because $S_{\tau}$ contracts $\operatorname{Int}(\tau)$ to a point, this can be viewed as the tangent wedge to $\sigma$ at a point $y \in \operatorname{Int}(\tau)$ modulo the linear space $\Lambda_{\tau, \mathbb{R}}$. We set

$$
\Sigma_{\tau}=\left\{K_{\sigma} \mid \sigma \in \mathscr{P}_{\tau}\right\} .
$$

It is clear that $\Sigma_{\tau}$ is a complete rational polyhedral fan in $\mathcal{Q}_{\tau, \mathbb{R}}$. Note that if $\tau$ is a vertex $v$, then this coincides with $\Sigma_{v}$ of Remark 1.24.

Note we do not necessarily have a one-to-one correspondence between cells of $\mathscr{P}$ containing $\tau$ and cones of $\Sigma_{\tau}$. This is because we have allowed the possibility that cells can be self-intersecting, as in Example 1.23, (1), where a fundamental domain for the action of $\Gamma$ on $M_{\mathbb{R}}$ is used to induce a polyhedral decomposition of $M_{\mathbb{R}} / \Gamma$. It is because of this possibility that we use the following notion to generalize the concept of inclusion of cells:

Definition 1.36. Let $\operatorname{Cat}(\mathscr{P})$ be the category whose set of objects is the set $\mathscr{P}$, and morphisms are defined as follows: if $\tau \nsubseteq \sigma$ then $\operatorname{Hom}(\tau, \sigma)=\emptyset$; if $\tau=\sigma$ then $\operatorname{Hom}(\tau, \sigma)=\{i d\}$, and if $\tau \subset \sigma$, then $\operatorname{Hom}(\tau, \sigma)$ is the set of 1 -simplices in the triangulation $\operatorname{Bar}(\mathscr{P})$ with endpoints $\operatorname{Bar}(\tau)$ and $\operatorname{Bar}(\sigma)$. If $e_{1}$ is an edge joining $\operatorname{Bar}(\tau)$ and $\operatorname{Bar}(\sigma)$ and $e_{2}$ an edge joining $\operatorname{Bar}(\sigma)$ and $\operatorname{Bar}(\omega)$, then $e_{2} \circ e_{1}$ is defined to be the third edge of the unique 2-simplex containing $e_{1}$ and $e_{2}$.

The key point here is that if the neighbourhood $U_{\tau}$ of $\operatorname{Int}(\tau)$ is taken to be sufficiently small, then there is a natural one-to-one correspondence between the set of connected components of $\operatorname{Int}(\sigma) \cap U_{\tau}$ and the set $\operatorname{Hom}(\tau, \sigma)$, with each component of $\operatorname{Int}(\sigma) \cap U_{\tau}$ intersecting precisely one element of $\operatorname{Hom}(\tau, \sigma)$. Thus there is one cone in the fan $\Sigma_{\tau}$ coming from $\sigma$ for each element of $\operatorname{Hom}(\tau, \sigma)$, and we obtain a one-to-one correspondence between cones of $\Sigma_{\tau}$ and the set

$$
\coprod_{\sigma \in \mathscr{P}} \operatorname{Hom}(\tau, \sigma) \text {. }
$$

Now we can obtain the relationship between $\Sigma_{\tau}$ and $\Sigma_{\sigma}$ when $\tau \subset$ $\sigma$; this depends on an $e \in \operatorname{Hom}(\tau, \sigma)$ corresponding to a connected component $T_{e}$ of $\operatorname{Int}(\sigma) \cap U_{\tau}$. Choose a path $\gamma$ in $U_{\tau}$ from some $x \in$ $\operatorname{Int}(\tau) \backslash \Delta$ to some $y \in T_{e} \backslash \Delta$. By parallel transport this defines an isomorphism $\Lambda_{\mathbb{R}, x} \rightarrow \Lambda_{\mathbb{R}, y}$, and hence a surjection $p_{e}: \mathcal{Q}_{\tau, \mathbb{R}} \rightarrow \mathcal{Q}_{\sigma, \mathbb{R}}$, 
which is defined independently of the path $\gamma$ by Proposition 1.32. If $K_{e}$ denotes the cone of $\Sigma_{\tau}$ corresponding to the connected component $T_{e}$, then the map $p_{e}$ is easily seen to identify $\Sigma_{\sigma}$ with the quotient fan $\Sigma_{\tau}\left(K_{e}\right)$, which is defined as follows. This identification is completely canonical, depending only on $e$.

Definition 1.37. Let $\Sigma$ be a fan on a vector space $M_{\mathbb{R}}$, and let $\tau \in \Sigma$ be a cone. We denote by $\Sigma(\tau)$ the fan in $M_{\mathbb{R}} / \mathbb{R} \tau$ consisting of the cones

$$
\{(\sigma+\mathbb{R} \tau) / \mathbb{R} \tau \mid \sigma \supseteq \tau, \sigma \in \Sigma\} .
$$

We say $\Sigma(\tau)$ is the quotient fan of $\Sigma$ by the cone $\tau$.

We also denote by $\tau^{-1} \Sigma$ the fan of (not strictly convex!) cones

$$
\{\sigma+\mathbb{R} \tau \mid \sigma \supseteq \tau, \sigma \in \Sigma\} .
$$

We call this the localisation of the fan $\Sigma$ at $\tau$.

Definition 1.38. The normal fan $\check{\Sigma}_{\tau}$. Let $\tau \in \mathscr{P}$, and let $y \in$ $\operatorname{Int}(\tau) \backslash \Delta$ be any point. Let $v$ be a vertex of $\tau$ contained in the same connected component of $\tau \backslash \Delta$ as $y$, and let $R_{v}, \mathscr{P}_{v}$, and $\exp _{v}$ be as usual, and $\tilde{\tau} \in \mathscr{P}_{v}$ with $0 \in \tilde{\tau}$ and $\exp _{v}(\tilde{\tau})=\tau$. Then via parallel translation along $\tau, \Lambda_{\mathbb{R}, v}$ can be canonically identified with $\Lambda_{\mathbb{R}, y}$ so that $\mathbb{R} \tilde{\tau}$ and $\Lambda_{\tau, \mathbb{R}}$ are identified. We can then view $\tilde{\tau} \subseteq \Lambda_{\tau, \mathbb{R}}$ as a polytope under this identification, with $\operatorname{dim} \tilde{\tau}=\operatorname{dim} \Lambda_{\tau, \mathbb{R}}$. In particular, $\tilde{\tau}$ is well-defined up to translation in $\Lambda_{\tau, \mathbb{R}}$ independently of the choice of $y$. This associates to each cell a canonical (up to translation) polytope.

We define $\check{\Sigma}_{\tau}$ to be the normal fan in $\Lambda_{\tau, \mathbb{R}}^{*}$ to $\tilde{\tau}$ in the usual way, as follows. For each face $\tilde{\omega} \subseteq \tilde{\tau}$, let

$$
\check{K}_{\omega}=\left\{f \in \Lambda_{\tau, \mathbb{R}}^{*}|f|_{\tilde{\omega}} \text { is constant and }\langle f, z\rangle \geq\langle f, x\rangle \text { for } z \in \tilde{\tau}, x \in \tilde{\omega}\right\} .
$$

Then the collection of cones $\left\{\check{K}_{\omega}\right\}$ forms the normal fan $\check{\Sigma}_{\tau}$ in $\Lambda_{\tau, \mathbb{R}}^{*}$, which is completely well-defined. It is then easy to see that there is a one-to-one correspondence between cones of $\check{\Sigma}_{\tau}$ and the set

$$
\coprod_{\omega \in \mathscr{P}} \operatorname{Hom}(\omega, \tau) \text {. }
$$

Definition 1.39. Let $\mathscr{P}$ be a polyhedral decomposition of $B$. Let $\mathcal{A} f_{\mathbb{R}}(B, \mathbb{R})$ denote the sheaf of continuous functions on $B$ to $\mathbb{R}$ which are affine when restricted to $B_{0}$. We define $\mathcal{A} f f(B, \mathbb{Z})$ to be the subsheaf of $\mathcal{A f f}_{\mathbb{R}}(B, \mathbb{R})$ of functions which when restricted to $B_{0}$ are in $\mathcal{A} f f\left(B_{0}, \mathbb{Z}\right)$ (Definition 1.11).

Global affine functions are either unbounded or constant.

Proposition 1.40. Let $\mathscr{P}$ be a polyhedral decomposition of $B$ and $\varphi: B \rightarrow \mathbb{R}$ a bounded integral affine function. Then $\varphi$ is constant. 
Proof. Assume $\varphi$ is non-constant. Then its linear part is non-zero at every $x \in B_{0}$. We construct a piecewise integral affine map $\gamma: \mathbb{R}_{\geq 0} \rightarrow B$ with intervals of linearity mapping to edges of $\mathscr{P}$ and such that $\varphi \circ \gamma$ is strictly monotone. Then $\varphi \circ \gamma$ is unbounded because $\gamma$ traces through infinitely many edges and on each edge $\varphi \circ \gamma$ increases at least by 1 .

First note that for each edge $\tau \in \mathscr{P}$ the restriction $\left.\varphi\right|_{\operatorname{Int}(\tau)}$ is affine, regardless of $\tau$ intersecting $\Delta$ or not. In fact, there exists a top-dimensional cell $\sigma \in \mathscr{P}$ containing $\tau$ and $\left.\varphi\right|_{\operatorname{Int}(\sigma)}$ is affine linear. By continuity of $\varphi$ the pull-back to some polytope $\tilde{\sigma}$ covering $\sigma$ is then also affine, and there is an edge $\tilde{\tau} \subset \tilde{\sigma}$ mapping to $\tau$.

Now we construct $\gamma$ inductively; in the $n$-th step $\gamma_{n}$ passes through $n$ edges. For $n=0$ just choose a vertex $v \in B$ and let $\gamma_{0}:\{0\} \rightarrow B$ have image $v$. To construct $\gamma_{n+1}$ let $\tau$ be the last edge passed through by $\gamma_{n}$. Thus if $\gamma_{n}$ is defined on $[0, a]$ and the integral length of $\tau$ is $l$ then $\left.\gamma_{n}\right|_{[a-l, a]}$ is given by $[a-l, a] \simeq \tilde{\tau} \rightarrow B$. The identification of $[a-l, a]$ with $\tilde{\tau}$ is done in such a way that $a-l$ maps to $\gamma_{n}(a-l)=$ $\gamma_{n-1}(a-l)$. At the endpoint $w=\gamma_{n}(a)$ the level set of $\varphi$ locally separates $B$ into two connected components with $\gamma_{n}([a-\epsilon, a))$ being contained in only one of them for $\epsilon$ small. Now because $\mathscr{P}$ defines a fan structure at $w$ there exists an edge $\tau^{\prime} \in \mathscr{P}$ emanating from $w$ into the other connected component. Then $\left.\varphi\right|_{\tau^{\prime}}$ is strictly increasing when starting from $w$. Hence we can attach an interval equal to the integral length of $\tau^{\prime}$ to the domain of definition of $\gamma_{n}$ and map it in an integral affine way onto $\tau^{\prime}$ to obtain $\gamma_{n+1}$.

q.e.d.

Proposition 1.41. If $i: B_{0} \hookrightarrow B$ denotes the inclusion and $B$ has a polyhedral decomposition $\mathscr{P}$, then $\mathcal{A} f f_{\mathbb{R}}(B, \mathbb{R})=i_{*} \mathcal{A} f f_{\mathbb{R}}\left(B_{0}, \mathbb{R}\right)$ and $\mathcal{A} f f(B, \mathbb{Z})=i_{*} \mathcal{A} f f\left(B_{0}, \mathbb{Z}\right)$.

Proof. Let $U \subseteq B$ be open, $U_{0}=U \backslash \Delta$. Then there is certainly an inclusion given by restriction $\mathcal{A f f}_{\mathbb{R}}(U, \mathbb{R}) \rightarrow \mathcal{A} f f_{\mathbb{R}}\left(U_{0}, \mathbb{R}\right)$, because $U_{0}$ is dense in $U$ and thus a function on $U_{0}$ has at most one continuous extension to $U$. Conversely, given an affine function on $U_{0}$, to extend it to $U$ we need only to find extensions on an open covering of $U$; by uniqueness these extensions will glue. Thus we can assume $U$ to be as small as we like. In particular, we can assume $U$ is a small neighbourhood of a point $y \in \operatorname{Int}(\tau) \cap \Delta, \tau \in \mathscr{P}$. Let $v$ be a vertex of $\tau, R_{v}, \mathscr{P}_{v}$, $\exp _{v}$ as usual, $\tilde{\tau} \in \mathscr{P}_{v}$ with $0 \in \tilde{\tau}$ and $\exp _{v}(\tilde{\tau})=\tau$. Let $\tilde{y} \in \operatorname{Int}(\tilde{\tau}) \operatorname{satisfy}_{\exp _{v}}(\tilde{y})=y$, and let $\tilde{U}$ be a lift of $U$ to an open neighbourhood of $\tilde{y}$. If $\tilde{\sigma}_{1}, \ldots, \tilde{\sigma}_{m}$ are the maximal cells of $\mathscr{P}_{v}$ containing $\tilde{\tau}$, then

$$
f_{i}:=\left.f \circ \exp _{v}\right|_{\left(\tilde{U} \cap \tilde{\sigma}_{i}\right) \backslash \exp _{v}^{-1}(\Delta)}
$$

extends to an affine map $\psi_{i}: \tilde{U} \cap \tilde{\sigma}_{i} \subseteq \Lambda_{\mathbb{R}, v} \rightarrow \mathbb{R}$. Furthermore, because $f_{i}$ and $f_{j}$ agree on $\left(\tilde{U} \cap \tilde{\sigma}_{i} \cap \tilde{\sigma}_{j}\right) \backslash \exp _{v}^{-1}(\Delta)$, by continuity, $\psi_{i}$ and $\psi_{j}$ agree on $\tilde{\sigma}_{i} \cap \tilde{\sigma}_{j}$. Thus the $\psi_{i}$ 's glue to give a function $\psi: \tilde{U} \rightarrow \mathbb{R}$. 
It follows that $f$ can be extended across $\Delta$ as $\left.\psi \circ \exp _{v}^{-1}\right|_{U}$. The same argument works in the integral case.

q.e.d.

Proposition 1.42. If $B$ has a polyhedral decomposition, then there are exact sequences

$$
0 \longrightarrow \mathbb{R} \longrightarrow \mathcal{A} f f_{\mathbb{R}}(B, \mathbb{R}) \longrightarrow i_{*} \check{\Lambda}_{\mathbb{R}} \longrightarrow 0
$$

and

$$
0 \longrightarrow \mathbb{Z} \longrightarrow \mathcal{A f f}(B, \mathbb{Z}) \longrightarrow i_{*} \check{\Lambda} \longrightarrow 0 .
$$

Proof. We have from Definition 1.11 an exact sequence

$$
0 \longrightarrow \mathbb{R} \longrightarrow \mathcal{A} f f_{\mathbb{R}}\left(B_{0}, \mathbb{R}\right) \longrightarrow \check{\Lambda}_{\mathbb{R}} \longrightarrow 0
$$

on $B_{0}$, hence an exact sequence

$$
0 \longrightarrow \mathbb{R} \longrightarrow \mathcal{A} f f_{\mathbb{R}}(B, \mathbb{R}) \longrightarrow i_{*} \check{\Lambda}_{\mathbb{R}}
$$

by Proposition 1.41, so we just need to show surjectivity on the right. By Proposition 1.12 and Proposition 1.29, there is an open cover $\left\{U_{\tau} \mid \tau \in\right.$ $\mathscr{P}\}$ of $B$ such that the exact sequence for $\mathcal{A} f f\left(B_{0}, \mathbb{R}\right)$ splits on $U_{\tau} \cap B_{0}$ for each $\tau \in \mathscr{P}$. But then on $U_{\tau}, \mathcal{A f f}(B, \mathbb{R})=\mathbb{R} \oplus i_{*} \check{\Lambda}_{\mathbb{R}}$, so we have surjectivity.

q.e.d.

Next we wish to define piecewise linear functions. One natural definition would be to define a piecewise linear function on $B$ with respect to a polyhedral decomposition $\mathscr{P}$ as a continuous function on $B$ which is affine on the interior of every maximal simplex. However, this only depends on the affine structure of the polytopes and ignores singularities, and this proposed definition can behave a bit perversely near singularities. Instead, we refine this by

Definition 1.43. Let $\mathscr{P}$ be a polyhedral decomposition of $B$. If $U \subseteq$ $B$ is an open set, then a piecewise linear function on $U$ is a continuous function $\varphi: U \rightarrow \mathbb{R}$ which is affine on $U \cap \operatorname{Int}(\sigma)$ for each $\sigma \in \mathscr{P}_{\max }$, and satisfies the following property: for any $y \in U, y \in \operatorname{Int}(\sigma)$ for some $\sigma \in \mathscr{P}$, there exists a neighbourhood $V$ of $y$ and a $f \in \Gamma\left(V, \mathcal{A} f f_{\mathbb{R}}(B, \mathbb{R})\right)$ such that $\varphi-f$ is zero on $V \cap \operatorname{Int}(\sigma)$. We denote by $\mathcal{P} \mathcal{L}_{\mathscr{P}, \mathbb{R}}(B, \mathbb{R})$ the sheaf on $B$ of such functions. Similarly, we define the subsheaf of integral piecewise linear functions $\mathcal{P} \mathcal{L}_{\mathscr{P}}(B, \mathbb{Z}) \subseteq \mathcal{P} \mathcal{L}_{\mathscr{P}, \mathbb{R}}(B, \mathbb{R})$ so that $\varphi \in \mathcal{P} \mathcal{L}_{\mathscr{P}}(B, \mathbb{R})$ if $\left.\varphi\right|_{\operatorname{Int}(\sigma)} \in \mathcal{A} f f(\operatorname{Int}(\sigma), \mathbb{Z})$ for each $\sigma \in \mathscr{P}_{\max }$, and $f$ can be taken to be integral in a neighbourhood of each $y$.

The restriction on the behaviour near the singularities of the affine structure makes the following definition possible.

Definition 1.44. Let $\mathscr{P}$ be a toric polyhedral decomposition of $B$ and $\varphi$ be a piecewise linear function on $B$. For each $\sigma \in \mathscr{P}$, we obtain by Definition 1.35 a fan $\Sigma_{\sigma}$ in $\mathcal{Q}_{\sigma, \mathbb{R}}$. Choose any point $y \in \operatorname{Int}(\sigma)$, and a small open neighbourhood $U$ of $y$. By Definition 1.43 there exists 
an affine function $f: U \rightarrow \mathbb{R}$ such that $\left.f\right|_{U \cap \operatorname{Int}(\sigma)}=\left.\varphi\right|_{U \cap \operatorname{Int}(\sigma)}$. Then $\varphi-f$ is the pullback by $S_{\sigma}: U \rightarrow \mathcal{Q}_{\sigma, \mathbb{R}}$ of a function on $\mathcal{Q}_{\sigma, \mathbb{R}}$ which is piecewise linear with respect to the fan $\Sigma_{\sigma}$. We write this function as $\varphi_{\sigma}$, well-defined up to a linear function.

Definition 1.45. If $\mathscr{P}$ is a polyhedral decomposition of $B$, then there is a commutative diagram of exact sequences

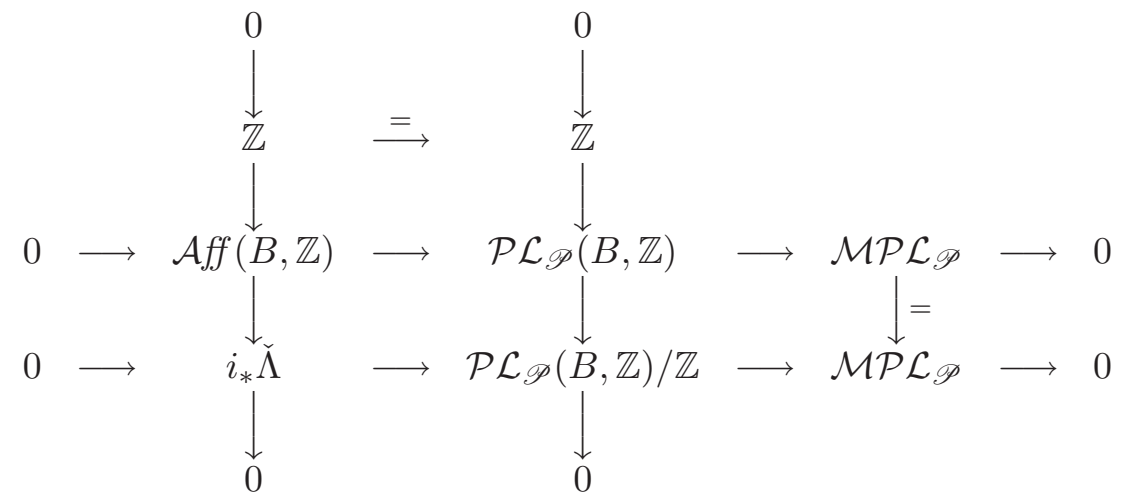

defining a sheaf $\mathcal{M P} \mathcal{P} \mathscr{P}$. A similar diagram with $\mathbb{R}$ subscripts defines a sheaf $\mathcal{M P} \mathcal{L}_{\mathscr{P}, \mathbb{R}}$.

Definition 1.46. A multi-valued piecewise linear function on an open subset $U \subseteq B$ with respect to $\mathscr{P}$ is an element $\varphi \in \Gamma\left(U, \mathcal{M P} \mathcal{L}_{\mathscr{P}, \mathbb{R}}\right)$; it is integral if it is in $\Gamma\left(U, \mathcal{M P} \mathcal{L}_{\mathscr{P}}\right)$. Such a section should be thought of as a collection of piecewise linear functions on an open covering $U_{i}$ of $U$ which differ only by affine linear functions on overlaps. The first Chern class of $\varphi \in H^{0}\left(B, \mathcal{M P} \mathcal{L}_{\mathscr{P}, \mathbb{R}}\right)$ is the image of $\varphi$ under the boundary map

$$
c_{1}: H^{0}\left(B, \mathcal{M P} \mathcal{L}_{\mathscr{P}, \mathbb{R}}\right) \longrightarrow H^{1}\left(B, i_{*} \check{\Lambda}_{\mathbb{R}}\right)
$$

(or $c_{1}: H^{0}\left(B, \mathcal{M P} \mathcal{L}_{\mathscr{P}}\right) \rightarrow H^{1}\left(B, i_{*} \check{\Lambda}\right)$ in the integral case). Using Definition 1.44, a multi-valued piecewise linear function on $B$ determines a piecewise linear function $\varphi_{\sigma}: \mathcal{Q}_{\sigma, \mathbb{R}} \rightarrow \mathbb{R}$ with respect to $\Sigma_{\sigma}$, welldefined up to a linear function.

Definition 1.47. A multi-valued piecewise linear function $\varphi$ on $B$ with respect to $\mathscr{P}$ is (strictly) convex if $\varphi_{\tau}$ is a (strictly) convex piecewise linear function on the fan $\Sigma_{\tau}$ for all $\tau \in \mathscr{P}$. More precisely, if $\sigma$ is a maximal cone of $\Sigma_{\tau}$ and $n_{\sigma} \in \mathcal{Q}_{\tau, \mathbb{R}}^{*}$ satisfies $\left\langle n_{\sigma}, y\right\rangle=\varphi_{\tau}(y)$ for all $y \in \sigma$, then $\left\langle n_{\sigma}, y\right\rangle \leq \varphi_{\tau}(y)\left(\left\langle n_{\sigma}, y\right\rangle<\varphi_{\tau}(y)\right)$ for all $y \notin \sigma$. Note this is independent of the choice of $\varphi_{\tau}$.

While we will not use it in this paper, the following concept is a natural generalisation of regular triangulations:

Definition 1.48. A polyhedral decomposition $\mathscr{P}$ is regular if there is a strictly convex multi-valued piecewise linear function on $B$ with respect to $\mathscr{P}$. 
One of the key points of this paper is that strictly convex piecewise linear functions can play the same role as the affine Kähler potentials discussed in §1: in other words, they allow us to dualize the affine structure using a discrete Legendre transform which we will explain in the next section.

Remark 1.49. Straightening the discriminant locus. When we construct an affine manifold with singularities using Construction 1.26, the discriminant locus has been chosen to be a union of simplices in the barycentric subdivision of $\mathscr{P}$. The intersection and dual intersection complexes we construct in $\S 4$ are special cases of Construction 1.26. However, in real life, one might encounter affine manifolds with singularities arising from other sources, such as elliptically fibred K3 surfaces (Example 1.17). Often these examples carry polyhedral decompositions, but there is no reason to expect the discriminant locus to be a union of barycentric simplices. It is convenient to note these affine manifolds can then be deformed into examples of the sort constructed in Construction 1.26 .

Thus if $B, \mathscr{P}$ does not arise through Construction 1.26, it will often make sense to replace $B$ and $\mathscr{P}$ with a closely related $B_{\text {str }}$ and $\mathscr{P}_{\text {str }}$ as follows. Each maximal cell $\sigma$ of $\mathscr{P}$ determines a lattice polytope $\tilde{\sigma} \subseteq \Lambda_{\mathbb{R}, y}$ for any $y \in \operatorname{Int}(\sigma)$ using Definition 1.38. Furthermore, $B$ is clearly obtained as a topological space via integral affine identifications between various faces of the polytopes of

$$
\mathscr{P}^{\prime}=\left\{\tilde{\sigma} \mid \sigma \in \mathscr{P}_{\max }\right\} .
$$

Also, we have a fan structure $\Sigma_{v}$ at each vertex of $\mathscr{P}$, and by Construction 1.26 and Proposition 1.27, we obtain a new affine manifold with singularities structure on $B$, which we call $B_{\text {str }}$, with discriminant locus $\Delta_{s t r}$ contained in $\Delta_{s t r}^{\prime}$, the union of all codimension two simplices of $\operatorname{Bar}(\mathscr{P})$ not containing vertices or intersecting interiors of maximal cells of $\mathscr{P}$. Then as a manifold $B_{s t r}=B$, and $\mathscr{P}_{\text {str }}=\mathscr{P}$, but the structure of affine manifolds with singularities is different because $\Delta_{\text {str }} \neq \Delta$. In the following figure, we see the effects of straightening. The dotted lines show the first barycentric subdivision.
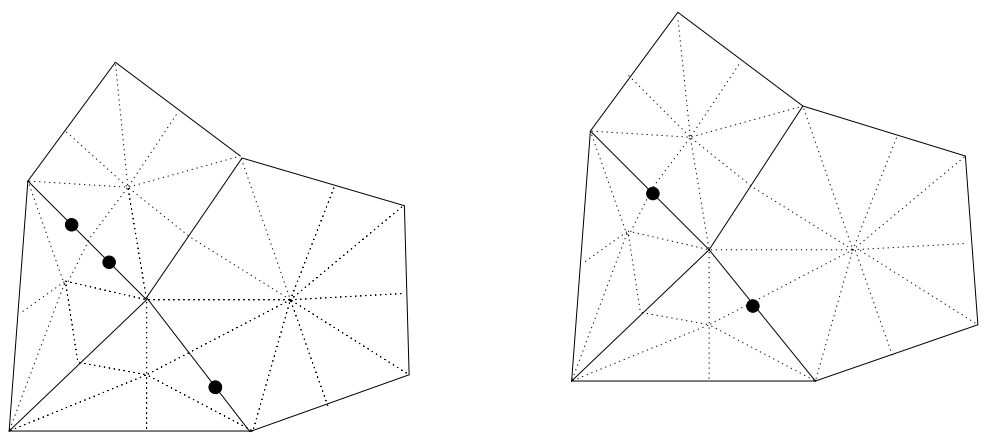
All of our examples of affine manifolds with singularities will essentially arise through Construction 1.26 , so this straightening will not be necessary in this paper.

1.4. The discrete Legendre transform. We now introduce a key concept for relating an algebro-geometric form of mirror symmetry to the Strominger-Yau-Zaslow approach. Let $B$ be an integral affine manifold with singularities with a toric polyhedral decomposition $\mathscr{P}$, and assume the discriminant locus of $B$ has been straightened, or equivalently, $(B, \mathscr{P})$ arises from Construction 1.26 . Let $\varphi$ be a strictly convex multi-valued integral piecewise linear function on $B$. We will construct a new integral affine manifold with singularities $\check{B}$ with discriminant locus $\check{\Delta}$. As manifolds, $B=\check{B}$ and $\Delta=\check{\Delta}$, but the affine structure is dual. In addition, we obtain a toric polyhedral decomposition $\check{\mathscr{P}}$ and a strictly convex multi-valued integral piecewise linear function $\check{\varphi}$ on $\check{B}$. We will say $(\check{B}, \check{\mathscr{P}}, \check{\varphi})$ is the discrete Legendre transform of $(B, \mathscr{P}, \varphi)$.

First we define $\check{\mathscr{P}}$. For any $\sigma \in \mathscr{P}$, define $\check{\sigma}$ to be the union of all simplices in $\operatorname{Bar}(\mathscr{P})$ intersecting $\sigma$ but disjoint from any proper subcell of $\sigma$. Put

$$
\check{\mathscr{P}}=\{\check{\sigma} \mid \sigma \in \mathscr{P}\} .
$$

This is the usual dual cell to $\sigma$, with $\operatorname{dim} \check{\sigma}=n-\operatorname{dim} \sigma$. The figure below shows what $\check{\mathscr{P}}$ looks like for the example of Remark 1.49.

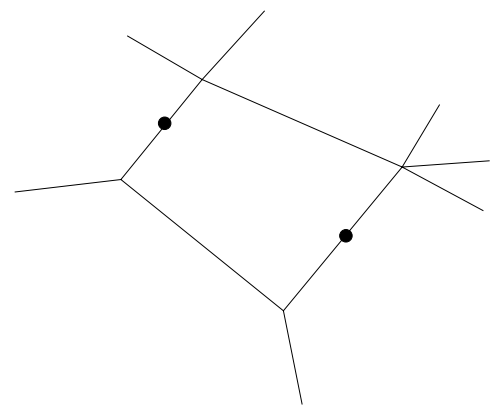

Of course, $\check{\sigma}$ is usually not a polyhedron with respect to the affine structure on $B$, and we will build a new affine structure using Construction 1.26 .

For any $\sigma \in \mathscr{P}$, we obtain a fan $\Sigma_{\sigma}$ living in $\mathcal{Q}_{\sigma, \mathbb{R}}$ and a piecewise linear function $\varphi_{\sigma}$ on $\Sigma_{\sigma}$ by Definition 1.44, defined up to choice of a linear function. This function is strictly convex by assumption, and we can consider the corresponding Newton polytope, i.e., set

$$
\widetilde{\sigma}=\left\{x \in \mathcal{Q}_{\sigma, \mathbb{R}}^{*} \mid\langle x, y\rangle \geq-\varphi_{\sigma}(y) \quad \forall y \in \mathcal{Q}_{\sigma, \mathbb{R}}\right\} .
$$

Note that because $\varphi_{\sigma}$ is strictly convex there is a one-to-one inclusion reversing correspondence between the faces of $\widetilde{\sigma}$ and cones in $\Sigma_{\sigma}$. Given $e \in \operatorname{Hom}(\sigma, \tau)$ for some $\tau \in \mathscr{P}$, there is a corresponding cone $\tau_{e} \in \Sigma_{\sigma}$, 
with corresponding cell $\check{\tau}_{e} \subseteq \widetilde{\sigma}$ given by

$$
\check{\tau}_{e}=\left\{x \in \widetilde{\sigma} \mid\langle x, y\rangle=-\varphi_{\sigma}(y) \quad \forall y \in \tau_{e}\right\} .
$$

In addition, $\widetilde{\sigma}$ is an integral polytope because $\varphi_{\sigma}$ has integral slopes.

We can glue these polyhedra together as follows. In the category $\underline{\operatorname{Cat}}(\mathscr{P})$, let $e \in \operatorname{Hom}(\sigma, \tau)$, determining a face $\check{\tau}_{e}$ of $\widetilde{\sigma}$. In addition, because $\Sigma_{\tau}=\Sigma_{\sigma}\left(\tau_{e}\right)$, it is easy to see that $\widetilde{\widetilde{\tau}} \subseteq \mathcal{Q}_{\tau, \mathbb{R}}^{*} \subseteq \mathcal{Q}_{\sigma, \mathbb{R}}^{*}$ is just a translate of the face $\check{\tau}_{e}$ of $\widetilde{\sigma}$. This yields a contravariant functor $F$ from $\underline{\operatorname{Cat}}(\mathscr{P})$ to the category of topological spaces with $F(\tau)=\widetilde{\widetilde{\tau}}$. For $e \in \operatorname{Hom}(\sigma, \tau), F(e): \widetilde{\tau} \rightarrow \widetilde{\sigma}$ is the unique identification via translation of $\widetilde{\tau}$ with the face $\check{\tau}_{e}$ of $\widetilde{\sigma}$. We can then construct the limit of $F$, i.e., the quotient space of $\coprod_{\sigma \in \mathscr{P}} \widetilde{\widetilde{\sigma}}$ by these identifications. We will write this quotient map as

$$
\check{\pi}: \coprod_{\sigma \in \mathscr{P}} \widetilde{\sigma} \longrightarrow \check{B}
$$

It is easy to see this quotient space is homeomorphic to $B$ itself, with the image of $\widetilde{\sigma}$ in $B$ being $\check{\sigma}$. This is just a combinatorial identification, but we can achieve this as a $C^{0}$ map by mapping $\widetilde{\sigma}$ to $\check{\sigma} \subseteq B$ in a piecewise linear way on the barycentric subdivisions. In particular, we have now constructed $\check{B}$ by identifying via affine transformations the faces of the lattice polytopes $\widetilde{\sigma}$ where $\sigma$ runs over minimal cells of $\mathscr{P}$. This is the first step in constructing an affine manifold via the method of Construction 1.26. Note that under this piecewise linear identification of $B$ and $\check{B}$, we have

$$
\operatorname{Bar}(\check{\mathscr{P}})=\operatorname{Bar}(\mathscr{P})
$$

and

$$
\underline{\operatorname{Cat}}(\check{\mathscr{P}})=\underline{\operatorname{Cat}}(\mathscr{P})^{\text {op }} .
$$

We also take the discriminant locus $\check{\Delta}^{\prime}$ as usual as the union of codimension two simplices not intersecting vertices of $\check{\mathscr{P}}$ or interiors of maximal cells of $\check{\mathscr{P}}$. Of course $\Delta \subseteq \check{\Delta}^{\prime}$. The latter discriminant locus is only provisional, and we will shrink it in Proposition 1.50 below.

To finish specifying an integral affine structure on $\check{B} \backslash \check{\Delta}^{\prime}$, we just need to specify a fan structure at each vertex $\check{\sigma}$ of $\check{\mathscr{P}}$ (for $\sigma$ a maximal cell of $\mathscr{P})$.

Let $y$ be the barycenter of a maximal cell $\sigma$, so $\check{\sigma}=\{y\}$. The cones of the desired fan $\Sigma_{\check{\sigma}}$ should be in one-to-one correspondence with

$$
\coprod_{\check{\tau} \in \check{\mathscr{P}}} \operatorname{Hom}(\check{\sigma}, \check{\tau})=\coprod_{\tau \in \mathscr{P}} \operatorname{Hom}(\tau, \sigma),
$$

and the latter set is in one-to-one correspondence with cones in the normal fan $\check{\Sigma}_{\sigma}$ (see Definition 1.38). So it is enough to give integral affine identifications as follows. Let $e \in \operatorname{Hom}(\tau, \sigma)$, so $y$ is an endpoint of $e$ and the other endpoint of $e$ is the barycenter of $\tau$. Then by Definition 1.38, 
we obtain $\tilde{\tau} \subseteq \tilde{\sigma} \subseteq \Lambda_{\mathbb{R}, y}$, well-defined up to translation, and thus we can assume $0 \in \tilde{\tau}$, getting the corresponding cone of $\check{\Sigma}_{\sigma}$ being

$$
\begin{aligned}
\check{K}_{e} & =\left\{f \in \Lambda_{\mathbb{R}, y}^{*}|f|_{\tilde{\tau}} \text { is constant and }\langle f, z\rangle \geq\langle f, x\rangle \text { for all } z \in \tilde{\sigma}, x \in \tilde{\tau}\right\} \\
& =\left\{f \in(\mathbb{R} \tilde{\tau})^{\perp} \mid\langle f, z\rangle \geq 0 \quad \forall z \in \tilde{\sigma}\right\} .
\end{aligned}
$$

On the other hand, the choice of $e$ selects a maximal cone $\sigma_{e} \in \Sigma_{\tau}$ and hence a vertex $\check{\sigma}_{e}$ of $\widetilde{\widetilde{\tau}}$, and $\widetilde{\tau}$ has tangent wedge at this vertex given by the dual cone to $\sigma_{e}$, namely

$$
\left\{f \in \mathcal{Q}_{\tau, \mathbb{R}}^{*} \mid\langle f, z\rangle \geq 0 \quad \forall z \in \sigma_{e} \subseteq \mathcal{Q}_{\tau, \mathbb{R}}\right\} .
$$

We must compare these two cones as follows. We can deform $e$ slightly to a path $\gamma$ connecting $y \in \operatorname{Int}(\sigma)$ to $y^{\prime} \in \operatorname{Int}(\tau) \backslash \Delta$; parallel transport along $\gamma$ identifies $\Lambda_{\mathbb{R}, y}$ and $\Lambda_{\mathbb{R}, y^{\prime}}$ in such a way that $\mathbb{R} \tilde{\tau}$ and $\left(\Lambda_{\mathscr{P}, \mathbb{R}}\right)_{y^{\prime}}$ are identified. Under this identification, $(\mathbb{R} \tilde{\tau})^{\perp}$ is identified with $\mathcal{Q}_{\tau, \mathbb{R}}^{*}$, and it is then clear the two cones defined above coincide. This gives the integral affine transformation identifying $\check{K}_{e}$ with the corresponding tangent wedge of $\widetilde{\tau}$. This is sufficient to define the fan structure of $\check{B}$ at $y$, and thus we obtain an integral affine structure on $\check{B} \backslash \check{\Delta}^{\prime}$.

Proposition 1.50. Let $B$ be an integral affine manifold with singularities with a toric polyhedral decomposition $\mathscr{P}$, and a strictly convex multi-valued integral piecewise linear function $\varphi$ on $B$. Let $\check{B}$ be as constructed above. Then

1) Under the canonical identification $\check{B}=B$ the linear part of the holonomy representation $\rho^{\check{B}}: \pi_{1}\left(\check{B} \backslash \check{\Delta}^{\prime}\right) \rightarrow \operatorname{Aff}\left(N_{\mathbb{R}}\right)$ is dual to the linear part of the holonomy representation $\rho^{B}: \pi_{1}\left(B \backslash \Delta^{\prime}\right) \rightarrow$ $\operatorname{Aff}\left(M_{\mathbb{R}}\right)$ (where $\left.\Delta^{\prime}:=\check{\Delta}^{\prime}\right)$. In other words,

$$
\tilde{\rho}^{\check{B}}(\gamma)={ }^{t} \tilde{\rho}^{B}\left(\gamma^{-1}\right)
$$

for all $\gamma \in \pi_{1}\left(B \backslash \Delta^{\prime}\right)$. Thus in particular, by Proposition 1.27, the affine structure on $\check{B} \backslash \check{\Delta}^{\prime}$ extends to $\check{B}_{0}=\check{B} \backslash \check{\Delta}$, where $\check{\Delta}=\Delta$. In addition,

$$
\Lambda^{B_{0}} \cong \check{\Lambda}^{\check{B}_{0}}
$$

and

$$
\check{\Lambda}^{B_{0}} \cong \Lambda^{\check{B}_{0}}
$$

where the superscripts denote the affine structure with respect to which these local systems are defined. (These are isomorphisms of abstract sheaves, not as subsheaves of the tangent or cotangent bundles, as the identification $B_{0}=\check{B}_{0}$ is not differentiable.)

2) $\check{\mathscr{P}}$ is a toric polyhedral decomposition of $\check{B}$.

3) The radiance obstruction of $\check{B}_{0}$ coincides with $c_{1}(\varphi) \in H^{1}\left(B, i_{*} \check{\Lambda}^{B_{0}}\right)$ under a natural inclusion

$$
H^{1}\left(B, i_{*} \check{\Lambda}^{B_{0}}\right) \hookrightarrow H^{1}\left(\check{B}_{0}, \Lambda^{\check{B}_{0}}\right) .
$$


4) For $\sigma \in \mathscr{P}, \check{\Sigma}_{\sigma}$ and $\Sigma_{\check{\sigma}}$ coincide, and $\check{\Sigma}_{\check{\sigma}}$ and $\Sigma_{\sigma}$ coincide.

Proof.

1) A path $\gamma$ in $\check{B} \backslash \check{\Delta}^{\prime}$ passes successively through open $\operatorname{sets} \operatorname{Int}\left(\check{v}_{1}\right)$ to $W_{\check{\sigma}_{1}}$ to $\operatorname{Int}\left(\check{v}_{2}\right)$ to $W_{\check{\sigma}_{2}}$ etc., where $v_{1}, \ldots, v_{n}$ are vertices of $\mathscr{P}$ and $\sigma_{1}, \ldots, \sigma_{n}$ are maximal cells of $\mathscr{P}$; here $W_{\check{\sigma}_{i}}$ is the open neighbourhood of the vertex $\check{\sigma}_{i}$ given in Definition 1.25, and in fact $W_{\check{\sigma}_{i}}=\operatorname{Int}\left(\sigma_{i}\right)$ and $\operatorname{Int}\left(\check{v}_{i}\right)=W_{v_{i}}$. Consider parallel transport from $\operatorname{Int}\left(\check{v}_{i}\right)$ to $W_{\check{\sigma}_{i}}$ in the local system $\Lambda_{\mathbb{R}}^{\check{B}_{0}}$ given by the affine structure on $\check{B}_{0}$. Now $v_{i}$ is the barycenter of $\operatorname{Int}\left(\check{v}_{i}\right)$ and $\check{\sigma}_{i}=\left\{y_{i}\right\}$, with $y_{i}$ the barycenter of $\sigma_{i}$. Then by construction, $\Lambda_{\mathbb{R}, v_{i}}^{\check{B}_{0}}$ is identified with $\mathcal{Q}_{v_{i}, \mathbb{R}}^{*}=\check{\Lambda}_{\mathbb{R}, v_{i}}$ and $\Lambda_{\mathbb{R}, y_{i}}^{\check{B}_{0}}$ is identified with $\check{\Lambda}_{\mathbb{R}, y_{i}}$. Furthermore, according to the description of the fan structure above, $\check{\Lambda}_{\mathbb{R}, v_{i}}$ and $\check{\Lambda}_{\mathbb{R}, y_{i}}$ are identified using parallel translation in $\Lambda_{\mathbb{R}}$ between $v_{i}$ and $y_{i}$. Thus parallel translation in $\Lambda_{\mathbb{R}}^{\check{B}_{0}}$ from $v_{i}$ to $y_{i}$ is the inverse transpose of parallel translation in $\Lambda_{\mathbb{R}}$ from $v_{i}$ to $y_{i}$. This makes it clear that $\tilde{\rho}^{\check{B}}(\gamma)={ }^{t} \tilde{\rho}^{B}\left(\gamma^{-1}\right)$ for $\gamma$ a loop.

In particular, the linear part of the holonomy about a codimension two simplex of $\breve{\Delta}^{\prime}$ is trivial if and only if the linear part of the holonomy of $B$ is trivial around the simplex. In addition, by Proposition 1.29, the local radiance obstruction near the simplex vanishes, so if the linear part of the holonomy is trivial, so is the holonomy itself. Hence by Proposition 1.27 we can take $\check{\Delta}=\Delta$. In addition, the identifications of local systems follow from the statement about $\tilde{\rho}^{B}$ and $\tilde{\rho}^{\check{B}}$.

2) Since we constructed $\check{B}$ and $\check{\mathscr{P}}$ by means of Construction 1.26 , $\check{\mathscr{P}}$ is a polyhedral decomposition. That it is toric follows from (1) and Proposition 1.32. Indeed, if $y \in \operatorname{Int}(\check{\tau}) \backslash \check{\Delta}$, it is enough to show that for any simple loop based at $y$ around a codimension two simplex $\omega$ of $\check{\Delta}$ intersecting $\operatorname{Int}(\check{\tau})$,

$$
\left(\tilde{\rho}^{\check{B}}(\gamma)-I\right)\left(\Lambda_{\mathbb{R}, y}^{\check{B}_{0}}\right) \subseteq\left(\Lambda_{\check{\mathscr{P}}, \mathbb{R}}^{\check{B}_{0}}\right)_{y}=\Lambda_{\check{\tau}, \mathbb{R}}^{\check{B}_{0}}
$$

But $\Lambda_{\mathbb{R}, y}^{\check{B}_{0}}$ can be identified with $\check{\Lambda}_{\mathbb{R}, y}$, and $\Lambda_{\check{\tau}, \mathbb{R}}^{\check{B}_{0}}$ can be identified with $\mathcal{Q}_{\tau, \mathbb{R}}^{*}$. Also $\tilde{\rho}^{\check{B}}(\gamma)={ }^{t} \tilde{\rho}^{B}\left(\gamma^{-1}\right)$. Now $\omega$ contains the barycenter of $\check{\tau}$, which is also the barycenter of $\tau$, so by Proposition 1.29,

$$
\left(\tilde{\rho}^{B}\left(\gamma^{-1}\right)-I\right)\left(\left(\Lambda_{\mathscr{P}, \mathbb{R}}\right)_{y}\right)=0,
$$

from which it immediately follows that

$$
\left(\tilde{\rho}^{\check{B}}(\gamma)-I\right)\left(\check{\Lambda}_{\mathbb{R}, y}\right) \subseteq \mathcal{Q}_{\tau, \mathbb{R}}^{*},
$$

which is what is desired.

3) We will compare the radiance obstruction of the affine structure on $\check{B} \backslash \check{\Delta}^{\prime}$ in $H^{1}\left(\check{B} \backslash \check{\Delta}^{\prime}, \Lambda^{\check{B}_{0}}\right)=H^{1}\left(B \backslash \Delta^{\prime}, \check{\Lambda}^{B_{0}}\right)$ and $c_{1}(\varphi) \in H^{1}\left(B \backslash \Delta^{\prime}, \check{\Lambda}^{B_{0}}\right)$. Write $\check{\Lambda}$ for $\check{\Lambda}^{B_{0}}$. This computation will be sufficient since if $i^{\prime}: B \backslash \Delta^{\prime} \hookrightarrow$ $B_{0}$ and $i: B_{0} \hookrightarrow B$ are the inclusions, then it is easy to see that 
$i_{*}^{\prime}\left(\left.\check{\Lambda}\right|_{B \backslash \Delta^{\prime}}\right)=\check{\Lambda}$ on $B_{0}$, and then by the Leray spectral sequences for $i$ and $i^{\prime}$ we get a series of inclusions

$$
H^{1}\left(B, i_{*} \check{\Lambda}^{B_{0}}\right) \hookrightarrow H^{1}\left(B_{0}, \check{\Lambda}^{B_{0}}\right) \hookrightarrow H^{1}\left(B \backslash \Delta^{\prime}, \check{\Lambda}^{B_{0}}\right) .
$$

Thus if $c_{1}(\varphi)$ and the radiance obstruction coincide in $H^{1}\left(B \backslash \Delta^{\prime}, \check{\Lambda}^{B_{0}}\right)$, then they in fact coincide in $H^{1}\left(B, i_{*} \check{\Lambda}^{B_{0}}\right)$.

To calculate the radiance obstruction and $c_{1}(\varphi)$ on $B \backslash \Delta^{\prime}$, we can use Čech cohomology with respect to the open covering of $B \backslash \Delta^{\prime}$ given by

$$
\left\{\operatorname{Int}(\check{v})=W_{v} \mid v \text { a vertex in } \mathscr{P}\right\} \cup\left\{\operatorname{Int}(\sigma)=W_{\check{\sigma}} \mid \sigma \in \mathscr{P}_{\max }\right\} .
$$

We can choose the zero function as a representative for $\varphi$ on each $\operatorname{Int}(\sigma)$, as $\varphi$ is linear there. On each $W_{v}$, we choose a representative $\varphi_{v}$ for $v$ with $\varphi_{v}(v)=0$; we can then view $\varphi_{v}$ as a piecewise linear function on the fan $\Sigma_{v}$ in $\Lambda_{\mathbb{R}, v}$. As such, $\varphi_{v}$ is given by an element $n_{e} \in \check{\Lambda}_{\mathbb{R}, v}$ for each $e \in \coprod_{v \in \sigma} \operatorname{Hom}(v, \sigma)$ corresponding to maximal cones of $\Sigma_{v}$. The Čech 1-cocycle representing $c_{1}(\varphi)$ then takes the value $n_{e}$ on the connected component of $W_{v} \cap \operatorname{Int}(\sigma)$ corresponding to $e \in \operatorname{Hom}(v, \sigma)$.

On the other hand, to compute the radiance obstruction of $\check{B} \backslash$ $\Delta^{\prime}$, we can use the Cech realisation given in Remark 1.10. There is a canonical coordinate chart $\psi_{\check{\sigma}}: W_{\check{\sigma}} \rightarrow \check{\Lambda}_{\mathbb{R}, \check{\sigma}}^{B_{0}}$ taking $\check{\sigma}$ to zero, the graph of which gives a section of $\mathcal{T}_{W_{\tilde{\sigma}}}$. On the other hand, the choice of function $\varphi_{v}$ determines the polyhedron $\widetilde{v} \subseteq \check{\Lambda}_{\mathbb{R}, v}^{B_{0}}$, with vertices $-n_{e}$ for $e \in \coprod_{v \in \sigma} \operatorname{Hom}(v, \sigma)$. This gives a chart $\psi_{\check{v}}: \operatorname{Int}(\check{v}) \rightarrow \check{\Lambda}_{\mathbb{R}, v}^{B_{0}}$ identifying $\operatorname{Int}(\check{v})$ with $\operatorname{Int}(\widetilde{v})$. Again the graph of this chart gives a section of the tangent bundle $\mathcal{T}_{W_{\tilde{v}}}$. It is then clear, using parallel translation along $e$ to identify $\check{\Lambda}_{\mathbb{R}, v}^{B_{0}}$ and $\check{\Lambda}_{\mathbb{R}, \check{\sigma}}^{B_{0}}$, that the difference of these two sections is $n_{e}$ on the connected component of $W_{v} \cap W_{\check{\sigma}}$ corresponding to $e$. Thus $c_{1}(\varphi)$ and the radiance obstruction coincide.

4) Follows easily from the definitions.

q.e.d.

Finally, we wish to define $\check{\varphi}$, the Legendre transform of $\varphi$. For each $\sigma \in \mathscr{P}$, choose $\tilde{\sigma} \subseteq \Lambda_{\sigma, \mathbb{R}}$ as in Definition 1.38, well-defined up to translation. Let $\check{\varphi}_{\check{\sigma}}$ be the function defined on the normal fan $\check{\Sigma}_{\sigma}$ given by

$$
\check{\varphi}_{\check{\sigma}}(y)=-\inf \{\langle y, x\rangle \mid x \in \tilde{\sigma}\}
$$

for $y \in \Lambda_{\sigma, \mathbb{R}}^{*}$. This is a piecewise linear function on the fan $\check{\Sigma}_{\sigma}=\Sigma_{\check{\sigma}}$, and it is a standard easy fact that it is strictly convex, (see [38], A.3, with opposite sign conventions) with the Newton polytope of $\check{\varphi}_{\check{\sigma}}$ being $\tilde{\sigma}$. If $\tilde{\sigma}$ is changed by a translation, $\check{\varphi}_{\check{\sigma}}$ is changed by a linear function, so it is well-defined modulo linear functions. 
Now let $S_{\check{\sigma}}: U_{\check{\sigma}} \rightarrow \Lambda_{\sigma, \mathbb{R}}^{*}$ be the affine submersion given by the fact that $\check{\mathscr{P}}$ is toric, with $U_{\check{\sigma}}$ sufficiently small. Then we can denote also by $\check{\varphi}_{\check{\sigma}}$ the composition $\check{\varphi}_{\check{\sigma}} \circ S_{\check{\sigma}}$. It is then easy to see

\section{Proposition 1.51.}

1) The $\left(U_{\check{\sigma}}, \check{\varphi}_{\check{\sigma}}\right)$ determine an integral multi-valued piecewise linear function on $\check{B}$ which is strictly convex. We call this $\check{\varphi}$, and say the discrete Legendre transform of $(B, \mathscr{P}, \varphi)$ is $(\check{B}, \check{\mathscr{P}}, \check{\varphi})$.

2) The discrete Legendre transform of $(\check{B}, \check{\mathscr{P}}, \check{\varphi})$ is $(B, \mathscr{P}, \varphi)$.

Example 1.52. Let us relate this version of the discrete Legendre transform to the more traditional form for polyhedral decompositions of $M_{\mathbb{R}}$. We do this by taking $B=M_{\mathbb{R}} / \Gamma$ for some integral lattice $\Gamma \subseteq M$. Then a polyhedral decomposition $\mathscr{P}$ of $B$ is induced by a periodic polyhedral decomposition of $M_{\mathbb{R}}$ (which we will also call $\left.\mathscr{P}\right)$. A multi-valued strictly convex piecewise linear function $\varphi$ on $B$ can then be viewed, by patching together choices of values for $\varphi$, as a singlevalued strictly convex piecewise linear function $\varphi: M_{\mathbb{R}} \rightarrow \mathbb{R}$ satisfying the periodicity condition

$$
\varphi(x+\gamma)=\varphi(x)+\alpha(\gamma)(x),
$$

for some map $\alpha: \Gamma \rightarrow \operatorname{Aff}\left(M_{\mathbb{R}}, \mathbb{R}\right)$. We then define the dual polyhedral decomposition $\check{\mathscr{P}}$ in $N_{\mathbb{R}}$ as follows. For each $\sigma \in \mathscr{P}_{\max }$, let $n_{\sigma} \in N_{\mathbb{R}}$ be the slope of $\left.\varphi\right|_{\sigma}$. For each $\tau \in \mathscr{P}$, let $\check{\tau}$ be the convex hull in $N_{\mathbb{R}}$ of $\left\{n_{\sigma} \mid \tau \subseteq \sigma\right.$ maximal $\}$. It is easy to see that $\check{\mathscr{P}}=\{\check{\tau} \mid \tau \in \mathscr{P}\}$ forms a polyhedral decomposition of $N_{\mathbb{R}}$. Furthermore, $\Gamma$ embeds in $N_{\mathbb{R}}$ by mapping $\gamma \in \Gamma$ to the linear part of $\alpha(\gamma)$. It is easy to see that $\check{\mathscr{P}}$ is periodic with respect to $\Gamma \subseteq N_{\mathbb{R}}$ and hence descends to a decomposition of $\check{B}=N_{\mathbb{R}} / \Gamma$. If $\varphi$ has integral slopes, then $\Gamma \subseteq N$, and $\check{\mathscr{P}}$ is an integral polyhedral decomposition. Finally, we can define the Legendre transform of $\varphi$ by, for $n \in \check{v} \subseteq N_{\mathbb{R}}$ a maximal cell of $\check{\mathscr{P}}$,

$$
\check{\varphi}(n)=\langle n, v\rangle-\varphi(v) .
$$

This in turn defines a multi-valued function $\check{\varphi}$ on $\check{B}$. It is easy to check that $(\check{B}, \check{\mathscr{P}}, \check{\varphi})$ agrees with the discrete Legendre transform defined earlier of $(B, \mathscr{P}, \varphi)$ up to sign conventions.

This discrete Legendre transform appears to be useful in applied mathematics: see for example [10].

Example 1.53. The discrete Legendre transform is also a generalization of Batyrev duality [6]. Let $\Xi \subseteq M_{\mathbb{R}}$ be a reflexive polytope, with 0 the unique interior lattice point. Then $B=\partial \Xi$ has an affine manifold with singularities structure as in Example 1.18. Let $\Sigma$ be the fan in $M_{\mathbb{R}}$ obtained from $\Xi$ by taking $\Sigma$ to consist of cones over proper faces of $\Xi$. Then in fact any piecewise linear function $\varphi$ on $\Sigma$ gives rise to a multi-valued piecewise linear function $\varphi_{B}$ on $B$ as follows. 
For any proper face $\tau$ of $\Xi$, or equivalently $\tau \in \mathscr{P}$, let $W_{\tau}$ be as in Definition 1.25, so that $\left\{W_{\tau}\right\}$ form an open cover of $B$. For each $\tau$, choose $n_{\tau} \in N_{\mathbb{R}}$ such that $\varphi(m)=\left\langle n_{\tau}, m\right\rangle$ for all $m \in \tau$, and let $\varphi_{\tau}: W_{\tau} \rightarrow \mathbb{R}$ be the restriction of $\varphi-n_{\tau}$ to $W_{\tau}$. It is then easy to see that $\varphi_{B}=\left\{\left(W_{\tau}, \varphi_{\tau}\right) \mid \tau \in \mathscr{P}\right\}$ represents a multi-valued piecewise linear function on $B$, strictly convex if $\varphi$ is. If $\varphi$ is integral, then $n_{\tau}$ can be chosen in $N$, and $\varphi_{B}$ is also integral. Note that $\varphi_{B}$ is not the same thing as the restriction of $\varphi$ to $\partial \Xi$.

If $\varphi$ is taken to represent the anti-canonical class of the toric variety defined by $\Sigma$, i.e., $\varphi(v)=1$ for all vertices $v$ of $\Xi$, then $\varphi_{B}$ is strictly convex. As an exercise, check in this case that the discrete Legendre transform of $\left(B, \mathscr{P}, \varphi_{B}\right)$ is obtained as the boundary of the Batyrev dual $\Xi^{*} \subseteq N_{\mathbb{R}}$, defined by

$$
\Xi^{*}=\left\{x \in N_{\mathbb{R}} \mid\langle x, y\rangle \geq-1 \text { for all } y \in \Xi\right\}
$$

with affine structure as in Example 1.18.

1.5. Positivity and simplicity. We will now introduce several possible restrictions on integral affine manifolds with singularities and polyhedral decompositions. These restrictions will be used in $\S \S 4$ and 5, though we will give some motivation here for introducing them.

Let $B$ be an integral affine $n$-dimensional manifold with singularities and $\mathscr{P}$ a toric polyhedral decomposition. We will, once and for all, choose for each $\omega \in \mathscr{P}$ with $\operatorname{dim} \omega=1$ an integral generator $d_{\omega}$ of $\Lambda_{\omega}$. In addition, for each $\rho \in \mathscr{P}$ with $\operatorname{dim} \rho=n-1$, we choose an integral generator $\breve{d}_{\rho}$ of $\mathcal{Q}_{\rho}^{*}$. This is a rank 1 lattice contained naturally in $\check{\Lambda}_{y}$ for any $y \in \operatorname{Int}(\rho) \backslash \Delta$.

These choices give us a canonical ordering of the vertices of $\omega$ or the maximal cells containing $\rho$. Indeed, for $\operatorname{dim} \omega=1$, the morphisms $e: v \rightarrow \omega$ are in one-to-one correspondence with the maximal cones of the dual fan $\check{\Sigma}_{\omega}$ living in the one-dimensional space $\Lambda_{\omega, \mathbb{R}}^{*}$. Then as $d_{\omega} \in \Lambda_{\omega}$, there is a well-defined $e_{\omega}^{+}: v_{\omega}^{+} \rightarrow \omega$ corresponding to the cone on which $d_{\omega}$ is positive, and an $e_{\omega}^{-}: v_{\omega}^{-} \rightarrow \omega$ corresponding to the cone on which $d_{\omega}$ is negative.

The choice of $\breve{d}_{\rho}$ distinguishes the morphisms from $\rho$ to maximal cells: $g_{\rho}^{+}: \rho \rightarrow \sigma_{\rho}^{+}$and $g_{\rho}^{-}: \rho \rightarrow \sigma_{\rho}^{-}$as follows. The fan $\Sigma_{\rho}$ in $\mathcal{Q}_{\rho, \mathbb{R}}$ consists of two cones corresponding to the two different morphisms to maximal cells. We take $g_{\rho}^{+}$to correspond to the cone on which $\check{d}_{\rho}$ is positive and $g_{\rho}^{-}$to that cone on which $\check{d}_{\rho}$ is negative.

Now in general, if $\tau \in \mathscr{P}, f_{i}: v_{i} \rightarrow \tau, e_{i}: \tau \rightarrow \sigma_{i}$ for $i=1,2, v_{i}$ vertices of $\mathscr{P}$ and $\sigma_{i} \in \mathscr{P}_{\max }$, we obtain a loop $\gamma_{f_{1} f_{2}}^{e_{1} e_{2}}$ in $B_{0}$ based at $v_{1}$, by traversing in order the edges $e_{1} \circ f_{1}: v_{1} \rightarrow \sigma_{1}, e_{1} \circ f_{2}: v_{2} \rightarrow \sigma_{1}$ (backwards), $e_{2} \circ f_{2}: v_{2} \rightarrow \sigma_{2}, e_{2} \circ f_{1}: v_{1} \rightarrow \sigma_{2}$ (backwards) of $\operatorname{Bar}(\mathscr{P})$ : 


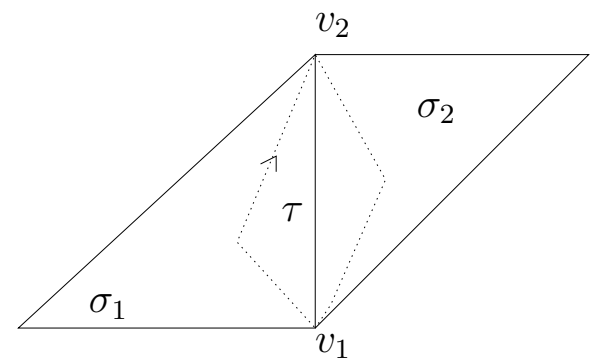

This induces a monodromy transformation

$$
T_{f_{1} f_{2}}^{e_{1} e_{2}}: \Lambda_{v_{1}} \longrightarrow \Lambda_{v_{1}}
$$

via parallel transport around $\gamma_{f_{1} f_{2}}^{e_{1} e_{2}}$. It is often convenient to parallel transport to a point $y \in \operatorname{Int}(\tau) \backslash \Delta$ near $v_{1}$, to get $T_{f_{1} f_{2}}^{e_{1} e_{2}}: \Lambda_{y} \rightarrow \Lambda_{y}$. By Propositions 1.29 and $1.32,\left.T_{f_{1} f_{2}}^{e_{1} e_{2}}\right|_{\Lambda_{\tau}}$ is the identity, and $\left(T_{f_{1} f_{2}}^{e_{1} e_{2}}-I\right)\left(\Lambda_{y}\right) \subseteq$ $\Lambda_{\tau}$.

There are two special cases where this gives useful information. If $\tau=\omega \in \mathscr{P}$ is dimension one, we have $e_{\omega}^{ \pm}: v_{\omega}^{ \pm} \rightarrow \omega$. So given $e_{i}: \omega \rightarrow \sigma_{i}$, $i=1,2$, we get

$$
T_{\omega}^{e_{1} e_{2}}:=T_{e_{\omega}^{+} e_{\omega}^{-}}^{e_{1} e_{2}} .
$$

It follows that there exists an $n_{\omega}^{e_{1} e_{2}} \in \Lambda_{\omega}^{\perp}=\mathcal{Q}_{\omega}^{*} \subseteq \check{\Lambda}_{y}$ such that $T_{\omega}^{e_{1} e_{2}}$ : $\Lambda_{y} \rightarrow \Lambda_{y}$ is given by

$$
T_{\omega}^{e_{1} e_{2}}(m)=m+\left\langle n_{\omega}^{e_{1} e_{2}}, m\right\rangle d_{\omega} .
$$

On the other hand, if $\tau=\rho \in \mathscr{P}$ is dimension $n-1, f_{i}: v_{i} \rightarrow \rho$, we get

$$
T_{f_{1} f_{2}}^{\rho}:=T_{f_{1} f_{2}}^{g_{\rho}^{+} g_{\rho}^{-}} .
$$

Then there exists an $m_{f_{1} f_{2}}^{\rho} \in \Lambda_{\rho} \subseteq \Lambda_{y}$ such that

$$
T_{f_{1} f_{2}}^{\rho}(m)=m+\left\langle\check{d}_{\rho}, m\right\rangle m_{f_{1} f_{2}}^{\rho} .
$$

Combining these two cases, if we are given a map $e: \omega \rightarrow \rho$ with $\operatorname{dim} \omega=1$ and $\operatorname{dim} \rho=n-1$, we can define

$$
T_{e}:=T_{\omega}^{g_{\rho}^{+} \circ e, g_{\rho}^{-} \circ e}=T_{e \circ e_{\omega}^{+}, e \circ e_{\omega}^{-}}^{\rho}
$$

and then we have

$$
n_{e}:=n_{\omega}^{g_{\rho}^{+} \circ e, g_{\rho}^{-} \circ e}, \quad m_{e}:=m_{e \circ e_{\omega}^{+}, e \circ e_{\omega}^{-}}^{\rho} .
$$

Note $n_{e}$ is proportional to $\check{d}_{\rho}$, and $m_{e}$ is proportional to $d_{\omega}$, and the constant of proportionality is the same.

It is also not hard to see that all the monodromy data can be encoded in these numbers. In particular, the $n_{e}\left(\right.$ or $m_{e}$ ) for all $e: \omega \rightarrow \rho$ determine the minimal discriminant locus $\Delta$, if $B$ is obtained from Construction 1.26. Indeed, for $n>2$, if $\tau$ is an $n-2$ dimensional simplex in $\operatorname{Bar}(\mathscr{P})$ contained in $\Delta^{\prime}$ and containing the edge $e: \omega \rightarrow \rho$, then a 
loop about $\tau$ in $B_{0}$ is homotopic (up to orientation) to $\gamma_{e \circ e_{\omega}^{+}, e \circ e_{\omega}^{-}}^{g_{\rho}^{+}, g_{-}^{-}}$. Thus if $n_{e} \neq 0$, all $n-2$ dimensional simplices of $\Delta^{\prime}$ containing $e$ must be included in $\Delta$; if $n_{e}=0$, they are not included in $\Delta$ (see Proposition 1.27). Of course, the same is true for $m_{e}$. For $n=2$ analogous statements hold but now $\omega=\rho$ is an edge.

Definition 1.54. Let $B$ be an integral affine manifold with singularities and a toric polyhedral decomposition $\mathscr{P}$. We say $(B, \mathscr{P})$ is positive if for all $e: \omega \rightarrow \rho$ with $\operatorname{dim} \omega=1, \operatorname{dim} \rho=n-1, m_{e}$ is a non-negative multiple of $d_{\omega}$, or equivalently $n_{e}$ is a non-negative multiple of $\check{d}_{\rho}$.

This may seem mysterious at first, though the importance of positivity will become clearer in $\S 4$. Note that it is independent of the choices of $d_{\omega}$ or $\check{d}_{\rho}$ : indeed, changing the sign of one or the other also changes the orientation of the loop.

This condition can be explained very roughly in dimension two as follows. Given a 2-torus fibration $f: X \rightarrow B$ from an oriented fourmanifold to a two-dimensional base, with degenerate fibres which are pinched tori, there are actually two sorts of such degenerate fibres, distinguished by the sign of the intersection number of the two sheets of the singular fibre at the pinch point. Of course, if $f: X \rightarrow B$ is an elliptic fibration (or special Lagrangian fibration), only the positive case occurs. This places a restriction on the monodromy of the fibration, and it is precisely this restriction that the notion of positivity captures.

Proposition 1.55. If $\varphi$ is a strictly convex multi-valued piecewise linear function on $(B, \mathscr{P}),(\check{B}, \check{\mathscr{P}}, \check{\varphi})$ the discrete Legendre transform of $(B, \mathscr{P}, \varphi)$, then $(B, \mathscr{P})$ is positive if and only if $(\check{B}, \check{\mathscr{P}})$ is positive.

Proof. Under the identification of Proposition 1.50, (1), of $\Lambda^{B_{0}}$ with $\check{\Lambda}^{\check{B}_{0}}$, we can identify, for $y \in \operatorname{Int}(\omega) \backslash \Delta, \Lambda_{\omega}^{B_{0}}$ with $\left(\mathcal{Q}_{\breve{\omega}}^{\check{B}}\right)^{*} \subseteq \check{\Lambda}_{y}^{\check{B}_{0}}$, and thus we can equate a choice of $d_{\omega}$ with a choice of $\breve{d}_{\breve{\omega}}$. Similarly, we can identify $\left(\mathcal{Q}_{\rho}^{B}\right)^{*}$ with $\Lambda_{\check{\rho}}^{\check{B}_{0}}$ for $\check{y} \in \operatorname{Int}(\check{\rho}) \backslash \check{\Delta}$. Thus we can equate a choice of $\check{d}_{\rho}$ with a choice of $d_{\check{\rho}}$.

Now suppose we are given $e: \omega \rightarrow \rho$, and hence $\check{e}: \check{\rho} \rightarrow \check{\omega}$. We are then given $e_{\omega}^{ \pm}: v_{\omega}^{ \pm} \rightarrow \omega$ and $g_{\rho}^{ \pm}: \rho \rightarrow \sigma_{\rho}^{ \pm}$. It is easy to check that $\check{g}_{\rho}^{ \pm}: \check{\sigma}_{\rho}^{ \pm} \rightarrow \check{\rho}$ coincides with $e_{\check{\rho}}^{ \pm}: v_{\check{\rho}}^{ \pm} \rightarrow \check{\rho}$, preserving the sign, and similarly, $\check{e}_{\omega}^{ \pm}: \check{\omega} \rightarrow \check{v}_{\omega}^{ \pm}$coincides with $g_{\check{\omega}}^{ \pm}: \check{\omega} \rightarrow \sigma_{\check{\omega}}^{ \pm}$. Thus in $B$, the monodromy transformation $T_{e}$ is given by transport along the loop $\gamma$ which goes from $v_{\omega}^{+}$to the barycenter of $\sigma_{\rho}^{+}$to $v_{\omega}^{-}$to the barycenter of $\sigma_{\rho}^{-}$to $v_{\omega}^{+}$, while in $\check{B}$, the monodromy transformation $T_{\check{e}}$ is given by transport along $\check{\gamma}$ which goes from $\check{\sigma}_{\rho}^{+}$(the barycenter of $\sigma_{\rho}^{+}$) to the barycenter of $\check{v}_{\omega}^{+}$(i.e., $v_{\omega}^{+}$) to $\check{\sigma}_{\rho}^{-}$to the barycenter of $\check{v}_{\omega}^{-}$and then to $\check{\sigma}_{\rho}^{+}$. But this is precisely the opposite direction of $\gamma$. It then follows from Proposition 1.50, (1), that $T_{\check{e}}=T_{e}^{t}$. Thus if $T_{e}(m)=m+\left\langle n_{e}, m\right\rangle d_{\omega}$ then 
$T_{\check{e}}(n)=n+\left\langle n, d_{\omega}\right\rangle n_{e}$. Thus $m_{\check{e}}=n_{e}$, and one is a positive multiple of $\check{d}_{\rho}$ if and only if the other is.

q.e.d.

Remark 1.56. Positivity can also be interpreted as follows. For a given $\omega \in \mathscr{P}$ with $\operatorname{dim} \omega=1$, the data $n_{\omega}^{e_{1} e_{2}}, e_{i}: \omega \rightarrow \sigma_{i} \in \mathscr{P}_{\max }$ determine a piecewise linear function $\psi_{\omega}$ on the fan $\Sigma_{\omega}$, well-defined up to a linear function, as follows. For any $e: \omega \rightarrow \tau \in \mathscr{P}$, there is a cone in $\Sigma_{\omega}$ corresponding to $e$, which we write as $K_{e}$. Now fix some $e: \omega \rightarrow \sigma \in \mathscr{P}_{\max }$, and take $\left.\psi_{\omega}\right|_{K_{e}}=0$. On any other maximal cone $K_{e^{\prime}} \in \Sigma_{\omega}$, take $\left.\psi_{\omega}\right|_{K_{e^{\prime}}}=-n_{\omega}^{e e^{\prime}}$. We just need to check that this is continuous. If two maximal cones $K_{e_{1}}, K_{e_{2}}$ of $\Sigma_{\omega}$ intersect in a cone $K_{f}$ of $\Sigma_{\omega}$, giving a diagram

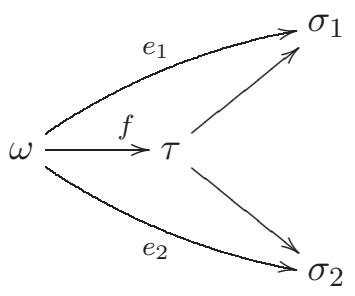

then we need to check $n_{\omega}^{e e_{1}}=n_{\omega}^{e e_{2}}$ on $K_{f}$. But it is easy to see that

$$
T_{\omega}^{e_{1} e_{2}} \circ T_{\omega}^{e e_{1}}=T_{\omega}^{e e_{2}}
$$

and thus

$$
n_{\omega}^{e_{1} e_{2}}=n_{\omega}^{e e_{2}}-n_{\omega}^{e e_{1}} .
$$

But $n_{\omega}^{e_{1} e_{2}} \in \Lambda_{\tau}^{\perp}$ by Proposition 1.29 , so is zero on $K_{f}$. This gives continuity.

Note that changing the initial choice of $e$ just changes $\psi_{\omega}$ by a linear function.

The condition of positivity is now easily seen to be equivalent to $\psi_{\omega}$ being convex for all $\omega$ (though not necessarily strictly convex).

Similarly the data $m_{f_{1} f_{2}}^{\rho}$ determine a piecewise linear function $\check{\psi}_{\rho}$ on the normal fan $\check{\Sigma}_{\rho}$; again, positivity is seen to be equivalent to $\check{\psi}_{\rho}$ convex for all $\rho$.

Example 1.57. One can check that the construction of Example 1.18 only produces positive $(B, \mathscr{P})$. To do this, given $\Xi \subseteq M_{\mathbb{R}}$, $\omega \subseteq \rho$ faces of dimension one and of codimension one respectively, and a choice of $d_{\omega}$ and $\check{d}_{\rho}$, we obtain the endpoints $v_{\omega}^{+}$and $v_{\omega}^{-}$of $\omega$ and the maximal faces $\sigma_{\rho}^{+}$and $\sigma_{\rho}^{-}$containing $\rho$. There exists $n^{+}, n^{-} \in N$ such that $\left\langle n^{+}, \sigma_{\rho}^{+}\right\rangle=\left\langle n^{-}, \sigma_{\rho}^{-}\right\rangle=1$, by reflexivity of $\Xi$. On the other hand, $\Lambda_{v_{\omega}^{+}}$can be identified with $M / v_{\omega}^{+}$, and with $e: \omega \rightarrow \rho$ the unique morphism,

$$
T_{e}(m)=m+\left\langle m, n_{+}-n_{-}\right\rangle\left(v_{\omega}^{-}-v_{\omega}^{+}\right),
$$


as can be computed as in [42], Theorem 8.2, or [23], Lemma 2.4. See also [18], $\S 2$. But since $\Xi$ is convex, $n_{+}-n_{-}$is positive on $\sigma_{\rho}^{+}$. Also, $v_{\omega}^{-}-v_{\omega}^{+}$is a positive multiple of $d_{\omega}$. Hence $B$ is positive.

We will define a certain class of $(B, \mathscr{P})$ which we will call simple. At this point in time, this definition is a bit difficult to motivate. However, it should be viewed as analogous to the notion of simplicity defined in $[\mathbf{1 5}]$ and used extensively in [16] and [17]. Given a torus fibration $f: X \rightarrow B$, with discriminant locus $\Delta, B_{0}=B \backslash \Delta, X_{0}=f^{-1}\left(B_{0}\right)$, $f_{0}=\left.f\right|_{X_{0}}$ and $i: B_{0} \hookrightarrow B$ the inclusion, we said $f$ was $G$-simple for a group $G$ if $i_{*} R^{p} f_{0 *} G=R^{p} f_{*} G$ for all $p$. This essentially says that the cohomology of any singular fibre is isomorphic to the monodromy invariant part of the cohomology of a nearby non-singular fibre. It was only with this assumption that the Strominger-Yau-Zaslow conjecture implies the usual connection between $h^{1,1}$ and $h^{1,2}$ of mirror pairs of Calabi-Yau threefolds. If the fibrations are not simple, the connection between these numbers is more tenuous, and in particular we may not expect a clear isomorphism between complexified Kähler moduli and complex moduli of the mirror.

One would like some analogue of simplicity for affine manifolds with singularities. We cannot use the definition directly, because we don't have torus fibrations. Instead we will use a definition which places restrictions on the monodromy of the local system $\Lambda$ on standard open sets $W_{\tau} \cap B_{0}$. In dimensions two and three, this will essentially coincide with the restrictions on monodromy implied by the old definition of simplicity. However, our new notion of simplicity also depends on $\mathscr{P}$ : the polyhedral decomposition determines the "scale" of detail we see in the discriminant locus. The definition of simplicity only depends on the monodromy transformations $T_{e}$ for various $e$. For example, if $\operatorname{dim} B=2$ and there are several points in $\Delta$ on a single edge, simplicity places a restriction on the monodromy of a loop enclosing all these points, and not on loops around each point on the edge. So even in the two-dimensional case, simplicity in the old sense only implies simplicity in the new sense if $\mathscr{P}$ is sufficiently fine. Again, it is useful to assume the discriminant locus has already been straightened to avoid this problem. In any event, with these caveats, our definition clearly gives the right thing in dimensions two and three, and we shall see more clearly the naturality of this definition in $\S 5$, as well as in $[\mathbf{1 8}]$ and $[\mathbf{2 1}]$.

Before giving our definition of simplicity, we define some auxiliary polytopes associated with $(B, \mathscr{P})$ which encode key data about the monodromy.

Definition 1.58. For a given $\omega \in \mathscr{P}$ with $\operatorname{dim} \omega=1$, fix $e: \omega \rightarrow \sigma \in$ $\mathscr{P}_{\text {max }}$, and let $\breve{\Delta}(\omega)$ be the convex hull in $\Lambda_{\omega, \mathbb{R}}^{\perp} \subseteq \check{\Lambda}_{\mathbb{R}, y}$ (for $y \in \operatorname{Int}(\omega) \backslash \Delta$ 
near $v_{\omega}^{+}$) of the set

$$
\left\{n_{\omega}^{e e^{\prime}} \mid e^{\prime}: \omega \rightarrow \sigma^{\prime} \in \mathscr{P}_{\max }\right\} .
$$

Similarly, for a given $\rho$, fix $f: v \rightarrow \rho$ and let $\Delta(\rho)$ be the convex hull in $\Lambda_{\rho, \mathbb{R}} \subseteq \Lambda_{\mathbb{R}, y}($ for $y \in \operatorname{Int}(\rho) \backslash \Delta$ near $v)$ of $\left\{m_{f f^{\prime}}^{\rho} \mid f^{\prime}: v^{\prime} \rightarrow \rho\right\}$.

These polytopes are well-defined up to translation, independent of the choice of $\sigma$ or $v$.

If $\tau \in \mathscr{P}$ with $1 \leq \operatorname{dim} \tau \leq n-1$, we can also define auxiliary polytopes related to $\tau$. Given $e: \omega \rightarrow \tau$ with $\operatorname{dim} \omega=1$, choose some $f: \tau \rightarrow \sigma \in \mathscr{P}_{\max }$, and set $\check{\Delta}_{e}(\tau)$ to be the convex hull of

$$
\left\{n_{\omega}^{f \circ e, f^{\prime} \circ e} \mid f^{\prime}: \tau \rightarrow \sigma^{\prime} \in \mathscr{P}_{\max }\right\}
$$

in $\Lambda_{\tau, \mathbb{R}}^{\perp}=\mathcal{Q}_{\tau, \mathbb{R}}^{*} \subseteq \check{\Lambda}_{\mathbb{R}, y}$, for $y \in \operatorname{Int}(\tau) \backslash \Delta$ near $v_{\omega}^{+}$. Thus $\check{\Delta}_{e}(\tau)$ is just a face of $\check{\Delta}(\omega)$.

Similarly, given $e: \tau \rightarrow \rho \operatorname{dim} \rho=n-1$, choose some $f: v \rightarrow \tau$ and set $\Delta_{e}(\tau)$ to be the convex hull of $\left\{m_{e \circ f, e \circ f^{\prime}}^{\rho} \mid f^{\prime}: v^{\prime} \rightarrow \tau\right\}$. Again, $\Delta_{e}(\tau)$ is a face of $\Delta(\rho)$.

\section{Remark 1.59.}

1) These polytopes are only of interest in the positive case, in which case $\check{\Delta}(\omega)$ and $\Delta(\rho)$ can be viewed as the Newton polytopes determined by $\psi_{\omega}$ and $\check{\psi}_{\rho}$ (Remark 1.56$)$ respectively. In this case, $\psi_{\omega}$ can be recovered, (up to a linear function) as

$$
\psi_{\omega}(y)=-\inf \{\langle y, x\rangle \mid x \in \check{\Delta}(\omega)\}
$$

and similarly

$$
\varphi_{\rho}(x)=-\inf \{\langle y, x\rangle \mid y \in \Delta(\rho)\} .
$$

Furthermore, by construction of $\psi_{\omega}$, if $e_{i}: \omega \rightarrow \sigma_{i} \in \mathscr{P}_{\max }, i=1,2$ define cones $K_{e_{i}} \in \Sigma_{\omega}$, and $\psi_{\omega}$ plus any linear function is given by $-p_{e_{i}}$ on $K_{e_{i}}$, then $n_{\omega}^{e_{1} e_{2}}=p_{e_{2}}-p_{e_{1}}$. Thus all the $n_{\omega}^{e_{1} e_{2}}$ 's can be recovered from knowing $\breve{\Delta}(\omega)$ in the positive case, and similarly the $m_{e_{1} e_{2}}^{\rho}$ 's can be recovered from $\Delta(\rho)$ for $e_{i}: v_{i} \rightarrow \rho$.

The same comments hold for the $\check{\Delta}_{e}(\tau)$ and $\Delta_{e}(\tau)$ : given $e: \omega \rightarrow \tau$ with $\operatorname{dim} \omega=1$, the $n_{\omega}^{f_{1} \circ e, f_{2} \circ e}$ can be recovered from $\check{\Delta}_{e}(\tau)$ for $f_{i}: \tau \rightarrow$ $\sigma_{i} \in \mathscr{P}_{\max }$; similarly given $f: \tau \rightarrow \rho$ with $\operatorname{codim} \rho=1$, the $m_{f_{\circ} e_{1}, f \circ e_{2}}$ can be recovered from $\Delta_{e}(\tau)$ for $e_{i}: v_{i} \rightarrow \tau$.

2) From these remarks it also follows readily that $\Sigma_{\omega}$ is a refinement of the normal fan of $\check{\Delta}(\omega)$. The normal vector $\check{d}_{\rho}$ of a codimension-one cell $\tilde{\rho}$ containing $\tilde{\omega}$ is parallel to an edge of $\check{\Delta}(\omega)$ if $n_{e} \neq 0$ for $e: \omega \rightarrow \rho$ the corresponding morphism. This is the case iff the corresponding edge ( $n=2$ : vertex) of the barycentric decomposition is contained in $\Delta$.

Similarly, $\check{\Sigma}_{\rho}$ is a refinement of the normal fan of $\Delta(\rho)$. Hence $\Delta(\rho)$ is a polytope with faces parallel to faces of $\tilde{\rho} \subset \Lambda_{\rho, \mathbb{R}}$ itself. Again, an 
edge $\tilde{\omega} \subset \tilde{\rho}$ occurs in this list if the corresponding edge of the barycentric subdivision is part of $\Delta$.

Note that if all $n_{e}$ (or, equivalently, all $m_{e}$ ) are primitive it follows that all these polytopes are determined uniquely just by $\mathscr{P}$ and the combinatorics of the discriminant locus relative to $\mathscr{P}$.

Definition 1.60. Let $B$ be an integral affine manifold with singularities and $\mathscr{P}$ a toric polyhedral decomposition of $B$. Suppose $(B, \mathscr{P})$ is positive. Then we say $(B, \mathscr{P})$ is simple if for every $\tau \in \mathscr{P}$ with $1 \leq \operatorname{dim} \tau \leq n-1$, the following condition holds:

Let

$$
\mathscr{P}_{1}(\tau)=\{e: \omega \rightarrow \tau \mid \operatorname{dim} \omega=1\}
$$

and

$$
\mathscr{P}_{n-1}(\tau)=\{f: \tau \rightarrow \rho \mid \operatorname{dim} \rho=n-1\} .
$$

Then there exist disjoint subsets

$$
\Omega_{1}, \ldots, \Omega_{p} \subseteq \mathscr{P}_{1}(\tau)
$$

and

$$
R_{1}, \ldots, R_{p} \subseteq \mathscr{P}_{n-1}(\tau)
$$

for some $p \geq 0$ such that

1) For $e \in \mathscr{P}_{1}(\tau)$ and $f \in \mathscr{P}_{n-1}(\tau), n_{f \circ e}=0$ unless $e \in \Omega_{i}, f \in R_{i}$ for some $i$.

2) For each $1 \leq i \leq p, \check{\Delta}_{e}(\tau)$ is independent (up to translation) of $e \in \Omega_{i}$, and we denote $\breve{\Delta}_{e}(\tau)$ by $\check{\Delta}_{i}$ for $e \in \Omega_{i}$; similarly, $\Delta_{f}(\tau)$ is independent (up to translation) of $f \in R_{i}$, and we denote $\Delta_{f}(\tau)$ by $\Delta_{i}$.

3) Let $e_{1}, \ldots, e_{p}$ be the standard basis of $\mathbb{Z}^{p}$, and let $\check{\Delta}(\tau)$ be the convex hull of

$$
\bigcup_{i=1}^{p} \check{\Delta}_{i} \times\left\{e_{i}\right\}
$$

in $\left(\mathcal{Q}_{\tau}^{*} \oplus \mathbb{Z}^{p}\right) \otimes \mathbb{R}$. Then $\check{\Delta}(\tau)$ is an elementary simplex of some dimension (i.e., a simplex whose only integral points are its vertices). Similarly, if $\Delta(\tau)$ is the convex hull of

$$
\bigcup_{i=1}^{p} \Delta_{i} \times\left\{e_{i}\right\}
$$

in $\left(\Lambda_{\tau} \oplus \mathbb{Z}^{p}\right) \otimes \mathbb{R}$, then $\Delta(\tau)$ is an elementary simplex.

These last two conditions are vacuous if $p=0$.

In case $\tau$ is one-dimensional the polytope $\check{\Delta}(\tau)$ agrees with the polytope defined in Definition 1.58, and similarly in the one-codimensional case with $\Delta(\tau)$. Thus the notation is consistent with previous usage. 
Remark 1.61. We give a number of elementary observations related to this definition, which will hopefully build some feeling for a rather complex situation. We always suppose that $(B, \mathscr{P})$ is positive and simple.

1) For any $\tau \in \mathscr{P}$ with $0<\operatorname{dim} \tau<\operatorname{dim} B, e_{i}: \tau \rightarrow \sigma_{i} \in \mathscr{P}_{\max }$, $f: \omega \rightarrow \tau$ with $\operatorname{dim} \omega=1, f \in \Omega_{i}$, then $n_{\omega}^{e_{1} \circ f, e_{2} \circ f}$ is independent of $f \in \Omega_{i}$. Indeed, by Remark 1.59, $n_{\omega}^{e_{1} \circ f, e_{2} \circ f}$ is determined by $\check{\Delta}_{f}(\tau)=\check{\Delta}_{i}$, independently of $f \in \Omega_{i}$. Similarly for $e: \tau \rightarrow \rho$ with $\operatorname{codim} \rho=1, e \in R_{i}, f_{i}: v_{i} \rightarrow \tau, m_{e \circ f_{1}, e \circ f_{2}}^{\rho}$ is independent of $e$ in $R_{i}$.

2) If $f: \omega \rightarrow \tau$ is not in $\bigcup_{i=1}^{p} \Omega_{i}$ then $n_{\omega}^{e \circ f, e^{\prime} \circ f}=0$. In fact, by (1) in the definition, $n_{g}=0$ for all $g: \omega \rightarrow \rho$ factoring through $f$ for $\operatorname{dim} \rho=n-1$. In general there exists a sequence $g_{1}, \ldots, g_{m}, g_{i}$ : $\omega \rightarrow \rho_{i}$ factoring through $f, \operatorname{dim} \rho_{i}=n-1$, such that $T_{\omega}^{e o f, e^{\prime} \circ f}=$ $T_{g_{1}}^{ \pm 1} \circ \cdots \circ T_{g_{m}}^{ \pm 1}$, hence $n_{\omega}^{e \circ f, e^{\prime} \circ f}= \pm n_{g_{1}} \pm \cdots \pm n_{g_{m}}=0$.

3) By taking $p$ to be as small as possible, we can assume that $n_{g \circ f} \neq$ $0, m_{g \circ f} \neq 0$ for any $g \in R_{i}, f \in \Omega_{i}$. Indeed, by (1), if $n_{g \circ f}=0$ for some $f \in \Omega_{i}$, in fact $n_{g \circ f}=0$ for all $f \in \Omega_{i}$. But if $n_{g \circ f}=0$, we also have $m_{g \circ f}=0$, so $m_{g \circ f}=0$ for all $g \in R_{i}$. Thus $n_{g \circ f}=$ $m_{g \circ f}=0$ for all $g \in R_{i}, f \in \Omega_{i}$. So we could just as well leave off $R_{i}$ and $\Omega_{i}$, and condition (1) of Definition 1.60 still holds.

Note also we can always assume $\operatorname{dim} \breve{\Delta}_{i}>0$ and $\operatorname{dim} \Delta_{i}>0$, for if one of these is a point, then $n_{g \circ f}=0$ for all $g \in R_{i}, f \in \Omega_{i}$.

4) Let $\Xi \subseteq M_{\mathbb{R}}$ be an elementary simplex, and $\check{\Delta}_{1}, \ldots, \check{\Delta}_{p} \subseteq \Xi$ disjoint faces. Let $T_{\check{\Delta}_{i}} \subseteq M_{\mathbb{R}}$ be the tangent space to $\check{\Delta}_{i}$. It is easy to see that $T_{\check{\Delta}_{1}}+\cdots+T_{\check{\Delta}_{p}}$ forms an internal direct sum in $M_{\mathbb{R}}$ (check this for a standard simplex!) Thus, in the case of Definition 1.60, if $T_{\check{\Delta}_{i}} \subseteq \mathcal{Q}_{\tau, \mathbb{R}}^{*}$ is the tangent space to $\check{\Delta}_{i}$, then since $\check{\Delta}_{1} \times\left\{e_{1}\right\}, \ldots, \check{\Delta}_{p} \times\left\{e_{p}\right\}$ are disjoint faces of $\check{\Delta}(\tau), T_{\check{\Delta}_{1}}+\cdots+T_{\check{\Delta}_{p}}$ is an internal direct sum in $\mathcal{Q}_{\tau, \mathbb{R}}^{*}$. The dual statement holds for the $\Delta_{i}$.

5) If $p$ is taken to be as small as possible as in (2), then $p \leq$ $\min (\operatorname{dim} \tau, \operatorname{codim} \tau)$. Indeed, $\operatorname{dim} \mathcal{Q}_{\tau, \mathbb{R}}^{*}=\operatorname{codim} \tau$, and by the assumption $p$ is as small as possible, $\operatorname{dim} \check{\Delta}_{i} \geq 1$ for all $i$ by (2). Thus by (4), $p \leq \operatorname{codim} \tau$. Dually, $p \leq \operatorname{dim} \tau$.

Example 1.62. Let us consider what simplicity means for $\operatorname{dim} B \leq$ 3. It is vacuous if $\operatorname{dim} B=1$. For $\operatorname{dim} B=2$, let $\tau \in \mathscr{P}$ with $\operatorname{dim} \tau=1$. Then

$$
\mathscr{P}_{1}(\tau)=\mathscr{P}_{n-1}(\tau)=\{i d: \tau \rightarrow \tau\} .
$$

If $n_{i d}=0$, i.e., if $\operatorname{Int}(\tau) \cap \Delta=\emptyset$, then we take $p=0$, and conditions (1)-(3) are vacuous. 
If $n_{i d} \neq 0$, then $n_{i d}=n \cdot \check{d}_{\omega}$ for some $n \in \mathbb{Z}, n>0$ by positivity, and we take $\Omega_{1}=R_{1}=\{i d\}$, and $\breve{\Delta}(\tau)$ and $\Delta(\tau)$ are both line segments of length $n$. (The monodromy operator $T_{i d}$ is $\left(\begin{array}{ll}1 & n \\ 0 & 1\end{array}\right)$ in an appropriate basis). Then the condition that $\check{\Delta}(\tau) \times\left\{e_{1}\right\}$ and $\Delta(\tau) \times\left\{e_{1}\right\}$ are elementary simplices is just stating that $n=1$. Thus, if the discriminant locus of $B$ is straightened, simplicity is equivalent to monodromy about each point in $\Delta$ being $\left(\begin{array}{ll}1 & 1 \\ 0 & 1\end{array}\right)$.

In dimension 3 , consider first $\operatorname{dim} \tau=1$, so $\mathscr{P}_{1}(\tau)=\{i d\}$. Then either $n_{e}=0$ for all $e \in \mathscr{P}_{2}(\tau)$, in which case we take $p=0$, and then $\tau \cap \Delta=\emptyset$, or else we have to take $\Omega_{1}=\mathscr{P}_{1}(\tau), R_{1}=\left\{e \in \mathscr{P}_{2}(\tau) \mid n_{e} \neq\right.$ $0\}$. In the latter case, condition (1) is satisfied, and (2) and (3) together imply $\Delta_{e}(\tau)$ is a line segment of length 1 for each $e \in R_{1}$, while $\breve{\Delta}(\tau)$ is a standard simplex of dimension 1 or 2 . If $\operatorname{dim} \check{\Delta}(\tau)=1$, it is then easy to see that near $\tau, \Delta$ is just a line segment. By the definition of polyhedral decomposition, $\Delta$ must be contained in a union of 2-cells of $\mathscr{P}$. However, each 2-cell containing a one-dimensional part of this piece of the discriminant locus must lie in the unique (local) affine plane containing $\Delta$ which is invariant under holonomy, and this plane contains $\tau$, as depicted below. If $\operatorname{dim} \check{\Delta}(\tau)=2, \Delta$ is just a trivalent graph, as depicted.

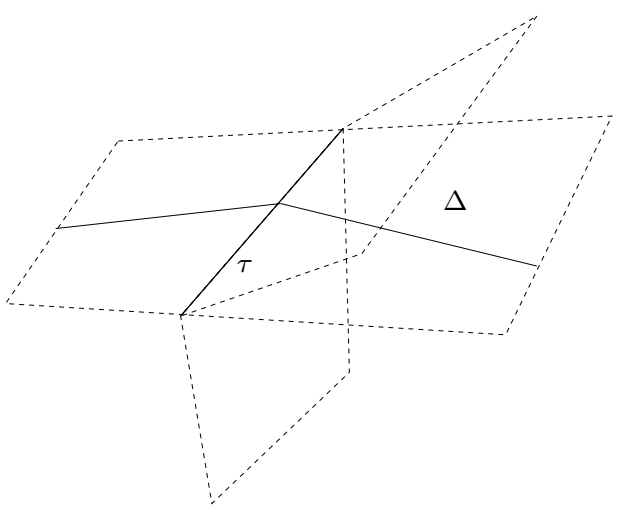

Bivalent

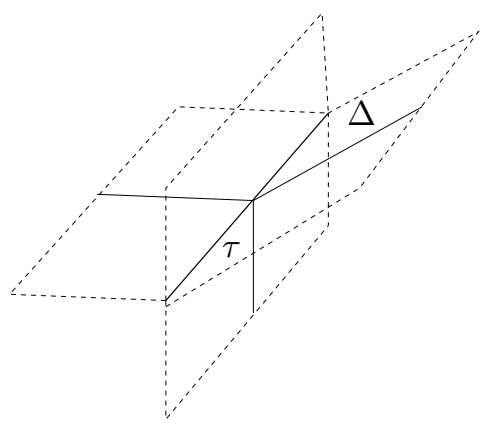

Trivalent

Furthermore, in a suitable basis with $d_{\tau}=(1,0,0)$, the monodromy about $\Delta$ in the first case is

$$
\left(\begin{array}{lll}
1 & 1 & 0 \\
0 & 1 & 0 \\
0 & 0 & 1
\end{array}\right),
$$


whereas in the second case, the monodromy about the three legs is

$$
\left(\begin{array}{lll}
1 & 1 & 0 \\
0 & 1 & 0 \\
0 & 0 & 1
\end{array}\right),\left(\begin{array}{lll}
1 & 0 & 1 \\
0 & 1 & 0 \\
0 & 0 & 1
\end{array}\right) \text {, and }\left(\begin{array}{ccc}
1 & -1 & -1 \\
0 & 1 & 0 \\
0 & 0 & 1
\end{array}\right)
$$

respectively.

For $\operatorname{dim} \tau=2$, the picture is very similar, with two pictures occuring depending on the dimension of $\Delta(\tau)$, namely

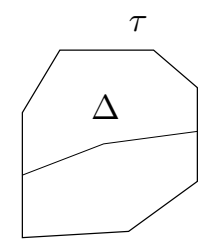

or

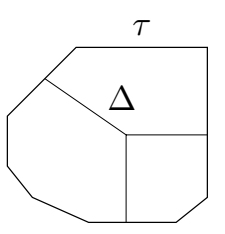

and the monodromy is the transpose of the ones appearing in the $\operatorname{dim} \tau=1$ case. This agrees with the behaviour of semi-stable, simple torus fibrations discussed in [17]. However, in that paper, another type of behaviour occured. It was possible to have a semi-stable, simple torus fibration with a quadrivalent vertex, over which occured a fibre of type $(1,1)$. Notice that we do not have quadrivalent vertices in our situation; it appears that the straightening process would remove such a vertex, replacing it by two trivalent vertices.

The role of the partition really only becomes important in dimension 4 and higher. Rather than give a complete description in the fourdimensional case, we just give an example of the behaviour we expect. We may have, for example, $\tau \in \mathscr{P}$ with $\operatorname{dim} \tau=2, \tau$ a parallelogram, say with vertices $(0,0),(1,0),(a, b)$, and $(a+1, b)$, where $\operatorname{gcd}(a, b)=1$, $b \neq 0$. On the other hand, we may have $\Sigma_{\tau}$ the two-dimensional fan with 1-dimensional cones generated by $(\check{b},-\check{a}),(-\check{b}, \check{a}),(0,1)$ and $(0,-1)$, where again $\operatorname{gcd}(\check{a}, \breve{b})=1$ and $\breve{b} \neq 0$. Then it is possible to arrange monodromy so that the definition of simplicity is satisfied, using the following choice of $\Omega_{i}, R_{i}$. We take $\Omega_{1}$ to be the two parallel edges of $\tau$ joining $(0,0)$ with $(1,0)$, and $(a, b)$ with $(a+1, b)$, while $\Omega_{2}$ consists of the two other edges of $\tau$. Meanwhile, $R_{1}$ consists of the two 3-cells corresponding to the rays of $\Sigma_{\tau}$ generated by $(0, \pm 1)$, while $R_{2}$ consists of the two 3 -cells corresponding to the rays generated by $\pm(\check{b},-\check{a})$. Furthermore, we may then have $\breve{\Delta}_{1}$ being the line segment with endpoints $(0,0)$ and $(1,0) ; \check{\Delta}_{2}$ the line segment with endpoints $(0,0)$ and $(\check{a}, \check{b})$; while $\Delta_{1}$ is the line segment with endpoints $(0,0)$ and $(1,0) ; \Delta_{2}$ is the line segment with endpoints $(0,0)$ and $(a, b)$. If this is the case, then the definition of simplicity is in fact satisfied. Furthermore, in this case, near $\tau$ the discriminant locus consists of two two-dimensional manifolds intersecting at a point. This is in fact the sort of behaviour we would expect for the discriminant locus of an abelian surface fibration over a 
complex surface, so it is quite possible this sort of picture would appear in the study of degenerations of hyperkähler manifolds.

Note in this case that the monodromy about the two branches of the discriminant locus takes the form, in an appropriate basis,

$$
\left(\begin{array}{cccc}
1 & 0 & 1 & 0 \\
0 & 1 & 0 & 0 \\
0 & 0 & 1 & 0 \\
0 & 0 & 0 & 1
\end{array}\right) \text { and }\left(\begin{array}{cccc}
1 & 0 & \check{a} a & \check{b} a \\
0 & 1 & \check{a} b & \check{b} b \\
0 & 0 & 1 & 0 \\
0 & 0 & 0 & 1
\end{array}\right) \text {. }
$$

Example 1.63. The $(B, \mathscr{P})$ constructed in Example $1.23(2)$ are not in general simple. To get simple $(B, \mathscr{P})$ from reflexive polytopes, one must use a more sophisticated construction. This construction has in fact been given by Haase and Zharkov in [23]. In the notation of that paper, simplicity is implied by a sufficiently generic choice of the vectors $\lambda$ and $\nu$, which will correspond to choosing ample polarizations on maximally projective crepant resolutions of the toric varieties $\mathbb{P}_{\Xi}$ and $\mathbb{P}_{\Xi^{*}}$. This is shown in [18], where the more general case of complete intersections in toric varieties is considered. So one could think of simplicity as a sort of ampleness condition.

\section{From polyhedral decompositions to algebraic spaces}

In this section, given an integral affine manifold with singularities $B$ with toric polyhedral decomposition $\mathscr{P}$, we will construct algebraic spaces obtained by gluing toric varieties.

We fix an integral affine manifold with singularities $B$, and a toric polyhedral decomposition $\mathscr{P}$ once and for all in this section. There are two constructions we can make, depending on whether we view $B$ as an intersection complex or a dual intersection complex of the central fibre of a degeneration. We shall first consider the case where we view $B$ as an intersection complex, in which case the maximal cells of $\mathscr{P}$ correspond to irreducible components of a scheme, with a polarisation. This is

conceptually simpler than the dual picture, as we shall see, but should be viewed as somewhat less important for our point of view. We refer to this construction as the cone picture, and will refer to the dual construction, in which vertices of $\mathscr{P}$ correspond to irreducible components, as the fan picture.

Whenever $P$ is a monoid, $A$ a ring, we denote by $A[P]$ the monoid algebra over $A$ defined by

$$
A[P]=\bigoplus_{m \in P} A \cdot z^{m}
$$

with

$$
z^{m} \cdot z^{m^{\prime}}=z^{m+m^{\prime}}
$$




\subsection{The cone picture.}

Definition 2.1. If $\sigma \in \mathscr{P}$, let $\tilde{\sigma} \subseteq \Lambda_{\sigma, \mathbb{R}}$ be as in Definition 1.38, well-defined up to translation. Set

$$
C(\sigma)=\left\{(r p, r) \mid r \in \mathbb{R}_{\geq 0}, p \in \tilde{\sigma}\right\} \subseteq \Lambda_{\sigma, \mathbb{R}} \oplus \mathbb{R}
$$

to be the cone over $\tilde{\sigma}$. Let $\check{P}_{\sigma}$ be the monoid $C(\sigma) \cap\left(\Lambda_{\sigma} \oplus \mathbb{Z}\right)$. The monoid $\check{P}_{\sigma}$ is well-defined up to unique isomorphism: if $\tilde{\sigma}$ is translated, there is a well-defined isomorphism identifying the two choices of $\check{P}_{\sigma}$. The projection $\check{P}_{\sigma} \rightarrow \mathbb{Z}$ defines a $\mathbb{Z}$-grading on the monoid ring $R_{\sigma}:=\mathbb{Z}\left[\check{P}_{\sigma}\right]$. Define

$$
\check{X}_{\sigma}:=\operatorname{Proj} R_{\sigma}
$$

This also defines a line bundle $\mathcal{O}_{\check{X}_{\sigma}}(1)$ on $\check{X}_{\sigma}$.

Note that $R_{\sigma}$ and $\check{X}_{\sigma}$ are well-defined up to isomorphism.

We can define a contravariant functor $\check{F}$ from $\underline{\operatorname{Cat}}(\mathscr{P})$ to the category of graded rings as follows. We take $\check{F}(\tau)=R_{\tau}$. If $\tau \subseteq \sigma$, then $e \in$ $\operatorname{Hom}(\tau, \sigma)$ determines a face $\tilde{\tau}$ of $\tilde{\sigma} \subseteq \Lambda_{\sigma, \mathbb{R}}$, so that the edge of the barycentric subdivision of $\tilde{\sigma}$ joining the barycenters of $\tilde{\sigma}$ and $\tilde{\tau}$ maps to $e$. This face $\tilde{\tau}$ can be identified, by translation, with $\tilde{\tau} \subseteq \Lambda_{\tau, \mathbb{R}}$. This then gives a well-defined inclusion of $\check{P}_{\tau}$ in $\check{P}_{\sigma}$. We then define $\check{F}(e)$ to be the graded ring homomorphism $\mathbb{Z}\left[\check{P}_{\sigma}\right] \rightarrow \mathbb{Z}\left[\check{P}_{\tau}\right]$ defined by

$$
z^{m} \longmapsto \begin{cases}0 & m \notin \check{P}_{\tau} \\ z^{m} & m \in \check{P}_{\tau} .\end{cases}
$$

Example 2.2. Let $B=\mathbb{R}^{2} / \mathbb{Z}^{2}$, with $M=\mathbb{Z}^{2}$, and take as decomposition $\mathscr{P}$ of $B$ the decomposition with only one maximal cell, coming from the unit square in $\mathbb{R}^{2}$. The set $\mathscr{P}$ consists of one square $\sigma$, two edges $\tau_{1}, \tau_{2}$, and one point $v$. We see

$$
R_{\sigma}=\mathbb{Z}[x, y, z, w] /(x y-z w)
$$

and $\check{X}_{\sigma}=\mathbb{P}^{1} \times \mathbb{P}^{1}$, while $R_{\tau_{1}}=\mathbb{Z}[u, v], R_{\tau_{2}}=\mathbb{Z}[s, t]$ and $R_{v}=\mathbb{Z}[q]$. The two different maps $R_{\sigma} \rightarrow R_{\tau_{1}}$ are given by

$$
x \mapsto u, z \mapsto v, y \mapsto 0, w \mapsto 0
$$

and

$$
x \mapsto 0, z \mapsto 0, y \mapsto v, w \mapsto u
$$

respectively, and the two different maps $R_{\sigma} \rightarrow R_{\tau_{2}}$ are given by

$$
x \mapsto s, w \mapsto t, y \mapsto 0, z \mapsto 0
$$

and

$$
x \mapsto 0, w \mapsto 0, y \mapsto t, z \mapsto s
$$

respectively. 
The inverse limit of $\check{F}$ exists in the category of graded rings. (See [33], III.3 and III.4 for the notions of inverse and direct limits of functors.) Calling this limit the graded $\operatorname{ring} R=: \lim \check{F}$, we get a scheme $\check{X}_{0}(B, \mathscr{P})=\operatorname{Proj} R$, along with a line bundle $\widetilde{\mathcal{O}}_{\check{X}_{0}(B, \mathscr{P})}(1)$. If $\mathscr{P}$ is finite, then $R$ is a finitely generated $\mathbb{Z}$-algebra and Proj $R$ is a projective scheme with $\mathcal{O}_{\check{X}_{0}(B, \mathscr{P})}(1)$ ample. The normalization of $\check{X}_{0}(B, \mathscr{P})$ consists of $\coprod_{\sigma \in \mathscr{P} \max } \check{X}_{\sigma}$. However, we would also like a twisted version of this construction, so that we get an entire family of gluings. We will do this over a ring $A$.

Definition 2.3. Let $A$ be a ring. Then gluing data (for the cone picture) for $\mathscr{P}$ over $A$ are data $\check{s}=\left(\check{s}_{e}\right)_{e \in \amalg_{\tau, \sigma \in \mathscr{P}} \operatorname{Hom}(\tau, \sigma)}$, where if $e$ : $\tau \rightarrow \sigma$ then $\check{s}_{e} \in \operatorname{Hom}\left(\check{P}_{\tau}, \mathbb{G}_{m}(A)\right)$, satisfying the conditions

1) $\check{s}_{i d}(p)=1$ for all $p \in \check{P}_{\sigma}, i d: \sigma \rightarrow \sigma$ the identity.

2) If $e_{1}: \sigma_{1} \rightarrow \sigma_{2}$ and $e_{2}: \sigma_{2} \rightarrow \sigma_{3}, e_{3}=e_{2} \circ e_{1}$, then $\left.\check{s}_{e_{1}} \cdot \check{s}_{e_{2}}\right|_{\check{P}_{\sigma_{1}}}=\check{s}_{e_{3}}$ in $\operatorname{Hom}\left(\check{P}_{\sigma_{1}}, \mathbb{G}_{m}(A)\right)$.

Given such gluing data, we can then define a contravariant functor $\check{F}_{A, \check{s}}$ from $\underline{\operatorname{Cat}}(\mathscr{P})$ to the category of graded $A$-algebras via

$$
\check{F}_{A, \check{s}}(\sigma)=R_{\sigma} \otimes_{\mathbb{Z}} A
$$

and if $e: \sigma \rightarrow \tau$ then

$$
\check{F}_{A, \check{s}}(e)\left(z^{p} \otimes 1\right)=\check{F}(e)\left(z^{p}\right) \otimes \check{s}_{e}(p) .
$$

Definition 2.4. Given gluing data $\check{s}$, we let

$$
\check{X}_{0}(B, \mathscr{P}, \check{s})=\operatorname{Proj}\left(\lim _{\longleftarrow} \check{F}_{A, \check{s}}\right) .
$$

If $\mathscr{P}$ is finite, this is a projective scheme over $\operatorname{Spec} A$, with ample line bundle $\mathcal{O}_{\check{X}_{0}(B, \mathscr{P}, \check{s})}(1)$.

Example 2.5. Continuing with Example 2.2, we can take $A=k$ an algebraically closed field. Using arbitrary gluing data, we get four maps

$$
R_{\sigma} \otimes k \longrightarrow R_{v} \otimes k
$$

given by

$$
\begin{aligned}
x \mapsto \lambda_{x} q, & y, z, w \mapsto 0 \\
y & \mapsto \lambda_{y} q, \quad x, z, w \mapsto 0 \\
z & \mapsto \lambda_{z} q, \quad x, y, w \mapsto 0 \\
w & \mapsto \lambda_{w} q, \quad x, y, z \mapsto 0 .
\end{aligned}
$$

The two maps

$$
R_{\tau_{1}} \otimes k \longrightarrow R_{v} \otimes k
$$


are given by

$$
\begin{aligned}
u \mapsto \lambda_{1} q, \quad v \mapsto 0 \\
u \mapsto 0, \quad v \mapsto \lambda_{2} q
\end{aligned}
$$

and the two maps

$$
R_{\tau_{2}} \otimes k \longrightarrow R_{v} \otimes k
$$

are given by

$$
\begin{aligned}
s \mapsto \mu_{1} q, \quad t & \mapsto 0 \\
s & \mapsto 0, \quad t \mapsto \mu_{2} q .
\end{aligned}
$$

The cocycle condition of Definition 2.3 then dictates that the maps $R_{\sigma} \otimes k \rightarrow R_{\tau_{1}} \otimes k$ are

$$
x \mapsto \lambda_{x} \lambda_{1}^{-1} u, z \mapsto \lambda_{z} \lambda_{2}^{-1} v, y \mapsto 0, w \mapsto 0
$$

and

$$
x \mapsto 0, z \mapsto 0, y \mapsto \lambda_{y} \lambda_{2}^{-1} v, w \mapsto \lambda_{w} \lambda_{1}^{-1} u
$$

respectively, and the two different maps $R_{\sigma} \otimes k \rightarrow R_{\tau_{2}} \otimes k$ are given by

$$
x \mapsto \lambda_{x} \mu_{1}^{-1} s, w \mapsto \lambda_{w} \mu_{2}^{-1} t, y \mapsto 0, z \mapsto 0
$$

and

$$
x \mapsto 0, w \mapsto 0, y \mapsto \lambda_{y} \mu_{2}^{-1} t, z \mapsto \lambda_{z} \mu_{1}^{-1} s
$$

respectively. Then, as a scheme, $\check{X}_{0}(B, \mathscr{P}, s)$ can be thought of as $\mathbb{P}^{1} \times$ $\mathbb{P}^{1}$ with $\{0\} \times \mathbb{P}^{1}$ and $\{\infty\} \times \mathbb{P}^{1}$ glued using $(u, v) \mapsto\left(\lambda_{w} \lambda_{x}^{-1} u, \lambda_{y} \lambda_{z}^{-1} v\right)$, and $\mathbb{P}^{1} \times\{0\}$ and $\mathbb{P}^{1} \times\{\infty\}$ glued using $(s, t) \mapsto\left(\lambda_{z}^{-1} \lambda_{x} s, \lambda_{y}^{-1} \lambda_{w} t\right)$, or equivalently, if $\lambda=\lambda_{x}^{-1} \lambda_{y}^{-1} \lambda_{z} \lambda_{w}$, we glue using $(u, v) \mapsto(\lambda u, v)$ and $(s, t) \mapsto\left(\lambda^{-1} s, t\right)$. Thus the gluing is not arbitrary, and as far as the underlying scheme is concerned, we only have a one-parameter family of gluings. For a more general gluing the ample line bundle of bidegree $(1,1)$ would not descend. As we will see in Example 2.36, many gluings do not even give a scheme but an algebraic space. Also, different choices of gluing may give the same underlying scheme, but a different choice of line bundle.

Example 2.6. If $B$ is obtained from a reflexive polytope $\Xi \subseteq M_{\mathbb{R}}$ as in Example 1.18 and $\mathscr{P}$ is the polyhedral decomposition whose elements are proper faces of $\Xi$, then we can describe $\check{X}_{0}(B, \mathscr{P}, 1)$ (where 1 denotes the trivial gluing data) as follows. The polytope $\Xi$ defines a projective toric variety $\left(\mathbb{P}_{\Xi}, \mathcal{O}_{\mathbb{P}_{\Xi}}(1)\right)$, where $\Xi$ is the Newton polytope of $\mathcal{O}_{\mathbb{P}_{\Xi}}(1)$. The interior point $0 \in \Xi$ corresponds to a section $s_{0} \in \Gamma\left(\mathbb{P}_{\Xi}, \mathcal{O}_{\mathbb{P}_{\Xi}}(1)\right)$ which vanishes precisely once on each toric divisor of $\mathbb{P}_{\Xi}$. The zero locus $s_{0}=0$ coincides with $\check{X}_{0}(B, \mathscr{P}, 1)$, with polarization induced by $\mathcal{O}_{\mathbb{P}_{\Xi}}(1)$. Different choices of gluing data will give possibly non-isomorphic schemes. 
In fact, in general this may give too many choices of gluings. An arbitrary gluing will give a scheme which cannot appear as the central fibre of a toric degeneration (see Definition 4.1), even locally. The only gluings we are interested in are those for which there is a special sort of open covering, which we shall describe in detail in the fan picture. In theory, one should develop the cone and fan pictures on completely equal footing, but this would greatly increase the size of this paper. Furthermore, the two pictures are related by a discrete Legendre transform, so for us it is not really worth the effort to develop both pictures in equal generality. So we move on to the fan picture.

2.2. The fan picture. For any rational polyhedral fan $\Sigma$, we will write $X(\Sigma)$ for the toric variety over Spec $\mathbb{Z}$ defined by $\Sigma$.

Definition 2.7. If $\sigma \in \mathscr{P}$, let $X_{\sigma}:=X\left(\Sigma_{\sigma}\right)$, where $\Sigma_{\sigma}$ is the fan in $\mathcal{Q}_{\sigma, \mathbb{R}}$ defined in Definition 1.35.

Note that if $\Sigma$ is a fan in $M_{\mathbb{R}}$, and $\tau \in \Sigma$ with $\Sigma(\tau)$ the quotient fan (Definition 1.37), then there is a canonical closed embedding $X(\Sigma(\tau)) \rightarrow$ $X(\Sigma)$. This can be defined as follows: given $\sigma \in \Sigma, \sigma$ determines an open subset $X(\sigma) \subseteq X(\Sigma)$ given by $X(\sigma)=\operatorname{Spec} \mathbb{Z}\left[\sigma^{\vee} \cap N\right]$, where

$$
\sigma^{\vee}=\left\{n \in N_{\mathbb{R}} \mid\langle n, m\rangle \geq 0 \quad \forall m \in \sigma\right\}
$$

as usual. If $\sigma \supseteq \tau$, then

$$
X((\sigma+\mathbb{R} \tau) / \mathbb{R} \tau)=\operatorname{Spec} \mathbb{Z}\left[((\sigma+\mathbb{R} \tau) / \mathbb{R} \tau)^{\vee} \cap N\right] .
$$

Here $(\sigma+\mathbb{R} \tau) / \mathbb{R} \tau$ is a cone in $M_{\mathbb{R}} / \mathbb{R} \tau$, and we identify the dual of this space with $(\mathbb{R} \tau)^{\perp} \subseteq N_{\mathbb{R}}$. The inclusion

$$
X((\sigma+\mathbb{R} \tau) / \mathbb{R} \tau) \longrightarrow X(\sigma)
$$

is determined by the ring map given by

$$
z^{m} \longmapsto \begin{cases}z^{m} & \text { if } m \in \sigma^{\vee} \cap(\mathbb{R} \tau)^{\perp}=((\sigma+\mathbb{R} \tau) / \mathbb{R} \tau)^{\vee} ; \\ 0 & \text { otherwise. }\end{cases}
$$

These inclusions patch on open sets and give a well-defined inclusion $X(\Sigma(\tau))$ in $X(\Sigma)$.

We can then define a contravariant functor $F: \underline{\operatorname{Cat}}(\mathscr{P}) \rightarrow \underline{\mathrm{Sch}} / \mathbb{Z}$ as follows. We set

$$
F(\tau):=X_{\tau}
$$

For $e \in \operatorname{Hom}(\tau, \sigma)$, we obtain as before Definition 1.37 a surjection $p_{e}: \mathcal{Q}_{\tau, \mathbb{R}} \rightarrow \mathcal{Q}_{\sigma, \mathbb{R}}$ which identifies $\Sigma_{\sigma}$ with $\Sigma_{\tau}\left(K_{e}\right)$, where $K_{e}$ is the cone of $\Sigma_{\tau}$ corresponding to $e$. This gives a well-defined inclusion map

$$
F(e): X_{\sigma} \longrightarrow X_{\tau} \text {. }
$$

Example 2.8. Let $B=\mathbb{R}^{2} /(\mathbb{Z}(1,2)+\mathbb{Z}(2,1))$, with polyhedral decomposition coming from the quotient of the following periodic decomposition of $\mathbb{R}^{2}$ : 


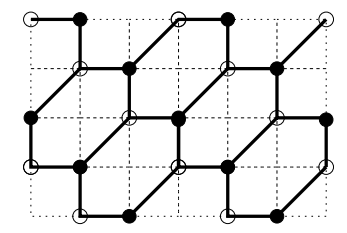

There are two vertices, $v_{1}$ and $v_{2}$, indicated by the two types of vertices in the figure. It is clear that $X_{v_{1}}$ and $X_{v_{2}}$ are both isomorphic to $\mathbb{P}^{2}$. There are three edges $\tau_{1}, \tau_{2}$ and $\tau_{3}$ with $X_{\tau_{i}}=\mathbb{P}^{1}$. The maps $X_{\tau_{i}} \rightarrow X_{v_{j}}$ identify $X_{\tau_{i}}$ with one of the various coordinate lines of $X_{v_{j}}$, as dictated by the combinatorics of the picture. There is one maximal cell $\sigma, X_{\sigma}=p t$, and three different maps $X_{\sigma} \rightarrow X_{v_{1}}$ and three different maps $X_{\sigma} \rightarrow X_{v_{2}}$, each identifying $X_{\sigma}$ with one of the three zero-dimensional strata of $X_{v_{i}}$.

We now proceed to twisting and gluing. Let $S$ be any scheme. We denote by $\mathbb{G}_{m}(S)$ the group of invertible regular functions on $S$.

Recalling the open covering $\mathscr{W}$ from Definition 1.25 , we denote by $H^{p}(\mathscr{W}, \cdot)$ the Čech cohomology groups with respect to the open covering $\mathscr{W}$. We write

$$
W_{\tau_{1} \cdots \tau_{p}}:=W_{\tau_{1}} \cap \cdots \cap W_{\tau_{p}} .
$$

Lemma 2.9. $W_{\tau_{1} \cdots \tau_{p}} \neq \emptyset$ if and only if $\tau_{1} \subseteq \cdots \subseteq \tau_{p}$ after reordering. Also, if $\tau \subsetneq \sigma$, then for each $e \in \operatorname{Hom}(\tau, \sigma), W_{\tau \sigma}$ contains a unique connected component intersecting e, which we write as $W_{e}$. This gives a one-to-one correspondence between components of $W_{\tau \sigma}$ and $\operatorname{Hom}(\tau, \sigma)$. More generally, the connected components of $W_{\tau_{1} \cdots \tau_{p}}$ are in one-to-one correspondence with $p-1$ dimensional simplices of $\operatorname{Bar}(\mathscr{P})$ with vertices at the barycenters of $\tau_{1}, \ldots, \tau_{p}$.

Proof. The first statement follows immediately from the fact that $W_{\tau_{1} \cdots \tau_{p}}$ is the union of the interiors of all simplices of $\operatorname{Bar}(\mathscr{P})$ containing the barycenters of $\tau_{1}, \ldots, \tau_{p}$. To prove the second statement, let $W_{e}$ be the union of the interiors of all simplices of $\operatorname{Bar}(\mathscr{P})$ containing $e$. It is easy to see each $W_{e}$ is connected, and an open subset of $B$. Furthermore, any two simplices of $\operatorname{Bar}(\mathscr{P})$ have disjoint interior, so $W_{\tau \sigma}=\coprod_{e \in \operatorname{Hom}(\tau, \sigma)} W_{e}$. Thus $\left\{W_{e} \mid e \in \operatorname{Hom}(\tau, \sigma)\right\}$ is the set of connected components of $W_{\tau \sigma}$. The general case is similar. q.e.d.

Definition 2.10. Let $S$ be a scheme. We call a Čech 1-cocycle $s=\left(s_{e}\right)_{e \in \amalg \operatorname{Hom}(\tau, \sigma)}$ for the sheaf $\mathcal{Q} \mathscr{P} \otimes \mathbb{G}_{m}(S)$ on $B$ with respect to the open covering $\mathscr{W}$ closed gluing data (for the fan picture) for $\mathscr{P}$ over $S$. Here $s_{e} \in \Gamma\left(W_{e}, \mathcal{Q}_{\mathscr{P}} \otimes \mathbb{G}_{m}(S)\right)=\mathcal{Q}_{\sigma} \otimes \mathbb{G}_{m}(S)$ for $e: \tau \rightarrow \sigma$.

We sometimes write $s_{\tau \sigma}$ instead of $s_{e}$ when $e: \tau \rightarrow \sigma$ is clear from the context. 
Since $\Sigma_{\sigma}$ is a fan living in $\mathcal{Q}_{\sigma, \mathbb{R}}, \mathcal{Q}_{\sigma} \otimes \mathbb{G}_{m}(S)$ acts on $X_{\sigma} \times S$, and hence $s_{e}$ gives an automorphism

$$
s_{e}: X_{\sigma} \times S \longrightarrow X_{\sigma} \times S .
$$

Thus we have

Definition 2.11. For closed gluing data $s$ for $\mathscr{P}$, we define a functor

$$
F_{S, s}: \underline{\operatorname{Cat}}(\mathscr{P}) \longrightarrow \underline{\mathrm{Sch}} / S
$$

by

$$
F_{S, s}(\tau):=X_{\tau} \times S
$$

and for $e \in \operatorname{Hom}(\tau, \sigma)$,

$$
F_{S, s}(e):=(F(e) \times i d) \circ s_{e}: X_{\sigma} \times S \longrightarrow X_{\tau} \times S .
$$

Let $X$ be an algebraic space, $A$ a closed subspace and $A \rightarrow \bar{A}$ a finite morphism. Then the fibred coproduct $X \amalg_{A} \bar{A}$ in the category of algebraic spaces is an algebraic space. This is a corollary of Artin's theory of the existence of formal modifications, see [2], Theorem 6.1. In particular, the identification of two algebraic spaces along a closed subspace is again an algebraic space. Applying this fact inductively it is possible to construct the direct limit

$$
X_{0}(B, \mathscr{P}, s)=\lim _{\longrightarrow} F_{S, s}
$$

as an algebraic space locally of finite type over $S$. In other words, for every $\tau \in \mathscr{P}$ there is a morphism $q_{\tau}: X_{\tau} \times S \rightarrow X_{0}(B, \mathscr{P}, s)$ and for every $e \in \operatorname{Hom}(\tau, \sigma)$ we have $q_{\sigma}=q_{\tau} \circ F_{S, s}(e)$; moreover, $X_{0}(B, \mathscr{P}, s)$ is a universal object in the category of algebraic spaces with this property. The constructed space is of finite type over $S$ whenever $\mathscr{P}$ is finite.

Instead of providing full details for the use of Artin's result, we are going to construct $X_{0}(B, \mathscr{P}, s)$ directly as a quotient of a scheme by an étale equivalence relation. In other words, instead of constructing $X_{0}(B, \mathscr{P}, s)$ by gluing proper toric varieties together along closed substrata, we glue together a collection of open sets to obtain $X_{0}(B, \mathscr{P}, s)$. However, this cannot be done for all choices of $s$ (see Remark 2.33), and in fact those choices of $s$ for which this cannot be done are irrelevant for our theory. The existence of an open covering of $X_{0}(B, \mathscr{P}, s)$ by some standard open sets is crucial. Thus we will only discuss the open gluings, and this topic occupies the rest of this section.

First let us explain what these standard open sets are.

Definition 2.12. Let $\sigma \in \mathscr{P}_{\max }$ be a maximal cell, $y \in \operatorname{Int}(\sigma)$ and $\tilde{\sigma} \subseteq \Lambda_{\mathbb{R}, y}$ as in Definition 2.1. We denote by $C(\sigma)^{\vee}$ the dual cone to $C(\sigma)$ in $\check{\Lambda}_{\mathbb{R}, y} \oplus \mathbb{R}$, and set

$$
P_{\sigma}=C(\sigma)^{\vee} \cap\left(\check{\Lambda}_{y} \oplus \mathbb{Z}\right),
$$


so that the affine toric variety defined by the fan of faces of $C(\sigma)$ is

$$
U(\sigma):=\operatorname{Spec} \mathbb{Z}\left[P_{\sigma}\right] .
$$

In addition, the projection $\Lambda_{v} \oplus \mathbb{Z} \rightarrow \mathbb{Z}$ defines an element $\rho_{\sigma} \in P_{\sigma} \subseteq$ $\check{\Lambda}_{v} \oplus \mathbb{Z}$, hence a monomial regular function $z^{\rho_{\sigma}}$ on $U(\sigma)$. Define $V(\sigma)$ to be the zero scheme of this regular function. Note that by construction $z^{\rho_{\sigma}}$ vanishes precisely once on each toric divisor of $U(\sigma)$, so $V(\sigma)$ is the reduced union of all toric divisors in $U(\sigma)$. By the uniqueness property of $\tilde{\sigma}$ as in Definition 2.1, $U(\sigma)$ and $V(\sigma)$ are unique up to unique toric isomorphism.

$V(\sigma)$ can be described in terms of a monoid ring also:

Definition 2.13. Let $\sigma$ be a (not necessarily strictly convex) cone in $N_{\mathbb{R}}$, for $N$ a lattice, and let $P$ be the monoid $\sigma \cap N$. We write

$$
\partial P:=(\partial \sigma \cap N) \cup\{\infty\},
$$

with addition

$$
p+q= \begin{cases}p+q & \text { if } p, q, p+q \in \partial \sigma \\ \infty & \text { otherwise }\end{cases}
$$

We define the monoid ring $\mathbb{Z}[\partial P]$ by using the convention that $z^{\infty}=0$.

It is easy to see that in the notation of Definition 2.12, $V(\sigma)=$ $\operatorname{Spec} \mathbb{Z}\left[\partial P_{\sigma}\right]$.

The collection $\left\{V(\sigma) \times S \mid \sigma \in \mathscr{P}_{\max }\right\}$ will serve as our étale cover of $X_{0}(B, \mathscr{P}, s)$. The next task is to write down the étale equivalence relation on $\coprod_{\sigma \in \mathscr{P}_{\max }} V(\sigma) \times S$. This will be a closed subspace

$$
\begin{aligned}
\mathfrak{R} \subset\left(\coprod_{\sigma_{1} \in \mathscr{P}_{\max }} V\left(\sigma_{1}\right) \times S\right) \times{ }_{S}\left(\coprod_{\sigma_{2} \in \mathscr{P}_{\max }} V\left(\sigma_{2}\right) \times S\right) \\
=\coprod_{\sigma_{1}, \sigma_{2} \in \mathscr{P}_{\max }} V\left(\sigma_{1}\right) \times V\left(\sigma_{2}\right) \times S .
\end{aligned}
$$

Remark 2.14. The guiding principle for the construction of this equivalence relation comes from the relation of the $V(\sigma)$ with the proper schemes $X_{v}$ from before. A vertex $v \in \tilde{\sigma}$ gives the $n$-dimensional face $(v, 1)^{\perp} \cap C(\sigma)^{\vee}$ of $C(\sigma)^{\vee}$. The integral points of this face form a monoid $P_{\sigma, v}$, and Spec $\mathbb{Z}\left[P_{\sigma, v}\right]$ is one of the irreducible components of $V(\sigma)$. An alternative way to see this component is as follows: for $y \in \operatorname{Int}(\sigma)$, identify $\Lambda_{y}$ with $\Lambda_{v}$ by parallel transport in $\sigma$ and project

$$
(v, 1)^{\perp} \cap C(\sigma)^{\vee} \subset\left(\Lambda_{\mathbb{R}, v} \oplus \mathbb{R}\right)^{*}=\check{\Lambda}_{\mathbb{R}, v} \oplus \mathbb{R}
$$

onto the first factor. This identifies $(v, 1)^{\perp} \cap C(\sigma)^{\vee}$ with the dual of the tangent wedge to $\tilde{\sigma}$ at $v$. Thus the irreducible component $\operatorname{Spec} \mathbb{Z}\left[P_{\sigma, v}\right]$ is naturally identified with the affine open set of the toric variety $X_{v}=$ $X\left(\Sigma_{v}\right)$ corresponding to the cone of $\Sigma_{v}$ determined by $v \in \tilde{\sigma}$. 
The problem in writing this down is that the gluing of open subsets for maximal cells $\sigma_{1}, \sigma_{2}$ intersecting in more than one point does not generally come from gluing of open sets in the ambient spaces $U\left(\sigma_{1}\right), U\left(\sigma_{2}\right)$. Rather each vertex in the intersection prescribes one gluing of an open subset of $U\left(\sigma_{i}\right)$; the restrictive form of the monodromy assures compatibility of the gluing only after restriction to the divisor $V\left(\sigma_{i}\right) \subset U\left(\sigma_{i}\right)$.

Construction 2.15. Let us first discuss the case of only two maximal cells $\sigma_{1}, \sigma_{2}$ without self-intersections and intersecting in one cell $\tau=\sigma_{1} \cap \sigma_{2}$. Also let us assume the gluing parameter $s$ to be trivial for the time being. Choose vertices $v_{i} \in \sigma_{i}$ and embeddings $\sigma_{i}=\tilde{\sigma}_{i} \subset \Lambda_{\mathbb{R}, v_{i}}$. We are going to use the notations

$$
\begin{aligned}
& M_{i}=\Lambda_{v_{i}}, \quad N_{i}=M_{i}^{*}, \quad P_{i}:=P_{\sigma_{i}}=C\left(\sigma_{i}\right)^{\vee} \cap\left(N_{i} \oplus \mathbb{Z}\right), \\
& \rho_{i}:=\rho_{\sigma_{i}}=(0,1) \in N_{i} \oplus \mathbb{Z} .
\end{aligned}
$$

For each vertex $w \in \tau$, there are linear identifications

$$
\psi_{w}: M_{2} \longrightarrow M_{1}, \quad \psi_{w}^{t}: N_{1} \longrightarrow N_{2}
$$

given by parallel transport from $v_{2}$ through $w$ to $v_{1}$. We will distinguish $\tau$ as a face of $\sigma_{1}$ or a face of $\sigma_{2}$ by using the notation $\tau_{i} \subset \sigma_{i}$.

We wish to identify open subsets $V\left(\tau_{i}\right) \subseteq V\left(\sigma_{i}\right)$ and a natural isomorphism between $V\left(\tau_{1}\right)$ and $V\left(\tau_{2}\right)$.

To begin, it is worth understanding $V\left(\sigma_{i}\right)$ a bit better. The monoid $\partial P_{i}$ has an interpretation in terms of fans. Let $\check{\Sigma}_{i}$ be the normal fan to $\sigma_{i}$, living in $N_{i} \otimes \mathbb{R}$. Then by projecting $N_{i} \oplus \mathbb{Z} \rightarrow N_{i}$, we can in fact identify $\partial P_{i}$ with the monoid $N_{i} \cup\{\infty\}$ with addition

$$
p+q= \begin{cases}p+q & \text { if } p, q \text { are in a common cone of } \check{\Sigma}_{i} \\ \infty & \text { otherwise. }\end{cases}
$$

Note that for any face $\omega \subseteq \sigma_{i}$, there is a corresponding face $\check{\omega}$ of $C\left(\sigma_{i}\right)^{\vee}$ dual to $C(\omega)$, i.e.,

$$
\check{\omega}=\left\{n \in C\left(\sigma_{i}\right)^{\vee} \mid\langle n,(m, 1)\rangle=0 \text { for all } m \in \omega\right\} .
$$

We will also denote by $\check{\omega}$ the projection of $\breve{\omega}$ to $N_{i}$; this is the cone of $\check{\Sigma}_{i}$ denoted $\check{K}_{\omega}$ in Definition 1.38. In particular, Spec $\mathbb{Z}\left[\check{\omega} \cap N_{i}\right]$ is then a closed subscheme of $V\left(\sigma_{i}\right)$, a toric stratum corresponding to $\omega$.

In future $\check{\omega}$ may refer both to the face of $C\left(\sigma_{i}\right)^{\vee} \subset\left(N_{i} \oplus \mathbb{Z}\right) \otimes \mathbb{R}$ and to its projection to $N_{i} \otimes \mathbb{R}$. It will always be clear from the context which alternative applies.

Now define

$$
U\left(\tau_{i}\right)=\operatorname{Spec} \mathbb{Z}\left[Q_{i}\right]
$$

with

$$
Q_{i}:=C\left(\tau_{i}\right)^{\vee} \cap\left(N_{i} \oplus \mathbb{Z}\right)=P_{i}+\left(\mathbb{R} \check{\tau}_{i} \cap\left(N_{i} \oplus \mathbb{Z}\right)\right)
$$


As an abstract toric variety $U\left(\tau_{i}\right)$ is isomorphic to

$$
\operatorname{Spec} \mathbb{Z}\left[\operatorname{Hom}\left(C\left(\tau_{i}\right) \cap\left(M_{i} \oplus \mathbb{Z}\right), \mathbb{N}\right)\right] \times \mathbb{G}_{m}^{\operatorname{codim} \tau_{i}}
$$

in particular, the isomorphism class as an abstract toric variety depends only on $\tau$. It is easy to see that $\mathbb{Z}\left[\partial Q_{i}\right] \cong \mathbb{Z}\left[Q_{i}\right] /\left(z^{\rho_{i}}\right)$. Thus in analogy with the definition for maximal cones we now define

$$
V\left(\tau_{i}\right)=\operatorname{Spec} \mathbb{Z}\left[\partial Q_{i}\right] .
$$

The inclusion $P_{i} \subseteq Q_{i}$ induces the inclusion $U\left(\tau_{i}\right) \subseteq U\left(\sigma_{i}\right)$, and this restricts to an inclusion $V\left(\tau_{i}\right) \subseteq V\left(\sigma_{i}\right)$.

It is worth underlining a very important point here. We were able to define $V(\sigma)$ for $\sigma$ maximal in a completely canonical way. On the other hand, for $\tau$ non-maximal, we have not defined $V(\tau)$ but only $V\left(\tau_{i}\right)$ as a subset of the canonically defined $V\left(\sigma_{i}\right)$. The point is that $V(\tau)$ is not well-defined up to a unique isomorphism because of holonomy in a neighbourhood of $\operatorname{Int}(\tau)$, i.e., there is no canonical affine structure on $\sigma_{1} \cup \sigma_{2}$. It is only when this holonomy is trivial and the affine structure of $B$ is defined in a neighbourhood of $\operatorname{Int}(\tau)$ that $V(\tau)$ becomes welldefined. It is this indeterminacy of $V(\tau)$ when there is holonomy which causes the gluing between $V\left(\tau_{1}\right)$ and $V\left(\tau_{2}\right)$ below to be non-trivial. Believing in the independent existence of $V(\tau)$ is an easy trap to fall into, one we have made countless times. In particular, there is no canonical isomorphism between $U\left(\tau_{1}\right) \subset U\left(\sigma_{1}\right)$ and $U\left(\tau_{2}\right) \subset U\left(\sigma_{2}\right)$, and we cannot glue these schemes, even though $V\left(\tau_{1}\right)$ and $V\left(\tau_{2}\right)$ will glue.

The monoid $\partial Q_{i}$ also has an interpretation in terms of fans as before. By projecting $\partial C\left(\tau_{i}\right)^{\vee}$ to $N_{i}$, we obtain an identification $\partial Q_{i}=N_{i} \cup\{\infty\}$ with addition

$$
p+q= \begin{cases}p+q & \text { if } p, q \text { are in a common cone of } \check{\tau}_{i}^{-1} \check{\Sigma}_{i} \\ \infty & \text { otherwise. }\end{cases}
$$

Here $\check{\tau}_{i}^{-1} \check{\Sigma}_{i}$ is the localisation of the fan $\check{\Sigma}_{i}$ at $\check{\tau}_{i}$ as defined in Definition 1.37.

Here is the key point of the construction. We want to identify the sets $V\left(\tau_{1}\right)$ and $V\left(\tau_{2}\right)$ in order to glue together $V\left(\sigma_{1}\right)$ and $V\left(\sigma_{2}\right)$. This is done by specifying an isomorphism of monoids

$$
\phi: \partial Q_{1} \longrightarrow \partial Q_{2} \text {. }
$$

Via the embedding $\partial Q_{i} \backslash\{\infty\} \subset N_{i}$ such a morphism is equivalent to a piecewise linear map $\phi: N_{1} \rightarrow N_{2}$ linear on cones of $\check{\tau}_{1}^{-1} \check{\Sigma}_{1}$, mapping $\check{\tau}_{1}^{-1} \check{\Sigma}_{1}$ to $\check{\tau}_{2}^{-1} \check{\Sigma}_{2}$. To define $\phi$ on the maximal cone in $\partial Q_{1}$ corresponding to a vertex $w \in \tau$ use the linear map ${ }^{t} \psi_{w}: N_{1} \rightarrow N_{2}$ defined by parallel transport through $w$ as above. Parallel transport identifies the $\sigma_{i}$ with maximal cells in the induced polyhedral decomposition of $R_{w} \subseteq \Lambda_{\mathbb{R}, w}$, whose intersection maps to $\tau$. Since $\check{\tau}_{i}^{-1} \check{\Sigma}_{i}$ depends only on $\tau_{i} \subset M_{i}$ this provides the desired identification of fans. 
We need to check that our definition of $\phi$ is well-defined on intersections of cones. Let $w_{1}, w_{2}$ be vertices of $\tau$ and $\check{w}_{i} \subset N_{1}$ the corresponding maximal cones of $\check{\tau}_{1}^{-1} \check{\Sigma}_{1}$. Then $\check{\omega}:=\check{w}_{1} \cap \check{w}_{2}$ is dual to a subface $\omega \subset \tau$ containing $w_{1}, w_{2}$. For $n \in \check{w}_{1} \cap \check{w}_{2}$ and $m \in M_{1}$ we compute

$$
\left\langle{ }^{t} \psi_{w_{2}}^{-1 t} \psi_{w_{1}}(n), m\right\rangle=\left\langle n, \psi_{w_{1}} \psi_{w_{2}}^{-1}(m)\right\rangle=\left\langle n, T_{\gamma}(m)\right\rangle,
$$

where $T_{\gamma}$ is parallel transport along the loop passing from $v_{1}$ to $w_{2}$ inside $\sigma_{1}$, then to $w_{1}$ inside $\sigma_{2}$ and finally back to $v_{1}$. By Proposition 1.29, $T_{\gamma}(m)$ is congruent to $m$ modulo the tangent space $\check{\omega}^{\perp}$ of $\omega$. Hence $\left\langle n, T_{\gamma}(m)\right\rangle=\langle n, m\rangle$ for all $m \in M_{1}$ and

$$
{ }^{t} \psi_{w_{2}}(n)={ }^{t} \psi_{w_{1}}(n)
$$

Thus $\phi$ is well-defined. This gives our gluing isomorphism

$$
\Phi_{\sigma_{1} \sigma_{2}}: V\left(\tau_{2}\right) \longrightarrow V\left(\tau_{1}\right) \text {. }
$$

Example 2.16. We illustrate the definition of $\phi$ using Example 1.16, (2). Let $\sigma_{1}$ and $\sigma_{2}$ denote the left and right triangles in Example $1.16,(2)$ respectively, in either the left or right-hand pictures, and let $\tau=\sigma_{1} \cap \sigma_{2}$. Then using the left-hand picture, $C\left(\sigma_{1}\right)^{\vee}$ is the cone in $N_{\mathbb{R}} \oplus \mathbb{R}$ generated by

$$
(-1,0,0),(0,1,0),(1,-1,1)
$$

and $C\left(\sigma_{2}\right)^{\vee}$ is the cone generated by

$$
(1,0,0),(0,1,0),(-1,-1,1) \text {. }
$$

In both instances $\check{\tau}_{i}$ is the cone generated by the first vector. Then $\check{\Sigma}_{1}$ and $\check{\Sigma}_{2}$ are
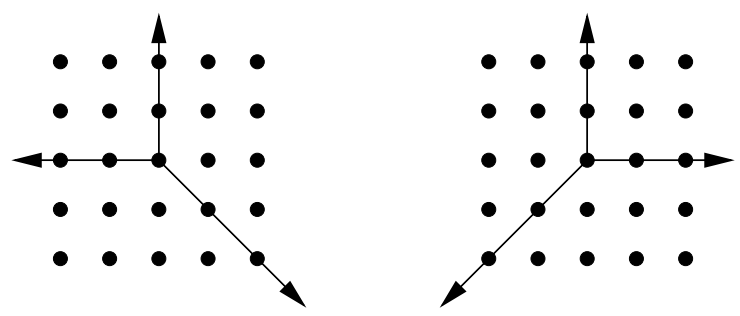

obtained by forgetting the last coordinate. Indeed, this is just the normal fan of $\sigma_{i}$. Finally, localizing at $\check{\tau}$ gives $\partial Q_{1}$ and $\partial Q_{2}$ determined by the picture

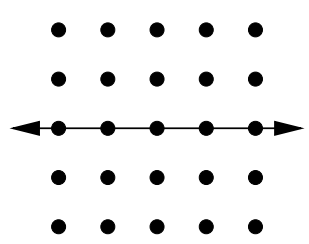

The upper half plane corresponds to the vertex $(0,0)$ and the lower halfplane corresponds to the vertex $(0,1)$. Now given that we have been using the left-hand chart in Example 1.16, (2), the map $\psi_{(0,0)}: M_{2} \rightarrow$ 
$M_{1}$ should be viewed as the identity, while $\psi_{(0,1)}: M_{2} \rightarrow M_{1}$ is given by the matrix $\left(\begin{array}{ll}1 & 0 \\ 1 & 1\end{array}\right)$. This is the transformation which takes $\sigma_{2}$ in the left-hand chart to $\sigma_{2}$ in the right-hand chart. Thus if we denote by $x$ the element $(1,0) \in \partial Q_{i}, y$ the element $(0,1), z$ the element $(0,-1)$, then

$$
\phi: \partial Q_{1} \longrightarrow \partial Q_{2}
$$

takes $x \mapsto x, y \mapsto y$ and $z \mapsto x^{-1} z$ since

$$
\psi_{(0,1)}^{t}\left(\begin{array}{c}
0 \\
-1
\end{array}\right)=\left(\begin{array}{l}
-1 \\
-1
\end{array}\right)=-\left(\begin{array}{l}
1 \\
0
\end{array}\right)+\left(\begin{array}{c}
0 \\
-1
\end{array}\right)
$$

The map $\phi$ induces the gluing map

$$
\Phi_{\sigma_{1} \sigma_{2}}: \operatorname{Spec} \mathbb{Z}\left[x, x^{-1}, y, z\right] /(y z) \longrightarrow \operatorname{Spec} \mathbb{Z}\left[x, x^{-1}, y, z\right] /(y z)
$$

Example 2.17. Next we do a three-dimensional example. Here again we use two different cuts, and the shaded area shows where we make cuts.
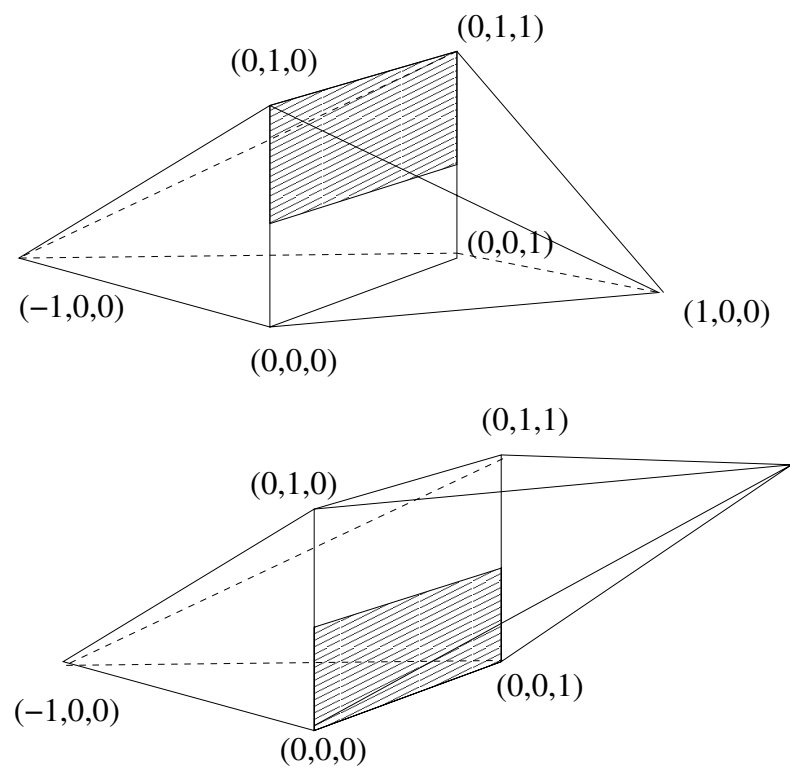

$(1,1,0)$

This time we just draw the fan $\check{\Sigma}_{i}\left(\check{\tau}_{i}\right)$ (the fan $\check{\tau}_{i}^{-1} \check{\Sigma}_{i}$ is the pullback of this to $\left.M_{i}\right)$ living in $M_{i} /(1,0,0) \mathbb{Z}$ : 


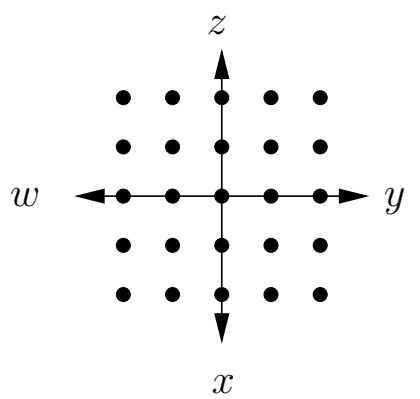

with the first through fourth quadrants corresponding to the vertices $(0,0,0),(0,1,0),(0,1,1)$ and $(0,0,1)$ of $\tau$ respectively. Then we can write

$$
\mathbb{Z}\left[\partial Q_{i}\right]=\mathbb{Z}\left[u, u^{-1}, x, y, z, w\right] /(y w, x z),
$$

where $x, y, z, w$ are primitive generators of the rays as labelled in the diagram and $u$ corresponds to $(1,0,0) \in M_{i}$. We take the maps $\psi_{(0,0,0)}$ and $\psi_{(0,0,1)}$ to be the identity, and then $\psi_{(0,1,0)}=\psi_{(0,1,1)}$ is given by the matrix

$$
\left(\begin{array}{lll}
1 & 0 & 0 \\
1 & 1 & 0 \\
0 & 0 & 1
\end{array}\right) .
$$

The gluing map $\phi: \mathbb{Z}\left[\partial Q_{1}\right] \rightarrow \mathbb{Z}\left[\partial Q_{2}\right]$ is

$$
u \mapsto u, x \mapsto x, y \mapsto y, z \mapsto z, w \mapsto u^{-1} w .
$$

Let us next discuss twisted gluings. Let $S$ be an arbitrary scheme, and we wish to glue the $V\left(\sigma_{i}\right) \times S$ along $V\left(\tau_{i}\right) \times S$ using a twisting of $\Phi_{\sigma_{1} \sigma_{2}} \times \operatorname{id}_{S}$. Twistings are given as follows. Let

$$
s_{i}: \partial Q_{i} \backslash\{\infty\} \longrightarrow \mathbb{G}_{m}(S)
$$

be a map satisfying

$$
s_{i}(p+q)=s_{i}(p) \cdot s_{i}(q) \text { if } p+q \neq \infty .
$$

We can view this as a piecewise multiplicative function on $N_{i}$. Then $s_{i}$ defines a map $s_{i}: V\left(\tau_{i}\right) \times S \rightarrow V\left(\tau_{i}\right) \times S$ induced by

$$
z^{p} \longmapsto s_{i}(p) z^{p} \text {. }
$$

We can then glue $V\left(\tau_{1}\right) \times S$ and $V\left(\tau_{2}\right) \times S$ via $s_{1}^{-1} \circ\left(\Phi_{\sigma_{1} \sigma_{2}} \times \operatorname{id}_{S}\right) \circ s_{2}$.

So far the discussion was essentially local, focusing on only two maximal cells intersecting in one common face. In general the part $\mathfrak{R}_{\sigma_{1} \sigma_{2}}$ of the equivalence relation in $V\left(\sigma_{2}\right) \times V\left(\sigma_{1}\right)$ may have more than one component. For any $\sigma \in \mathscr{P}_{\max }$, and a vertex $v \in \sigma$, we have $R_{v} \subseteq \Lambda_{\mathbb{R}, v}$, $\mathscr{P}_{v}$ and $\exp _{v}$ as usual, and $\tilde{\sigma} \in \mathscr{P}_{v}$ mapping to $\sigma$ via $\exp _{v}$. Denoting this map $\pi: \tilde{\sigma} \rightarrow \sigma$, it is an integral affine isomorphism in the interior of $\tilde{\sigma}$ and is finite-to-one on the boundary. This map is unique up to integral affine isomorphisms between different choices of $\tilde{\sigma}$. 
Now given $\sigma_{1}, \sigma_{2} \in \mathscr{P}_{\max }$, let $\tilde{\sigma}_{i} \subseteq M_{i} \otimes \mathbb{R}, M_{i}=\Lambda_{\sigma_{i}}, \pi_{i}: \tilde{\sigma}_{i} \rightarrow \sigma_{i}$ be as above. Consider the fibred product $\tilde{\sigma}_{1} \times_{B} \tilde{\sigma}_{2}$. Each connected component of this space will give one component of $\Re_{\sigma_{1} \sigma_{2}}$.

Lemma 2.18. Let $\sigma_{1}, \sigma_{2} \in \mathscr{P}_{\max }$. Then the connected components of $\tilde{\sigma}_{1} \times_{B} \tilde{\sigma}_{2}$ are graphs of integral affine isomorphisms $\tilde{\tau}_{1} \rightarrow \tilde{\tau}_{2}$ over $B$ for faces $\tilde{\tau}_{i} \subset \tilde{\sigma}_{i}$. In particular, the $\tilde{\tau}_{i}$ cover the same $\tau \subset \sigma_{1} \cap \sigma_{2}$. Exactly those isomorphisms occur that do not extend to larger subsets of $\tilde{\sigma}_{i}$.

Proof. We have disjoint decompositions $\tilde{\sigma}_{i}=\coprod_{\tilde{\tau} \subset \tilde{\sigma}_{i}} \operatorname{Int}(\tilde{\tau})$. By the definition of polyhedral decompositions $\left.\pi_{i}\right|_{\operatorname{Int}(\tilde{\tau})}$ is a homeomorphism onto its image. For any pair $\tilde{\tau}_{1}, \tilde{\tau}_{2}$ covering the same $\tau \subset \sigma_{1} \cap \sigma_{2}$ the composition $\left.\left(\left.\pi_{2}\right|_{\operatorname{Int}\left(\tilde{\tau}_{2}\right)}\right)^{-1} \circ \pi_{1}\right|_{\operatorname{Int}\left(\tilde{\tau}_{1}\right)}$ is affine. Hence it extends to an affine linear map $\lambda$ from $\mathbb{R} \tilde{\tau}_{1} \subset M_{1} \otimes \mathbb{R}$ to $\mathbb{R} \tilde{\tau}_{2} \subset M_{2} \otimes \mathbb{R}$. This shows already that $\tilde{\sigma}_{1} \times_{B} \tilde{\sigma}_{2}$ has a decomposition into polyhedral subsets that are graphs of affine transformations $\lambda: \tilde{\tau}_{1} \rightarrow \tilde{\tau}_{2}$ commuting with $\pi_{i}$.

To see how the strata fit together choose a vertex $w_{1} \in \tilde{\tau}_{1}$ and put $w_{2}=\lambda\left(w_{1}\right)$. Let $R_{w} \subseteq \Lambda_{\mathbb{R}, w}, \mathscr{P}_{w}, \exp _{w}$ be a chart for $(B, \mathscr{P})$ at $w=\pi_{1}\left(w_{1}\right)=\pi_{2}\left(w_{2}\right)$. By the definition of polyhedral decomposition there exist unique affine embeddings

$$
\tilde{\sigma}_{i} \longrightarrow R_{w}
$$

respecting the polyhedral decompositions and mapping $w_{i}$ to the origin. This induces integral affine maps $M_{i} \otimes \mathbb{R} \rightarrow \Lambda_{\mathbb{R}, w}$ for $i=1,2$, and the composition

$$
M_{1} \otimes \mathbb{R} \longrightarrow \Lambda_{\mathbb{R}, w} \longrightarrow M_{2} \otimes \mathbb{R}
$$

restricts to $\lambda$ on $\tilde{\tau}_{1}$. This shows that $\tilde{\sigma}_{1} \times_{B} \tilde{\sigma}_{2}$ has a decomposition as a union of polyhedra with each maximal cell the graph of an integral affine transformation $\lambda: \tilde{\tau}_{1} \rightarrow \tilde{\tau}_{2}$ that commutes with $\pi_{i}$ and does not extend to a map between larger faces of $\tilde{\sigma}_{i}$. Moreover, we see that locally $\tilde{\sigma}_{1} \times_{B} \tilde{\sigma}_{2}$ is isomorphic to the intersection of two (possibly equal) cells of the polyhedral decomposition of $R_{w}$. Hence the maximal cells in the polyhedral decomposition of $\tilde{\sigma}_{1} \times_{B} \tilde{\sigma}_{2}$ are disjoint. Therefore we have the claimed decomposition of $\tilde{\sigma}_{1} \times_{B} \tilde{\sigma}_{2}$ into pairwise disjoint polyhedra.

q.e.d.

Remark 2.19. The reader shy of algebraic spaces may read the remainder of this section with the restriction on $\mathscr{P}$ that the maps $\pi$ : $\tilde{\tau} \rightarrow \tau \subseteq B$ are injective for all $\tau \in \mathscr{P}$. It is then the case that the algebraic space constructed below only involves gluings of affine schemes along Zariski open subsets rather than étale open subsets, and hence produces a scheme.

Let $\tilde{\tau}_{1} \rightarrow \tilde{\tau}_{2}$ be an integral affine transformation whose graph is a connected component of $\tilde{\sigma}_{1} \times_{B} \tilde{\sigma}_{2}$, as established by the lemma. Except for the trivial case $\tilde{\tau}_{1}=\tilde{\sigma}_{1}=\tilde{\sigma}_{2}=\tilde{\tau}_{2}$ there is a unique edge of 
the barycentric subdivision of $\tilde{\sigma}_{i}$ joining the barycenter of $\tilde{\tau}_{i}$ with the barycenter of $\tilde{\sigma}_{i}$. This edge determines $\tilde{\tau}_{i}$ uniquely. It also corresponds uniquely to an edge of the barycentric subdivision of $\sigma_{i}$. We may thus index the components of $\tilde{\sigma}_{1} \times_{B} \tilde{\sigma}_{2}$ by $\left(e_{1}, e_{2}\right)$ with $e_{i} \in \operatorname{Hom}\left(\tau, \sigma_{i}\right)$ and $\tau \subset \sigma_{1} \cap \sigma_{2}$. The mentioned trivial case is covered by (id,id). Not all pairs occur, but only those that are maximal for the partial order defined by

$$
\left(e_{1}, e_{2}\right)<\left(f_{1}, f_{2}\right) \quad \Longleftrightarrow \quad \exists h \in \operatorname{Hom}(\tau, \omega): e_{i}=f_{i} \circ h .
$$

Example 2.20. Consider $B=\mathbb{R}^{2} / \mathbb{Z}^{2}$ with the affine structure induced from $\mathbb{R}^{2}$, and the polyhedral decomposition with one maximal cell $\sigma$ covered by $\tilde{\sigma}=[0,1] \times[0,1] \subset \mathbb{R}^{2}$. The following figure shows the fibred product of $\tilde{\sigma}$ with itself over $B$.

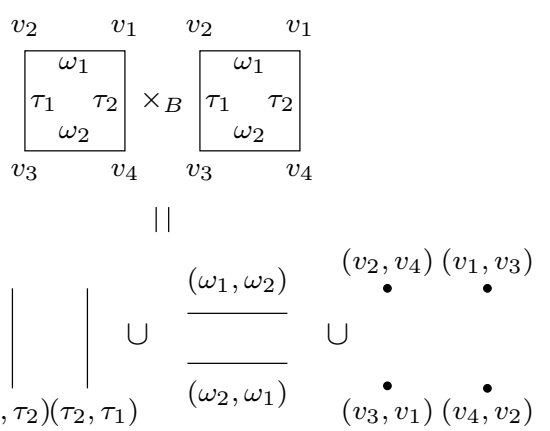

There are $4 \cdot 4=16$ vertices in $\tilde{\sigma} \times_{B} \tilde{\sigma}$; a pair of vertices $\left(v_{i}, v_{j}\right)$ is isolated in $\tilde{\sigma} \times_{B} \tilde{\sigma}$ if $v_{i}$ and $v_{j}$ do not belong to a proper face of $\tilde{\sigma}$. This is the case if $i-j \equiv 2$ modulo 4 . Similarly, the pairs $\left(\tau_{i}, \tau_{i}\right)$ and $\left(\omega_{i}, \omega_{i}\right)$ are not maximal: the identity isomorphisms $\mathrm{id}_{\tau_{i}}$ and $\mathrm{id}_{\omega_{i}}$ extend to the identity on $\tilde{\sigma}$.

Definition 2.21. We have thus indexed the connected components of $\tilde{\sigma}_{1} \times_{B} \tilde{\sigma}_{2}$ by a subset of pairs $\left(e_{1}, e_{2}\right) \in \operatorname{Hom}\left(\tau, \sigma_{1}\right) \times \operatorname{Hom}\left(\tau, \sigma_{2}\right)$ for $\tau \subset \sigma_{1} \cap \sigma_{2}$. By Lemma 2.18 it makes sense to call pairs corresponding to connected components maximal.

We will need the following:

Definition 2.22. Let $e: \tau \rightarrow \sigma$. Then $e$ determines a cone $K_{e}$ in $\Sigma_{\tau}$, and hence determines an open subset $V_{e} \subseteq X_{\tau}$ with $V_{e}=\operatorname{Spec} \mathbb{Z}\left[K_{e}^{\vee} \cap\right.$ $\left.\mathcal{Q}_{\tau}^{*}\right]$. The embedding $V_{e} \subseteq X_{\tau}$ is canonical.

For any maximal pair $\left(e_{1}, e_{2}\right)$ let $\tilde{\tau}_{i} \subseteq \tilde{\sigma}_{i}$ be the face given by $e_{i}: \tau \rightarrow$ $\sigma_{i}$. For any common choice of vertex $f: w \rightarrow \tau$ of $\tilde{\tau}_{i}$ the compositions $e_{i} \circ f$ define a path joining the interior of $\sigma_{2}$ to the interior of $\sigma_{1}$ passing through $w$. Parallel transport along this path defines an affine map $\psi_{w}: \Lambda_{\sigma_{2}} \rightarrow \Lambda_{\sigma_{1}}$; note this depends on $\left(e_{1}, e_{2}\right)$ and not just $\sigma_{1}, \sigma_{2}$ and $w$. As in the case covered in Construction 2.15 without self-intersections this induces an isomorphism between the irreducible components $V_{e_{1} \circ f} \cap$ 
$V\left(\tau_{1}\right)$ and $V_{e_{2} \circ f} \cap V\left(\tau_{2}\right)$ of $V\left(\tau_{1}\right)$ and $V\left(\tau_{2}\right)$. The monodromy argument as in Construction 2.15 shows that these isomorphisms are compatible on intersections. We obtain an isomorphism

$$
\Phi_{e_{1} e_{2}}: V\left(\tau_{2}\right) \longrightarrow V\left(\tau_{1}\right),
$$

of the open subschemes $V\left(\tau_{i}\right) \subset V\left(\sigma_{i}\right)$, defined above, which is toric on each irreducible component.

Construction 2.23. We next want to twist the isomorphisms $\Phi_{e_{1} e_{2}}$ over an arbitrary scheme $S$. We need to describe automorphisms of the $V(\tau) \times S$ slightly more abstractly. Let $\tau \in \mathscr{P}$ be any cell, and let $\tilde{\tau} \subseteq \Lambda_{\tau, \mathbb{R}}$ be as in Definition 1.38, with $\pi: \tilde{\tau} \rightarrow \tau$ the map to $B$.

Consider the sheaf $\pi^{*}\left(\mathcal{Q}_{\mathscr{P}} \otimes \mathbb{G}_{m}(S)\right)$. This can be viewed as follows. If we choose any $\sigma \in \mathscr{P}_{\max }$ with $e: \tau \rightarrow \sigma$ a morphism, then we can identify $\tilde{\tau}$ with a face of $\tilde{\sigma} \subseteq \Lambda_{\sigma, \mathbb{R}}=\Lambda_{\mathbb{R}, z}$ for any $z \in \operatorname{Int}(\sigma)$. Under this identification, at a point $y \in \operatorname{Int}(\tilde{\omega})$, for $\tilde{\omega} \subseteq \tilde{\tau}$ a face, the stalk of $\pi^{*}\left(\mathcal{Q}_{\mathscr{P}} \otimes \mathbb{G}_{m}(S)\right)$ at $y$ is

$$
\left(\Lambda_{z} / \Lambda_{\omega}\right) \otimes \mathbb{G}_{m}(S)=\operatorname{Hom}\left(\Lambda_{\omega}^{\perp}, \mathbb{G}_{m}(S)\right) .
$$

Thus an element $s \in \Gamma\left(\tilde{\tau}, \pi^{*}\left(\mathcal{Q}_{\mathscr{P}} \otimes \mathbb{G}_{m}(S)\right)\right)$ can be viewed as given by a collection $\left(s_{v}\right), v$ ranging over the vertices of $\tilde{\tau}$, with $s_{v} \in \operatorname{Hom}\left(\check{\Lambda}_{z}\right.$, $\mathbb{G}_{m}(S)$ ), such that for any face $\tilde{\omega} \subseteq \tilde{\tau}$ containing vertices $v$ and $w$,

$$
\left.s_{v}\right|_{\Lambda_{\omega}^{\perp}}=\left.s_{w}\right|_{\Lambda_{\omega}^{\perp}}
$$

Now consider the fan $\check{\Sigma}_{\tau}$ in $\Lambda_{\tau, \mathbb{R}}^{*}$; by parallel transport to $z \in \operatorname{Int}(\sigma)$, we can consider $\Lambda_{\tau, \mathbb{R}}^{*}$ as a quotient of $\check{\Lambda}_{\mathbb{R}, z}$, and we can pull back the fan $\check{\Sigma}_{\tau}$ to $\check{\Lambda}_{\mathbb{R}, z}$ to get a fan of (not strictly) convex cones. We call this fan $\check{\tau}^{-1} \check{\Sigma}_{\sigma}$ as in Construction 2.15; it of course depends on $e$. The maximal cones of this fan are in one-to-one correspondence with vertices $v$ of $\tilde{\tau}$. Then $s$ defines a map $s: \check{\Lambda}_{z} \rightarrow \mathbb{G}_{m}(S)$ by defining $s$ to be $s_{v}$ on the cone of $\check{\tau}^{-1} \check{\Sigma}_{\sigma}$ corresponding to $v \in \tilde{\tau}$. This is a piecewise multiplicative function, and it is clear the set of piecewise multiplicative functions with respect to the fan $\check{\tau}^{-1} \check{\Sigma}_{\sigma}$ coincides with $\Gamma\left(\tilde{\tau}, \pi^{*}\left(\mathcal{Q}_{\mathscr{P}} \otimes \mathbb{G}_{m}(S)\right)\right)$. We then have $V(\tau) \subseteq V(\sigma)$ as usual, and $s$ then induces an automorphism of $V(\tau) \times S$, which we also denote by $s$.

Note that the group $\Gamma\left(\tilde{\tau}, \pi^{*}\left(\mathcal{Q}_{\mathscr{P}} \otimes \mathbb{G}_{m}(S)\right)\right)$ does not depend on $e: \tau \rightarrow \sigma$, so we only need to understand the dependence of the action on $V(\tau) \times S$ described above. Let $e_{i}: \tau \rightarrow \sigma_{i}, i=1,2$ with $\sigma_{i} \in \mathscr{P}_{\max }$. Then as usual, we obtain $V\left(\tau_{1}\right) \subseteq V\left(\sigma_{1}\right), V\left(\tau_{2}\right) \subseteq V\left(\sigma_{2}\right)$ and a map

$$
\Phi_{e_{1} e_{2}}: V\left(\tau_{2}\right) \longrightarrow V\left(\tau_{1}\right),
$$

(even if the pair $\left(e_{1}, e_{2}\right)$ is not maximal). Using the notation of Construction 2.15, the map $\Phi_{e_{1} e_{2}}$ is induced by a map of monoids

$$
\phi: \partial Q_{1} \longrightarrow \partial Q_{2}
$$


obtained on each cone corresponding to a vertex of $\tau_{i}$ by parallel translation through that vertex. Now $s \in \Gamma\left(\tilde{\tau}, \pi^{*}\left(\mathcal{Q} \mathscr{P} \otimes \mathbb{G}_{m}(S)\right)\right)$ defines both $s_{1}: \partial Q_{1} \rightarrow \mathbb{G}_{m}(S)$ and $s_{2}: \partial Q_{2} \rightarrow \mathbb{G}_{m}(S)$; as this has been done by parallel transport into the interiors of $\sigma_{1}$ and $\sigma_{2}$, it is easy to see that

$$
s_{1}(p)=s_{2}(\phi(p))
$$

and hence we have

$$
s_{1} \circ\left(\Phi_{e_{1} e_{2}} \times \operatorname{id}_{S}\right)=\left(\Phi_{e_{1} e_{2}} \times \operatorname{id}_{S}\right) \circ s_{2} .
$$

Thus, even though we have not defined $V(\tau)$ abstractly, the action of $\Gamma\left(\tilde{\tau}, \pi^{*}\left(\mathcal{Q}_{\mathscr{P}} \otimes \mathbb{G}_{m}(S)\right)\right)$ on $V(\tau)$ is well-defined with respect to the gluing maps $\Phi_{e_{1} e_{2}} \times \operatorname{id}_{S}$.

Definition 2.24. For $\tau \in \mathscr{P}$, we will write $P \check{P}(\tau)$ for the group $\Gamma\left(\tilde{\tau}, \pi^{*}\left(\mathcal{Q}_{\mathscr{P}} \otimes \mathbb{G}_{m}(S)\right)\right)$ described above. Here, $P M$ stands for piecewise multiplicative.

We can now define open gluing data.

Definition 2.25. Open gluing data for $\mathscr{P}$ over $S$ are data $s=$ $\left(s_{e}\right)_{e \in \amalg \operatorname{Hom}(\tau, \sigma)}$ with

$$
s_{e} \in \check{P M}(\tau)
$$

for $e: \tau \rightarrow \sigma$. They must satisfy

1) $s_{i d}=1$ for $i d: \sigma \rightarrow \sigma$ the identity.

2) For $e_{1}: \sigma_{1} \rightarrow \sigma_{2}, e_{2}: \sigma_{2} \rightarrow \sigma_{3}$ and $e_{3}=e_{2} \circ e_{1}$, yielding $\tilde{\sigma}_{1} \subseteq \tilde{\sigma}_{2} \subseteq \tilde{\sigma}_{3}$, we have

$$
s_{e_{2}} \mid \tilde{\sigma}_{1} \cdot s_{e_{1}}=s_{e_{3}} .
$$

Let $Z^{1}\left(\mathscr{P}, \mathcal{Q} \mathscr{P} \otimes \mathbb{G}_{m}(S)\right)$ denote the set of all open gluing data for $\mathscr{P}$ over $S$. This forms a group under multiplication.

We define trivial open gluing data to be open gluing data $s=\left(s_{e}\right)$ such that there exists $t=\left(t_{\sigma}\right)_{\sigma \in \mathscr{P}}$ with $t_{\sigma} \in P \breve{M}(\sigma)=\Gamma\left(\tilde{\sigma}, \pi^{*}\left(\mathcal{Q}_{\mathscr{P}} \otimes\right.\right.$ $\left.\left.\mathbb{G}_{m}(S)\right)\right)$ such that for any $e: \tau \rightarrow \sigma$,

$$
s_{e}=\left.t_{\tau}^{-1} t_{\sigma}\right|_{\tau}
$$

Here restriction denotes the restriction of $t_{\sigma}$ to the face $\tilde{\tau}$ of $\tilde{\sigma}$ corresponding to $e$.

We call the set of trivial open gluing data $B^{1}\left(\mathscr{P}, \mathcal{Q}_{\mathscr{P}} \otimes \mathbb{G}_{m}(S)\right)$, and write

$$
H^{1}\left(\mathscr{P}, \mathcal{Q}_{\mathscr{P}} \otimes \mathbb{G}_{m}(S)\right)=\frac{Z^{1}\left(\mathscr{P}, \mathcal{Q}_{\mathscr{P}} \otimes \mathbb{G}_{m}(S)\right)}{B^{1}\left(\mathscr{P}, \mathcal{Q}_{\mathscr{P}} \otimes \mathbb{G}_{m}(S)\right)} .
$$

Given open gluing data $s=\left(s_{e}\right)$, we can associate to it closed gluing data $\bar{s}=\left(\bar{s}_{e}\right)$ as follows. By the construction of $\mathcal{Q}_{\mathscr{P}}$, we have $\left(\mathcal{Q}_{\mathscr{P}}\right)_{y} \cong$ 
$\mathcal{Q}_{\tau} \cong \Gamma\left(W_{\tau}, \mathcal{Q}_{\mathscr{P}}\right)$ for $y \in \operatorname{Int}(\tau), \tau \in \mathscr{P}$. Thus for any $e: \tau \rightarrow \sigma$ there is a map given by the composition of natural maps

$$
\begin{aligned}
\Gamma\left(\tilde{\tau}, \pi^{*}\left(\mathcal{Q}_{\mathscr{P}} \otimes\right.\right. & \left.\left.\mathbb{G}_{m}(S)\right)\right) \longrightarrow\left(\mathcal{Q}_{\mathscr{P}}\right)_{y} \otimes \mathbb{G}_{m}(S) \\
& \stackrel{\cong}{\longrightarrow} \Gamma\left(W_{\tau}, \mathcal{Q}_{\mathscr{P}} \otimes \mathbb{G}_{m}(S)\right) \longrightarrow \Gamma\left(W_{e}, \mathcal{Q}_{\mathscr{P}} \otimes \mathbb{G}_{m}(S)\right),
\end{aligned}
$$

where the last map is restriction. We set $\bar{s}_{e}$ to be the image of $s_{e}$ under this map. This gives a Čech 1-cocycle, because of the 1-cocycle condition on open gluing data.

For a discussion of the difference between open and closed gluing data see Remark 2.33. The notation as a cohomology group is motivated by the fact that the definition fits into the framework of barycentric complexes developed in the appendix, however for the dual cell complex.

Given open gluing data $s$, we can define, for each maximal pair $\left(e_{1}, e_{2}\right)$,

$$
\Phi_{e_{1} e_{2}}(s):=s_{e_{1}}^{-1} \circ\left(\Phi_{e_{1} e_{2}} \times \mathrm{id}_{S}\right) \circ s_{e_{2}} .
$$

Define $\mathfrak{R}_{e_{1} e_{2}} \subset V\left(\sigma_{2}\right) \times V\left(\sigma_{1}\right) \times S$ as the graph of $\Phi_{e_{1} e_{2}}(s)$ relative $S$.

Example 2.26. The simplest example is the case $B=\mathbb{R} / \mathbb{Z}$, and $\mathscr{P}$ consists of one maximal cell $\sigma$ and one vertex $v$. Identifying $\tilde{\sigma}$ with the interval $[0,1], \tilde{\sigma} \times_{B} \tilde{\sigma}$ has three connected components: the diagonal and the points $(0,1)$ and $(1,0)$. In this case $V(\sigma) \cong \operatorname{Spec} \mathbb{Z}[x, y] /(x y)$, and if there is no twisting, the three corresponding components of $\mathfrak{R}$ in $V(\sigma) \times V(\sigma)=\operatorname{Spec} \mathbb{Z}\left[x, y, x^{\prime}, y^{\prime}\right] /\left(x y, x^{\prime} y^{\prime}\right)$ are the diagonal, the subvariety given by the equation $x^{\prime} y=1$, and the subvariety given by the equation $x y^{\prime}=1$. The quotient of $V(\sigma)$ by this étale equivalence relation is a nodal elliptic curve. One can also take $B=\mathbb{R} / n \mathbb{Z}$ with $n>1$ and subdivide $B$ into $n$ intervals of length one. In this case one obtains $n$ copies of Spec $\mathbb{Z}[x, y] /(x y)$, and after quotienting one obtains a cycle of $n$ rational curves, i.e., a Kodaira type $I_{n}$ fibre.

Lemma 2.27. The $\mathfrak{R}_{e_{1} e_{2}}$ are pairwise disjoint; their union

$$
\mathfrak{R}=\bigcup_{\left(e_{1}, e_{2}\right)} \mathfrak{R}_{e_{1} e_{2}}
$$

defines an étale, quasi-compact equivalence relation on $\coprod_{\sigma \in \mathscr{P}_{\max }} V(\sigma) \times$ $S$. Furthermore, $\mathfrak{R}$ is closed in $\coprod_{\sigma_{1}, \sigma_{2} \in \mathscr{P}_{\max }} V\left(\sigma_{1}\right) \times V\left(\sigma_{2}\right) \times S$.

Proof. By construction $\mathfrak{R}_{e_{1} e_{2}}$ is the graph of an isomorphism between open subsets of $V\left(\sigma_{1}\right) \times S$ and $V\left(\sigma_{2}\right) \times S$, and thus the projections of $\mathfrak{R}_{e_{1} e_{2}}$ to $V\left(\sigma_{i}\right) \times S$ are local isomorphisms, hence étale. Closedness of $\mathfrak{R}_{e_{1} e_{2}} \subseteq V\left(\sigma_{2}\right) \times V\left(\sigma_{1}\right) \times S$ follows from maximality of the pair $\left(e_{1}, e_{2}\right)$, so that the isomorphism cannot be extended. Quasicompactness holds by local finiteness of $\mathscr{P}$. 
To check that the $\mathfrak{R}_{e_{1} e_{2}}$ are pairwise disjoint it suffices to show this after pull-back to the irreducible components

$$
\left(V\left(\sigma_{2}\right) \cap X_{w_{2}}\right) \times\left(V\left(\sigma_{1}\right) \cap X_{w_{1}}\right) \times S,
$$

where the $w_{i} \in \tilde{\sigma}_{i}$ run over the set of vertices. (The notation $V\left(\sigma_{i}\right) \cap X_{w_{i}}$ is a slight abuse of notation: by this we mean the irreducible component of $V\left(\sigma_{i}\right)$ corresponding to the vertex $w_{i}$.) But by the local description of $\tilde{\sigma}_{1} \times_{B} \tilde{\sigma}_{2}$ a pair of vertices $\left(w_{1}, w_{2}\right)$ belongs to at most one maximal cell, indexed by $\left(e_{1}, e_{2}\right)$. This translates into the statement that only $\mathfrak{R}_{e_{1} e_{2}}$ intersects the irreducible component in question non-trivially.

By construction $\mathfrak{R}$ is symmetric and contains the diagonal. It remains to show transitivity. Let $\sigma_{1}, \sigma_{2}, \sigma_{3} \in \mathscr{P}_{\max }$ with $\pi_{i}: \tilde{\sigma}_{i} \rightarrow \sigma_{i}$ as before. It suffices to verify the transitivity relation on each irreducible component of $V\left(\sigma_{1}\right)$ separately. Let $w_{1} \in \tilde{\sigma}_{1}$ be a vertex and $\Re_{e_{2} e_{1}}$ a component of $\mathfrak{R}$ that is non-trivial on $V\left(\sigma_{1}\right) \cap X_{w_{1}}$. Let $R_{w} \rightarrow B$ be a chart at $w=\pi_{1}\left(w_{1}\right)$ and $\tilde{\sigma}_{i} \rightarrow R_{w}$ for $i=1,2$ the affine embeddings providing the local identification $\Phi_{e_{2} e_{1}}(s)$ whose graph is $\mathfrak{R}_{e_{2} e_{1}}$. Let $w_{2} \in \tilde{\sigma}_{2}$ be the vertex mapping to $0 \in R_{w}$. In verifying transitivity we may now restrict to a component $\mathfrak{R}_{f_{3} f_{2}}$ of $\mathfrak{R}$ that is non-trivial on $V\left(\sigma_{2}\right) \cap X_{w_{2}}$. For these there is another affine embedding $\tilde{\sigma}_{3} \rightarrow R_{w}$ that together with the already given embedding of $\tilde{\sigma}_{2}$ provides the local isomorphism $\Phi_{f_{3} f_{2}}(s)$ with graph $\Re_{f_{3} f_{2}}$. Let $w_{3} \in \tilde{\sigma}_{3}$ be the vertex mapping to $0 \in R_{w}$. Define $\tau$ to be the image of $\tilde{\sigma}_{1} \cap \tilde{\sigma}_{3} \subset R_{w}$ in $B$, and let $g_{i} \in \operatorname{Hom}\left(\tau, \sigma_{i}\right), i=1,3$ be the edges of the barycentric decomposition belonging to the faces $\tilde{\sigma}_{1} \cap \tilde{\sigma}_{3} \subset \tilde{\sigma}_{i}$. The pair $\left(g_{3}, g_{1}\right)$ parametrizes a unique component $\mathfrak{R}_{g_{3} g_{1}}$ of $\mathfrak{R}$, which is the graph of $\Phi_{g_{3} g_{1}}(s)$.

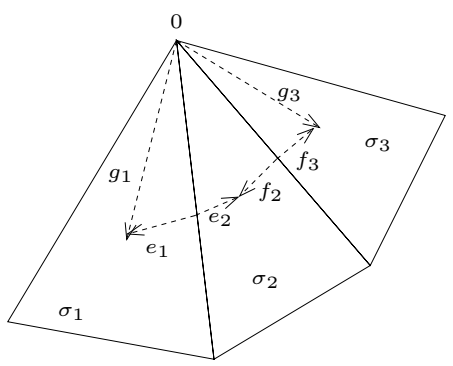

It is clear from toric geometry that the composition of $\Phi_{f_{3} f_{2}} \mid X_{w_{2}}$ with $\left.\Phi_{e_{2} e_{1}}\right|_{X_{w_{1}}}$ equals $\left.\Phi_{g_{3} g_{1}}\right|_{X_{w_{3}}}$ on the common domain of definition. As for the twisting by $s$, we observe that the cocycle condition implies that when restricted to $w_{i}$,

$$
s_{e_{1}}=s_{0 \tilde{\sigma}_{1}} s_{0 \omega}^{-1}, \quad s_{e_{2}}=s_{0 \tilde{\sigma}_{2}} s_{0 \omega}^{-1},
$$

where $\omega=\tilde{\sigma}_{1} \cap \tilde{\sigma}_{2}$ and with indices referring to the barycentric subdivision of $R_{w}$. In the definition of $\Phi_{e_{2} e_{1}}(s)$ on $X_{w_{1}}$ the term $s_{0 \omega}^{-1}$ therefore 
cancels:

$$
\left.\Phi_{e_{2} e_{1}}(s)\right|_{X_{w_{1}}}=s_{0 \tilde{\sigma}_{2}}^{-1} \circ \Phi_{e_{2} e_{1}} \circ s_{0 \tilde{\sigma}_{1}} .
$$

Similar formulae hold for $\Phi_{f_{3} f_{2}}$ and $\Phi_{g_{3} g_{1}}$. Hence

$$
\left.\Phi_{f_{3} f_{2}}(s) \circ \Phi_{e_{2} e_{1}}(s)\right|_{X_{w_{1}}}=\left.s_{0 \tilde{\sigma}_{3}}^{-1} \circ \Phi_{g_{3} g_{1}} \circ s_{0 \tilde{\sigma}_{1}}\right|_{X_{w_{1}}}=\left.\Phi_{g_{3} g_{1}}(s)\right|_{X_{w_{1}}} .
$$

This establishes transitivity.

q.e.d.

Definition 2.28. If $s$ are open gluing data for $\mathscr{P}$ over $S$, we set

$$
X_{0}(B, \mathscr{P}, s):=\left(\coprod_{\sigma \in \mathscr{P}_{\max }} V(\sigma) \times S\right) / \mathfrak{R} .
$$

By Lemma 2.27 this is an algebraic space locally of finite type over $S$.

It remains to show that this space is a direct limit for the functor $F_{S, s}$. We denote by

$$
p: \coprod_{\sigma \in \mathscr{P}_{\max }} V(\sigma) \times S \longrightarrow X_{0}(B, \mathscr{P}, s)
$$

the quotient morphism, and $p_{\sigma}: V(\sigma) \times S \rightarrow X_{0}(B, \mathscr{P}, s)$ the restriction of $p$ to $V(\sigma) \times S$.

Lemma 2.29. Let $s$ be open gluing data for $\mathscr{P}$ over $S$, and $\bar{s}$ the associated closed gluing data. There exists a system of maps $q_{\tau}: X_{\tau} \times$ $S \rightarrow X_{0}(B, \mathscr{P}, s)$ that is compatible with the functor $F_{S, \bar{s}}$; that is, for every $\tau_{1}, \tau_{2} \in \mathscr{P}$ and $e \in \operatorname{Hom}\left(\tau_{1}, \tau_{2}\right)$ we have

$$
q_{\tau_{2}}=q_{\tau_{1}} \circ F_{S, \bar{s}}(e) .
$$

Proof. We first define $q_{w}$ for $w \in \mathscr{P}$ a vertex. The set of maximal cones of the fan $\Sigma_{w}$ defining $X_{w}$ is in one-to-one correspondence with $\coprod_{\sigma \in \mathscr{P}_{\max }} \operatorname{Hom}(\{w\}, \sigma)$, so $X_{w}$ has an open covering indexed by this set. The affine open set $V_{e} \subset X_{w}$ corresponding to $e \in \operatorname{Hom}(\{w\}, \sigma)$ is what we denoted by $V(\sigma) \cap X_{\tilde{w}}$ in the proof of Lemma 2.27 for the unique lift $\tilde{w} \in \tilde{\sigma}$ distinguished by $e$. This gives a closed embedding

$$
\iota_{e}: V_{e} \longrightarrow V(\sigma)
$$

identifying $V_{e}$ with the irreducible component of $V(\sigma)$ corresponding to $\tilde{w}$. On $V_{e}$ define $q_{w}$ as composition

$$
q_{w}: V_{e} \times S \stackrel{\iota_{e} \times \operatorname{id}_{S}}{\longrightarrow} V(\sigma) \times S \stackrel{s_{e}^{-1}}{\longrightarrow} V(\sigma) \times S \stackrel{p}{\longrightarrow} X_{0}(B, \mathscr{P}, s) .
$$

(Technically, $s_{e}^{-1}$ only acts on the image of $\iota_{e} \times \mathrm{id}_{S}$, but it can be extended in any way we like to $V(\sigma) \times S$ for convenience of notation in this proof.) To check well-definedness let $\sigma_{1}, \sigma_{2} \in \mathscr{P}_{\max }$ and $e_{i} \in$ $\operatorname{Hom}\left(\{w\}, \sigma_{i}\right)$. Let $\tilde{\sigma}_{i} \subset R_{w}$ be the embeddings of the coverings of $\sigma_{i}$ into the chart at $w$ corresponding to $e_{i}$ as in the definition of $\mathfrak{R}$. Let $\tilde{\tau}_{i}=\tilde{\sigma}_{1} \cap \tilde{\sigma}_{2}$, viewed as subcell of $\tilde{\sigma}_{i}, \tau \in \mathscr{P}$ the image of $\tilde{\tau}_{i}, f_{i}: \tau \rightarrow \sigma_{i}$, 
$g: w \rightarrow \tau$ the induced morphisms, with $f_{i} \circ g=e_{i}$. By the definition of $\Re$

$$
\left.p\right|_{V\left(\tilde{\tau}_{2}\right) \times S}=\left.p\right|_{V\left(\tilde{\tau}_{1}\right) \times S} \circ \Phi_{f_{1} f_{2}}(s) .
$$

On the other hand, $\iota_{e_{i}}$ identifies $V_{e_{1}} \cap V_{e_{2}}$ with an irreducible component of $V\left(\tilde{\tau}_{i}\right) \subset V\left(\sigma_{i}\right)$, and these two embeddings are related by $\Phi_{f_{1} f_{2}}$ :

$$
\left.\iota_{e_{1}}\right|_{V_{e_{1}} \cap V_{e_{2}}}=\left.\Phi_{f_{1} f_{2}} \circ \iota_{e_{2}}\right|_{V_{e_{1}} \cap V_{e_{2}}} .
$$

On $V_{e_{1}} \cap V_{e_{2}}$ it follows

$$
\begin{aligned}
\left(\left.p\right|_{V\left(\tilde{\tau}_{1}\right) \times S}\right) \circ s_{e_{1}}^{-1} \circ\left(\iota_{e_{1}} \times \mathrm{id}_{S}\right) \\
=\left(\left.p\right|_{V\left(\tilde{\tau}_{1}\right) \times S}\right) \circ s_{e_{1}}^{-1} \circ\left(\Phi_{f_{1} f_{2}} \times \mathrm{id}_{S}\right) \circ\left(\iota_{e_{2}} \times \mathrm{id}_{S}\right) \\
=\left(\left.p\right|_{V\left(\tilde{\tau}_{1}\right) \times S}\right) \circ s_{e_{1}}^{-1} s_{f_{1}} \circ \Phi_{f_{1} f_{2}}(s) \circ s_{f_{2}}^{-1} \circ\left(\iota_{e_{2}} \times \mathrm{id}_{S}\right) \\
=\left(\left.p\right|_{V\left(\tilde{\tau}_{1}\right) \times S}\right) \circ s_{g}^{-1} \circ \Phi_{f_{1} f_{2}}(s) \circ s_{f_{2}}^{-1} \circ\left(\iota_{e_{2}} \times \mathrm{id}_{S}\right) \\
=\left(\left.p\right|_{V\left(\tilde{\tau}_{1}\right) \times S}\right) \circ \Phi_{f_{1} f_{2}}(s) \circ s_{g}^{-1} s_{f_{2}}^{-1} \circ\left(\iota_{e_{2}} \times \mathrm{id}_{S}\right) \\
=\left(\left.p\right|_{V\left(\tilde{\tau}_{1}\right) \times S}\right) \circ \Phi_{f_{1} f_{2}}(s) \circ s_{e_{2}}^{-1} \circ\left(\iota_{e_{2}} \times \mathrm{id}_{S}\right) \\
=\left(\left.p\right|_{V\left(\tilde{\tau}_{2}\right) \times S}\right) \circ s_{e_{2}}^{-1} \circ\left(\iota_{e_{2}} \times \mathrm{id}_{S}\right) .
\end{aligned}
$$

This shows well-definedness.

For non-minimal $\tau \in \mathscr{P}$ choose a vertex $w \in \tau$ and $g \in \operatorname{Hom}(\{w\}, \tau)$. Then put

$$
q_{\tau}=q_{w} \circ F_{S, \bar{s}}(g) .
$$

Since $F_{S, \bar{s}}$ is a functor, compatibility of these definitions follows once we convince ourselves of independence of the choices of $w$ and $g$. Let $w_{i} \in \tau$ be vertices and $g_{i} \in \operatorname{Hom}\left(\left\{w_{i}\right\}, \tau\right)$. Write $q_{\tau}^{i}$ for $q_{\tau}$ defined with $g_{i}$. Let $\sigma \in \mathscr{P}_{\max }$ and $f \in \operatorname{Hom}(\tau, \sigma)$. Putting $e_{i}=f \circ g_{i}$ we obtain the following commutative diagram.

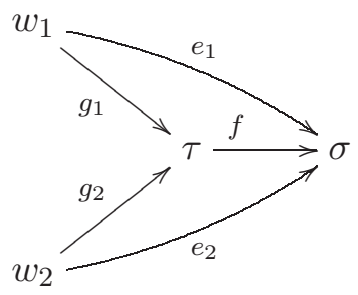

Each $e_{i}$ gives an identification of an open $V_{e_{i}} \subset X_{w_{i}}$ with an irreducible component of $V(\sigma)$. Then $F\left(g_{i}\right)^{-1}\left(V_{e_{i}}\right)$ is the open set in $X_{\tau}$ given by the maximal cone $K_{f} \in \Sigma_{\tau}$ corresponding to $f: \tau \rightarrow \sigma$, hence does not depend on $i$. This open set is $V_{f}$. Since the $V_{f}$ with $f \in$ $\coprod_{\sigma \in \mathscr{P}_{\max }} \operatorname{Hom}(\tau, \sigma)$ cover $X_{\tau}$, it is enough to show that the $q_{\tau}^{i}$ agree on $V_{f} \times S$.

Let $K_{e_{i}}, K_{g_{i}} \in \Sigma_{w_{i}}$ be the cones corresponding to $e_{i}$ and $g_{i}$. Recall from Definition 2.11 that $F_{S, s}\left(g_{i}\right)=\left(F\left(g_{i}\right) \times \mathrm{id}_{S}\right) \circ \bar{s}_{g_{i}}$ where $\left.F\left(g_{i}\right)\right|_{V_{f}}$ 
is induced by the description of cones

$$
K_{f}=\left(K_{e_{i}}+\mathbb{R} K_{g_{i}}\right) / \mathbb{R} K_{g_{i}} .
$$

On the other hand, $q_{w_{i}}$ involved the closed embedding $\iota_{e_{i}}: V_{e_{i}} \rightarrow V(\sigma)$ defined by identifying $K_{e_{i}}^{\vee}$ with a maximal face of $C(\sigma)^{\vee}$. Therefore, the composition

$$
V_{f} \stackrel{F\left(g_{i}\right)}{\longrightarrow} V_{e_{i}} \stackrel{\iota_{e_{i}}}{\longrightarrow} V(\sigma)
$$

is independent of $i$. Together with the cocycle condition we see that also

$$
s_{e_{i}}^{-1} \circ\left(\iota_{e_{i}} \times \operatorname{id}_{S}\right) \circ\left(F\left(g_{i}\right) \times \operatorname{id}_{S}\right) \circ \bar{s}_{g_{i}}=s_{f}^{-1} \circ\left(\left(\iota_{e_{i}} \circ F\left(g_{i}\right)\right) \times \operatorname{id}_{S}\right)
$$

does not depend on $i$. The composition with $p: V(\sigma) \times S \rightarrow X_{0}(B, \mathscr{P}, s)$ equals $\left.q_{\tau}^{i}\right|_{V_{f}}$, which is hence independent of $i$ too. $\quad$ q.e.d.

Proposition 2.30. $\coprod_{\tau} q_{\tau}: \coprod_{\tau} X_{\sigma} \times S \longrightarrow X_{0}(B, \mathscr{P}, s)$ is a direct limit for the functor $F_{S, \bar{s}}$.

Proof. We have to verify the universal property. Let $\psi_{\tau}: X_{\tau} \times S \rightarrow Z$ be a system of morphisms to an algebraic space that is compatible with $F_{S, \bar{s}}$. First we observe that $V(\sigma) \times S, \sigma \in \mathscr{P}_{\max }$, is a similar direct limit, but over the spaces $V_{f} \times S$ with $f \in \coprod_{\tau} \operatorname{Hom}(\tau, \sigma)$ and morphisms $\iota_{g}:=\left.F(g)\right|_{V_{f_{2}}}: V_{f_{2}} \rightarrow V_{f_{1}}$ for $f_{i}: \tau_{i} \rightarrow \sigma, g: \tau_{1} \rightarrow \tau_{2}, f_{1}=f_{2} \circ g$. Each $V_{f}$ is canonically an open subset of $X_{\tau}$. Consider the system of morphisms $\chi_{f}=\left.\psi_{\tau} \circ \bar{s}_{f}\right|_{V_{f} \times S}: V_{f} \times S \rightarrow Z, f \in \coprod_{\tau} \operatorname{Hom}(\tau, \sigma)$. This is compatible with the system $\left(V_{f} \times S, \iota_{g}\right)$. In fact, for $f_{i}, g$ as above,

$$
\left.F_{S, \bar{s}}(g)\right|_{V_{f_{2}} \times S}=\left.\left(F(g) \times \operatorname{id}_{S}\right)\right|_{V_{f_{2}} \times S} \circ \bar{s}_{g}=\bar{s}_{f_{1}} \circ\left(\iota_{g} \times \operatorname{id}_{S}\right) \circ \bar{s}_{f_{2}}^{-1} .
$$

Thus

$$
\chi_{f_{2}}=\left.\psi_{\tau_{2}} \circ \bar{s}_{f_{2}}\right|_{V_{f_{2}} \times S}=\left.\psi_{\tau_{1}} \circ F_{S, \bar{s}}(g) \circ \bar{s}_{f_{2}}\right|_{V_{f_{2}} \times S}=\chi_{f_{1}} \circ\left(\iota_{g} \times \mathrm{id}_{S}\right) .
$$

Hence we obtain a morphism

$$
\chi(\sigma): V(\sigma) \times S \longrightarrow Z .
$$

To descend to $X_{0}(B, \mathscr{P}, s)$ it remains to show that the $\chi(\sigma)$ commute with the local isomorphisms $\Phi_{e_{1} e_{2}}(s)$ whose graphs define $\mathfrak{R}$ locally. As in the proof of transitivity of $\mathfrak{R}$ we may restrict to one irreducible component at a time. If $e_{i}: \tau \rightarrow \sigma_{i}$ this amounts to choosing a vertex $w \in \tau$ and homomorphisms $f_{i}:\{w\} \rightarrow \sigma_{i}$ with $f_{i}=e_{i} \circ g$ for some $g:\{w\} \rightarrow \tau$. A chart at $w$ reduces to the case $\sigma_{i} \subset M \otimes_{\mathbb{Z}} \mathbb{R}, w=0$ and $\tau=\sigma_{1} \cap \sigma_{2}$. This situation gave open embeddings $V(\tau) \subset V\left(\sigma_{i}\right)$, and restricted to the irreducible component $V_{g} \subset V(\tau)$ labelled by $w=0 \in \tau$ 
they defined $\Phi_{e_{1} e_{2}}$. We obtain the following diagram.

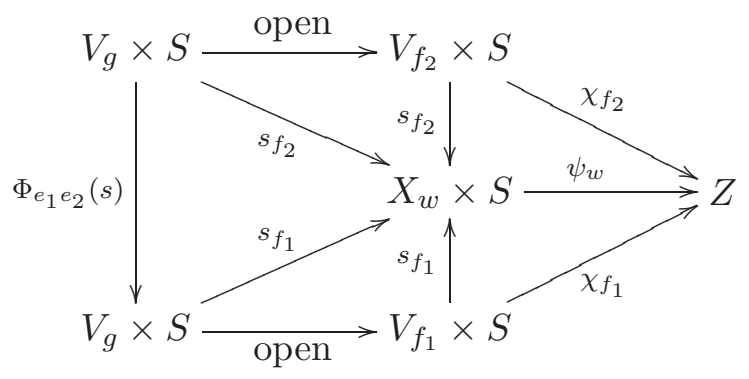

The arrows labelled "open" are open embeddings canonically defined by toric geometry, while the labels $s_{f_{i}}$ denote similar open embeddings but twisted by the action of $s_{f_{i}}$ on $X_{w}$. In the case without self-intersections, $V(\tau)=V\left(\sigma_{1}\right) \cap V\left(\sigma_{2}\right), V_{g}=V(\tau) \cap X_{w}$ and $V_{f_{i}}=V(\sigma) \cap X_{w}$. In general $V_{g}$ and $V_{f_{i}}$ are open subschemes of $X_{w}$. Therefore the upper and lower triangles commute trivially. The two triangles with common side $\psi_{w}$ commute by the definition of $\chi_{f_{i}}$, and the triangle on the left commutes by the definition of $\Phi_{e_{1} e_{2}}(s)$. Therefore the whole diagram commutes, and following the two paths from $V_{g} \times S$ to $Z$ via $X_{w} \times S$ shows the desired compatibility of $\chi_{f}$ with our equivalence relation. q.e.d.

Proposition 2.31. $X_{0}(B, \mathscr{P}, s)$ is a separated algebraic space. If $B$ is compact and $S$ is a separated scheme, then $X_{0}(B, \mathscr{P}, s)$ is a proper algebraic space over $S$.

Proof. An algebraic space is separated if the equivalence relation defining it is a closed embedding ([30], Def. II.1.6) and this is the case by Lemma 2.27. Thus in addition if $S$ is separated, so is the morphism $f: X_{0}(B, \mathscr{P}, s) \rightarrow S$ ([30], Prop. II.3.10). If $B$ is compact, $X_{0}(B, \mathscr{P}, s)$ is clearly of finite type over $S$ as $\mathscr{P}$ must be a finite set, so that $X_{0}(B, \mathscr{P}, s)$ has an étale cover of finite type over $S$. Finally, to show $f$ is proper, note that

$$
q: \coprod_{v \in \mathscr{P}} X_{v} \times S \longrightarrow X_{0}(B, \mathscr{P}, s)
$$

given by $q_{v}: X_{v} \times S \rightarrow X_{0}(B, \mathscr{P}, s)$ on $X_{v} \times S$ is a surjective map, and $\coprod X_{v} \times S \rightarrow S$ is a proper map. Then $f$ is also proper (see [22], II 5.4.3 (ii) in the scheme case, but the same proof works for algebraic spaces).

q.e.d.

Next, we consider when two sets of open gluing data give isomorphic spaces. Suppose $s, s^{\prime}$ are open gluing data for $(B, \mathscr{P})$ over $S$. We are interested in understanding when there are isomorphisms

$$
\varphi: X_{0}(B, \mathscr{P}, s) \longrightarrow X_{0}\left(B, \mathscr{P}, s^{\prime}\right) .
$$


We will only be interested in isomorphisms $\varphi$ with $\varphi\left(q_{\tau}\left(X_{\tau} \times S\right)\right)=$ $q_{\tau}^{\prime}\left(X_{\tau} \times S\right)$; any other isomorphism will reflect some global symmetry of $B$. In this case, we obtain a commutative diagram for each $\tau \in \mathscr{P}$

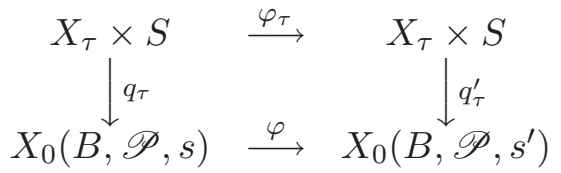

If $\varphi_{\tau}$ also preserves toric strata of $X_{\tau} \times S$, which implies it is induced by an element $\bar{t}_{\tau} \in \mathcal{Q}_{\tau} \otimes \mathbb{G}_{m}(S)$, then we say the isomorphism $\varphi$ preserves $B$. Clearly the component of the identity of the automorphism group of $X_{0}(B, \mathscr{P}, s)$ is the set of automorphisms preserving $B$.

A simple example of an automorphism not preserving $B$ is when $B=\mathbb{R} / \mathbb{Z}$ and $X_{0}(B, \mathscr{P}, s)$ is isomorphic to a nodal cubic, say $y^{2} z=$ $x^{3}+x^{2} z$ in $\mathbb{P}^{2}$. Then the automorphism $y \mapsto-y$ induces a map on the normalization interchanging toric strata.

\section{Proposition 2.32.}

1) Let $s, s^{\prime}$ be two open gluing data for $(B, \mathscr{P})$ over $S$. Then there exists an isomorphism

$$
\varphi: X_{0}(B, \mathscr{P}, s) \stackrel{\cong}{\longrightarrow} X_{0}\left(B, \mathscr{P}, s^{\prime}\right)
$$

preserving $B$ if and only if $s / s^{\prime} \in B^{1}\left(\mathscr{P}, \mathcal{Q}_{\mathscr{P}} \otimes \mathbb{G}_{m}(S)\right)$.

2) The map

$$
H^{1}\left(\mathscr{P}, \mathcal{Q} \mathscr{P} \otimes \mathbb{G}_{m}(S)\right) \longrightarrow H^{1}\left(\mathscr{W}, \mathcal{Q}_{\mathscr{P}} \otimes \mathbb{G}_{m}(S)\right)
$$

induced by taking open gluing data to closed gluing data is an inclusion.

Proof.

1) If $s / s^{\prime} \in B^{1}\left(\mathscr{P}, \mathcal{Q}_{\mathscr{P}} \otimes \mathbb{G}_{m}(S)\right)$, then there exists $t_{\sigma} \in \check{P} M(\sigma)$ for all $\sigma \in \mathscr{P}$ such that for $e: \tau \rightarrow \sigma, s_{e}^{\prime}=\left.t_{\sigma}^{-1}\right|_{\tau} s_{e} t_{\tau}$. Then the isomorphism

$$
\coprod_{\sigma \in \mathscr{P}_{\max }} V(\sigma) \times S \longrightarrow \coprod_{\sigma \in \mathscr{P}_{\max }} V(\sigma) \times S
$$

which is given by $t_{\sigma}$ on $V(\sigma) \times S$ is compatible with the equivalence relation defined by $s$ on the domain and the equivalence relation defined by $s^{\prime}$ on the range. Thus this isomorphism descends to give an isomorphism

$$
X(B, \mathscr{P}, s) \longrightarrow X\left(B, \mathscr{P}, s^{\prime}\right) .
$$

Conversely, if there is an isomorphism $\varphi$ preserving $B$, then for each $\sigma \in \mathscr{P}_{\max }, e: \tau \rightarrow \sigma$, we have $V_{e} \subseteq X_{\tau}$ the open subset of $X_{\tau}$ given by Definition 2.22. The isomorphism $\varphi_{\tau}: X_{\tau} \times S \rightarrow X_{\tau} \times S$ induces an isomorphism $\varphi_{e}: V_{e} \times S \rightarrow V_{e} \times S$, and these patch to give an 
isomorphism $\tilde{\varphi}_{\sigma}: V(\sigma) \times S \rightarrow V(\sigma) \times S$, which is induced by some $t_{\sigma} \in P M(\sigma)$. Then $\varphi$ is induced by

$$
\tilde{\varphi}=\coprod \tilde{\varphi}_{\sigma}: \coprod_{\sigma \in \mathscr{P}_{\max }} V(\sigma) \times S \longrightarrow \coprod_{\sigma \in \mathscr{P}_{\max }} V(\sigma) \times S .
$$

Now set $s_{e}^{\prime \prime}=s_{e} t_{\sigma}^{-1}$ whenever $e: \tau \rightarrow \sigma \in \mathscr{P}_{\max }, s_{e}^{\prime \prime}=s_{e}$ otherwise. Then $s^{\prime \prime}$ is congruent to $s \bmod B^{1}\left(\mathscr{P}, \mathcal{Q}_{\mathscr{P}} \otimes \mathbb{G}_{m}(S)\right)$, so as above we obtain an isomorphism

$$
\psi: X_{0}\left(B, \mathscr{P}, s^{\prime \prime}\right) \longrightarrow X_{0}(B, \mathscr{P}, s)
$$

induced by $t_{\sigma}^{-1}$ on $V(\sigma)$. Furthermore, the composition $\varphi \circ \psi$ is then induced by the identity maps on $V(\sigma) \times S$ for all $\sigma \in \mathscr{P}_{\max }$. Thus, by replacing $s$ by $s^{\prime \prime}$, we can assume $\tilde{\varphi}_{\sigma}$ is the identity for each $\sigma \in \mathscr{P}_{\max }$, and thus the equivalence relations $\mathfrak{R}$ and $\mathfrak{R}^{\prime}$ defined by $s$ and $s^{\prime}$ must be the same.

Thus in particular, whenever $\left(e_{1}, e_{2}\right)$ is a maximal pair,

$$
\left(s_{e_{1}}^{\prime}\right)^{-1} \circ \Phi_{e_{1} e_{2}} \circ s_{e_{2}}^{\prime}=s_{e_{1}}^{-1} \circ \Phi_{e_{1} e_{2}} \circ s_{e_{2}} .
$$

In particular,

$$
s_{e_{2}} / s_{e_{2}}^{\prime}=s_{e_{1}} / s_{e_{1}}^{\prime} \text {. }
$$

Now for each $\tau \in \mathscr{P} \backslash \mathscr{P}_{\max }$, choose some morphism $e: \tau \rightarrow \sigma \in$ $\mathscr{P}_{\max }$, and define $t_{\tau}=s_{e} / s_{e}^{\prime}$. We first need to check that this is independent of $e$. Given a diagram

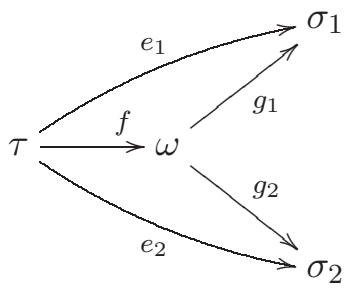

with $\left(g_{1}, g_{2}\right)$ a maximal pair, then using $(2.1)$ for $\left(g_{1}, g_{2}\right)$, we have

$$
\frac{s_{e_{1}}}{s_{e_{1}}^{\prime}}=\frac{\left.s_{g_{1}}\right|_{\tau} s_{f}}{\left.s_{g_{1}}^{\prime}\right|_{\tau} s_{f}^{\prime}}=\frac{\left.s_{g_{2}}\right|_{\tau} s_{f}}{\left.s_{g_{2}}^{\prime}\right|_{\tau} s_{f}^{\prime}}=\frac{s_{e_{2}}}{s_{e_{2}}^{\prime}} .
$$

Finally, with $t_{\sigma}=1$ for $\sigma \in \mathscr{P}_{\max }$, we note that for any $e: \tau_{1} \rightarrow \tau_{2}$,

$$
\left.t_{\tau_{1}}^{-1} s_{e} t_{\tau_{2}}\right|_{\tau_{1}}=s_{e}^{\prime} .
$$

Indeed, given a map $f: \tau_{2} \rightarrow \sigma \in \mathscr{P}_{\max }, g=f \circ e$,

$$
\left.t_{\tau_{1}}^{-1} s_{e} t_{\tau_{2}}\right|_{\tau_{1}}=\left.\left.t_{\tau_{1}}^{-1} s_{g} s_{f}^{-1}\right|_{\tau_{1}} t_{\tau_{2}}\right|_{\tau_{1}}=\left.\frac{s_{g}^{\prime}}{s_{g}} s_{g} s_{f}^{-1}\right|_{\tau_{1}} \frac{\left.s_{f}\right|_{\tau_{1}}}{\left.s_{f}^{\prime}\right|_{\tau_{1}}}=\left.s_{g}^{\prime}\left(s_{f}^{\prime}\right)^{-1}\right|_{\tau_{1}}=s_{e}^{\prime} .
$$

Thus $s$ and $s^{\prime}$ are equivalent open gluing data. 
2) Clearly if $s \in B^{1}\left(\mathscr{P}, \mathcal{Q}_{\mathscr{P}} \otimes \mathbb{G}_{m}(S)\right)$ is induced by $t=\left(t_{\tau}\right)_{\tau \in \mathscr{P}}$,

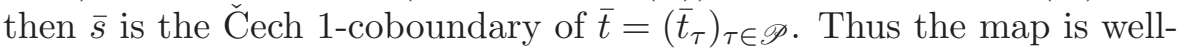
defined. In addition, if $s \in Z^{1}\left(\mathscr{P}, \mathcal{Q}_{\mathscr{P}} \otimes \mathbb{G}_{m}(S)\right)$ is mapped to a Čech 1-coboundary, say the coboundary of $\left(\bar{t}_{\tau}\right)_{\tau \in \mathscr{P}}$ with $\bar{t}_{\tau} \in \Gamma\left(W_{\tau}, \mathcal{Q}_{\mathscr{P}} \otimes\right.$ $\left.\mathbb{G}_{m}(S)\right)=\mathcal{Q}_{\tau} \otimes \mathbb{G}_{m}(S)$, then $\bar{t}_{\tau}$ induces a map $\varphi_{\tau}: X_{\tau} \rightarrow X_{\tau}$ and then an isomorphism of $X_{0}(B, \mathscr{P}, s)=\lim F_{S, \bar{s}}$ with $X_{0}(B, \mathscr{P}, 1)=$ $\lim F_{S, 1}$, where 1 denotes trivial open gluing data. But then by (1), $s \in B^{1}\left(\mathscr{P}, \mathcal{Q}_{\mathscr{P}} \otimes \mathbb{G}_{m}(S)\right)$. Thus the map is injective.

q.e.d.

Remark 2.33. Thus we see that $H^{1}\left(\mathscr{W}, \mathcal{Q} \mathscr{P} \otimes \mathbb{G}_{m}(S)\right)$ parametrizes a set of gluings of the components $X_{\tau}$, while $H^{1}\left(\mathscr{P}, \mathcal{Q}_{\mathscr{P}} \otimes \mathbb{G}_{m}(S)\right)$ is a subgroup parametrizing gluings which are "locally trivial," i.e., étale locally isomorphic to things of the form $V(\sigma)$. It is only these locally trivial gluings which will play a role in the theory. The difference between open and closed gluing data can be exhibited locally. Given an integral lattice polytope $\sigma \subseteq M_{\mathbb{R}}, \mathcal{Q}_{\mathscr{P}}$ still makes sense as a sheaf just on $\sigma$, as does the open cover $\overline{\mathscr{W}}$, so $H^{1}\left(\mathscr{W}, \mathcal{Q} \mathscr{P} \otimes \mathbb{G}_{m}(S)\right)$ still makes sense, and gives regluings of irreducible components of $V(\sigma)$. If $H^{1}(\mathscr{W}, \mathcal{Q} \mathscr{P} \otimes$ $\left.\mathbb{G}_{m}(S)\right) \neq 0$, there are regluings of the irreducible components of $V(\sigma)$ which yield spaces not isomorphic to $V(\sigma)$. One example of such a $\sigma$ is an octahedron in $\mathbb{R}^{3}$; one can show $H^{1}\left(\mathscr{W}, \mathcal{Q}_{\mathscr{P}} \otimes \mathbb{G}_{m}(S)\right) \neq 0$ by a dimension counting argument. Somewhat mysteriously, this group seems to have something to do with the Brauer group of the toric variety $X\left(\check{\Sigma}_{\sigma}\right)$ : see $[\mathbf{1 1}]$.

We also note that one can easily show (using similar methods as in Lemma 5.5) that $\mathscr{W}$ is an acyclic covering for $\mathcal{Q}_{\mathscr{P}} \otimes \mathbb{G}_{m}(S)$, and thus $H^{1}\left(\mathscr{W}, \mathcal{Q}_{\mathscr{P}} \otimes \mathbb{G}_{m}(S)\right) \cong H^{1}\left(B, \mathcal{Q}_{\mathscr{P}} \otimes \mathbb{G}_{m}(S)\right)$.

Next, we make the connection between the cone and fan pictures. Given open gluing data $s, X_{0}(B, \mathscr{P}, s)$ is not necessarily projective even if we have specified ample line bundles on each irreducible component compatible with identifications. It turns out giving such data is equivalent to giving a strictly convex piecewise linear function $(B, \mathscr{P})$. However, there is still an obstruction to gluing these line bundles to obtain an ample line bundle on $X_{0}(B, \mathscr{P}, s)$. This obstruction, depending on $s$, is the map described in the following theorem. If this obstruction vanishes, $X_{0}(B, \mathscr{P}, s)$ will be projective and arise from the cone picture of a discrete Legendre transform of $(B, \mathscr{P})$.

Theorem 2.34. Let $B$ be an integral affine manifold with singularities with a toric polyhedral decomposition $\mathscr{P}$ and a strictly convex integral piecewise linear multi-valued function $\varphi$. Let $S=\operatorname{Spec} A$ be an affine scheme. Let $(\check{B}, \check{\mathscr{P}}, \check{\varphi})$ be the discrete Legendre transform of $(B, \mathscr{P}, \varphi)$. Then there is a group homomorphism

$$
o: H^{1}\left(\mathscr{P}, \mathcal{Q} \mathscr{P} \otimes \mathbb{G}_{m}(S)\right) \longrightarrow H^{2}\left(B, \mathbb{G}_{m}(S)\right)
$$


such that if $o(s)=1$, there is a choice of gluing data $\check{s}$ for the cone picture for $\check{\mathscr{P}}$ over $S$ with an isomorphism

$$
\check{X}_{0}(\check{B}, \check{\mathscr{P}}, \check{s}) \cong X_{0}(B, \mathscr{P}, s) .
$$

In particular, $X_{0}(B, \mathscr{P}, s)$ is a projective scheme.

Proof. If $\sigma \in \mathscr{P}$ is a cell, then $\varphi$ induces a strictly convex piecewise linear function $\varphi_{\sigma}$ on $\Sigma_{\sigma}$, well-defined up to linear functions, and hence an ample line bundle $\mathcal{L}_{\sigma}$ on $X_{\sigma}$. Then it is a standard fact of toric geometry that if $\widetilde{\sigma} \subseteq \mathcal{Q}_{\sigma, \mathbb{R}}^{*}$ is the corresponding Newton polytope of $\mathcal{L}_{\sigma}$ (as defined in the definition of discrete Legendre transform in $\S 1.4$ ), $C(\widetilde{\widetilde{\sigma}}) \subseteq \mathcal{Q}_{\sigma, \mathbb{R}}^{*} \oplus \mathbb{R}, \check{P}_{\breve{\sigma}}=C(\widetilde{\widetilde{\sigma}}) \cap\left(\mathcal{Q}_{\sigma}^{*} \oplus \mathbb{Z}\right), R_{\check{\sigma}}=A\left[\check{P}_{\check{\sigma}}\right]$, then

$$
\operatorname{Proj} R_{\breve{\sigma}} \cong X_{\sigma},
$$

with

$$
\mathcal{O}_{\operatorname{Proj} R_{\check{\sigma}}}(1) \cong \mathcal{L}_{\sigma} .
$$

On the other hand, $R_{\breve{\sigma}}$ is the ring given by Definition 2.1 for $\check{\sigma} \in \check{\mathscr{P}}$, so $\check{X}_{\check{\sigma}} \cong X_{\sigma}$. Furthermore, it is easy to see that if $e: \tau \rightarrow \sigma$ (or equivalently $e: \check{\sigma} \rightarrow \check{\tau}$ ) then

$$
F(e): X_{\sigma} \longrightarrow X_{\tau}
$$

coincides with

$$
\operatorname{Proj} \check{F}(e): \check{X}_{\check{\sigma}} \longrightarrow \check{X}_{\check{\tau}} \text {. }
$$

Now consider the open gluing data for the fan picture $s \in Z^{1}\left(\mathscr{P}, \mathcal{Q}_{\mathscr{P}} \otimes\right.$ $\left.\mathbb{G}_{m}(S)\right)$, with associated closed gluing data $\bar{s}$, with $\bar{s}_{e} \in \Gamma\left(W_{e}, \mathcal{Q}_{\mathscr{P}} \otimes\right.$ $\left.\mathbb{G}_{m}(S)\right) \cong \mathcal{Q}_{\sigma} \otimes \mathbb{G}_{m}(S)$ for $e: \tau \rightarrow \sigma$. There is a natural isomorphism between $\mathcal{Q}_{\sigma}$ and $\left(\Lambda_{\check{\sigma}}^{\check{B}}\right)^{*}$. On the other hand, choose some embedding $\widetilde{\sigma} \subseteq$ $\Lambda_{\check{\sigma}, \mathbb{R}}^{\check{B}}$. Then we can identify $\operatorname{Hom}\left(\check{P}_{\check{\sigma}}, \mathbb{G}_{m}(S)\right)$ with $\left(\Lambda_{\check{\sigma}}^{\check{B}} \oplus \mathbb{Z}\right)^{*} \otimes \mathbb{G}_{m}(S)$. However, this is non-canonical as it depends on the embedding of $\widetilde{\sigma}$; translating $\widetilde{\sigma}$ will change this identification. We can however use the inclusion of $\left(\Lambda_{\check{\sigma}}^{\check{B}}\right)^{*} \otimes \mathbb{G}_{m}(S)$ in $\left(\Lambda_{\check{\sigma}}^{\check{B}} \oplus \mathbb{Z}\right)^{*} \otimes \mathbb{G}_{m}(S)$ to identify $\bar{s}_{e}$ with an element $\check{s}_{e}$ of $\operatorname{Hom}\left(\check{P}_{\check{\sigma}}, \mathbb{G}_{m}(S)\right)$. Now $\left(\check{s}_{e}\right)$ may not satisfy the 1-cocycle condition for gluing data in the cone picture because of the arbitrary nature of this identification. In general, because $\bar{s}$ satisfied the 1-cocycle condition, given $e_{1}: \check{\sigma}_{1} \rightarrow \check{\sigma}_{2}, e_{2}: \check{\sigma}_{2} \rightarrow \check{\sigma}_{3}, e_{3}=e_{2} \circ e_{1}$, we have

$$
\left.\check{s}_{e_{1}} \cdot \check{s}_{e_{2}}\right|_{\check{P}_{\check{\sigma}_{1}}} \cdot \check{s}_{e_{3}}^{-1}: \check{P}_{\check{\sigma}_{1}} \longrightarrow \mathbb{G}_{m}(S)
$$

factors through the well-defined projection $\check{P}_{\check{\sigma}_{1}} \rightarrow \mathbb{Z}$. Thus we can consider $\left(\left.\check{s}_{e_{1}} \cdot \check{s}_{e_{2}}\right|_{\check{P}_{\check{\sigma}_{1}}} \cdot \check{s}_{e_{3}}^{-1}\right)(1) \in \mathbb{G}_{m}(S)$. This assigns to each twodimensional simplex in $\operatorname{Bar}(\check{\mathscr{P}})$ an element of $\mathbb{G}_{m}(S)$, and it is easy to see from the construction that this defines a 2-cocycle for simplicial cohomology of $B$, i.e., an element of $H^{2}\left(B, \mathbb{G}_{m}(S)\right)$. This defines the map $o$, and it is easy to see $o(s)$ only depends on the class of $s$ in $H^{1}\left(\mathscr{P}, \mathcal{Q}_{\mathscr{P}} \otimes \mathbb{G}_{m}(S)\right)$. 
Now suppose $o(s)=1$. Then there exists a 1 -cochain $\left(h_{e}\right), h_{e} \in$ $\mathbb{G}_{m}(S)$, e running over all edges of $\operatorname{Bar}(\mathscr{P})$ with

$$
\left(\left.\check{s}_{e_{1}} \cdot \check{s}_{e_{2}}\right|_{\check{P}_{\check{\sigma}_{1}}} \cdot \check{s}_{e_{3}}^{-1}\right)(1)=h_{e_{1}} \cdot h_{e_{2}} \cdot h_{e_{3}}^{-1} .
$$

Interpreting $h_{e}$ for $e: \check{\tau} \rightarrow \check{\sigma}$ as a map $\check{P}_{\check{\tau}} \rightarrow \mathbb{G}_{m}(S)$ given as the composition of the projection $\check{P}_{\check{\tau}} \rightarrow \mathbb{Z}$ and the map $n \mapsto h_{e}^{n}$, we can define

$$
\check{s}_{e}^{\prime}=\check{s}_{e} \cdot h_{e}^{-1} .
$$

It is then clear that $\check{s}^{\prime}=\left(\check{s}_{e}^{\prime}\right)$ are gluing data in the cone picture, as the 1-cocycle condition is satisfied. Replacing $\check{s}$ by $\check{s}^{\prime}$, it is easy to see that for $e: \tau \rightarrow \sigma$, we obtain a commutative diagram

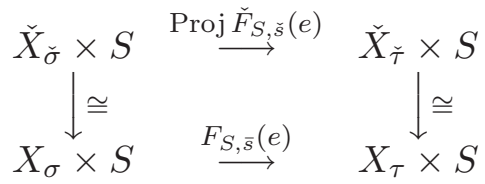

Taking limits yields an isomorphism

$$
X_{0}(B, \mathscr{P}, s) \cong \check{X}_{0}(\check{B}, \check{\mathscr{P}}, \check{s}) .
$$

q.e.d.

Thus we see that a strictly convex integral piecewise linear multivalued function on $B$ in particular gives rise to a choice of ample line bundle on $X_{0}(B, \mathscr{P}, s)$ for some $s$ (though not necessarily for all $s$ unless $\left.H^{2}\left(B, \mathbb{G}_{m}(S)\right)=0\right)$. However, there is also a choice involved. There might be different choices of $\left(h_{e}\right)$ in the proof above, giving different choices of gluing data in the fan picture. This choice is parametrized by $H^{1}\left(B, \mathbb{G}_{m}(S)\right)$. While this choice doesn't affect the underlying scheme, it does change the line bundle.

Example 2.35. If $B$ is as in Example 1.18, then $X_{0}(B, \mathscr{P}, 1)$ coincides with $\check{X}_{0}(\check{B}, \check{\mathscr{P}}, 1)$, where $\check{B}$ is obtained from the reflexive dual $\check{\Xi}$ of $\Xi$. (See Examples 1.53 and 2.6).

Example 2.36. According to Remark $2.19 X_{0}(B, \mathscr{P}, s)$ is a scheme if the cells of the polyhedral decomposition do not self-intersect. Even with self-intersections it might be a scheme, for example in dimension one. Here is an example where the property of being a scheme rather than an algebraic space depends on algebraic relations between the gluing parameters.

As in Examples 2.2 and 2.5 consider $B=\mathbb{R}^{2} / \mathbb{Z}^{2}$ with only one maximal cell coming from $\tilde{\sigma}=[0,1] \times[0,1] \subset \mathbb{R}^{2}$, but now viewed in the fan picture, with $S=\operatorname{Spec} k$. Then $X_{v}=\mathbb{P}^{1} \times \mathbb{P}^{1}$ for the unique vertex $v \in B$, and closed gluing data $s=\left(s_{e}\right)_{e: \tau \rightarrow \sigma}$ provide toric identifications $\{0\} \times \mathbb{P}^{1} \stackrel{\cong}{\rightrightarrows}\{\infty\} \times \mathbb{P}^{1}$ and $\mathbb{P}^{1} \times\{0\} \stackrel{\cong}{\rightrightarrows} \mathbb{P}^{1} \times\{\infty\}$ given by the actions 
of some $\alpha, \beta \in k^{\times}$respectively. Here $0, \infty \in \mathbb{P}^{1}$ denote the two zerodimensional torus orbits.

Now if $X=X_{0}(B, \mathscr{P}, s)$ is a scheme there exist effective divisors $D \subset X$ through the distinguished point $o \in X$ of multiplicity four and not containing a component of $X_{\text {sing }}$. Such $D$ can be obtained by taking the closure in $X$ of a Cartier divisor on an affine neighbourhood of $o$. A priori this leaves us with a Weil divisor. To obtain a Cartier divisor consider the $\mathbb{Z} / 2 \times \mathbb{Z} / 2$-action on $X$ defined on the normalization $\mathbb{P}^{1} \times \mathbb{P}^{1}$ by the toric automorphisms on each of the two factors exchanging the two zero-dimensional torus orbits. Summing over this action we may assume that $D$ is $\mathbb{Z} / 2 \times \mathbb{Z} / 2$-invariant. We claim that $D$ is now Cartier. In fact, this is only questionable at points $p \in\left|D \cap X_{\text {sing }}\right| \backslash\{o\}$. There is an étale neighbourhood of $p$ of the form Spec $k[x, y, z] /(x y)$ with one of the $\mathbb{Z} / 2$-factors exchanging the two irreducible components. (This could be made explicit by lifting $s$ to open gluing data, which is always possible in this case, and using the étale cover $V(\sigma) \rightarrow X$.) Let $D_{1}+D_{2}$ be the decomposition of $D$ according to the two irreducible components of this étale neighbourhood. Then since $D$ is $\mathbb{Z} / 2 \times \mathbb{Z} / 2$-invariant, $D_{1} \cap X_{\text {sing }}=$ $D_{2} \cap X_{\text {sing. }}$. Therefore there exist $f_{1}(x, z), f_{2}(y, z)$ defining $D_{1}, D_{2}$ on their components respectively, with $f_{1}(0, z)=f_{2}(0, z)$. Then $D$ is defined by $f_{1}(x, z)+f_{2}(y, z)-f_{1}(0, z)$. So $D$ is in fact Cartier.

The pull-back of $D$ to the normalization $\mathbb{P}^{1} \times \mathbb{P}^{1} \rightarrow X$ is now given by a bihomogeneous polynomial

$$
F=\sum_{\substack{\mu=0, \ldots, d \\ \nu=0, \ldots, e}} a_{\mu \nu} x^{\mu} y^{d-\mu} u^{\nu} v^{e-\nu}
$$

with $a_{00} \neq 0, d, e>0$, and such that

$$
F(x, y, 0,1)=c \cdot F(\alpha x, y, 1,0), \quad F(0,1, u, v)=c^{\prime} \cdot F(1,0, \beta u, v),
$$

for some $c, c^{\prime} \in k^{\times}$. Comparing coefficients gives the relations

$$
a_{\mu 0}=c \cdot \alpha^{\mu} a_{\mu e}, \quad a_{0 \nu}=c^{\prime} \cdot \beta^{\nu} a_{d \nu},
$$

for all $\mu, \nu$. This gives two ways to compute $a_{00}$ from $a_{d e}$ :

$$
\begin{aligned}
a_{00}=c a_{0 e} & =c c^{\prime} \beta^{e} a_{d e}, \\
a_{00}=c^{\prime} a_{d 0} & =c^{\prime} c \alpha^{d} a_{d e} .
\end{aligned}
$$

Thus a necessary condition for such a divisor $D$ to exist is an algebraic relation of the type

$$
\alpha^{d}=\beta^{e},
$$

with $d, e>0$, between the gluing parameters $\alpha$ and $\beta$.

Conversely, if such a relation exists the same considerations give sufficiently many Cartier divisors on $X$ separating points and tangents. Hence in this case $X$ is projective. In fact, such $X$ arise from the cone picture for a rectangle with sides of lengths $d$ and $e$. 
We compute some invariants of $X_{0}(B, \mathscr{P}, s)$.

Proposition 2.37. Let $B$ be an integral affine manifold with singularities and toric polyhedral decomposition $\mathscr{P}$. Let $s$ be open gluing data for $(B, \mathscr{P})$ over $S=\operatorname{Spec}(A)$, and $X=X_{0}(B, \mathscr{P}, s)$. Then

$$
H^{i}\left(X, \mathcal{O}_{X}\right) \cong H^{i}(B, A) \text {. }
$$

Proof. Let

$$
\mathscr{C}_{\mathrm{bct}}^{k}=\bigoplus_{\substack{\sigma_{0} \rightarrow \cdots \rightarrow \sigma_{k} \\ \sigma_{i} \neq \sigma_{i+1}}} q_{\sigma_{k} *} \mathcal{O}_{X_{\sigma_{k}} \times S},
$$

where for $\tau \in \mathscr{P}, q_{\tau}: X_{\tau} \times S \rightarrow X$ is the map constructed in Lemma 2.29. Define a differential $d_{\mathrm{bct}}$ as in $\S$ A.3. Then we show in Example A.3 that $\mathscr{C}_{\text {bct }}^{\bullet}$ is exact. There is also a natural inclusion $\mathcal{O}_{X} \rightarrow \mathscr{C}_{\text {bct }}^{0}$ given by pull-back of functions, and it is easy to check as in Example A.3 that this makes $\mathscr{C}_{\text {bct }}^{\bullet}$ into a resolution of $\mathcal{O}_{X}$. Now

$$
H^{i}\left(X_{\sigma_{k}} \times S, \mathcal{O}_{X_{\sigma_{k}} \times S}\right)= \begin{cases}A & i=0 \\ 0 & i>0\end{cases}
$$

as $X_{\sigma_{k}} \times S$ is proper over $S$, since $\Sigma_{\sigma_{k}}$ is a complete fan. It follows that $H^{i}\left(X, \mathcal{O}_{X}\right)=H^{i}\left(\Gamma\left(X, \mathscr{C}^{\bullet}\right)\right)$. However, $\Gamma\left(X, \mathscr{C}^{\bullet}\right)$ coincides with the complex of simplicial cochains with coefficients in the ring $A$ with respect to the barycentric triangulation $\operatorname{Bar}(\mathscr{P})$. Thus $H^{i}(\Gamma(X, \mathscr{C} \bullet))=$ $H^{i}(B, A)$ as desired.

q.e.d.

For later reference let us also record here an interpretation of $H^{1}(B$, $\mathbb{G}_{m}(S)$ ). We say a line bundle on a scheme $X$ over $S$ is numerically trivial if its pull-back to any complete regular curve defined over a geometric point of $S$ has degree zero. The set of isomorphism classes of numerically trivial line bundles of $X$ form a subgroup $\operatorname{Pic}^{\tau}(X) \subset \operatorname{Pic}(X)$.

Proposition 2.38. Let $B$ be an integral affine manifold with singularities and toric polyhedral decomposition $\mathscr{P}$. Let $s$ be open gluing data for $(B, \mathscr{P})$ over $S=\operatorname{Spec}(A)$, and $X=X_{0}(B, \mathscr{P}, s)$. Then

$$
\operatorname{Pic}^{\tau}(X)=H^{1}\left(B, \mathbb{G}_{m}(S)\right) .
$$

Proof. Given $L \in \operatorname{Pic}^{\tau}(X)$, any restriction $L_{\tau}=q_{\tau}^{*}(L)$ is a numerically trivial line bundle on a toric variety defined over an affine scheme, hence trivial. Choose isomorphisms $h_{\tau}: L_{\tau} \rightarrow \mathcal{O}_{X_{\tau} \times S}$ and, for $e \in \operatorname{Hom}(\tau, \sigma)$, consider the composition

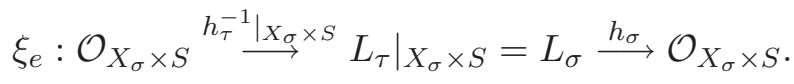

This is a locally invertible element of $\Gamma\left(X_{\sigma} \times S, \mathcal{O}_{X_{\sigma}} \times S\right)=A$, that is, $\xi_{e} \in \mathbb{G}_{m}(S)$. For a composition $\omega \stackrel{f}{\rightarrow} \tau \stackrel{e}{\rightarrow} \sigma$ it holds $\xi_{f \circ e}=\left.\xi_{f}\right|_{X_{\sigma} \times S} \cdot \xi_{e}$. Hence $\left(\xi_{e}\right)$ is a simplicial one-cocycle for the triangulation $\operatorname{Bar}(\mathscr{P})$ of $B$. Different choices of $h_{\tau}$ yield cohomologous $\left(\xi_{e}\right)$. 
Conversely, given $\xi=\left(\xi_{e}\right)$ we can enrich the gluing functor $F_{S, \bar{s}}$ from Definition 2.11 for the closed gluing data $\bar{s}$ associated to $s$ to also take care of a line bundle. The corresponding functor $G_{S, \bar{s}, \xi}$ maps $\tau \in \mathscr{P}$ to the pair $\left(X_{\tau}, \mathcal{O}_{X_{\tau} \times S}\right)$, and $e \in \operatorname{Hom}(\tau, \sigma)$ maps to the morphism $F_{S, \bar{s}}(e)$ of spaces and to the morphism

$$
\xi_{e} \cdot F_{S, \bar{s}}(e)^{*}: F_{S, \bar{s}}(e)^{-1}\left(\mathcal{O}_{X_{\tau} \times S}\right) \longrightarrow \mathcal{O}_{X_{\sigma}}
$$

of line bundles. In view of the methods presented in this section it is then clear that $G_{S, \bar{s}, \xi}$ has a limit $\left(X_{0}(B, \mathscr{P}, s), L\right)$ in the category consisting of pairs of an algebraic space and a line bundle, and that $L$ maps to $\xi \in H^{1}\left(B, \mathbb{G}_{m}(S)\right)$ under the map described above. Details are left to the reader.

q.e.d.

We end this section by discussing dualizing sheaves on $X=X_{0}(B$, $\mathscr{P}, s)$. Now we do not wish to go into the question of duality for algebraic spaces, which is a question which does not appear to be dealt with in the literature. Therefore, for the discussion which follows, assume that either duality does hold, or that $X$ is a scheme. This occurs as in Remark 2.19, or if $X$ arises through gluing in the cone picture, so that $X$ is in fact a projective scheme. In any event, we take $S=\operatorname{Spec} k, k$ an algebraically closed field. Note that $X$ is Gorenstein: it is covered by étale sets of the form $V(\sigma) \subseteq U(\sigma)$, and $V(\sigma)$ is Gorenstein by [38], pg. 126, Ishida's criteria, (3). Thus there is a dualizing sheaf $\omega_{X}$ which is in fact a line bundle. In addition, consider the map $\nu: \tilde{X}=\coprod_{v \in \mathscr{P}} X_{v} \rightarrow X$ (induced by $q_{v}$ on $X_{v}$ ). Then $\nu$ is in fact the normalization of $X$. Let $C \subseteq \tilde{X}$ be the conductor locus. Then by [41], Proposition $2.3, \nu^{*} \omega_{X} \cong \omega_{\tilde{X}}(C)$, where the latter denotes the "S $S_{2}$-isation" of $\omega_{\tilde{X}} \otimes \mathcal{O}_{\tilde{X}}(C)$; on $\tilde{X} \backslash \operatorname{Sing}(\tilde{X})$, this is just $\omega_{\tilde{X} \backslash \operatorname{Sing}(\tilde{X})}(C)$. Now by construction $C \cap X_{v}$ is just the reduced union of toric divisors of $X_{v}$, and this coincides on $X_{v} \backslash \operatorname{Sing}\left(X_{v}\right)$ with the anti-canonical class of $X_{v}$ (see e.g. [38], Corollary 3.3). Thus $\omega_{\tilde{X} \backslash \operatorname{Sing}(\tilde{X})}(C)=\mathcal{O}_{\tilde{X} \backslash \operatorname{Sing}(\tilde{X})}$, and $\omega_{\tilde{X}}(C)=\mathcal{O}_{\tilde{X}}$. We shall use this to calculate $\omega_{X}$ :

Theorem 2.39. Let $B$ be a compact integral affine manifold with singularities with a toric polyhedral decomposition $\mathscr{P}$, and s open gluing data over $S=$ Spec $k, k$ an algebraically closed field. Let $X=$ $X_{0}(B, \mathscr{P}, s)$. Suppose $X$ is a scheme, or duality holds as above. Then $\omega_{X}=\mathcal{O}_{X}$ if and only if $B$ is orientable, and $\omega_{X}^{\otimes 2}=\mathcal{O}_{X}$ if $B$ is not orientable.

Proof. First suppose $B$ is orientable. Then if $n=\operatorname{dim} B, H^{n}\left(X, \mathcal{O}_{X}\right)$ $=H^{n}(B, k)=k$ by orientability, compactness and Proposition 2.37. But by Serre duality, (using the hypothesis that $B$ is compact so that $X$ is proper over $k$ ) we then have $H^{0}\left(X, \omega_{X}\right)=k$. Thus we have a non-zero global section of $\omega_{X}$. To see that it is nowhere vanishing, use $\nu^{*} \omega_{X}=\mathcal{O}_{\tilde{X}}$. From this we conclude that a non-zero section of 
$\omega_{X}$ does not vanish on any $X_{v}$, and hence vanishes nowhere. Thus $\omega_{X} \cong \mathcal{O}_{X}$. Clearly, if $B$ is not orientable, then $H^{0}\left(X, \omega_{X}\right)=0$, so $\omega_{X}$ cannot be trivial. However, $B$ has an oriented unramified double cover $B^{\prime} \rightarrow B$. We can pull the affine structure on $B$ back to $B^{\prime}$, and also pull back $\mathscr{P}$ and $s$ to $B^{\prime}$ giving a polyhedral decomposition $\mathscr{P}^{\prime}$ of $B^{\prime}$ and open gluing data $s^{\prime}$, and then we obtain an étale double cover $\pi: X^{\prime}=X_{0}\left(B^{\prime}, \mathscr{P}^{\prime}, s^{\prime}\right) \rightarrow X$. Then $\pi^{*} \omega_{X}=\omega_{X^{\prime}} \cong \mathcal{O}_{X^{\prime}}$ since $B^{\prime}$ is orientable. From this it follows that $\omega_{X}$ must be two-torsion. q.e.d.

\section{Logarithmic structures}

3.1. Introduction to log structures. For a normal crossing divisor $X$ in a smooth variety $V$ there is the classical notion of differential form with logarithmic singularities along $X$. They generate an $\mathcal{O}_{V}$-module $\Omega_{V}^{1}(\log X)$. An important application is for a semistable degeneration $\pi: V \rightarrow \Delta$, which is a proper map from a smooth variety $V$ to a smooth curve $\Delta$ with $X=\pi^{-1}(0)$ a divisor with normal crossings and $\left.\pi\right|_{V \backslash X}$ smooth. One can then define the sheaf of $\log$ differentials $\Omega_{X}^{1}(\log X)$ by restricting $\Omega_{V}^{1}(\log X)$ to $V$ and dividing out by dlog $t$, where $t$ is the pull-back of a coordinate on $\Delta$. Steenbrink has shown that in this case $h^{q}\left(X, \Omega_{X}^{p}(\log X)\right)$ equals $h^{p, q}$ of a general fibre of $\pi[\mathbf{4 7}]$. Logarithmic (log-) geometry provides a means to define $\Omega_{X}^{p}(\log X)$ without knowledge of $V$. The fundamental notions of log geometry are due to Fontaine, Illusie and K. Kato. Standard references are [28],[27].

Before giving the definition let us list our conventions. All sheaves are understood in the étale topology. A monoid is a set with an associative product with a unit. We tacitly assume all monoids to be commutative. If $M$ is a monoid then $M^{\text {gp }}$ denotes the Grothendieck group associated to $M$; this is an abelian group together with a homomorphism $M \rightarrow$ $M^{\text {gp }}$ that has the universal property with respect to such maps. The group of invertible elements of $M$ is denoted $M^{\times}$. A monoid is integral if $M \rightarrow M^{\text {gp }}$ is injective; and an integral monoid is saturated if $x \in$ $M^{\text {gp }}, x^{n} \in M$ implies $x \in M$. A finitely generated, integral monoid is called fine. All the finitely generated monoids we are concerned about are toric, which by definition means finitely generated, saturated and $M^{\text {gp }}$ free. These are precisely the monoids isomorphic to $\mathbb{Z}^{n} \cap \sigma^{\vee}$ with $\sigma \subset \operatorname{Hom}\left(\mathbb{Z}^{n}, \mathbb{R}\right)$ a rational, strictly convex polyhedral cone.

Definition 3.1. A $\log$ structure on an algebraic space $X$ is a homomorphism

$$
\alpha_{X}: \mathcal{M}_{X} \longrightarrow \mathcal{O}_{X}
$$

of sheaves of (multiplicative) monoids such that $\alpha_{X}: \alpha_{X}^{-1}\left(\mathcal{O}_{X}^{\times}\right) \rightarrow \mathcal{O}_{X}^{\times}$ is an isomorphism. The triple $\left(X, \mathcal{M}_{X}, \alpha_{X}\right)$ is then called a log scheme. The quotient of $\mathcal{M}_{X}$ by the subsheaf $\mathcal{O}_{X}^{\times} \subset \mathcal{M}_{X}$ is the ghost sheaf $\overline{\mathcal{M}}_{X}$ of the $\log$ structure, with monoid structure written additively. 
A morphism of $\log$ spaces $F:\left(X, \mathcal{M}_{X}\right) \rightarrow\left(Y, \mathcal{M}_{Y}\right)$ consists of a morphism $\underline{F}: X \rightarrow Y$ of the underlying spaces together with a homomorphism $F^{\#}: \underline{F}^{-1}\left(\mathcal{M}_{Y}\right) \rightarrow \mathcal{M}_{X}$ commuting with the structure homomorphisms: $\alpha_{X} \circ F^{\#}=\underline{F}^{*} \circ \alpha_{Y}$.

By abuse of notation we often refer to $\mathcal{M}_{X}$ alone as a log structure.

Here is the prime example. All of our log structures are locally derived from this example.

Example 3.2. Let $X$ be a scheme and $D \subset X$ a closed subset of pure codimension 1 and $j: X \backslash D \rightarrow X$ the embedding of the complement. Then the natural inclusion

$$
\alpha_{X}: \mathcal{M}_{(X, D)}=j_{*}\left(\mathcal{O}_{X \backslash D}^{\times}\right) \cap \mathcal{O}_{X} \longrightarrow \mathcal{O}_{X}
$$

of the sheaf of regular functions with zeros contained in $D$ is a log structure on $X$. The monoid structure is multiplication.

If $X$ is locally Noetherian and normal the stalk of $\mathcal{M}_{X}:=\mathcal{M}_{(X, D)}$ at a geometric point $\bar{x} \rightarrow X$ has the following form. Let $r$ be the number of irreducible components of $D$ at $\bar{x}$. There is a map

$$
q: \mathcal{M}_{X, \bar{x}} \longrightarrow \mathbb{N}^{r}
$$

given by associating to a regular function $f \in \mathcal{M}_{X, \bar{x}}$ the vanishing orders $\operatorname{ord}_{\mu}(f)$ of $f$ along the components of $D$ at $\bar{x}$. This map factors over $\mathcal{M}_{X, \bar{x}} / \mathcal{O}_{X, \bar{x}}^{\times}$. On the other hand, if $f_{1}, f_{2} \in \mathcal{M}_{X, \bar{x}} \subset \mathcal{O}_{X, \bar{x}}$ have the same vanishing orders along $D$ then by normality $f_{2}=h \cdot f_{1}$ for some $h \in \mathcal{O}_{X, \bar{x}}^{\times}$. This shows that $\overline{\mathcal{M}}_{X, \bar{x}}=\mathcal{M}_{X, \bar{x}} / \mathcal{O}_{X, \bar{x}}^{\times}$is a submonoid of $\left(\mathbb{N}^{r},+\right)$. To see that it is finitely generated introduce the componentwise partial order on $\mathbb{N}^{r}$, that is $\left(a_{1}, \ldots, a_{r}\right) \geq\left(b_{1}, \ldots, b_{r}\right)$ if $a_{i} \geq b_{i}$ for all $i$. Then $f, g \in \mathcal{M}_{X, \bar{x}}$ and $q(f) \geq q(g)$ implies $f / g \in \mathcal{M}_{X, \bar{x}}$, again by normality. Therefore $\operatorname{im}(q)$ is generated by minimal elements. Using the fact that for any $a \in \mathbb{N}^{r}$ the set $\left\{b \in \mathbb{N}^{r} \mid b \leq a\right\}$ is finite, one can show any infinite sequence in $\mathbb{N}^{r}$ contains an increasing subsequence. Therefore the set of minimal elements in $\operatorname{im}(q)$ is finite.

In particular, $\overline{\mathcal{M}}_{X, \bar{x}}^{\mathrm{gp}}$ is a finitely generated subgroup of $\mathbb{Z}^{r}$, and then $\operatorname{Ext}^{1}\left(\overline{\mathcal{M}}_{X, \bar{x}}^{\mathrm{gp}}, \mathcal{O}_{X, \bar{x}}^{\times}\right)$is trivial. Hence the sequence

$$
1 \longrightarrow \mathcal{O}_{X, \bar{x}}^{\times} \longrightarrow \mathcal{M}_{X, \bar{x}}^{\mathrm{gp}} \longrightarrow \overline{\mathcal{M}}_{X, \bar{x}}^{\mathrm{gp}} \longrightarrow 0
$$

splits (non-canonically) and

$$
\mathcal{M}_{X, \bar{x}}=\overline{\mathcal{M}}_{X, \bar{x}} \times \overline{\mathcal{M}}_{X, \bar{x}}^{\mathrm{gp}} \mathcal{M}_{X, \bar{x}}^{\mathrm{gp}}=\overline{\mathcal{M}}_{X, \bar{x}} \oplus \mathcal{O}_{X, \bar{x}}^{\times} .
$$

We emphasize that this splitting does not, however, hold on the sheaf level. For example, let $k$ be a field and consider $X=\boldsymbol{A}_{k}^{1}$ and $D=\{0\}$. Then $\overline{\mathcal{M}}_{X}$ is the skyscraper sheaf $\mathbb{N}_{\{0\}}$. Thus $\overline{\mathcal{M}}_{X} \oplus \mathcal{O}_{X}^{\times}$has nontrivial sections with support in $\{0\}$, while this is not true for $\mathcal{M}_{X} \subset$ 
$\mathcal{O}_{X}$. The reason is that unlike for coherent sheaves the canonical map $\mathcal{H o m}\left(\overline{\mathcal{M}}_{X}, \mathcal{O}_{X}^{\times}\right)_{\bar{x}} \rightarrow \operatorname{Hom}\left(\overline{\mathcal{M}}_{X, \bar{x}}, \mathcal{O}_{X, \bar{x}}^{\times}\right)$is not bijective at $x=0$.

If $X$ is not normal the stalks of $\overline{\mathcal{M}}_{X}$ might not be finitely generated nor even countable. Here is an example that we learned from A. Ogus. Let $X=\operatorname{Spec} \mathbb{C}[x, y] /\left(x^{2}-y^{3}\right)$ and $D=V(x, y)$. Then for any $a \in \mathbb{C}$ the function $x-a y$ lies in $\mathcal{M}_{(X, D)}$, but if $a, b \in \mathbb{C}$ the existence of $h \in \mathbb{C} \llbracket x, y \rrbracket /\left(x^{2}-y^{3}\right)$ with $x-a y=h \cdot(x-b y)$ formally, implies $a=b$. The same works for a node $X=\operatorname{Spec} \mathbb{C}[x, y] /(x y)$.

For later use, we include

Lemma 3.3. If $X$ is normal and $D \subset X$ is the support of an effective Cartier divisor then $\mathcal{M}_{(X, D)}^{\mathrm{gp}}=j_{*} \mathcal{O}_{X \backslash D}^{\times}$where $j: X \backslash D \rightarrow X$ is the inclusion.

Proof. The universal property of the morphism to the associated Grothendieck group gives a homomorphism

$$
\mathcal{M}_{(X, D)}^{\mathrm{gp}} \longrightarrow j_{*} \mathcal{O}_{X \backslash D}^{\times}, \quad(f, g) \longmapsto f / g, \quad f, g \in j_{*} \mathcal{O}_{X \backslash D}^{\times} \cap \mathcal{O}_{X},
$$

which is clearly injective. Conversely, let $\bar{x} \rightarrow X$ be a geometric point and $h \in\left(j_{*} \mathcal{O}_{X \backslash D}^{\times}\right)_{\bar{x}}$. Let $t \in \mathcal{O}_{X, \bar{x}}$ be a function defining at $\bar{x}$ a Cartier divisor with support $D$. If $n \geq 0$ is the maximum of the pole orders of $h$ along the prime components of $D$ at $\bar{x}$ then $t^{n} h$ extends in codimension one to $X$. By normality of $X$ it follows that $f=t^{n} h \in \mathcal{O}_{X, \bar{x}}$. Then $f, t^{n} \in \mathcal{M}_{X, \bar{x}}$ and $\left(f, t^{n}\right) \in \mathcal{M}_{(X, D)}^{\mathrm{gp}}$ maps to $h . \quad$ q.e.d.

The log structures obtained in this way can still be quite pathological. For example, $\mathcal{M}_{X}$ might not be locally generated by global sections, see Example 3.6. This leads to a certain coherence condition that requires some preparation to explain.

Definition 3.4. An arbitrary homomorphism of sheaves of monoids

$$
\varphi: \mathcal{P} \longrightarrow \mathcal{O}_{X}
$$

defines an associated log structure $\mathcal{M}_{X}$ by

$$
\mathcal{M}_{X}=\left(\mathcal{P} \oplus \mathcal{O}_{X}^{\times}\right) /\left\{\left(p, \varphi(p)^{-1}\right) \mid p \in \varphi^{-1}\left(\mathcal{O}_{X}^{\times}\right)\right\},
$$

and $\alpha_{X}(p, h)=h \cdot \varphi(p)$.

If $f: X \rightarrow Y$ is a morphism of algebraic spaces, the pull-back of a $\log$ structure $\mathcal{M}_{Y}$ on $Y$ to $X$ is the $\log$ structure associated to

$$
f^{*} \circ \alpha_{Y}: f^{-1} \mathcal{M}_{Y} \longrightarrow \mathcal{O}_{X} .
$$

The notation is $f^{*} \mathcal{M}_{Y}$. If $f$ is an embedding we also speak of restricting the log structure to $X$. 
Example 3.5. For any field $k$ and monoid $Q$ (written additively) with $Q^{\times}=\{0\}$ let $\mathcal{M}_{\text {Spec } k}$ be the monoid sheaf with sections $Q \times K^{\times}$ over a separable extension $k \subset K$. Then

$$
Q \times K^{\times} \longrightarrow K, \quad(q, a) \longmapsto \begin{cases}a, & q=0 \\ 0, & \text { otherwise },\end{cases}
$$

defines a homomorphism $\mathcal{M}_{\text {Spec } k} \rightarrow \mathcal{O}_{\text {Spec } k}$, which is a log structure. Special cases are $Q=0$ and $Q=\mathbb{N}$, which give the trivial and standard log points respectively. The notation for the standard log point is Spec $k^{\dagger}$. This is the restriction of the log structure associated to the origin $o \subset \mathbb{A}_{k}^{1}$ to $o$.

Another case is when $\mathcal{P}=P$ is a constant sheaf with stalks a fine monoid, also denoted $P$. Then $\varphi$ is a chart for its associated log structure. More generally, a chart for a log structure $\alpha_{X}: \mathcal{M}_{X} \rightarrow \mathcal{O}_{X}$ is a homomorphism $\varphi: P \rightarrow \mathcal{O}_{X}$ and a factorization of $\varphi$ via $\alpha_{X}$, inducing an isomorphism of the $\log$ structure associated to $\varphi$ with $\mathcal{M}_{X}$. A log structure possessing charts étale locally is called fine. In general it is impossible to find a global chart for a fine log structure. This is the concept of coherence for $\mathcal{M}_{X}$. At least in the fine saturated case there exists then for every geometric point $\bar{x} \rightarrow X$ a chart in an étale neighbourhood with $P=\overline{\mathcal{M}}_{X, \bar{x}}$, see [39] Lemma 2.12. Another way to think of charts is by noting that $P \rightarrow \mathcal{O}_{X}$ defines, étale locally, a morphism to an affine toric variety:

$$
\Phi: X \longrightarrow \operatorname{Spec} \mathbb{Z}[P] .
$$

Now Spec $\mathbb{Z}[P]$ has a distinguished log structure defined either as associated to the canonical map $P \rightarrow \mathcal{O}_{\text {Spec } \mathbb{Z}[P]}$, or by the log structure coming from the divisor defined by the complement of the big torus orbit as in Example 3.2. Then $\mathcal{M}_{X}$ is isomorphic to $\Phi^{*} \mathcal{M}_{\text {Spec } \mathbb{Z}[P]}$. In particular, toric varieties provide an ample source of fine log structures.

Example 3.6. Here is an example of a naturally occuring log structure that is not fine. Let $k$ be an algebraically closed field and $X=$ Spec $k[P]$ with $P$ the monoid generated by $e_{1}, \ldots, e_{4}$ with single relation $e_{1}+e_{3}=e_{2}+e_{4}$. This is the 3-dimensional $A_{1}$-singularity $x y-t w=0$ in $\boldsymbol{A}_{k}^{4}$ written as a toric variety. Let $D$ be the divisor given by $t=0$, a union of two copies of $\boldsymbol{A}_{k}^{2}$. Then the $\log$ structure on $X$ associated to $D$ is not fine at the zero-dimensional torus orbit $o \in X$ : If $f \in \mathcal{O}_{X, \bar{o}}$ has zero locus contained in $D$ then $f=h \cdot t^{n}$ with $h \in \mathcal{O}_{X, \bar{o}}^{\times}$. Therefore

$$
\overline{\mathcal{M}}_{X, \bar{o}}=\mathbb{N} \text {. }
$$

On the other hand, at the generic point $\eta$ of $x=y=0$ the divisor $D$ is normal crossing with two components. Hence

$$
\overline{\mathcal{M}}_{X, \bar{\eta}}=\mathbb{N}^{\oplus 2} .
$$


Since $o \in \operatorname{cl}(\eta)$ this shows that $\overline{\mathcal{M}}_{X}$ is not locally generated by global sections, which holds for fine log structures. This is not a perverse example, but a fundamental issue in our approach. In the example of degeneration of quartics given in the introduction, the total space $\mathcal{X}$ has 24 ordinary double points, and locally the structure of $\mathcal{X}_{0} \subseteq \mathcal{X}$ is exactly as described here.

The example also illustrates that the obstruction to being fine might already be captured by the ghost sheaf of the log structure. This is indeed the case as we will see instantly. Let us say a sheaf of monoids $\overline{\mathcal{M}}$ is fine if its stalks are fine monoids and if for every geometric point $\bar{x} \rightarrow X$ there exists an étale neighbourhood $f: U \rightarrow X$ of $\bar{x}$ and a surjection from a constant sheaf

$$
P \longrightarrow f^{-1} \overline{\mathcal{M}}
$$

which is an isomorphism on stalks at $\bar{x}$. We have the following criterion.

Proposition 3.7. A log structure is fine iff its ghost sheaf is fine.

Proof. Clearly if the log structure is fine, so is the ghost sheaf. Conversely, suppose the ghost sheaf is fine. By the same argument as in Example 3.2 the exact sequence

$$
1 \longrightarrow \mathcal{O}_{X, \bar{x}}^{\times} \longrightarrow \mathcal{M}_{X, \bar{x}}^{\mathrm{gp}} \longrightarrow \overline{\mathcal{M}}_{X, \bar{x}}^{\mathrm{gp}} \longrightarrow 0
$$

splits, inducing a right inverse $\kappa$ of $\mathcal{M}_{X, \bar{x}} \rightarrow \overline{\mathcal{M}}_{X, \bar{x}}$. Put $P=\overline{\mathcal{M}}_{X, \bar{x}}$. Since $\overline{\mathcal{M}}_{X}$ is a fine monoid sheaf, after going over to an étale neighbourhood of $\bar{x}$ there exists a surjection $\psi: P \rightarrow \overline{\mathcal{M}}_{X}$. Composing with $\kappa$ gives a sheaf homomorphism (in a smaller neighbourhood)

$$
\varphi: P \longrightarrow \mathcal{M}_{X},
$$

which is a chart because it induces a surjection to the ghost sheaf. In fact, for a geometric point $\bar{y} \rightarrow X$ and $m \in \mathcal{M}_{X, \bar{y}}$ let $\bar{m}$ be the image in $\overline{\mathcal{M}}_{X, \bar{y}}$. There exists $p \in P$ with $\psi(p)=\bar{m}$. Then $\varphi(p)=h \cdot m$ for $h \in \mathcal{O}_{X, \bar{y}}^{\times}$. This shows that the map $P \oplus \mathcal{O}_{X}^{\times} \rightarrow \mathcal{M}_{X}$ induced by $\varphi$ is surjective. The kernel is the submonoid of pairs $(p, h)$ with $\alpha_{X}(\varphi(p)) \cdot h=1$, which coincides with the submonoid divided out in the definition of the associated log structure.

q.e.d.

A central concept in log geometry is (log) smoothness. The definition runs analogously to formal smoothness for schemes. For our purposes it is more instructive to use the characterization of log smooth morphisms due to K. Kato [28] Theorem 3.5, see also [27] Theorem 4.1. A model log smooth map is given by the map of log schemes induced by a morphism of monoids $Q \rightarrow P$ with finite kernel, with some subtlety in non-zero characteristic. 
Definition 3.8. A morphism $f:\left(X, \mathcal{M}_{X}\right) \rightarrow\left(Y, \mathcal{M}_{Y}\right)$ of fine log schemes is $\log$ smooth if étale locally it fits into a commutative diagram of the form

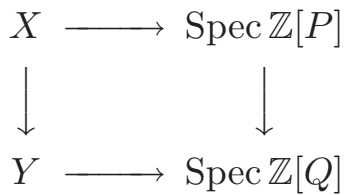

with the following properties.

1) The horizontal arrows induce charts for the log structures on $X$ and $Y$ that are compatible with $f^{\#}: f^{-1} \mathcal{M}_{Y} \rightarrow \mathcal{M}_{X}$.

2) The induced morphism $X \rightarrow Y \times_{\operatorname{Spec} \mathbb{Z}[Q]} \operatorname{Spec} \mathbb{Z}[P]$ is a smooth morphism of schemes.

3) The right-hand vertical arrow comes from a monoid homomorphism $Q \rightarrow P$ with

$$
\operatorname{ker}\left(Q^{\mathrm{gp}} \longrightarrow P^{\mathrm{gp}}\right)
$$

and the torsion part of $\operatorname{coker}\left(Q^{\mathrm{gp}} \rightarrow P^{\mathrm{gp}}\right)$ finite groups of orders invertible on $X$.

If the characteristic is 0 and all monoids are toric, then the last condition just requires the homomorphism $Q \rightarrow P$ to be injective.

Obviously any toric morphism between affine toric varieties induced by a monoid homomorphism $Q \rightarrow P$ as in (3) is log smooth. In particular, log smooth morphisms need not be flat, a toric blowup for example. This will only be the case if we also ask $f$ to be integral, which by definition means that for every geometric point $\bar{x} \rightarrow X$ the ring homomorphism $\mathbb{Z}\left[\overline{\mathcal{M}}_{Y, f(\bar{x})}\right] \rightarrow \mathbb{Z}\left[\overline{\mathcal{M}}_{X, \bar{x}}\right]$ is flat.

Given a log smooth morphism it is sometimes useful to consider certain adapted charts. An obstruction for finding such charts in positive characteristic is torsion in $\mathcal{M}_{X}^{\mathrm{gp}} / f^{*} \mathcal{M}_{Y}^{\mathrm{gp}}=\overline{\mathcal{M}}_{X}^{\mathrm{gp}} / f^{-1} \overline{\mathcal{M}}_{Y}^{\mathrm{gp}}$. In our cases torsion never occurs and the following proposition suffices.

Proposition 3.9 ([39] Proposition 2.25). Let $f:\left(X, \mathcal{M}_{X}\right) \rightarrow$ $\left(Y, \mathcal{M}_{Y}\right)$ be a log smooth morphism of fine log schemes. Then for every geometric point $\bar{x} \rightarrow X$ with $\mathcal{M}_{Y, f(\bar{x})}^{\mathrm{gp}} \rightarrow \mathcal{M}_{X, \bar{x}}^{\mathrm{gp}}$ injective with torsion-free cokernel, any chart $\varphi: Y \rightarrow \operatorname{Spec} \mathbb{Z}\left[\overline{\mathcal{M}}_{Y, f(\bar{x})}\right]$ inducing an isomorphism of ghost sheaves at $f(\bar{x})$ fits into a diagram

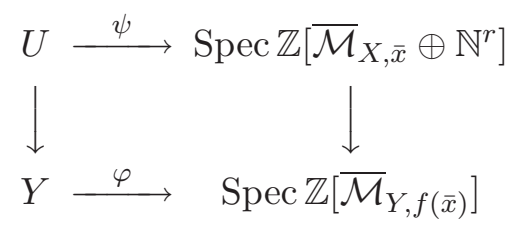

with the right-hand vertical arrow defined by the composition of $f_{\bar{x}}^{\#}$ : $\overline{\mathcal{M}}_{Y, f(\bar{x})} \rightarrow \overline{\mathcal{M}}_{X, \bar{x}}$ with the inclusion (id,0) $: \overline{\mathcal{M}}_{X, \bar{x}} \rightarrow \overline{\mathcal{M}}_{X, \bar{x}} \oplus \mathbb{N}^{r}$, and 
$\psi$ étale with the induced map $\overline{\mathcal{M}}_{X, \bar{x}} \rightarrow \mathcal{O}_{U}$ defining a chart for $\left(X, \mathcal{M}_{X}\right)$ on an étale neighbourhood $U \rightarrow X$ of $\bar{x}$.

The factor $\mathbb{N}^{r}$ is necessary if one insists on $\psi$ to be étale rather than smooth. (Consider the example of a smooth morphism with trivial log structures.) However, this factor has no effect on the log structure.

Log smoothness extends the class of smooth objects considerably:

\section{Example 3.10.}

1) Any toric variety over a scheme $S$ with its canonical log structure is $\log$ smooth over $S$, viewed as log scheme with trivial log structure.

2) Let $\pi: V \rightarrow \Delta$ be a semistable family of algebraic varieties as in the first paragraph of this section and $x \in \Delta$ a closed point with separably closed residue field. $\pi$ induces a log morphism for the $\log$ structures associated to the divisors $\{x\} \subset \Delta$ and $X=\pi^{-1}(x) \subset V$ introduced in Example 3.2. Étale locally and up to smooth factors it has the form Spec $k\left[\mathbb{N}^{r}\right] \rightarrow$ Spec $k[\mathbb{N}]$ induced by the diagonal morphism $\mathbb{N} \rightarrow \mathbb{N}^{r}$. Hence it is $\log$ smooth and integral.

3) Restricting the previous example to the central fibre gives another interesting log-smooth morphism $\left(X, \mathcal{M}_{X}\right) \rightarrow \operatorname{Spec} k(x)^{\dagger}$. More generally one can show that a variety $X$ with normal crossings supports a $\log$ smooth morphism to the standard log point if $X$ is $d$-semistable, which by definition means $\mathcal{E} x t^{1}\left(\Omega_{X}^{1}, \mathcal{O}_{X}\right) \cong \mathcal{O}_{X_{\text {sing }}}$ $([\mathbf{1 2}],[\mathbf{2 9}],[\mathbf{2 7}])$. So the existence of a $\log$ smooth structure restricts not only the type of singularities of $X$, but poses also some more subtle global analytical conditions (here the triviality of a locally free sheaf over $X_{\text {sing }}$ ).

3.2. Sheaves of log structures. We next study moduli of log structures on a space with given fine ghost sheaf $\overline{\mathcal{M}}_{X}$. For the following discussion we make the overall assumption that $X$ is reduced. We first show that the structure homomorphism $\alpha: \mathcal{M}_{X} \rightarrow \mathcal{O}_{X}$ is then already determined by the extension

$$
1 \longrightarrow \mathcal{O}_{X}^{\times} \longrightarrow \mathcal{M}_{X}^{\mathrm{gp}} \stackrel{q}{\longrightarrow} \overline{\mathcal{M}}_{X}^{\mathrm{gp}} \longrightarrow 0 .
$$

Proposition 3.11. Let $X$ be a reduced algebraic space. Let $\alpha, \alpha^{\prime}$ : $\mathcal{M}_{X} \rightarrow \mathcal{O}_{X}$ be two fine log structures on $X$ with the same monoid sheaf and such that $\alpha^{\prime} \circ \alpha^{-1}: \mathcal{O}_{X}^{\times} \rightarrow \mathcal{O}_{X}^{\times}$is the identity. Then $\alpha=\alpha^{\prime}$.

Proof. Let $\bar{\eta}$ be a generic geometric point of $X$. Let $m \in \mathcal{M}_{X, \bar{\eta}}$. Since $\mathcal{O}_{X, \bar{\eta}}$ is a field $\alpha(m)$ is either 0 or invertible. The first case occurs if $q(m) \neq 0$, and then also $\alpha^{\prime}(m)=0$. In the invertible case the assumption implies that $\alpha(m)=\alpha^{\prime}(m)$. Thus in any case $\alpha(m)=\alpha^{\prime}(m)$. Therefore $\alpha$ and $\alpha^{\prime}$ agree generically, and $X$ being reduced this implies $\alpha=\alpha^{\prime}$.

q.e.d. 
Corollary 3.12. For a reduced algebraic space $X$ the set of isomorphism classes of log structures with ghost sheaf $\overline{\mathcal{M}}_{X}$ is a subset of $\operatorname{Ext}^{1}\left(\overline{\mathcal{M}}_{X}^{\mathrm{gp}}, \mathcal{O}_{X}^{\times}\right)$.

By abuse of notation we will therefore confuse an isomorphism class of log structures with its associated extension class. However, it is not true that all of $\operatorname{Ext}^{1}\left(\overline{\mathcal{M}}_{X}^{\mathrm{gp}}, \mathcal{O}_{X}^{\times}\right)$arises in this way. Let us study this in one example.

Example 3.13. Let $k$ be a separably closed field. Consider the quadruple point $X=\operatorname{Spec} k\left[x_{1}, \ldots, x_{4}\right] /\left(x_{1} x_{3}, x_{2} x_{4}\right)$ with $\overline{\mathcal{M}}_{X}$ coming from the $\log$ structure induced by embedding into $\operatorname{Spec} k\left[x_{1}, \ldots, x_{4}\right]$ / $\left(x_{1} x_{3}-x_{2} x_{4}\right)=\operatorname{Spec} k[P]$ with

$$
P=\left\langle e_{1}, \ldots, e_{4} \mid e_{1}+e_{3}=e_{2}+e_{4}\right\rangle .
$$

Denote by $o \in X$ the zero-dimensional torus orbit. This is the unique point with $\overline{\mathcal{M}}_{X, \bar{o}}=P$. Note that any element of $P$ extends uniquely to a global section of $\overline{\mathcal{M}}_{X}$, and these global sections generate $\overline{\mathcal{M}}_{X}$ at every point.

Any extension $q: \mathcal{M}_{X} \rightarrow \overline{\mathcal{M}}_{X}$ by $\mathcal{O}_{X}^{\times}$gives four $\mathcal{O}_{X}^{\times}$-torsors

$$
\mathcal{L}_{i}=q^{-1}\left(e_{i}\right) .
$$

There is an identification of $\mathcal{O}_{X}^{\times}$-torsors

$$
\left(\mathcal{L}_{1} \times \mathcal{L}_{3}\right) / \mathcal{O}_{X}^{\times}=q^{-1}\left(e_{1}+e_{3}\right)=q^{-1}\left(e_{2}+e_{4}\right)=\left(\mathcal{L}_{2} \times \mathcal{L}_{4}\right) / \mathcal{O}_{X}^{\times} .
$$

By abuse of notation we are going to use tensor notation for sections of the quotients on the left and right-hand sides. The components of $X$ are $X_{i}=V\left(x_{i+2}, x_{i+3}\right)$, the indices taken modulo 4 . Let $\eta_{i}$ be the generic point of $X_{i}$. Since $\left.q\left(\mathcal{L}_{i}\right)\right|_{X \backslash \operatorname{supp}\left(e_{i}\right)}=0$, on $X \backslash \operatorname{supp}\left(e_{i}\right)=$ $X \backslash\left(X_{i-2} \cup X_{i-3}\right) \subseteq X_{i-1} \cup X_{i}=V\left(x_{i+2}\right)$ the unit in $\mathcal{M}_{X}$ lies in $\mathcal{L}_{i}$. This gives a distinguished section $s_{i}$ of $\mathcal{L}_{i}$ over $X \backslash \operatorname{supp}\left(e_{i}\right)$. Obviously $s_{i}$ extends as the unit section of $\mathcal{O}_{X}^{\times} \subset \mathcal{M}_{X}$, but generally not as a section of $\mathcal{L}_{i}$. However, possibly after going over to an étale neighbourhood $U \rightarrow X$ of the origin, the $\mathcal{L}_{i}$ become trivial. Therefore $x_{i}^{d_{i}} \cdot s_{i}$ extends uniquely to a section $\sigma_{i}$ of $\left.\mathcal{L}_{i}\right|_{U}$ for some $d_{i} \in \mathbb{Z}$ depending only on the isomorphism class of the extension. Note that $x_{i}^{d_{i}} s_{i}$ restricts to $x_{i}^{d_{i}}$ on $X \backslash \operatorname{supp}\left(e_{i}\right)$. By the above identification there exists $h \in \mathcal{O}_{X, \bar{o}}^{\times}$with

$$
\sigma_{2} \otimes \sigma_{4}=h \cdot \sigma_{1} \otimes \sigma_{3} .
$$

Any other $\sigma_{i}^{\prime} \in \mathcal{L}_{i, \bar{o}}$ restricting to $x_{i}^{d_{i}}$ away from $\operatorname{supp}\left(e_{i}\right)$ has the form $\sigma_{i}^{\prime}=h_{i} \cdot \sigma_{i}$ with $h_{i} \in \mathcal{O}_{X, \bar{o}}^{\times}$and $\left.h_{i}\right|_{V\left(x_{i+2}\right)}=1$. Writing $h_{i}=1+x_{i+2} f_{i}$, this changes $h$ by $\left(1+x_{3} f_{1}\right)\left(1+x_{1} f_{3}\right)\left(1+x_{4} f_{2}\right)^{-1}\left(1+x_{2} f_{4}\right)^{-1}$. Terms of this form generate $1+\mathfrak{m}_{\bar{o}}$. In particular, the residue of $h$ in $k=\mathcal{O}_{X, \bar{o}} / \mathfrak{m}_{\bar{o}}$ is well-defined. We will see below that this residue together with the $d_{i}$ 
are the only invariants of the extension. Conversely, for any $\lambda \in k^{\times}$the pull-back of the standard log structure by

$$
\left(x_{1}, x_{2}, x_{3}, x_{4}\right) \longmapsto\left(\lambda \cdot x_{1}, x_{2}, x_{3}, x_{4}\right)
$$

defines an extension with $d_{1}, \ldots, d_{4}=1, h=\lambda$. So any residue is possible. Using results below, one can see that in fact

$$
\operatorname{Ext}^{1}\left(\overline{\mathcal{M}}_{X}^{\mathrm{gp}}, \mathcal{O}_{X}^{\times}\right)=\mathbb{Z}^{4} \oplus k^{\times} .
$$

For such an extension $\left(d_{i}, \lambda\right)$ there exists a homomorphism $\mathcal{M}_{X} \rightarrow \mathcal{O}_{X}$ inducing the identity on $\mathcal{O}_{X}^{\times}$iff $d_{i}>0$ for every $i$.

This example is quite typical: To define $\alpha_{X}$ on $m \in \mathcal{M}_{X, \bar{x}}$ let $\bar{\eta}$ be a geometric generic point of $X$ with $\bar{x} \in \operatorname{cl}(\bar{\eta})$. Then either $q(m)_{\bar{\eta}} \neq 0$ and we must have $\alpha_{X}(m)_{\bar{\eta}}=0$; or $q(m)_{\bar{\eta}}=0$ and $m \in \mathcal{O}_{X, \bar{\eta}}^{\times}$prescribes $\alpha_{X}(m)_{\bar{\eta}}$. The question is then if the regular function defined on the generic points with closure containing $\bar{x}$ extends as regular function to $\bar{x}$. In other words, the subspace of $\operatorname{Ext}^{1}\left(\overline{\mathcal{M}}_{X}^{\mathrm{gp}}, \mathcal{O}_{X}^{\times}\right)$parametrizing log structures with ghost sheaf $\overline{\mathcal{M}}_{X}$ is characterized by a pointwise positivity condition.

The next aim is an explicit description of isomorphism classes of log structures with given fine ghost sheaf, building on Corollary 3.12. Let $\mathcal{M}_{X} \rightarrow \mathcal{O}_{X}$ be a fine $\log$ structure on $X, \bar{x} \rightarrow X$ a geometric point and $P=\overline{\mathcal{M}}_{X, \bar{x}}$. After replacing $X$ by an étale open set, we may assume that there is a chart $P \rightarrow \mathcal{M}_{X}$. In particular, $P=\Gamma\left(X, \overline{\mathcal{M}}_{X}\right)$ and for every geometric point $\bar{y} \in X$ the restriction map $P \rightarrow \overline{\mathcal{M}}_{X, \bar{y}}$ is surjective. Define the relation sheaf $\mathcal{R}$ of $\overline{\mathcal{M}}_{X}^{\mathrm{gp}}$ for the given chart by

$$
0 \longrightarrow \mathcal{R} \longrightarrow P^{\mathrm{gp}} \longrightarrow \overline{\mathcal{M}}_{X}^{\mathrm{gp}} \longrightarrow 0 \text {. }
$$

Because $P^{\text {gp }}$ is a constant sheaf with stalks a free finitely generated abelian group, $\mathcal{E} x t^{1}\left(P^{\mathrm{gp}}, \mathcal{O}_{X}^{\times}\right)$vanishes as sheaf in the étale topology. We obtain an exact sequence

$$
\begin{aligned}
0 \longrightarrow \mathcal{H o m}\left(\overline{\mathcal{M}}_{X}^{\mathrm{gp}}, \mathcal{O}_{X}^{\times}\right) \longrightarrow \mathcal{H o m}\left(P^{\mathrm{gp}}, \mathcal{O}_{X}^{\times}\right) \\
\longrightarrow \mathcal{H o m}\left(\mathcal{R}, \mathcal{O}_{X}^{\times}\right) \longrightarrow \mathcal{E} x t^{1}\left(\overline{\mathcal{M}}_{X}^{\mathrm{gp}}, \mathcal{O}_{X}^{\times}\right) \longrightarrow 0 .
\end{aligned}
$$

Therefore

$$
\mathcal{E} x t^{1}\left(\overline{\mathcal{M}}_{X}^{\mathrm{gp}}, \mathcal{O}_{X}^{\times}\right)=\mathcal{H o m}\left(\mathcal{R}, \mathcal{O}_{X}^{\times}\right) / \mathcal{H} o m\left(P^{\mathrm{gp}}, \mathcal{O}_{X}^{\times}\right) .
$$

Now any $p \in P^{\text {gp }}$ induces a section of $\overline{\mathcal{M}}_{X}^{\text {gp }}$ that is zero on $X \backslash \operatorname{supp}(p)$. (When writing $\operatorname{supp}(p)$ we always mean the support of $p$ viewed as section of $\overline{\mathcal{M}}_{X}^{\text {gp }}$.) Thus $\left.p\right|_{X \backslash \operatorname{supp}(p)}$ is a section of $\mathcal{R}$, and these sections generate $\mathcal{R}$. Denoting $j_{p}: X \backslash \operatorname{supp}(p) \rightarrow X$ the inclusion, a more explicit description of the numerator is then

$$
\mathcal{H o m}\left(\mathcal{R}, \mathcal{O}_{X}^{\times}\right)_{\bar{x}}=\left\{\begin{array}{l|l}
\left(h_{p}\right)_{p \in P \mathrm{gp}} & \begin{array}{l}
h_{p} \in\left(j_{p_{*}} \mathcal{O}_{X \backslash \operatorname{supp}(p)}^{\times}\right)_{\bar{x}} \\
h_{p} \cdot h_{q}=h_{p+q} \text { on } X \backslash \operatorname{supp}(p+q)
\end{array}
\end{array}\right\} .
$$


As $\mathcal{H o m}\left(P^{\mathrm{gp}}, \mathcal{O}_{X}^{\times}\right)_{\bar{x}}=\operatorname{Hom}\left(\overline{\mathcal{M}}_{X, \bar{x}}^{\mathrm{gp}}, \mathcal{O}_{X, \bar{x}}^{\times}\right)$we obtain the following result.

Proposition 3.14. The stalk of $\mathcal{E} x t^{1}\left(\overline{\mathcal{M}}_{X}^{\mathrm{gp}}, \mathcal{O}_{X}^{\times}\right)$at a geometric point $\bar{x}$ of $X$ is canonically isomorphic to the quotient of

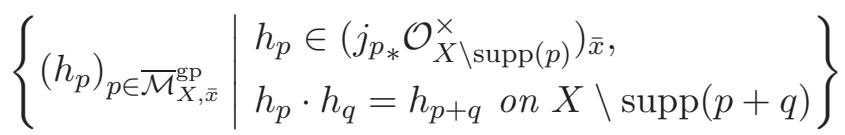

by $\operatorname{Hom}\left(\overline{\mathcal{M}}_{X, \bar{x}}^{\mathrm{gp}}, \mathcal{O}_{X, \bar{x}}^{\times}\right)$. The extension class of the log structure associated to a homomorphism $\varphi: \overline{\mathcal{M}}_{X, \bar{x}} \rightarrow \mathcal{O}_{X, \bar{x}}$ with $V(\varphi(p))=\operatorname{supp}(p)$ is represented by

$$
h_{p}=\left.\varphi(p)\right|_{X \backslash \operatorname{supp}(p)} .
$$

Conversely, $\left(h_{p}\right)_{p}$ comes from a log structure if $h_{p}$ extends by 0 to $X$ for all $p \in \overline{\mathcal{M}}_{X, \bar{x}}$.

Proof. It remains to prove the statement about log structures. Let $\tilde{\varphi}: P \rightarrow \mathcal{M}_{U}$ be the chart induced by $\varphi$ on an étale neighbourhood $U \rightarrow X$ of $\bar{x}$. Let $\mathcal{R}=\operatorname{ker}\left(P^{\mathrm{gp}} \rightarrow \overline{\mathcal{M}}_{U}^{\mathrm{gp}}\right)$ be the associated relation sheaf. The composition $\mathcal{R} \longrightarrow P^{\mathrm{gp}} \longrightarrow \mathcal{M}_{U}^{\mathrm{gp}}$ factors through $\mathcal{O}_{U}^{\times}$. The factorization sends the local section of $\mathcal{R}$ induced by $p \in P^{\mathrm{gp}}$ to $h_{p}=\left.\alpha_{X}(\tilde{\varphi}(p))\right|_{U \backslash \operatorname{supp}(p)}=\left.\varphi(p)\right|_{U \backslash \operatorname{supp}(p)} \in \mathcal{O}_{U \backslash \operatorname{supp}(p)}^{\times}$. This defines a map $\psi: \mathcal{R} \rightarrow \mathcal{O}_{U}^{\times}$. By definition $\psi$ makes the following diagram with exact rows commutative.

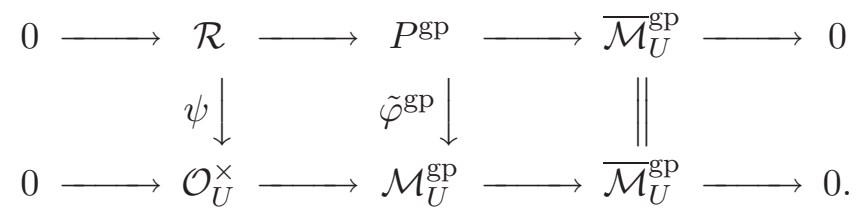

This shows that the class of the extension in the lower row defining the $\log$ structure is the image of $\psi$ under the connecting homomorphism $\operatorname{Hom}\left(\mathcal{R}, \mathcal{O}_{U}^{\times}\right) \rightarrow \operatorname{Ext}^{1}\left(\overline{\mathcal{M}}_{U}^{\mathrm{gp}}, \mathcal{O}_{U}^{\times}\right)$. Conversely, assume an extension $\tilde{h}_{p}$ of $h_{p}$ by zero exists. Then

$$
p \longmapsto \tilde{h}_{p}
$$

defines a chart $P \rightarrow \mathcal{O}_{U}$ for a log-structure on $X$ with the given extension class $\left(h_{p}\right)_{p}$.

q.e.d.

The proposition gives a complete description of germs of log structures at any geometric point $\bar{x} \rightarrow X$ by systems of invertible functions $\left(h_{p}\right)_{p}$, where $h_{p}$ is defined only on $X \backslash \operatorname{supp}(p)$. Comparing the $h_{p}$ on $X(p):=\operatorname{cl}(X \backslash \operatorname{supp}(p))$ (where cl denotes closure) leads to a finer classification of log structures.

Definition 3.15. Two germs of log structures $\xi, \xi^{\prime} \in \mathcal{E} x t^{1}\left(\overline{\mathcal{M}}_{X}^{\mathrm{gp}}, \mathcal{O}_{X}^{\times}\right)_{\bar{x}}$ are said to be of the same type if any representatives $h_{p}, h_{p}^{\prime}$ in the description of Proposition 3.14 differ only by multiplication by $e_{p} \in$ $\mathcal{O}_{X(p), \bar{x}}^{\times}$, for any $p \in P$. Two log structures on an étale open set $U \rightarrow X$ 
have the same type if their germs at every geometric point $\bar{x} \rightarrow U$ have the same type.

Geometrically speaking the type of a germ of log structures is determined by the vanishing orders of extensions of $h_{p}$ along the irreducible components of $X(p) \cap \operatorname{supp}(p)$.

For a scheme $S$ let $S^{\dagger}$ denote $S$ equipped with log structure $\mathcal{M}_{S}=$ $\mathbb{N}_{S} \oplus \mathcal{O}_{S}^{\times}$. Then if $X$ is an algebraic space over $S$ a $\log$ smooth structure on $X$ over $S^{\dagger}$ is the choice of a log structure $\mathcal{M}_{X}$ on $X$ together with a log-smooth morphism $\left(X, \mathcal{M}_{X}\right) \rightarrow S^{\dagger}$. Isomorphisms are isomorphisms of the log structures on $X$ commuting with the morphisms to $S^{\dagger}$. Our principal interest is in the case $S=\operatorname{Spec} k$ with $k$ separably closed; then $S^{\dagger}$ is the standard log point. The general case is useful for studying locally trivial moduli of log smooth structures. In fact, a log smooth morphism $\left(X, \mathcal{M}_{X}\right) \rightarrow S^{\dagger}$ induces a locally trivial log smooth deformation of the fibre over any geometric point of $S$.

Given that $X$ is defined over $S$, a morphism from $\left(X, \mathcal{M}_{X}\right)$ to $S^{\dagger}$ is equivalent to the choice of a section $\rho$ of $\mathcal{M}_{X}$ with $\alpha(\rho)=0$. Here $\rho$ is the image of the generator of $\mathbb{N}$. Let $\bar{\rho}$ be the image of $\rho$ in $\overline{\mathcal{M}}_{X}$. Then we obtain the commutative diagram

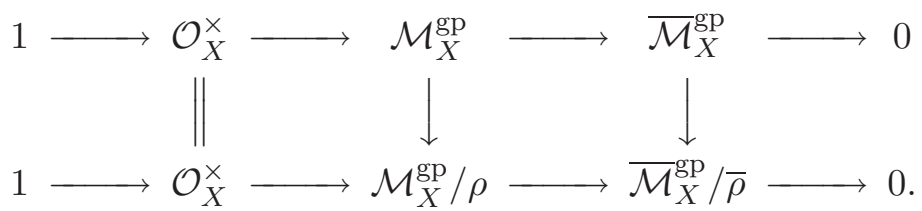

Provided that $\rho$ is nowhere nilpotent the kernel of the middle vertical arrow is isomorphic to $\mathbb{Z}$, with generator mapping to $\rho$. Hence we can tell $\rho$ from the diagram as the generator of the kernel in the middle arrow intersected with $\mathcal{M}_{X}$. Thus given a nowhere nilpotent $\bar{\rho} \in \Gamma\left(\overline{\mathcal{M}}_{X}^{\mathrm{gp}}\right)$, the set of isomorphism classes of log structures on $X$ with a morphism to $S^{\dagger}$ inducing $\bar{\rho}$ is a subset of $\operatorname{Ext}^{1}\left(\overline{\mathcal{M}}_{X}^{\mathrm{gp}} / \bar{\rho}, \mathcal{O}_{X}^{\times}\right)$. Indeed, an element of $\operatorname{Ext}^{1}\left(\overline{\mathcal{M}}_{X}^{\mathrm{gp}} / \bar{\rho}, \mathcal{O}_{X}^{\times}\right)$induces an element of $\operatorname{Ext}^{1}\left(\overline{\mathcal{M}}_{X}^{\mathrm{gp}}, \mathcal{O}_{X}^{\times}\right)$and a diagram as above by pull-back, hence a log structure and a section $\rho$ of $\mathcal{M}_{X}^{\mathrm{gp}}$. There is then a local description of the moduli of log structures with a morphism to $S^{\dagger}$ analogous to Proposition 3.14. The only difference is that we take the quotient by $\operatorname{Hom}\left(\overline{\mathcal{M}}_{X, \bar{x}}^{\mathrm{gp}} / \bar{\rho}_{\bar{x}}, \mathcal{O}_{X, \bar{x}}^{\times}\right)$rather than $\operatorname{Hom}\left(\overline{\mathcal{M}}_{X, \bar{x}}^{\mathrm{gp}}, \mathcal{O}_{X, \bar{x}}^{\times}\right)$. To impose log-smoothness we need an atlas of log smooth local models prescribing the type of log structures consistently.

Definition 3.16. Let $X$ be an algebraic space over a scheme $S$. A ghost structure on $X$ is a choice of a fine sheaf $\overline{\mathcal{M}}$ of toric monoids on $X$, a nowhere zero section $\bar{\rho} \in \Gamma(X, \overline{\mathcal{M}})$, and an étale cover of $X$, $\left\{\pi_{i}: U_{i} \rightarrow X\right\}$, with smooth morphisms

$$
U_{i} \longrightarrow S \times_{\operatorname{Spec} \mathbb{Z}} \operatorname{Spec} \mathbb{Z}\left[\overline{\mathcal{M}}_{\bar{x}_{i}}\right] /\left(z^{\bar{\rho}_{\bar{x}_{i}}}\right)
$$


for some geometric point $\bar{x}_{i} \in U_{i}$. Moreover, the log structures $U_{i}^{\dagger}$ induced by $\overline{\mathcal{M}}_{\bar{x}_{i}} \rightarrow \Gamma\left(U_{i}, \mathcal{O}_{U_{i}}\right)$ have ghost sheaves isomorphic to $\pi_{i}^{-1} \overline{\mathcal{M}}$ under an isomorphism respecting $\bar{\rho}_{\bar{x}_{i}}$, and are of the same type on overlaps of $U_{i}$ and $U_{j}$ (Definition 3.15). We usually denote a ghost structure on a scheme $X$ by $X^{g}$. If $\left(X, \mathcal{M}_{X}\right) \rightarrow S^{\dagger}$ is a log smooth structure on $X$ and $X^{g}$ is a ghost structure, we say $\left(X, \mathcal{M}_{X}\right)$ is of ghost type $X^{g}$ if there exists an isomorphism $\overline{\mathcal{M}}_{X} \rightarrow \overline{\mathcal{M}}$ identifying the given sections, and if on the open sets $U_{i}$, the $\log$ structures on $U_{i}$ induced by $\left(X, \mathcal{M}_{X}\right)$ and by $X^{g}$ are of the same type.

Example 3.17. Here is the key example of a ghost structure. Let $B$ be an integral affine manifold with singularities and toric polyhedral decomposition $\mathscr{P}$, and let $s$ be open gluing data for $\mathscr{P}$ over a scheme $S$. Then the scheme $X=X_{0}(B, \mathscr{P}, s)$ has a canonical ghost structure as follows. By the construction of $\S 2$, there exists an étale open cover of $X_{0}(B, \mathscr{P}, s)$ by sets $V(\sigma) \times S$ along with embeddings $V(\sigma) \subseteq U(\sigma)$, where $\sigma$ runs over all maximal cells. More explicitly, if $\sigma$ is a maximal cell, $P:=P_{\sigma}$ as in Definition 2.12, $\bar{\rho}:=\rho_{\sigma} \in P$ the element corresponding to the projection $M \oplus \mathbb{Z} \rightarrow \mathbb{Z}$, then

$$
\begin{aligned}
U(\sigma) & =\operatorname{Spec} \mathbb{Z}[P] \\
V(\sigma) & =\operatorname{Spec} \mathbb{Z}[P] /\left(z^{\bar{\rho}}\right)=\operatorname{Spec} \mathbb{Z}[\partial P] .
\end{aligned}
$$

Thus we obtain a canonical $\log$ structure on $V(\sigma) \times S$ from the chart $P \rightarrow \mathbb{Z}[\partial P]$, with a ghost sheaf $\overline{\mathcal{M}}_{V(\sigma) \times S}$. It is easy to see from the discussion of $\S 2$ that on overlaps, $\overline{\mathcal{M}}_{V(\sigma) \times S}$ match, gluing to give a sheaf $\overline{\mathcal{M}}_{X}$. Furthermore the sections $\bar{\rho}$ of $\overline{\mathcal{M}}_{V(\sigma) \times S}$ also glue, giving $\bar{\rho} \in \Gamma\left(X, \overline{\mathcal{M}}_{X}\right)$. Finally the identity maps $V(\sigma) \times S \rightarrow V(\sigma) \times S$ yield the smooth morphisms defining the ghost structure.

One of the fundamental problems we need to solve in this paper now arises. We wish to obtain $\log$ structures on $X_{0}(B, \mathscr{P}, s)$. The naive hope would be that the log structures induced by the above inclusions $V(\sigma) \subseteq$ $U(\sigma)$ glue to give a global $\log$ structure on $X_{0}(B, \mathscr{P}, s)$. Unfortunately, this only happens when $\Delta=\emptyset$, as we shall see.

Example 3.18. In Example 2.16, we glue together two normal crossings varieties. The $\log$ structures in fact glue, because the gluing of $V\left(\sigma_{1}\right)$ and $V\left(\sigma_{2}\right)$ along $V\left(\tau_{i}\right)$ extends to gluings of $U\left(\sigma_{1}\right)$ and $U\left(\sigma_{2}\right)$ along $U\left(\tau_{i}\right)$. However, the log smooth structures don't. In the coordinates used in that example, $\rho$ is given by the function $y z$ on $U\left(\tau_{1}\right)$ and $U\left(\tau_{2}\right)$, but $\phi(y z)=x^{-1} y z$, so the $\rho$ do not glue. (Note that $x^{-1}$ is invertible on $U\left(\tau_{i}\right)$, so the $\bar{\rho}$ 's do glue.)

In Example 2.17, even the log structures don't glue. It makes a good exercise to check this using charts. Note in this case that the gluing of $V\left(\sigma_{1}\right)$ and $V\left(\sigma_{2}\right)$ doesn't extend to a gluing of $U\left(\sigma_{1}\right)$ with $U\left(\sigma_{2}\right)$. 
As a result of this fundamental problem, in order to obtain a log structure on $X_{0}(B, \mathscr{P}, s)$, we have to understand the set of all log structures with a given ghost type. We will see that given a ghost structure $X^{g}$ there is a subsheaf $\mathcal{L S}_{X^{g}} \subset \mathcal{E} x t^{1}\left(\overline{\mathcal{M}}_{X}^{\mathrm{gp}} / \bar{\rho}, \mathcal{O}_{X}^{\times}\right)$whose sections over an étale $U \rightarrow X$ are in one-to-one correspondence with the log-smooth structures on $U$ of given ghost type.

Definition 3.19. Let $X$ be a reduced algebraic space, defined over a scheme $S$, and $X^{g}$ a ghost structure on $X$. Denote by $\mathcal{L S}_{X^{g}}$ the subsheaf of $\mathcal{E} x t^{1}\left(\overline{\mathcal{M}}_{X}^{\mathrm{gp}} / \bar{\rho}, \mathcal{O}_{X}^{\times}\right)$of germs of extension classes of the same type as prescribed by the ghost structure. We will usually drop the superscript $g$ as the ghost structure will always be clear.

Note that this definition makes sense because for sections of $\mathcal{E} x t^{1}\left(\overline{\mathcal{M}}_{X}^{\mathrm{gp}}, \mathcal{O}_{X}^{\times}\right)$to be of the same type (Definition 3.15$)$ is an open condition.

Proposition 3.20. Let $X$ be a reduced algebraic space, of finite type over a scheme $S$. Assume that $X^{g}$ is a ghost structure on $X$ with $\overline{\mathcal{M}}_{X}^{\mathrm{gp}}$ generated by $\bar{\rho}$ at the generic points. Then the set of isomorphism classes of log smooth structures on an étale set $U \rightarrow X$ of the given ghost type is canonically in bijection with $\Gamma\left(U, \mathcal{L S}_{X^{g}}\right)$.

Proof. On a reduced space any homomorphism $\varphi: \overline{\mathcal{M}}_{X}^{\mathrm{gp}} \rightarrow \mathcal{O}_{X}^{\times}$with $\varphi(\bar{\rho})=1$ is trivial, because $\bar{\rho}$ generates $\overline{\mathcal{M}}_{X}^{\mathrm{gp}}$ at the generic points. Thus $\operatorname{Hom}\left(\overline{\mathcal{M}}_{X}^{\mathrm{gp}} / \rho, \mathcal{O}_{X}^{\times}\right)=0$ and the local-global Ext spectral sequence gives

$$
\operatorname{Ext}^{1}\left(\overline{\mathcal{M}}_{X}^{\mathrm{gp}} / \bar{\rho}, \mathcal{O}_{X}^{\times}\right)=\Gamma\left(X, \mathcal{E} x t^{1}\left(\overline{\mathcal{M}}_{X}^{\mathrm{gp}} / \bar{\rho}, \mathcal{O}_{X}^{\times}\right)\right) .
$$

In view of the discussion before Definition 3.16 this shows that there is a one-to-one correspondence between isomorphism classes of log structures on an étale open set $U \rightarrow X$ together with a log morphism to $S^{\dagger}$ of the type prescribed by the ghost structure, and $\Gamma\left(U, \mathcal{L S}_{X^{g}}\right)$. It remains to show that the log morphisms arising in this way are log smooth. It suffices to check this in a neighbourhood of any geometric point $\bar{x} \rightarrow U$ and for $S=\operatorname{Spec} A$ affine. Let $\xi \in\left(\mathcal{L S}_{X^{g}}\right)_{\bar{x}}$.

The ghost structure on $U$ and Proposition 3.9 provide an étale map

$$
\phi: U \longrightarrow \operatorname{Spec} A\left[\overline{\mathcal{M}}_{X, \bar{x}} \oplus \mathbb{N}^{r}\right] /\left(z^{\bar{\rho} \bar{x}}\right)=: V
$$

with $\log$ structure associated to $\overline{\mathcal{M}}_{X, \bar{x}} \rightarrow \Gamma\left(U, \mathcal{O}_{X}\right)$ having the type prescribed by the ghost structure. For $p \in \overline{\mathcal{M}}_{X, \bar{x}}$ denote by $U(p) \subset U$ the subscheme defined by $\operatorname{Ann}\left(\phi^{*}\left(z^{p}\right)\right)$. This is the closure of $U \backslash \operatorname{supp}(p)$ when we view $p$ as a section of $\overline{\mathcal{M}}_{X}$ over $U$. We may assume that the functions from Proposition 3.14 representing $\xi$ are defined on $U(p)$ and have the form $h_{p} \phi^{*}\left(z^{p}\right)$ with $h_{p}$ invertible on $U(p)$. Define $\psi: U \rightarrow V$ by sending $(p, n) \in \overline{\mathcal{M}}_{X, \bar{x}} \oplus \mathbb{N}^{r}$ to $h_{p} \cdot \phi^{*}\left(z^{(p, n)}\right)$. The germ at $\bar{x}$ of the associated $\log$ structure equals $\xi$. It remains to show that $\psi$ is étale. 
To do this recall that étale maps between schemes are precisely those locally of the form

$$
B \longrightarrow B\left[T_{1}, \ldots, T_{n}\right] /\left(F_{1}, \ldots, F_{n}\right)
$$

with $\operatorname{det}\left(\partial F_{i} / \partial T_{j}\right)$ invertible, see for example [35], Corollary I.3.16. Clearly, to show étaleness in a neighbourhood of a geometric point $\bar{x}$, it suffices to check invertibility of the Jacobian determinant in the local ring at $\bar{x}$. Let us now write $U=\operatorname{Spec} B[\mathbf{T}] /(\mathbf{F})$ with $B=$ $A\left[\overline{\mathcal{M}}_{X, \bar{x}} \oplus \mathbb{N}^{r}\right] /\left(z^{\bar{\rho}_{\bar{x}}}\right)$ and boldface symbols denoting indexed entities. Note that the shape of the ghost sheaf on $V$ implies that $\bar{x}$ maps to the subscheme in $U$ defined by the ideal generated by $\overline{\mathcal{M}}_{X, \bar{x}} \backslash\{0\}$. We can assume without loss of generality that $\bar{x}$ maps to the subscheme in $U$ defined by the ideal generated by $\left(\overline{\mathcal{M}}_{X, \bar{x}} \oplus \mathbb{N}^{r}\right) \backslash\{0\}$. Let $p_{1}, \ldots, p_{m}$ be generators of $\partial \overline{\mathcal{M}}_{X, \bar{x}} \oplus \mathbb{N}^{r}$ as a monoid and write $X_{k}=z^{p_{k}}$. Their relation ideal is generated by finitely many polynomials $G_{\lambda}$ of the form $\prod X_{k}^{a_{k}}-\prod X_{k}^{b_{k}}$ with $\sum a_{k} p_{k}=\sum b_{k} p_{k}$ or $\prod X_{k}^{a_{k}}$ with $\sum a_{k} p_{k}=\infty$. For each $k$ choose an extension $h_{k} \in B[\mathbf{T}] /(\mathbf{F})$ of $h_{p_{k}}$ to $U$. Possibly after localizing at the $h_{k}$ we may assume $h_{k}$ to be invertible, even in $A[\mathbf{X}, \mathbf{T}] /(\mathbf{F})$. Note that localization of a $\operatorname{ring} C$ at $a$ is isomorphic to $C[T] /(a T-1)$ and hence preserves the standard description of étale maps just given.

The homomorphism defining $\psi$ is then

$$
A[\mathbf{X}] /(\mathbf{G}) \longrightarrow A[\mathbf{X}, \mathbf{T}] /(\mathbf{G}, \mathbf{F}), \quad \mathbf{X} \longmapsto \mathbf{h} \cdot \mathbf{X} .
$$

To bring this into standard form introduce new variables $\mathbf{S}$ with $\mathbf{S}=$ $\mathbf{h} \cdot \mathbf{X}$ and swap $\mathbf{X}$ and $\mathbf{S}$. Now the $h_{k}$ are invertible in $A[\mathbf{X}, \mathbf{T}] /(\mathbf{F})$, and $G_{\lambda}(\mathbf{h} \cdot \mathbf{X})=\prod h_{k}^{a_{k}} G_{\lambda}(\mathbf{X})$. Hence the ideals in $A[\mathbf{X}, \mathbf{T}] /(\mathbf{F})$ generated by $\mathbf{G}(\mathbf{X})$ and by $\mathbf{G}(\mathbf{h} \cdot \mathbf{X})$ coincide. This shows that the introduction of $\mathbf{S}$ turns the above homomorphism into the canonical inclusion

$$
A[\mathbf{X}] /(\mathbf{G}) \longrightarrow\left(A[\mathbf{X}] /(\mathbf{G}(\mathbf{X}))[\mathbf{S}, \mathbf{T}] /\left(\mathbf{X}-\mathbf{h}^{\prime} \cdot \mathbf{S}, \mathbf{F}^{\prime}\right),\right.
$$

where $\mathbf{h}^{\prime}=\mathbf{h}(\mathbf{S}, \mathbf{T}), \mathbf{F}^{\prime}=\mathbf{F}(\mathbf{S}, \mathbf{T})$. The Jacobian matrix of the relations is

$$
\left(\begin{array}{cc}
\left(-\mathbf{h}^{\prime}-\partial \mathbf{h}^{\prime} / \partial \mathbf{S} \cdot \mathbf{S}\right) & -\left(\partial \mathbf{h}^{\prime} / \partial \mathbf{T} \cdot \mathbf{S}\right) \\
\left(\partial \mathbf{F}^{\prime} / \partial \mathbf{S}\right) & \left(\partial \mathbf{F}^{\prime} / \partial \mathbf{T}\right)
\end{array}\right) .
$$

To show that the determinant of this matrix is invertible at $\bar{x}$ it suffices to show invertibility modulo $\mathbf{S}$, that is in

$$
A[\mathbf{X}, \mathbf{S}, \mathbf{T}] /\left(\mathbf{G}, \mathbf{X}-\mathbf{h}^{\prime} \cdot \mathbf{S}, \mathbf{F}^{\prime}, \mathbf{S}\right) \cong A[\mathbf{T}] /(\mathbf{F}(\mathbf{0}, \mathbf{T})) .
$$

The result is

$$
\begin{array}{rc}
\operatorname{det}\left(\begin{array}{cc}
-\operatorname{diag}(\mathbf{h}(\mathbf{0}, \mathbf{T})) & 0 \\
(\partial \mathbf{F} / \partial \mathbf{S}(\mathbf{0}, \mathbf{T})) & (\partial \mathbf{F} / \partial \mathbf{T}(\mathbf{0}, \mathbf{T}))
\end{array}\right) \\
=(-1)^{m}\left(\prod_{k=1}^{m} h_{k}(\mathbf{0}, \mathbf{T})\right) \operatorname{det}(\partial \mathbf{F} / \partial \mathbf{T})(\mathbf{0}, \mathbf{T}) .
\end{array}
$$


The right-hand side is invertible because all $h_{k}$ and $\operatorname{det}(\partial \mathbf{F} / \partial \mathbf{T})$ are invertible in $B[\mathbf{T}] /(\mathbf{F})=A[\mathbf{X}, \mathbf{T}] /(\mathbf{G}, \mathbf{F})$. Because the $X_{k} \in \operatorname{ker}\left(\mathcal{O}_{U, \bar{x}} \rightarrow\right.$ $k(\bar{x}))$ this shows that the Jacobian determinant is invertible at $\bar{x}$. Hence $\psi$ is étale in a neighbourhood of $\bar{x}$.

q.e.d.

3.3. Log structures for the fan picture. Our main goal now is to compute the sheaf $\mathcal{L S}_{X^{g}}$ for $X=X_{0}(B, \mathscr{P}, s)$ with ghost structure given by Example 3.17, and to understand global sections of $\mathcal{L S}_{X_{0}(B, \mathscr{P}, s)}$. We will in fact find that in general, there are no global sections if $\Delta \neq \emptyset$. However, we can find sections over nice open subsets of $X$, which will then define a log structure on these open subsets, which shall prove to be enough for our purposes. We will always work over $S=\operatorname{Spec} k$, where $k$ is an algebraically closed field, so we write $V(\sigma)$ instead of $V(\sigma) \times_{\mathbb{Z}}$ Spec $k$, etc.

We begin by calculating the sheaf $\mathcal{L S}_{X}$ on our standard étale covering of $X$, as follows. Let $\sigma \subseteq M_{\mathbb{R}}$ be a polytope with $\operatorname{dim} \sigma=\operatorname{dim} M_{\mathbb{R}}$, and let $P:=P_{\sigma}=C(\sigma)^{\vee} \cap(N \oplus \mathbb{Z})$ as usual, with $U(\sigma)=\operatorname{Spec} k[P]$ and $V(\sigma)=$ Spec $k[\partial P]$, also as usual. Let $\check{\Sigma}$ be the normal fan of $\sigma$ in $N_{\mathbb{R}}$. Choose some non-negative integer $r$, and set

$$
V=V(\sigma) \times \mathbb{G}_{m}^{r}=\operatorname{Spec} k\left[\partial P \oplus \mathbb{Z}^{r}\right] .
$$

$V$ carries a canonical log structure via the smooth projection map $V \rightarrow$ $V(\sigma)$, or equivalently via the obvious chart

$$
P \longrightarrow k\left[\partial P \oplus \mathbb{Z}^{r}\right] \text {. }
$$

This induces a ghost structure on $V$, and we will calculate $\mathcal{L S}_{V}$, the sheaf of log-smooth structures on $V$ with this ghost structure.

As in Definition 3.15 write $V(p)=\operatorname{cl}(V \backslash \operatorname{supp}(p))$. Let $\mathcal{F}$ be the sheaf on $V$ given by

$$
\begin{gathered}
U \longmapsto\left\{\left(h_{p}\right)_{p \in P} \mid h_{p} \text { is an invertible function on } U \cap V(p)\right. \\
\text { and } \left.h_{p} \cdot h_{q}=h_{p+q} \text { on } U \cap V(p+q)\right\} .
\end{gathered}
$$

Then $\mathcal{F}$ can be viewed as a subsheaf of $\mathcal{H o m}\left(\mathcal{R}, \mathcal{O}_{V}^{\times}\right)$via the map

$$
\Gamma(U, \mathcal{F}) \ni\left(h_{p}\right)_{p \in P} \longmapsto\left(z^{p} h_{p}\right)_{p \in P} \in \Gamma\left(U, \mathcal{H o m}\left(\mathcal{R}, \mathcal{O}_{V}^{\times}\right)\right) .
$$

Then it is clear from the definition that $\mathcal{L S}_{V}$ is the quotient

$$
\mathcal{F} / \mathcal{H o m}\left(P^{\mathrm{gp}} / \rho, \mathcal{O}_{V}^{\times}\right)=\mathcal{F} / \mathcal{H o m}\left(N, \mathcal{O}_{V}^{\times}\right) .
$$

However, it is also clear that $\mathcal{F}$ is the same as the sheaf given by

$$
\begin{gathered}
U \longmapsto\left\{\left(h_{p}\right)_{p \in \partial P \backslash\{\infty\}} \mid h_{p} \text { is an invertible function on } U \cap V(p)\right. \\
\text { and } \left.h_{p} \cdot h_{q}=h_{p+q} \text { on } U \cap V(p+q)\right\} .
\end{gathered}
$$

Note that each face $\tau$ of $\sigma$ gives a cone $\check{\tau} \in \check{\Sigma}$ and thence an irreducible closed stratum $V_{\tau}=\operatorname{Spec} k\left[(\check{\tau} \cap N) \oplus \mathbb{Z}^{r}\right]$. The scheme $V$ has irreducible components $\left\{V_{v} \mid v\right.$ is a vertex of $\left.\sigma\right\}$, and these are glued together along 
lower dimensional strata. As usual, $\tau \mapsto V_{\tau}$ is an order reversing correspondence.

For this local case we introduce shorthand notation: for a face $\tau$ of $\sigma$, we denote by $\tau^{\|}$the intersection of the lattice $M$ with the tangent space of $\tau$ at any interior point of $\tau$; this is a sublattice of $M$. As in $\S 1.5$, we can choose primitive generators $d_{\omega}$ of $\omega^{l l}$ for each edge $\omega$ of $\sigma$, and this induces a choice of vertices $v_{\omega}^{+}$and $v_{\omega}^{-}$. This induces orientations on each edge of $\sigma$.

The following definition is as in [1]. (This is no accident, see Example 4.28.)

Definition 3.21. For each 2 -face $\tau \subseteq \sigma$, we define its sign vector

$$
\epsilon_{\tau}:\{\text { dimension one faces of } \sigma\} \longrightarrow\{-1,0,+1\}
$$

by

$$
\epsilon_{\tau}(\omega)= \begin{cases}0 & \text { if } \omega \nsubseteq \tau \\ \pm 1 & \text { if } \omega \subseteq \tau\end{cases}
$$

where the sign is chosen so that if $\omega_{1}, \ldots, \omega_{N}$ are the edges of $\tau$, then the singular 1-cycle $\sum \epsilon_{\tau_{i}}\left(\omega_{i}\right) \omega_{i}$ is the oriented boundary of $\tau$ with respect to some orientation on $\tau$ and the given chosen orientations on $\omega_{i}$. This is well-defined up to sign, and we make an arbitrary choice.

Theorem 3.22. With the choices in Definition $3.21, \mathcal{L S}_{V}$ is isomorphic to the subsheaf of $\bigoplus_{\operatorname{dim} \omega=1} \mathcal{O}_{V_{\omega}}^{\times}$defined as follows. If $U \subseteq V$ is any open set, then $\Gamma\left(U, \mathcal{L} \mathcal{S}_{V}\right)$ consists of $\left(f_{\omega}\right) \in \Gamma\left(U, \bigoplus_{\operatorname{dim} \omega=1} \mathcal{O}_{V_{\omega}}^{\times}\right)$ such that for every two-dimensional face $\tau$ of $\sigma$, we have

$$
\left.\prod_{\operatorname{dim} \omega=1} d_{\omega} \otimes f_{\omega}^{\epsilon_{\tau}(\omega)}\right|_{V_{\tau}}=1 \in M \otimes_{\mathbb{Z}} \Gamma\left(U, \mathcal{O}_{V_{\tau}}^{\times}\right) .
$$

Here the product is taken in the group $M \otimes_{\mathbb{Z}} \Gamma\left(U, \mathcal{O}_{V_{\tau}}^{\times}\right)$, where $M$ is written additively and $\Gamma\left(U, \mathcal{O}_{V_{\tau}}^{\times}\right)$multiplicatively.

Proof. $\quad \mathcal{L S}_{V}$ can be viewed as a sheaf in either the Zariski topology or the étale topology. Here we will show that we get an isomorphism in the Zariski topology, though the same proof works in the étale topology. For the purpose of this proof we therefore work in the Zariski topology. (See [37], §2.1.1.)

We begin by defining a homomorphism

$$
\xi: \mathcal{F} \longrightarrow \bigoplus_{\operatorname{dim} \omega=1} \mathcal{O}_{V_{\omega}}^{\times}
$$

and then show the kernel of $\xi$ is $\mathcal{H o m}\left(N, \mathcal{O}_{V}^{\times}\right)$, and the image is the subsheaf defined by the condition (3.2).

To define $\xi$, let $h=\left(h_{p}\right)_{p \in \partial P \backslash\{\infty\}}$ be a section of $\mathcal{F}$ over an open set $U$ of $V$. For each vertex $v$ of $\sigma$, consider $p \in \check{v} \cap N$. Because $z^{p} \in k[\partial P]$ does not vanish generically on the irreducible component $V_{v}$ of $V$, the 
generic point of $V_{v}$ is not contained in $\operatorname{supp}(p)$, so $V_{v} \subseteq V(p)$. Thus in particular

$$
\left.p \longmapsto h_{p}\right|_{V_{v}} \in \Gamma\left(U, \mathcal{O}_{V_{v}}^{\times}\right)
$$

gives a multiplicative map

$$
g_{v}: \check{v} \cap N \longrightarrow \Gamma\left(U, \mathcal{O}_{V_{v}}^{\times}\right)
$$

which extends multiplicatively to a map

$$
g_{v}: N \longrightarrow \Gamma\left(U, \mathcal{O}_{V_{v}}^{\times}\right),
$$

i.e., an element $g_{v} \in M \otimes \Gamma\left(U, \mathcal{O}_{V_{v}}^{\times}\right)$. Now if $\omega$ is an edge of $\sigma$, then $\check{\omega}=\check{v}_{\omega}^{+} \cap \check{v}_{\omega}^{-}$, and for $p \in \check{\omega},\left.g_{v_{\omega}^{+}}(p)\right|_{V_{\omega}}=\left.g_{v_{\omega}^{-}}(p)\right|_{V_{\omega}}$. Thus

$$
\frac{\left.g_{v_{\omega}^{-}}\right|_{V_{\omega}}}{\left.g_{v_{\omega}^{+}}\right|_{V_{\omega}}} \in \omega^{\|} \otimes \Gamma\left(U, \mathcal{O}_{V_{\omega}}^{\times}\right),
$$

and we can write

$$
\frac{\left.g_{v_{\omega}^{-}}\right|_{V_{\omega}}}{\left.g_{v_{\omega}^{+}}\right|_{V_{\omega}}}=d_{\omega} \otimes \xi_{\omega}(h)
$$

for some $\xi_{\omega}(h)$. Then we define

$$
\xi(h)=\left(\xi_{\omega}(h)\right)_{\operatorname{dim} \omega=1} \in \Gamma\left(U, \underset{\operatorname{dim} \omega=1}{\bigoplus} \mathcal{O}_{V_{\omega}}^{\times}\right) .
$$

Now by the construction of $\xi$, the kernel of $\xi$ clearly contains $\mathcal{H o m}(N$, $\left.\mathcal{O}_{V}^{\times}\right)$. On the other hand, suppose $h \in \operatorname{ker} \xi$ on an open set $U$. We wish to show $h$ is induced by an element of $\operatorname{Hom}\left(N, \mathcal{O}_{U}^{\times}\right)$, i.e., there is an $h^{\prime} \in \mathcal{H o m}\left(N, \mathcal{O}_{U}^{\times}\right)$such that $\left.h^{\prime}(p)\right|_{V(p)}=h_{p}$. To construct such an $h^{\prime}$, let $x \in U$ and let $\tau$ be the largest face of $\sigma$ such that $x \in V_{\tau}$. Let $v_{1}, \ldots, v_{r}$ be the vertices of $\tau$, so that $x \in \bigcap_{i=1}^{r} V_{v_{i}}$. Then for $p \in N$, $g_{v_{i}}(p) \in \Gamma\left(U, \mathcal{O}_{V_{v_{i}}}^{\times}\right)$, where $g_{v_{i}} \in M \otimes \Gamma\left(U, \mathcal{O}_{V_{v_{i}}}^{\times}\right)$is as above. If $\omega$ is an edge of $\tau$, say with vertices $v_{i}$ and $v_{j}$, then $\left.g_{v_{i}}(p)\right|_{V_{\omega}}=\left.g_{v_{j}}(p)\right|_{V_{\omega}}$, since $h \in \operatorname{ker} \xi$. Thus the functions $\left.g_{v_{i}}(p)\right|_{X_{v_{i}}}$ glue to give a function $h^{\prime}(p)$ in a neighbourhood of $x$. Doing this for an open covering of $U$ gives a function $h^{\prime}(p)$ defined everywhere on $U$. Since each $g_{v}$ is multiplicative, so is $h^{\prime}$, so that $h^{\prime} \in \mathcal{H} \operatorname{Hom}\left(N, \mathcal{O}_{V}^{\times}\right)$. Clearly by construction, $\left.h^{\prime}(p)\right|_{V(p)}=$ $h_{p}$. Thus $\operatorname{ker} \xi=\mathcal{H o m}\left(N, \mathcal{O}_{V}^{\times}\right)$.

We next consider the image of $\xi$. If $\tau$ is any 2-face of $\sigma$, with vertices $v_{1}, \ldots, v_{N}$ in cyclic order, $\omega_{i}$ the edge connecting $v_{i}$ with $v_{i+1}$ (indices modulo $N)$ and $h \in \Gamma(U, \mathcal{F})$, then

$$
\left.\prod_{i=1}^{N} d_{\omega_{i}} \otimes \xi_{\omega_{i}}(h)^{\epsilon_{\tau}\left(\omega_{i}\right)}\right|_{V_{\tau}}=\frac{\left.g_{v_{1}}\right|_{V_{\tau}}}{\left.g_{v_{2}}\right|_{V_{\tau}}} \cdot \frac{\left.g_{v_{2}}\right|_{V_{\tau}}}{\left.g_{v_{3}}\right|_{V_{\tau}}} \cdots \frac{\left.g_{v_{N}}\right|_{V_{\tau}}}{\left.g_{v_{1}}\right|_{V_{\tau}}}=1 .
$$

Thus $\xi(h)$ satisfies condition (3.2). 
Conversely, we need to show that $\xi$ surjects onto the subsheaf defined by (3.2). We will show surjectivity on stalks, so we fix a point $x \in V$. Suppose we are given an element

$$
f=\left(f_{\omega}\right) \in \bigoplus_{\operatorname{dim} \omega=1} \mathcal{O}_{V_{\omega}, x}^{\times}
$$

satisfying (3.2). For each $\omega \subseteq \sigma$, let $M_{\omega}=M \otimes \mathcal{O}_{V_{\omega}, x}^{\times}$, and whenever $\omega_{1} \subseteq \omega_{2}$, let $\varphi_{\omega_{2} \omega_{1}}: M_{\omega_{1}} \rightarrow M_{\omega_{2}}$ be given by restriction of functions. Then $\left\{M_{\omega}\right\}_{\omega \subseteq \sigma}$ forms a system as in $\S$ A.1. Thus we obtain a complex $C_{\text {phd }}^{\bullet}$ as in $\S \bar{A} .2$. Furthermore, $k:=\left(k_{\omega}\right)=\left(d_{\omega} \otimes f_{\omega}\right)_{\substack{\omega \subseteq \sigma \\ \text { dim }}}$ is an element of $C_{\mathrm{phd}}^{1}$ with $d_{\mathrm{phd}}(\alpha)=0$, because of condition (3.2). However, the system $\left\{M_{\omega}\right\}$ satisfies Condition ( $*$ ) of $\S$ A.1 in exactly the same way as argued in Example A.3: giving a compatible collection on a subset of the set of faces of $\sigma$ means giving elements of various $M \otimes \mathcal{O}_{V_{\omega}, \bar{x}}^{\times}$which agree on intersections of strata. These elements therefore glue, and can be lifted to an element of $M \otimes \mathcal{O}_{V(\sigma), \bar{x}}^{\times}$. Since Condition $(*)$ holds, there exists an element $g:=\left(g_{v}\right)_{v \in \sigma} \in C_{\text {phd }}^{0}$ such that $d_{\text {phd }}(g)=k$.

We can now construct $h=\left(h_{p}\right)$ as follows. If $p \in \partial P \backslash\{\infty\}$, then $V(p)=\bigcup_{\check{v} \ni p} V_{v}$. We want to define $h_{p}$ so that $\left.h_{p}\right|_{V_{v}}=g_{v}(p)$ whenever $p \in \check{v}$. Thus we need to be able to glue the $g_{v}(p)$ for all $v$ with $p \in \check{v}$. So if $p \in \check{\omega} \subset \check{v}$, we need to know $\left.g_{v}(p)\right|_{V_{\omega}}$ is independent of $v \in \omega$. To check this, it is enough to check that this is true when $\omega$ is dimension one, with two vertices $v_{\omega}^{ \pm}$. But

$$
\frac{\left.g_{v_{\omega}^{-}}(p)\right|_{V_{\omega}}}{\left.g_{v_{\omega}^{+}}(p)\right|_{V_{\omega}}}=k_{\omega}(p)=d_{\omega}(p) \otimes f_{\omega} .
$$

But as $p \in \check{\omega}, d_{\omega}(p)=0$, so this is 1 and we have the desired independence. Thus $g_{v_{\omega}^{+}}(p)$ and $g_{v_{\omega}^{-}}(p)$ glue. Thus we obtain $h_{p}$.

Finally, we need to check that $\xi(h)=f$. But if $\omega \subset \sigma$ is an edge, then

$$
d_{\omega} \otimes \xi_{\omega}(h)=\frac{\left.g_{v_{\omega}^{-}}\right|_{V_{\omega}}}{\left.g_{v_{\omega}^{+}}\right|_{V_{\omega}}}=k_{\omega}=d_{\omega} \otimes f_{\omega}
$$

by definition of $k_{\omega}$ and $\xi$. This gives the desired result, and surjectivity is proved.

q.e.d.

Example 3.23.

1) Let $\sigma \subseteq M_{\mathbb{R}}=\mathbb{R}^{n}$ be the standard simplex, i.e., the convex hull of $e_{0}=(0, \ldots, 0), e_{1}=(1,0, \ldots, 0), \ldots, e_{n}=(0, \ldots, 0,1)$. Then $V:=V(\sigma)$ can be identified with the scheme defined by $\prod z_{i}=0$ in Spec $k\left[z_{0}, \ldots, z_{n}\right]$. Let $\left(f_{\omega}\right)$ be as in the above theorem, so that $f_{\omega}$ is an invertible function on $V_{\omega}$ for $\omega \subseteq \sigma$ of dimension one. We need to impose the condition that it be in $\mathcal{L S}_{V}$. Without loss of generality, we can take the two-face $\tau$ spanned by $e_{0}, e_{1}$ and $e_{2}$. We get the condition 
that

$$
\left(f_{e_{0} e_{1}} f_{e_{1} e_{2}}^{-1}, f_{e_{1} e_{2}} f_{e_{2} e_{0}}^{-1}, 1, \ldots, 1\right)=(1, \ldots, 1)
$$

on $V_{\tau}$. But this implies that the three functions $f_{e_{0} e_{1}}, f_{e_{0} e_{2}}$ and $f_{e_{1} e_{2}}$ agree on $V_{\tau}$. One can then easily see, in fact, $\mathcal{L} \mathcal{S}_{V} \cong \mathcal{O}_{D}^{\times}$, where $D$ is the singular locus of $V$.

2) We next look at the quadruple point, i.e., $\sigma \subseteq \mathbb{R}^{2}$ with vertices $(0,0),(1,0),(1,1)$ and $(0,1)$. Starting at $(0,0)$ and proceeding counterclockwise, we have edges $\omega_{1}, \ldots, \omega_{4}$, which we orient positively, with generators $d_{i}$ of $\omega_{i}^{\|}$being $d_{1}=(1,0), d_{2}=(0,1), d_{3}=(-1,0)$, and $d_{4}=(0,-1)$. Then a choice of $f_{i} \in \mathcal{O}_{V_{\omega_{i}}}^{\times}$must satisfy $\left(f_{1} f_{3}^{-1}, f_{2} f_{4}^{-1}\right)=$ $(1,1)$ on $V_{\sigma}$, i.e., $f_{1}=f_{3}$ and $f_{2}=f_{4}$ at the point $V_{\sigma}$. Note this gives the description of $\mathcal{L S}_{V}$ as $\mathcal{O}_{D_{1}}^{\times} \times \mathcal{O}_{D_{2}}^{\times}$, where $D_{1}=V_{\omega_{1}} \cup V_{\omega_{3}}$ and $D_{2}=V_{\omega_{2}} \cup V_{\omega_{4}}$.

We now need to understand the global nature of the sheaf of log smooth structures. Given an isomorphism $f: X^{g} \rightarrow Y^{g}$ of schemes with ghost structures, there is of course a natural isomorphism

$$
f^{-1}: \mathcal{L S}_{Y} \longrightarrow f_{*} \mathcal{L S}_{X}
$$

defined as follows. For $U \subseteq X$, a section $\alpha \in \Gamma\left(f(U), \mathcal{L} \mathcal{S}_{Y}\right)$ defines a log smooth structure $f(U)^{\dagger} \rightarrow$ Spec $k^{\dagger}$. Pulling back this log structure to $U$, we get a composed map $U^{\dagger} \rightarrow f(U)^{\dagger} \rightarrow$ Spec $k^{\dagger}$ giving a log smooth structure on $U$, hence a section $f^{-1}(\alpha) \in \Gamma\left(U, \mathcal{L S}_{X}\right)$. More generally, if $f: X^{g} \rightarrow Y^{g}$ is any étale morphism such that the pull-back of any log structure on $Y$ of ghost type $Y^{g}$ to $X$ is of ghost type $X^{g}$, we similarly get a map $f^{-1}: \mathcal{L S}_{Y} \rightarrow f_{*} \mathcal{L} \mathcal{S}_{X}$.

We wish to describe $f^{-1}$ in terms of the representation of Theorem 3.22. Let $B$ be an integral affine manifold with singularities, $\mathscr{P}$ a toric polyhedral decomposition, $k$ an algebraically closed field, $S=\operatorname{Spec} k$. Let $s \in Z^{1}\left(\mathscr{P}, \mathcal{Q}_{\mathscr{P}} \otimes \mathbb{G}_{m}(S)\right)$ be open gluing data. We first return to the setup of Construction 2.15, with two maximal cells $\sigma_{1}, \sigma_{2} \in \mathscr{P}$ and $\tau=\sigma_{1} \cap \sigma_{2}$, and use all the notation of that construction. Thus we have open sets $V\left(\tau_{i}\right) \subseteq V\left(\sigma_{i}\right)$ and an isomorphism

$$
\Phi_{\sigma_{1} \sigma_{2}}: V\left(\tau_{2}\right) \longrightarrow V\left(\tau_{1}\right) .
$$

In addition, we twist this isomorphism with automorphisms

$$
s_{i}: V\left(\tau_{i}\right) \longrightarrow V\left(\tau_{i}\right),
$$

where $s_{i}$ is induced by piecewise multiplicative maps $s_{i}: \partial Q_{i} \backslash\{\infty\} \rightarrow$ $\mathbb{G}_{m}(k), Q_{i}$ as in Construction 2.15, and we have

$$
\Phi_{\sigma_{1} \sigma_{2}}(s)=s_{1}^{-1} \circ \Phi_{\sigma_{1} \sigma_{2}} \circ s_{2} .
$$

We wish to describe $\Phi_{\sigma_{1} \sigma_{2}}(s)^{-1}: \Phi_{\sigma_{1} \sigma_{2}}(s)^{*} \mathcal{L} \mathcal{S}_{V\left(\tau_{1}\right)} \rightarrow \mathcal{L S}_{V\left(\tau_{2}\right)}$.

To state our description of this map, we introduce some notation, which will be generalised in Definition 3.25 below. Assume we have 
chosen generators $d_{\omega}$ of $\Lambda_{\omega}$ for each 1-dimensional cell $\omega$ of $\mathscr{P}$, as in $\S 1.5$.

For every vertex $v$ of $\tau, \check{v}$ is a top-dimensional cone in $\check{\tau}_{i}^{-1} \check{\Sigma}_{i}$, and $\left.s_{i}\right|_{\check{v} \cap N_{i}}: \check{v} \cap N_{i} \rightarrow \mathbb{G}_{m}(k)$ extends multiplicatively to a function

$$
s_{i}^{v}: N_{i} \longrightarrow \mathbb{G}_{m}(k) .
$$

If $\omega \subseteq \tau$ is a one-dimensional cell, then $s_{i}^{v_{\omega}^{+}}$and $s_{i}^{v_{\omega}^{-}}$agree on $\check{\omega}$, so that $s_{i}^{\omega}:=s_{i}^{v_{\omega}^{-}} / s_{i}^{v_{\omega}^{+}}$can be viewed as an element of $\omega^{\|} \otimes \mathbb{G}_{m}(k)$, so in particular we can write

$$
s_{i}^{\omega}=d_{\omega} \otimes \mathrm{D}\left(s_{i}, \omega\right)
$$

for some $\mathrm{D}\left(s_{i}, \omega\right) \in \mathbb{G}_{m}(k)$. Finally, a cell $\omega \subseteq \tau$ induces closed strata $V_{\omega}^{i}$ of $V\left(\sigma_{i}\right)$, with $V_{\omega}^{i}=\operatorname{Spec} k\left[\check{\omega} \cap N_{i}\right]$.

Then we have the following key calculation:

Theorem 3.24. Let

$$
f^{1}=\left(f_{\omega}^{1}\right)_{\substack{\operatorname{dim} \omega=1 \\ \omega=1}} \in \Gamma\left(U, \mathcal{L} \mathcal{S}_{V\left(\sigma_{1}\right)}\right) \subseteq \Gamma\left(U, \bigoplus_{\substack{\omega \subseteq \tau \\ \operatorname{dim} \omega=1}} \mathcal{O}_{V_{\omega}^{1}}^{\times}\right)
$$

for $U \subseteq V\left(\tau_{1}\right)$. Let $f^{2}=\left(f_{\omega}^{2}\right)=\Phi_{\sigma_{1} \sigma_{2}}(s)^{-1}\left(f^{1}\right)$. Then for each $\omega \subseteq \tau$ of dimension one,

$$
\Phi_{\sigma_{1} \sigma_{2}}(s)^{*} f_{\omega}^{1}=z^{n_{\omega}} \frac{\mathrm{D}\left(s_{1}, \omega\right)}{\mathrm{D}\left(s_{2}, \omega\right)} s_{2}\left(n_{\omega}\right) f_{\omega}^{2},
$$

where $\Phi_{\sigma_{1} \sigma_{2}}(s)^{*}$ is just the ordinary pull-back of functions and $n_{\omega}:=$ $n_{\omega}^{\sigma_{1} \sigma_{2}}$ is as defined in $\S 1.5$.

Proof. As in the proof of Theorem 3.22, we work in the Zariski topology. Let $M^{\prime} \subseteq M_{i}$ be the parallel transport of $\Lambda_{\tau}$ into $M_{i} \cong \Lambda_{y}$ for $y \in \operatorname{Int}\left(\sigma_{i}\right)$. Because this space is left invariant under monodromy, it is a canonically determined subspace of $M_{i}$. Because this subspace is canonically identified under any of the parallel transports $\psi_{v}$ considered in Construction 2.15, we don't distinguish between $M^{\prime} \subseteq M_{1}$ or $M^{\prime} \subseteq$ $M_{2}$. We then think of the cone $C^{\prime}(\tau)=\mathbb{R}_{\geq 0}(\tau \times\{1\}) \subseteq M_{\mathbb{R}}^{\prime} \oplus \mathbb{R}$ and $C^{\prime}(\tau)^{\vee} \subseteq N_{\mathbb{R}}^{\prime} \oplus \mathbb{R}$, where $N^{\prime} \cong N_{i} /\left(M^{\prime}\right)^{\perp}$, again independent of $i$.

Put $Q^{\prime}=C^{\prime}(\tau)^{\vee} \cap\left(N^{\prime} \oplus \mathbb{Z}\right), \partial Q^{\prime}$ as usual, which is determined by the fan $\check{\Sigma}_{i}\left(\check{\tau}_{i}\right)$ on $N^{\prime} \otimes \mathbb{R}$ (again independent of $i$ ). Finally, we can choose splittings of $N_{i}$ given by $\beta_{i}$ or $\delta_{i}$

$$
0 \longrightarrow\left(M^{\prime}\right)^{\perp} \stackrel{\gamma_{i}}{\longleftrightarrow} N_{i} \stackrel{\epsilon_{i}}{\stackrel{\delta_{i}}{\longleftarrow}} N^{\prime} \longrightarrow 0 .
$$

This splitting is arbitrary, but the choice will prove irrelevant. Note that $\left(M^{\prime}\right)^{\perp}$ is canonically a subspace of $N_{i}$ isomorphic to $\mathbb{Z}^{d}$. In the following we therefore sometimes omit $\gamma_{i}$ from the notation. The splittings induce 
isomorphisms

$$
\begin{aligned}
Q_{i} & =\mathbb{Z}^{d} \oplus Q^{\prime} \\
\partial Q_{i} & =\mathbb{Z}^{d} \oplus \partial Q^{\prime} .
\end{aligned}
$$

We will use $\beta_{i}, \gamma_{i}, \delta_{i}, \epsilon_{i}$ for the maps induced by these splittings also. We also write $\epsilon_{i}$ for the composition

$$
P_{i} \hookrightarrow Q_{i} \longrightarrow Q^{\prime}
$$

We use $\bar{\delta}_{i}: Q^{\prime} \rightarrow \partial Q_{i}$ for $\delta_{i}$ composed with the map

$$
Q_{i} \longrightarrow \partial Q_{i}, \quad p \longmapsto \begin{cases}p & p \in \partial Q_{i} \\ \infty & \text { otherwise }\end{cases}
$$

We will first understand how log charts behave under pull-backs. Note $V\left(\tau_{i}\right)=\operatorname{Spec} k\left[\partial Q_{i}\right]$. Suppose we are given a chart

$$
\alpha_{1}: Q^{\prime} \longrightarrow k\left[\partial Q_{1}\right]
$$

of the form

$$
\alpha_{1}(q)=h_{q} z^{\bar{\delta}_{1}(q)}
$$

where $h_{q}$ is an invertible function on $\operatorname{cl}\left\{z^{\bar{\delta}_{1}(q)} \neq 0\right\}$ and $h_{q_{1}} \cdot h_{q_{2}}=$ $h_{q_{1}+q_{2}}$ where defined. Recall the map $\Phi_{\sigma_{1} \sigma_{2}}: V\left(\tau_{2}\right) \rightarrow V\left(\tau_{1}\right)$ is induced by $\phi: \partial Q_{1} \rightarrow \partial Q_{2}$ defined in Construction 2.15. We also denote by $\phi: k\left[\partial Q_{1}\right] \rightarrow k\left[\partial Q_{2}\right]$ the induced map.

We then pull back via $\Phi_{\sigma_{1} \sigma_{2}}(s)$ the log structure by composing this chart with $\Phi_{\sigma_{1} \sigma_{2}}(s)^{*}$, i.e., set $\alpha_{2}$ to be the composition

$$
\alpha_{2}: Q^{\prime} \stackrel{\alpha_{1}}{\longrightarrow} k\left[\partial Q_{1}\right] \stackrel{\Phi_{\sigma_{1} \sigma_{2}}(s)^{*}}{\longrightarrow} k\left[\partial Q_{2}\right]
$$

Thus

$$
\begin{aligned}
& \alpha_{2}(q)=\Phi_{\sigma_{1} \sigma_{2}}(s)^{*}\left(z^{\bar{\delta}_{1}(q)} h_{q}\right) \\
& \quad=s_{1}\left(\bar{\delta}_{1}(q)\right)^{-1} s_{2}\left(\phi\left(\bar{\delta}_{1}(q)\right)\right) z^{\phi \bar{\delta}_{1}(q)} \Phi_{\sigma_{1} \sigma_{2}}(s)^{*}\left(h_{q}\right) .
\end{aligned}
$$

We can write

$$
\phi\left(\bar{\delta}_{1}(q)\right)=\gamma_{2} \beta_{2} \phi \bar{\delta}_{1}(q)+\delta_{2} \epsilon_{2} \phi \bar{\delta}_{1}(q) .
$$

By monodromy invariance of $N^{\prime}$, it follows that $\epsilon_{2} \circ \phi \circ \bar{\delta}_{1}: Q^{\prime} \rightarrow \partial Q^{\prime}$ is just the projection. Let

$$
\chi:=\beta_{2} \circ \phi \circ \bar{\delta}_{1}: Q^{\prime} \longrightarrow\left(M^{\prime}\right)^{\perp} \subseteq \partial Q_{2} .
$$

Then

$$
\alpha_{2}(q)=\left[z^{\chi(q)} \Phi_{\sigma_{1} \sigma_{2}}(s)^{*}\left(h_{q}\right) s_{1}\left(\bar{\delta}_{1}(q)\right)^{-1} s_{2}\left(\phi\left(\bar{\delta}_{1}(q)\right)\right)\right] \cdot z^{\bar{\delta}_{2}(q)} .
$$

Now we need to understand how these charts correspond to sections of $\mathcal{L S}_{V\left(\sigma_{i}\right)}$ over $V\left(\tau_{i}\right)$ using the representation given in Theorem 3.22. If we are given a chart

$$
\alpha_{i}: Q^{\prime} \longrightarrow k\left[\partial Q_{i}\right]
$$


with $\alpha_{i}(q)=h_{q}^{i} z^{\bar{\delta}_{i}(q)}$, let

$$
\alpha_{i}^{\prime}: P_{i} \longrightarrow k\left[\partial Q_{i}\right]
$$

be given by

$$
\alpha_{i}^{\prime}(p)= \begin{cases}h_{\epsilon_{i}(p)}^{i} z^{p}, & \text { if } p \text { is in a face of } P_{i} \text { containing } \check{\tau}_{i} \\ 0 & \text { otherwise. }\end{cases}
$$

It is then easy to see that the charts $\alpha_{i}^{\prime}$ and $\alpha_{i}$ induce the same log structure on $V\left(\tau_{i}\right)$. The chart $\alpha_{i}^{\prime}$ then coincides with a section $h^{i}=$ $\left(h_{\epsilon_{i}(p)}^{i}\right)_{p \in \partial Q_{i} \backslash\{\infty\}}$ of the sheaf $\mathcal{F}_{i}$ (corresponding to the sheaf $\mathcal{F}$ in the language of Theorem 3.22). For $v \in \tau$ a vertex, we let $g_{v}^{i} \in M_{i} \otimes \mathcal{O}_{V_{v}^{i}}^{\times}$be the multiplicative extension of the map $\left.\check{v} \cap N_{i} \ni p \mapsto h_{\epsilon_{i}(p)}^{i}\right|_{V_{v}^{i}} \in \mathcal{O}_{V_{v}^{i}}^{\times}$. Then the map

$$
\xi^{i}: \mathcal{F} \longrightarrow \mathcal{L S}_{V\left(\sigma_{i}\right)} \subseteq \bigoplus_{\substack{\omega \subseteq \sigma_{i} \\ \operatorname{dim} \omega=1}} \mathcal{O}_{V_{\omega}^{i}}^{\times}
$$

is given on $V_{\omega}^{i}$ by

$$
d_{\omega} \otimes \xi_{\omega}^{i}(h)=\frac{\left.g_{v_{\omega}^{-}}^{i}\right|_{V_{\omega}^{i}}}{\left.g_{v_{\omega}^{+}}^{i}\right|_{V_{\omega}^{i}}} .
$$

Now we are ready to compare the answers for $\alpha_{1}$ and $\alpha_{2}$ given by formulae (3.4) and (3.5), by applying this, for

$$
h_{q}^{1}=h_{q}
$$

and

$$
h_{q}^{2}=\left[\Phi_{\sigma_{1} \sigma_{2}}(s)^{*}\left(h_{q}\right)\right] z^{\chi(q)} s_{1}\left(\bar{\delta}_{1}(q)\right)^{-1} s_{2}\left(\phi\left(\bar{\delta}_{1}(q)\right)\right),
$$

for $q \in Q^{\prime}$. If $v$ is any vertex of $\tau$ we obtain, for $p \in \check{v} \cap N_{1}$,

$$
g_{v}^{1}(p)=h_{\epsilon_{1}(p)}^{1}
$$

and for $p \in \breve{v} \cap N_{2}$,

$$
g_{v}^{2}(p)=\left[\Phi_{\sigma_{1} \sigma_{2}}(s)^{*}\left(h_{\epsilon_{2}(p)}^{1}\right)\right] z^{\chi\left(\epsilon_{2}(p)\right)} \frac{s_{2}\left(\phi\left(\delta_{1}\left(\epsilon_{2}(p)\right)\right)\right)}{s_{1}\left(\delta_{1}\left(\epsilon_{2}(p)\right)\right)} .
$$

Here we are using the canonical identification of the quotients $N^{\prime}$ of $N_{1}$ and $N_{2}$ to make sense of $h_{\epsilon_{2}(p)}^{1}$. We wish to compare $g_{v}^{1}$ and $g_{v}^{2}$, so we will for the moment choose any vertex $w$ of $\tau$, and use the map

$$
\psi_{w}^{-1} \times \Phi_{\sigma_{1} \sigma_{2}}(s)^{*}: M_{1} \times \mathcal{O}_{V_{v}^{1} \cap V\left(\tau_{1}\right)}^{\times} \longrightarrow M_{2} \times \mathcal{O}_{V_{v}^{2} \cap V\left(\tau_{2}\right)}^{\times} .
$$


Then for $p \in \check{v} \cap N_{2}$,

$$
\begin{aligned}
{\left[\psi_{w}^{-1} \times\right.} & \left.\Phi_{\sigma_{1} \sigma_{2}}(s)^{*}\left(g_{v}^{1}\right)\right](p) / g_{v}^{2}(p) \\
& =\left(g_{v}^{2}(p)\right)^{-1}\left[\Phi_{\sigma_{1} \sigma_{2}}(s)^{*}\left(g_{v}^{1}\left(\left(\psi_{w}^{-1}\right)^{t}(p)\right)\right)\right] \\
& =\left(\left[\Phi_{\sigma_{1} \sigma_{2}}(s)^{*}\left(h_{\epsilon_{2}(p)}^{1}\right)\right]^{-1} z^{-\chi\left(\epsilon_{2}(p)\right)} \frac{s_{1}\left(\delta_{1}\left(\epsilon_{2}(p)\right)\right)}{s_{2}\left(\phi\left(\delta_{1}\left(\epsilon_{2}(p)\right)\right)\right)}\right) \\
& \cdot \Phi_{\sigma_{1} \sigma_{2}}(s)^{*}\left(h_{\epsilon_{1}\left(\left(\psi_{w}^{-1}\right)^{t}(p)\right)}^{1}\right) \\
& =z^{-\chi\left(\epsilon_{2}(p)\right)} \frac{s_{1}\left(\delta_{1}\left(\epsilon_{2}(p)\right)\right)}{s_{2}\left(\phi\left(\delta_{1}\left(\epsilon_{2}(p)\right)\right)\right)},
\end{aligned}
$$

where we used $\epsilon_{1}\left(\left(\psi_{w}^{-1}\right)^{t}(p)\right)=\epsilon_{2}(p)$. This must be extended linearly to all of $N_{2}$, which we do as follows. Recall that $\chi=\beta_{2} \circ \phi \circ \bar{\delta}_{1}$, and on $\check{v} \cap N_{1}, \phi$ is linear, given by parallel transport $\psi_{v}^{t}: N_{1} \rightarrow N_{2}$. Furthermore, on $\breve{v} \cap N_{i}, s_{i}$ extends to give the group homomorphism

$$
s_{i}^{v}: N_{i} \longrightarrow \mathbb{G}_{m}(k) .
$$

Thus for $p \in N_{2}$, we have

$$
\begin{aligned}
& \frac{\left[\psi_{w}^{-1} \times \Phi_{\sigma_{1} \sigma_{2}}(s)^{*}\left(g_{v}^{1}\right)\right](p)}{g_{v}^{2}(p)} \\
& \quad=z^{-\beta_{2} \circ \psi_{v}^{t} \circ \delta_{1} \circ \epsilon_{2}(p)} s_{1}^{v}\left(\delta_{1}\left(\epsilon_{2}(p)\right)\right) s_{2}^{v}\left(\psi_{v}^{t}\left(\delta_{1}\left(\epsilon_{2}(p)\right)\right)\right)^{-1} .
\end{aligned}
$$

We now compare $d_{\omega} \otimes \xi_{\omega}^{1}(h)$ and $d_{\omega} \otimes \xi_{\omega}^{2}(h)$ whenever $\omega$ is an edge of $\tau$. Note that $\omega^{\|} \subseteq M^{\prime}$, so we can view $\omega^{\| l}$ equally as a subspace of $M_{1}$ or $M_{2}$, but to be precise, we use parallel transport $\psi_{v_{\omega}^{-}}^{-1}: M_{1} \rightarrow M_{2}$ to make the identification; this is a convenient arbitrary choice. We then make the comparison using

$$
\psi_{v_{\omega}^{-}}^{-1} \times \Phi_{\sigma_{1} \sigma_{2}}(s)^{*}: \omega^{\|} \times \mathcal{O}_{V_{\omega}^{1}}^{\times} \longrightarrow \omega^{\|} \times \mathcal{O}_{V_{\omega}^{2}}^{\times} .
$$

Then for $p \in N_{2}$,

$$
\begin{aligned}
& \frac{\left[\left(\psi_{v_{\omega}^{-}}^{-1} \times \Phi_{\sigma_{1} \sigma_{2}}(s)^{*}\right)\left(d_{\omega} \otimes \xi_{\omega}^{1}(h)\right)\right](p)}{\left(d_{\omega} \otimes \xi_{\omega}^{2}(h)\right)(p)} \\
= & \frac{\left[\left(\psi_{v_{\omega}^{-}}^{-1} \times \Phi_{\sigma_{1} \sigma_{2}}(s)^{*}\right)\left(g_{v_{\omega}^{-}}^{1} \cdot\left(g_{v_{\omega}^{+}}^{1}\right)^{-1}\right)\right](p)}{g_{v_{\omega}^{-}}^{2}(p) \cdot\left(g_{v_{\omega}^{+}}^{2}\right)^{-1}(p)} \\
= & \frac{z^{-\beta_{2} \circ \psi_{v_{\omega}^{-}}^{t} \circ \delta_{1} \circ \epsilon_{2}(p)}}{z^{-\beta_{2} \circ \psi_{v_{\omega}^{+}}^{t} \circ \delta_{1} \circ \epsilon_{2}(p)}} \cdot \frac{s_{1}^{v_{\omega}^{-}}\left(\delta_{1}\left(\epsilon_{2}(p)\right)\right)}{s_{1}^{v_{\omega}^{+}}\left(\delta_{1}\left(\epsilon_{2}(p)\right)\right)} \cdot \frac{s_{2}^{v_{\omega}^{-}}\left(\psi_{v_{\omega}^{-}}^{t}\left(\delta_{1}\left(\epsilon_{2}(p)\right)\right)\right)^{-1}}{s_{2}^{v_{\omega}^{+}}\left(\psi_{v_{\omega}^{+}}^{t}\left(\delta_{1}\left(\epsilon_{2}(p)\right)\right)\right)^{-1}} .
\end{aligned}
$$

The first factor is

$$
z^{\beta_{2} \circ\left(\psi_{v_{\omega}^{+}}^{t}-\psi_{v_{\bar{\omega}}^{-}}^{t}\right) \circ \delta_{1} \circ \epsilon_{2}(p)},
$$


and using $\delta_{1} \circ \epsilon_{1}(p)=p-\gamma_{1} \circ \beta_{1}(p)$ for $p \in N_{1}$ and $\epsilon_{2}(p)=\epsilon_{1}\left(\left(\psi_{v_{\omega}^{-}}^{-1}\right)^{t}(p)\right)$, we see that

$$
\begin{aligned}
\beta_{2} \circ\left(\psi_{v_{\omega}^{+}}^{t}-\psi_{v_{\omega}^{-}}^{t}\right) \circ \delta_{1} \circ \epsilon_{2}(p) & =\beta_{2} \circ\left(\psi_{v_{\omega}^{+}}^{t}-\psi_{v_{\omega}^{-}}^{t}\right)\left(\left(\psi_{v_{\omega}^{-}}^{-1}\right)^{t}(p)\right) \\
& =\beta_{2} \circ\left(\psi_{v_{\omega}^{+}}^{t} \circ\left(\psi_{v_{\omega}^{-}}^{-1}\right)^{t}-I\right)(p) .
\end{aligned}
$$

In this computation a term $\gamma_{1} \circ \beta_{1}(p) \in\left(M^{\prime}\right)^{\perp}$ cancels because $\left.\psi_{v_{\omega}^{+}}^{t}\right|_{\left(M^{\prime}\right)^{\perp}}$ $=\left.\psi_{v_{\omega}^{-}}^{t}\right|_{\left(M^{\prime}\right)^{\perp}}$. In addition, the factor $\beta_{2}$ is unnecessary, as $\left(\psi_{v_{\omega}^{+}}^{t} \circ\right.$ $\left.\left(\psi_{v_{\omega}^{-}}^{-1}\right)^{t}-I\right)(p)$ is already contained in $\left(M^{\prime}\right)^{\perp}$. But in the notation of $\S 1.5,\left(T_{\omega}^{\sigma_{1} \sigma_{2}}\right)^{t}=\psi_{v_{\omega}^{+}}^{t} \circ\left(\psi_{v_{\omega}^{-}}^{t}\right)^{-1}$. Now

$$
\left(T_{\omega}^{\sigma_{1} \sigma_{2}}\right)^{t}(n)=n+\left\langle n, d_{\omega}\right\rangle n_{\omega}
$$

by the definition of $n_{\omega}$. Thus the first factor is

$$
z^{\left(\left(T_{\omega}^{\sigma_{1} \sigma_{2}}\right)^{t}-I\right)(p)}=z^{\left\langle p, d_{\omega}\right\rangle n_{\omega}} .
$$

The second factor is

$s_{1}^{\omega}\left(\delta_{1}\left(\epsilon_{1}\left(\left(\psi_{v_{\omega}^{-}}^{-1}\right)^{t}(p)\right)\right)=s_{1}^{\omega}\left(\left(\psi_{v_{\omega}^{-}}^{-1}\right)^{t}(p)-\gamma_{1} \beta_{1}\left(\psi_{v_{\omega}^{-}}^{-1}\right)^{t}(p)\right)=s_{1}^{\omega}\left(\left(\psi_{v_{\omega}^{-}}^{-1}\right)^{t}(p)\right)\right.$.

The last factor is similarly

$$
\frac{s_{2}^{v_{\omega}^{+}}\left(\psi_{v_{\omega}^{+}}^{t}\left(\psi_{v_{\omega}^{-}}^{-1}\right)^{t}(p)\right) s_{2}^{v_{\omega}^{+}}\left(\psi_{v_{\omega}^{+}}^{t} \gamma_{1} \beta_{1}\left(\psi_{v_{\omega}^{-}}^{-1}\right)^{t}(p)\right)^{-1}}{s_{2}^{v_{\omega}^{-}}(p) s_{2}^{v_{\omega}^{-}}\left(\psi_{v_{\omega}^{-}}^{t} \gamma_{1} \beta_{1}\left(\psi_{v_{\omega}^{-}}^{-1}\right)^{t}(p)\right)^{-1}} .
$$

Using

$$
s_{2}^{v_{\omega}^{+}}=s_{2}^{v_{\omega}^{-}} \text {and } \psi_{v_{\omega}^{+}}^{t}=\psi_{v_{\omega}^{-}}^{t} \text { on }\left(\omega^{\|}\right)^{\perp} \supseteq\left(M^{\prime}\right)^{\perp},
$$

the last factors cancel and this is

$$
\frac{s_{2}^{v_{\omega}^{+}}\left(\left(T_{\omega}^{\sigma_{1} \sigma_{2}}\right)^{t}(p)\right)}{s_{2}^{v_{\omega}^{-}}(p)}=\frac{s_{2}^{v_{\omega}^{+}}\left(p+\left\langle p, d_{\omega}\right\rangle n_{\omega}\right)}{s_{2}^{v_{\omega}^{-}}(p)}=s_{2}^{\omega}(p)^{-1}\left(s_{2}^{v_{\omega}^{+}}\left(n_{\omega}\right)\right)^{\left\langle p, d_{\omega}\right\rangle} .
$$

Putting this together,

$$
\begin{aligned}
& \frac{\left[\left(\psi_{v_{\omega}^{-}}^{-1} \times \Phi_{\sigma_{1} \sigma_{2}}(s)^{*}\right)\left(d_{\omega} \otimes \xi_{\omega}^{1}(h)\right)\right](p)}{\left(d_{\omega} \otimes \xi_{\omega}^{2}(h)\right)(p)} \\
& \quad=z^{\left\langle p, d_{\omega}\right\rangle n_{\omega}} s_{1}^{\omega}\left(\left(\psi_{v_{\omega}^{-}}^{-1}\right)^{t}(p)\right) s_{2}^{\omega}(p)^{-1} s_{2}^{v_{\omega}^{+}}\left(n_{\omega}\right)^{\left\langle p, d_{\omega}\right\rangle} .
\end{aligned}
$$

Thus if $f_{\omega}^{i}=\xi_{\omega}^{i}(h)$ and in view of $s_{i}^{\omega}=d_{\omega} \otimes \mathrm{D}\left(s_{i}, \omega\right)$ we see that

$$
\frac{\Phi_{\sigma_{1} \sigma_{2}}(s)^{*} f_{\omega}^{1}}{f_{\omega}^{2}}=z^{n_{\omega}} \frac{\mathrm{D}\left(s_{1}, \omega\right)}{\mathrm{D}\left(s_{2}, \omega\right)} s_{2}^{v_{\omega}^{+}}\left(n_{\omega}\right) .
$$

Note that since $n_{\omega} \in\left(M^{\prime}\right)^{\perp}, s_{2}^{v_{\omega}^{+}}\left(n_{\omega}\right)=s_{2}\left(n_{\omega}\right)$. This formula is now completely independent of the choices of splitting.

q.e.d. 
We are now ready to summarize the discussion of this section. First, we define the global versions of the data appearing in Theorem 3.24.

Definition 3.25.

1) Let $B$ be an integral affine manifold with singularities and $\mathscr{P}$ a toric polyhedral decomposition, and suppose $d_{\omega}$ has been chosen as in $\S 1.5$ for each $\omega \in \mathscr{P}, \operatorname{dim} \omega=1$. If $\tau \in \mathscr{P}, s \in P \breve{M}(\tau)$ (see Definition 2.24), $h: v \rightarrow \tau$ with $v$ a vertex, we define $s^{h} \in \Lambda_{v} \otimes \mathbb{G}_{m}(k)$ to be the germ of $s \in \Gamma\left(\tilde{\tau}, \pi^{*}\left(\mathcal{Q}_{\mathscr{P}} \otimes \mathbb{G}_{m}(k)\right)\right)$ at the vertex $v_{h}$ of $\tilde{\tau}$ corresponding to $h$.

2) If $\operatorname{dim} \tau \geq 1$, and we are given morphisms $e: \omega \rightarrow \tau, f: \tau \rightarrow \sigma$ with $\operatorname{dim} \omega=1, \sigma \in \mathscr{P}_{\max }$, giving a diagram

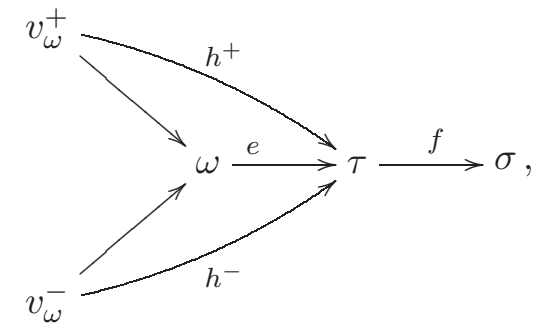

we can parallel transport $s^{h^{+}} \in \Lambda_{v_{\omega}^{+}} \otimes \mathbb{G}_{m}$ and $s^{h^{-}} \in \Lambda_{v_{\omega}^{-}} \otimes \mathbb{G}_{m}$ to $\Lambda_{y} \otimes \mathbb{G}_{m}$ with $y \in \operatorname{Int}(\sigma)$ via $f \circ h^{+}$and $f \circ h^{-}$. Then we define $\mathrm{D}(s, e, f) \in \mathbb{G}_{m}$ (or $\mathrm{D}(s, \omega, \sigma)$ when $e$ and $f$ are understood) by

$$
d_{\omega} \otimes \mathrm{D}(s, e, f)=\frac{s^{h^{-}}}{s^{h^{+}}} .
$$

One should think of $\mathrm{D}(s, e, f)$ as monodromy information for the gluing data $s$.

Remark 3.26. We emphasize the dependence of $\mathrm{D}(s, e, f)$ on $f$ (or

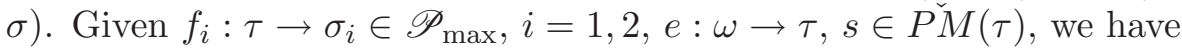
an identification $\psi: \Lambda_{y_{1}} \otimes \mathbb{G}_{m} \rightarrow \Lambda_{y_{2}} \otimes \mathbb{G}_{m}$ for $y_{i} \in \operatorname{Int}\left(\sigma_{i}\right)$ by parallel transport from $y_{1}$ to $v_{\omega}^{-}$along $f_{1} \circ h^{-}$and $v_{\omega}^{-}$to $y_{2}$ along $f_{2} \circ h^{-}$. Then under this identification, the parallel transport of $s^{h^{-}} \in \Lambda_{v_{\omega}^{-}} \otimes \mathbb{G}_{m}$ to $\Lambda_{y_{1}} \otimes \mathbb{G}_{m}$ and $\Lambda_{y_{2}} \otimes \mathbb{G}_{m}$ are identified by $\psi$. However, if $s_{i}^{h^{+}} \in \Lambda_{y_{i}} \otimes \mathbb{G}_{m}$ is the parallel transport of $s^{h^{+}} \in \Lambda_{v_{\omega}^{+}} \otimes \mathbb{G}_{m}$ to $\Lambda_{y_{i}} \otimes \mathbb{G}_{m}$ along $f_{i} \circ h^{+}$, then

$$
\psi\left(s_{1}^{h^{+}}\right)=T_{\omega}^{f_{1} \circ e, f_{2} \circ e}\left(s_{2}^{h^{+}}\right)
$$

Thus

$$
\psi\left(s_{1}^{h^{-}} / s_{1}^{h^{+}}\right)=s_{2}^{h^{-}} / T_{\omega}^{f_{1} \circ e, f_{2} \circ e}\left(s_{2}^{h^{+}}\right) .
$$


If $n \in \check{\Lambda}_{y_{2}}$ is such that $\left\langle n, d_{\omega}\right\rangle=1$, then $\left\langle\psi^{t}(n), d_{\omega}\right\rangle=1$ also, and we see that

$$
\begin{aligned}
\mathrm{D}\left(s, e, f_{1}\right) & =s_{1}^{h^{-}}\left(\psi^{t}(n)\right) / s_{1}^{h^{+}}\left(\psi^{t}(n)\right)=s_{2}^{h^{-}}(n) /\left(T_{\omega}^{f_{1} \circ e, f_{2} \circ e}\left(s_{2}^{h^{+}}\right)\right)(n) \\
& =s_{2}^{h^{-}}(n) / s_{2}^{h^{+}}\left(n+\left\langle n, d_{\omega}\right\rangle n_{\omega}^{f_{1} \circ e, f_{2} \circ e}\right) \\
& =\mathrm{D}\left(s, e, f_{2}\right) s_{2}^{h^{+}}\left(n_{\omega}^{f_{1} \circ e, f_{2} \circ e}\right)^{-1}=\mathrm{D}\left(s, e, f_{2}\right) s\left(n_{\omega}^{f_{1} \circ e, f_{2} \circ e}\right)^{-1} .
\end{aligned}
$$

The last equality follows from the fact that $n_{\omega}^{f_{1} \circ e, f_{2} \circ e} \in \Lambda_{\tau}^{\perp}$, and hence $s_{i}^{h}\left(n_{\omega}^{f_{1} \circ e, f_{2} \circ e}\right)$ is independent of $h$ and $i$.

This explains the asymmetry in the role of $s_{1}$ and $s_{2}$ in the gluing formulae (3.3) above or (3.6) below.

Now suppose we are given $(B, \mathscr{P})$ and $s$. How do we specify a section of $\mathcal{L S}_{X_{0}(B, \mathscr{P}, s)}$ over an open set $U \subseteq X_{0}(B, \mathscr{P}, s)$ ? We have for each $\sigma \in \mathscr{P}_{\max }$ the projection $p_{\sigma}: V(\sigma) \rightarrow X_{0}(B, \mathscr{P}, s)$. So a section $f \in \Gamma\left(U, \mathcal{L S}_{X_{0}(B, \mathscr{P}, s)}\right)$ pulls back to sections

$$
f_{\sigma}=p_{\sigma}^{-1}(f) \in \Gamma\left(p_{\sigma}^{-1}(U), \mathcal{L S}_{V(\sigma)}\right) .
$$

Furthermore, for any component $\mathfrak{R}_{g_{1} g_{2}}$ of $\mathfrak{R}$ (see after Definition 2.12), $g_{i}: \tau \rightarrow \sigma_{i}$, we have

$$
f_{\sigma_{2}}=\Phi_{g_{1} g_{2}}(s)^{-1}\left(f_{\sigma_{1}}\right)
$$

on $q_{2}^{-1}(U) \cap V\left(\tau_{2}\right) \subseteq V\left(\sigma_{2}\right)$.

Of course, by the sheaf gluing axiom, the converse is true:

Theorem 3.27. Let $B$ be an integral affine manifold with singularities with a toric polyhedral decomposition $\mathscr{P}$ and open gluing data $s$ over Spec $k$. Choose $d_{\omega}$ 's as in $\S 1.5$. Let $U \subseteq X_{0}(B, \mathscr{P}, s)$ be an open subset. Then to give an element $f \in \Gamma\left(U, \mathcal{L S}_{X_{0}(B, \mathscr{P}, s)}\right)$, it is enough to give sections for each $\sigma \in \mathscr{P}_{\max }$

$$
\begin{aligned}
f_{\sigma}=\left(f_{\sigma, e}\right) \underset{\substack{e: \omega \rightarrow \sigma \\
\operatorname{dim} \omega=1}}{ } \in \Gamma\left(p_{\sigma}^{-1}(U), \mathcal{L} \mathcal{S}_{V(\sigma)}\right) \\
\subseteq \bigoplus_{\substack{e: \omega \rightarrow \sigma \\
\operatorname{dim} \omega=1}} \Gamma\left(p_{\sigma}^{-1}(U), \mathcal{O}_{V_{e}}^{\times}\right)
\end{aligned}
$$

satisfying the following property: For every connected component $\mathfrak{R}_{g_{1} g_{2}}$ of $\mathfrak{R}, g_{i}: \tau \rightarrow \sigma_{i} \in \mathscr{P}_{\max }$, and for every $h: \omega \rightarrow \tau$ with $\operatorname{dim} \omega=1$ and $e_{i}=g_{i} \circ h$, we have

$$
\Phi_{g_{1} g_{2}}(s)^{*}\left(f_{\sigma_{1}, e_{1}}\right)=z^{n_{\omega}^{e_{1} e_{2}}} \frac{\mathrm{D}\left(s_{g_{1}}, h, g_{1}\right)}{\mathrm{D}\left(s_{g_{2}}, h, g_{2}\right)} s_{g_{2}}\left(n_{\omega}^{e_{1} e_{2}}\right) f_{\sigma_{2}, e_{2}}
$$

on $p_{\sigma_{2}}^{-1}(U) \cap V\left(\tau_{2}\right)$.

Proof. This is just a summary of the discussions, especially Theorem 3.24 , above.

Note that there is no need to define the sign vectors $\epsilon_{\tau}$ from Definition 3.21 globally. The reason is that these are only used to define $\mathcal{L} \mathcal{S}_{V(\sigma)}$ as a subsheaf of $\bigoplus_{e: \omega \rightarrow \sigma} \mathcal{O}_{V_{e}}^{\times}$, and this is independent of choices 
for the sign vectors. In fact, any $\epsilon_{\tau}$ is well-defined up to an overall change of sign and this does not affect the defining equation (3.2) for $\mathcal{L S}_{V(\sigma)} \subset \bigoplus_{e: \omega \rightarrow \sigma} \mathcal{O}_{V_{e}}^{\times}$

q.e.d.

It is also helpful to give a partial description of $\mathcal{L S}_{X_{0}(B, \mathscr{P}, s)}$ in terms of closed gluings.

Theorem 3.28. Let $B$ be an integral affine manifold with singularities, $\mathscr{P}$ a toric polyhedral decomposition, and $s$ open gluing data. Choose $d_{\omega}$ 's as in $\S 1.5$. Then for each $\omega \in \mathscr{P}$ of dimension one, there is an $\mathcal{O}_{X_{\omega}}^{\times}$-torsor $\mathcal{N}_{\omega}^{\times}$such that $\mathcal{L S}_{X_{0}(B, \mathscr{P}, s)}$ is a subsheaf of

$$
\bigoplus_{\substack{\omega \in \mathscr{P} \\ \operatorname{dim} \omega=1}} q_{\omega *} \mathcal{N}_{\omega}^{\times},
$$

where $q_{\omega}: X_{\omega} \rightarrow X_{0}(B, \mathscr{P}, s)$ are the maps of Lemma 2.29. Furthermore, if $\mathcal{N}_{\omega}$ is the line bundle on $X_{\omega}$ associated to $\mathcal{N}_{\omega}^{\times}$(in particular $\mathcal{N}_{\omega}^{\times}$ is the sheaf of nowhere vanishing sections of $\left.\mathcal{N}_{\omega}\right)$ then $\mathcal{N}_{\omega}$ corresponds to the piecewise linear function $\psi_{\omega}$ on the fan $\Sigma_{\omega}$ in $\mathcal{Q}_{\omega, \mathbb{R}}$, well-defined up to linear functions, defined in Remark 1.56.

Proof. As usual write $V_{e} \subseteq X_{\omega}$ for the open subset corresponding to the maximal cone $K_{e}$ of $\Sigma_{\omega}$. It is easy to see that $\mathcal{L S}_{X_{0}(B, \mathscr{P}, s)}$ is a subsheaf of $\bigoplus q_{\omega *} \mathcal{N}_{\omega}^{\times}$, where the sum runs over the one-dimensional $\omega \in \mathscr{P}$ and $\mathcal{N}_{\omega}^{\times}$is defined by the following gluing data for the open cover $\left\{V_{e} \mid e \in \coprod_{\sigma \in \mathscr{P}_{\max }} \operatorname{Hom}(\omega, \sigma)\right\}$. We glue $\mathcal{O}_{V_{e_{1}}}^{\times}$and $\mathcal{O}_{V_{e_{2}}}^{\times}$via the identification

$$
\mathcal{O}_{V_{e_{2}}}^{\times}\left|V_{e_{1}} \cap V_{e_{2}} \longrightarrow \mathcal{O}_{V_{e_{1}}}^{\times}\right|_{V_{e_{1}} \cap V_{e_{2}}}
$$

given by

$$
f \longmapsto z^{n_{\omega}^{e_{1} e_{2}}} \cdot s^{\prime} \cdot f
$$

where $s^{\prime} \in \mathbb{G}_{m}(k)$ depends on $s$. Since $X_{\omega}$ is toric, we can just as well assume $s^{\prime}=1$, i.e., take the trivial open gluing data and we will get an isomorphic $\mathcal{O}_{X_{\omega}}^{\times}$-torsor. Then the result that $\psi_{\omega}$ determines $\mathcal{N}_{\omega}$ follows from the standard recipe for obtaining a line bundle from a piecewise linear function (see [38], p. 69, keeping in mind the sign convention of Remark 0.1).

q.e.d.

Corollary 3.29. Let $B$ be an integral affine manifold with singularities, with a toric polyhedral decomposition $\mathscr{P}$. If there exists a log smooth structure on $X_{0}(B, \mathscr{P}, s)$ for some open gluing data $s$, then the integral affine structure on $B_{0} \subseteq B$ can be extended to $B$, so $B$ in fact has no singularities.

Proof. If there is a $\log$ smooth structure, then each of the torsors $\mathcal{N}_{\omega}^{\times}$has sections, hence is a trivial torsor. Thus $\psi_{\omega}$ is a linear function. By the definition of $\psi_{\omega}, n_{\omega}^{e_{1} e_{2}}$ is the difference of linear extensions of $\psi_{\omega}$ 
on different cones of $\Sigma_{\omega}$, hence $n_{\omega}^{e_{1} e_{2}}=0$ for all $e_{1}, e_{2}, \omega$, and hence by Proposition 1.27, the affine structure extends to $B$.

In general, we now see that the existence of singularities on $B$ implies there does not exist a global section of $\mathcal{L S}_{X_{0}(B, \mathscr{P}, s)}$. Therefore, to proceed, we have to allow the log smooth structure to break down. We shall study this in the next section.

Example 3.30. If $X_{0}(B, \mathscr{P}, s)$ is normal crossings (i.e., every $\sigma \in$ $\mathscr{P}_{\max }$ is a standard simplex), then it follows from Example 3.23, (1), that $\mathcal{L S}_{X_{0}(B, \mathscr{P}, s)}$ is an $\mathcal{O}_{D}^{\times}$-torsor, where $D=\operatorname{Sing}\left(X_{0}(B, \mathscr{P}, s)\right)$. One can show $[\mathbf{1 2}],[\mathbf{2 9}]$ that $\mathcal{L S}_{X_{0}(B, \mathscr{P}, s)}$ is the $\mathcal{O}_{D}^{\times}$-torsor associated to the line bundle $\mathcal{E} x t^{1}\left(\Omega_{X_{0}(B, \mathscr{P}, s)}^{1}, \mathcal{O}_{X_{0}(B, \mathscr{P}, s)}\right)$, the local $T^{1}$-sheaf. There exists a $\log$ smooth structure on $X_{0}(B, \mathscr{P}, s)$ if and only if this is the trivial line bundle. In any normal crossings case, $q_{\omega}^{*} \mathcal{L} \mathcal{S}_{X_{0}(B, \mathscr{P}, s)}$ is the $\mathcal{O}_{X_{\omega}}^{\times}$-torsor determined by Theorem 3.28 using monodromy data near $\omega$. Explicitly, in dimension two, if $\omega \in \mathscr{P}$ is an edge containing a singular

point of the affine structure with monodromy $\left(\begin{array}{ll}1 & n \\ 0 & 1\end{array}\right)$ in a suitable basis (chosen so that $n>0$ if and only if the singularity is positive in the sense of Definition 1.54) then $\mathcal{N}_{\omega}=\mathcal{O}_{\mathbb{P}^{1}}(n)$. Even if $\mathcal{N}_{\omega}=\mathcal{O}_{\mathbb{P}^{1}}$ for all $\omega \in \mathscr{P}$ of dimension one, $\mathcal{L S}_{X_{0}(B, \mathscr{P}, s)}$ may be non-trivial thanks to non-trivial open gluing data.

In the example given in the introduction, $n=4$ for each edge. Let $\mathcal{X}_{0}$ carry the pull-back of the divisorial log structure $\mathcal{X}_{0} \subseteq \mathcal{X}$. Then the map $\mathcal{X}_{0}^{\dagger} \rightarrow$ Spec $k^{\dagger}$ fails to be log smooth precisely at the $24=4 \times 6$ points where the total space $\mathcal{X}$ is singular, as the log structure is not even fine at these points (Example 3.6). These 24 points are the zeros of a section of the local $T^{1}$ sheaf of $\mathcal{X}_{0}$.

\section{Toric degenerations}

We are now ready to study the fundamental objects of interest in our program. These will be certain degenerations of Calabi-Yau manifolds whose central fibres are of the form $X_{0}(B, \mathscr{P}, s)$. In addition, these fibres come along with natural log structures of the form studied in the previous section.

We gave a definition of toric degeneration in [19], Section 1; the one given here is slightly more general. From now on, we will always work over an algebraically closed field $k$. The algebraically closed hypothesis could be removed from this section at the expense of a bit of additional complexity, but it's vital in the proof of Theorem 5.2.

Definition 4.1. Let $R$ be a discrete valuation ring with residue class field $k$. A toric degeneration of Calabi-Yau varieties over $R$ is a proper 
normal algebraic space $\mathcal{X}$ flat over $\mathcal{S}:=\operatorname{Spec} R$ satisfying the following properties:

1) The generic fibre $\mathcal{X}_{\eta}$ is an irreducible normal variety over $\eta$.

2) If $\nu: \tilde{\mathcal{X}}_{0} \rightarrow \mathcal{X}_{0}$ is the normalization, then $\tilde{\mathcal{X}}_{0}$ is a disjoint union of toric varieties, the conductor scheme $C \subseteq \tilde{\mathcal{X}}_{0}$ is reduced and the map $C \rightarrow \nu(C)$ is unramified and generically two-to-one. The square

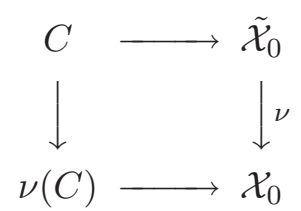

is cartesian and cocartesian.

3) $\mathcal{X}_{0}$ is Gorenstein, and the conductor locus $C$ restricted to each irreducible component of $\tilde{\mathcal{X}}_{0}$ is the union of all toric Weil divisors. (By the discussion before Theorem 2.39, this a substitute for the statement $\nu^{*} \omega_{\mathcal{X}_{0}} \cong \mathcal{O}_{\tilde{\mathcal{X}}_{0}}$.)

4) There exists a closed subset $Z \subseteq \mathcal{X}$ of relative codimension $\geq 2$ such that $Z$ satisfies the following properties: $Z$ does not contain the image under $\nu$ of any toric stratum of $\tilde{\mathcal{X}}_{0}$, and for any geometric point $\bar{x} \rightarrow \mathcal{X} \backslash Z$, there is an étale neighbourhood $U_{\bar{x}} \rightarrow \mathcal{X} \backslash Z$ of $\bar{x}$, an affine toric variety $Y_{\bar{x}}$, a regular function $f_{\bar{x}}$ on $Y_{\bar{x}}$ given by a monomial, a choice of uniformizing parameter of $R$ giving a map $k[\mathbb{N}] \rightarrow R$, and a commutative diagram

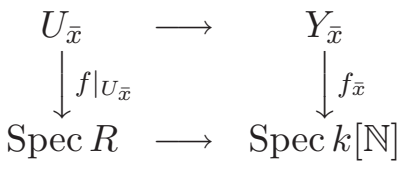

such that the induced map $U_{\bar{x}} \rightarrow \operatorname{Spec} R \times_{\operatorname{Spec} k[\mathbb{N}]} Y_{\bar{x}}$ is smooth. Furthermore, $f_{\bar{x}}$ vanishes on each toric divisor of $Y_{\bar{x}}$.

Geometrically (2) and (3) say that the central fibre is obtained from a disjoint union of toric varieties by identifying pairs of irreducible toric Weil divisors. Glued Weil divisors may lie on the same toric variety, but it is not permitted to glue an irreducible toric Weil divisor to itself (no pinch points). The restriction of $\nu$ to a toric stratum in $\tilde{\mathcal{X}}_{0}$ is finite and generically injective, hence the normalization of its image. We refer to these images in $\mathcal{X}_{0}$ also as toric strata.

\section{Example 4.2.}

1) A straightforward source of toric degenerations are degenerations of hypersurfaces in toric varieties. As in Example 1.18, let $\Xi$ be a reflexive polytope with $0 \in \Xi$ the unique interior point, and let $\Xi^{*} \subseteq$ $N_{\mathbb{R}}$ be the Batyrev dual of $\Xi$ (Example 1.53). Let $\left(\mathbb{P}_{\Xi^{*}}, \mathcal{O}_{\mathbb{P}_{\Xi^{*}}}(1)\right)$ be the projective toric variety with Newton polytope $\Xi^{*}$. Define a family 
$\mathcal{X} \subseteq \mathbb{P}_{\Xi^{*}} \times \mathcal{S}$ with $\mathcal{S}=\operatorname{Spec} k[t]_{(t)}$ by the equation

$$
z^{0}+\sum_{n \in \Xi^{*} \cap N} a_{n} t z^{n}=0
$$

here $z^{n}$ run over a basis of sections of $\mathcal{O}_{\mathbb{P}_{\Xi^{*}}}(1)$. The coefficients $a_{n} \in k$ are chosen to be general. The section $z^{0}$ vanishes precisely once on each toric divisor. It is then easy to check $\mathcal{X} \rightarrow \mathcal{S}$ is a toric degeneration.

We will discuss in Example 4.29 below how exactly this toric degeneration relates to the toric polyhedral decomposition of $\partial \Xi$ introduced in Example 1.23, (2).

2) Here is an example due to Aspinwall and Morrison [4]. Take a family $\mathcal{Y} \subseteq \mathbb{P}^{4} \times \mathcal{S}, \mathcal{S}=\operatorname{Spec} k[t]_{(t)}$, given by the equation

$$
t\left(z_{0}^{5}+\cdots+z_{4}^{5}\right)+z_{0} z_{1} z_{2} z_{3} z_{4}=0 .
$$

We then divide $\mathcal{Y}$ by the group action of $\mathbb{Z}_{5} \times \mathbb{Z}_{5}$, with generators acting by $\left(z_{0}, \ldots, z_{4}\right) \mapsto\left(z_{0}, \xi z_{1}, \ldots, \xi^{4} z_{4}\right)$ and $\left(z_{0}, \ldots, z_{4}\right) \mapsto\left(z_{1}, \ldots, z_{4}, z_{0}\right)$. Here $\xi$ is a primitive fifth root of unity. Set $\mathcal{X}=\mathcal{Y} / \mathbb{Z}_{5} \times \mathbb{Z}_{5}$; then $\mathcal{X} \rightarrow \mathcal{S}$ is a toric degeneration.

This example will turn out to be related to the affine manifold from Example 1.19.

For us, the key structure of a toric degeneration is the central fibre, $\mathcal{X}_{0}$, along with the $\log$ structure on $\mathcal{X}_{0}$ obtained by pulling back the log structure on $\mathcal{X}$ induced by the inclusion $\mathcal{X}_{0} \subseteq \mathcal{X}$ as in Example 3.2. Thus we can define the logarithmic analogue of a Calabi-Yau variety as follows.

Definition 4.3. A toric log Calabi-Yau space is a proper reduced logarithmic space $X^{\dagger}$ along with a morphism of log spaces $X^{\dagger} \rightarrow$ Spec $k^{\dagger}$ to the standard log point with the following properties:

1) If $\nu: \tilde{X} \rightarrow X$ is the normalization, then $\tilde{X}$ is a disjoint union of toric varieties, the conductor scheme $C \subseteq \tilde{X}$ is reduced and the map $C \rightarrow \nu(C)$ is generically two-to-one and unramified. The square formed by $C, \nu(C), \tilde{X}$ and $X$ is cartesian and cocartesian.

2) $X$ is Gorenstein, and the conductor locus $C$ restricted to each irreducible component of $\tilde{X}$ is the union of all toric Weil divisors. (As before, this a substitute for the statement $\nu^{*} \omega_{X} \cong \mathcal{O}_{\tilde{X}}$.)

3) There exists a closed subset $Z \subseteq \operatorname{Sing}(X)$ of relative codimension $\geq 2$ and not containing any toric stratum (called the log-singular set), such that $X^{\dagger} \backslash Z$ is a fine $\log$ scheme and $X^{\dagger} \backslash Z \rightarrow$ Spec $k^{\dagger}$ is $\log$ smooth. Furthermore $\overline{\mathcal{M}}_{X \backslash Z}$ is a sheaf of toric monoids, and if $\bar{\rho} \in \Gamma\left(X, \overline{\mathcal{M}}_{X}\right)$ is the image of $1 \in \mathbb{N}$ under the structure map of $X^{\dagger} \rightarrow$ Spec $k^{\dagger}$, then the function $z^{\bar{\rho}}$ vanishes precisely once along each toric Weil divisor of Spec $k\left[\overline{\mathcal{M}}_{X, \bar{x}}\right]$ for every geometric point $\bar{x} \rightarrow X \backslash Z$. 
We say $X_{1}^{\dagger} \rightarrow$ Spec $k^{\dagger}$ and $X_{2}^{\dagger} \rightarrow$ Spec $k^{\dagger}$ are isomorphic toric log Calabi-Yau spaces if there is an isomorphism $\varphi: X_{1} \rightarrow X_{2}$ of algebraic spaces over $k$ lifting to an isomorphism of log spaces $X_{1}^{\dagger} \backslash Z \rightarrow X_{2}^{\dagger} \backslash \varphi(Z)$ over Spec $k^{\dagger}$, for some log-singular set $Z$ for $X_{1}^{\dagger}$.

Remark 4.4. The spaces $X_{0}(B, \mathscr{P}, s)$ look like underlying spaces of a toric log Calabi-Yau space. The main difficulty is to place a log structure on these spaces. This will be done in $\S 5.1$ under the additional hypothesis of $(B, \mathscr{P})$ being simple.

Remark 4.5. We never make use of the $\log$ structure over $Z$, and in fact all relevant information is contained completely in the complement of any $Z$ with the stated properties. This is due to the fact that there is a canonical extension of the $\log$ structure on $X \backslash Z$ to $X$ that retrieves the original $\log$ structure at all $\log$-smooth points: Let $j: X \backslash Z \rightarrow X$ be the inclusion. Since $X$ is Gorenstein and $Z$ is of codimension $\geq 2, j_{*} \mathcal{O}_{X \backslash Z}=$ $\mathcal{O}_{X}$. Thus the $\log$ structure on $X \backslash Z$ extends uniquely to a log structure on $X$ with monoid sheaf $j_{*} \mathcal{M}_{X \backslash Z}$. If $Z$ does not contain any toric stratum the canonical map $\mathcal{M}_{X} \rightarrow j_{*} \mathcal{M}_{X \backslash Z}$ induces an isomorphism of ghost sheaves wherever $\mathcal{M}_{X}$ is fine, hence an isomorphism of log structures.

A canonical, minimal choice for $Z$ is the closed subspace where $(X$, $\left.j_{*} \mathcal{M}_{X \backslash Z}\right) \rightarrow$ Spec $k^{\dagger}$ is not a log smooth morphism of fine log spaces.

Proposition 4.6. If $\mathcal{X} \rightarrow \operatorname{Spec} R$ is a toric degeneration, let $\mathcal{X}^{\dagger}$ and Spec $R^{\dagger}$ be the log structures induced by the inclusions $X=\mathcal{X}_{0} \subseteq \mathcal{X}$ and Spec $k \subseteq \operatorname{Spec} R$ as in Example 3.2. Let $X^{\dagger}$ be the induced log structure on $X$. Then the induced morphism $X^{\dagger} \rightarrow$ Spec $k^{\dagger}$ gives $X^{\dagger}$ the structure of a toric log Calabi-Yau space.

Proof. Clearly $X$ satisfies conditions (1) and (2) of Definition 4.3, as these are (2) and (3) of Definition 4.1. Note that we obtain a morphism of $\log$ spaces $\mathcal{X}^{\dagger} \rightarrow$ Spec $R^{\dagger}$ induced by pull-back of functions, as $\mathcal{M}_{\mathcal{X}}$ and $\mathcal{M}_{\text {Spec } R}$ are contained in the structure sheaves of $\mathcal{X}$ and $\operatorname{Spec} R$ respectively. This morphism then restricts to a morphism of log spaces $X^{\dagger} \rightarrow \operatorname{Spec} k^{\dagger}$.

Now if $P$ is a toric monoid, the log structure on Spec $k[P]$ induced by the chart $P \rightarrow k[P]$ coincides with the log structure induced by the inclusion $\partial \operatorname{Spec} k[P] \hookrightarrow \operatorname{Spec} k[P]$, where by $\partial \operatorname{Spec} k[P]$ we mean the union of all toric divisors of Spec $k[P]$. On the other hand, in (4) of Definition 4.1 , because $f_{\bar{x}}$ vanishes to order 1 on each toric divisor of $Y_{\bar{x}}, f_{\bar{x}}^{-1}(0)=\partial Y_{\bar{x}}$. Thus if $Y_{\bar{x}}=\operatorname{Spec} k\left[P_{\bar{x}}\right]$, the induced map $P_{\bar{x}} \rightarrow \mathcal{O}_{U_{\bar{x}}}$ gives a chart for the $\log$ structure on $U_{\bar{x}}$ induced from $\mathcal{X}^{\dagger}$. Thus we see that condition (4) of Definition 4.1 implies $\mathcal{X}^{\dagger} \backslash Z \rightarrow$ Spec $R^{\dagger}$ is log smooth, and hence by restriction $X^{\dagger} \backslash Z$ is also log smooth. q.e.d. 
Remark 4.7. In the quartic example of the introduction, the minimal singular set $Z$ coincides with the singular locus of the total space of $\mathcal{X}$, but this is not true more generally, as $\mathcal{X}$ may have toric singularities. However, the singular set $Z$ is the set of points where these singularities are somehow "worse."

We will never be interested in the $\log$ structure at the points of $Z$ itself, hence the definition of isomorphism of log Calabi-Yau spaces is independent of the log structure along $Z$.

Toric strata also have a characterization in terms of the ghost sheaf.

Lemma 4.8. Let $X$ be a toric log Calabi-Yau space. A geometric point $\bar{x} \rightarrow X \backslash Z$ is the geometric generic point of a toric stratum of $X$ if and only if $\operatorname{rank}\left(\overline{\mathcal{M}}_{X, \bar{x}}^{\mathrm{gp}}\right)=\operatorname{dim} \mathcal{O}_{X, \bar{x}}+1$.

Proof. Log smoothness gives a smooth map

$$
\pi: U \longrightarrow V:=\operatorname{Spec} k\left[\overline{\mathcal{M}}_{X, \bar{x}}\right] /\left(z^{\bar{\rho}_{\bar{x}}}\right)
$$

from an étale neighbourhood $U$ of $\bar{x}$. Since $V$ is a toric subscheme inside a toric variety it also comes with a notion of toric strata, which are the intersections of the irreducible components of $V$. Define toric strata of $U$ as the components of preimages of toric strata under $U \rightarrow V$. Now by condition (2) of Definition 4.3, étale locally toric strata of $X$ are also precisely the intersections of irreducible components of $X$. Therefore, toric strata in $U$ map to toric strata in $X$ and it suffices to prove the statement for $U$ instead of $X$.

Since $\overline{\mathcal{M}}_{X, \bar{x}}$ has no non-trivial invertibles there is a distinguished closed point $0 \in V$, the zero-dimensional toric stratum. Let $\eta \in \pi^{-1}(0)$ be the generic point of the connected component of $\pi^{-1}(0)$ containing $\bar{x}$. Then $\overline{\mathcal{M}}_{X, \bar{x}}=\overline{\mathcal{M}}_{X, \bar{\eta}}$, and the connected component of $\pi^{-1}(0)$ containing $\bar{x}$ is the minimal toric stratum of $U$ containing $\bar{x}$. Thus $\bar{x}$ is the generic point of a toric stratum if and only if $\bar{x}=\bar{\eta}$. By the dimension formula for smooth morphisms this is the case if and only if $\operatorname{dim} \mathcal{O}_{X, \bar{x}}=$ $\operatorname{dim}(V)$. The proof is finished by noting $\operatorname{dim}(V)=\operatorname{rank}\left(\overline{\mathcal{M}}_{X, \bar{x}}^{\mathrm{gp}}\right)-1$. q.e.d.

4.1. The dual intersection complex. Given a toric log Calabi-Yau space $X^{\dagger}$, we now reverse the gluing procedures described earlier in this paper in order to construct an affine manifold with singularities. We begin by defining a category analogous to $\underline{\mathrm{Cat}}(\mathscr{P})$, which we call $\underline{\operatorname{Cat}}(X)$. Write $\tilde{X}=\coprod X_{i}$, with $\nu: \tilde{X} \rightarrow X$ the normalization. The objects of $\underline{\operatorname{Cat}}(X)$ are the strata of $X$, i.e., the set

$$
\operatorname{Strata}(X):=\left\{\nu(S) \mid S \text { is a toric stratum of } X_{i} \text { for some } i\right\} \text {. }
$$

If $S_{1}, S_{2}$ are two strata of $X$, we put $\operatorname{Hom}\left(S_{1}, S_{2}\right)=\emptyset$ if $S_{1} \nsupseteq S_{2}$, $\operatorname{Hom}\left(S_{1}, S_{2}\right)=\{i d\}$ if $S_{1}=S_{2}$. If $S_{1} \supset S_{2}$, let $\nu_{1}: \tilde{S}_{1} \rightarrow S_{1}$ be the 
normalization of $S_{1}$, so $\tilde{S}_{1}$ is a toric variety. Then

$\operatorname{Hom}\left(S_{1}, S_{2}\right)=\left\{\tilde{S}_{2} \mid \tilde{S}_{2}\right.$ is a toric stratum of $\tilde{S}_{1}$ with $\left.\nu_{1}\left(\tilde{S}_{2}\right)=S_{2}\right\}$.

If we have a chain $S_{1} \supset S_{2} \supset S_{3}$, an element of $\operatorname{Hom}\left(S_{1}, S_{2}\right)$ is a stratum $\tilde{S}_{2}$ of $\tilde{S}_{1}$ mapping to $S_{2}$; identifying $\tilde{S}_{2}$ with the normalization of $S_{2}$, an element of $\operatorname{Hom}\left(S_{2}, S_{3}\right)$ can then be identified also as a substratum of $\tilde{S}_{1}$, and this defines composition of morphisms.

Next let LPoly be the category of lattice polytopes, a subcategory of the category of topological spaces, with the objects being polytopes with integral vertices and morphisms $\operatorname{Hom}\left(\sigma_{1}, \sigma_{2}\right)$ being integral affine identifications of $\sigma_{1}$ as a face of $\sigma_{2}$.

We can then define a functor

$$
L P: \underline{\operatorname{Cat}}(X) \longrightarrow \underline{\text { LPoly }}
$$

as follows. If $S \in \operatorname{Strata}(X)$, with generic point $\eta$, then the stalk $\overline{\mathcal{M}}_{X, \bar{\eta}}$ is a monoid with a distinguished element $\bar{\rho}$ specified by the morphism $X^{\dagger} \rightarrow$ Spec $k^{\dagger}: \bar{\rho}$ is the germ of the image of $1 \in \mathbb{N}$ in $\overline{\mathcal{M}}_{X, \bar{\eta}}$. We define $L P(S)$ to be the convex hull of

$$
\left\{\varphi \in \operatorname{Hom}\left(\overline{\mathcal{M}}_{X, \bar{\eta}}, \mathbb{N}\right) \mid \varphi(\bar{\rho})=1\right\}
$$

inside the affine space

$$
\left\{\varphi \in \operatorname{Hom}\left(\overline{\mathcal{M}}_{X, \bar{\eta}}, \mathbb{R}\right) \mid \varphi(\bar{\rho})=1\right\} .
$$

Lemma 4.9. If $S \in \operatorname{Strata}(X)$, then $L P(S)$ is a non-empty lattice polytope.

Proof. Let $P=\overline{\mathcal{M}}_{X, \bar{\eta}}$ for $\eta$ the generic point of $S$, with $\bar{\rho} \in P$ given by the morphism $X^{\dagger} \rightarrow$ Spec $k^{\dagger}$. Then as $P$ is a toric monoid, the toric variety $\operatorname{Spec} k[P]$ is given by a cone $K \subset \operatorname{Hom}(P, \mathbb{R})$, the convex hull of $\operatorname{Hom}(P, \mathbb{N})$. The condition that $\bar{\rho}$ should be 1 on each irreducible component of $X$ implies that $z^{\bar{\rho}} \in k[P]$ vanishes to order 1 on each toric divisor of Spec $k[P]$. But this is saying $\bar{\rho}$ evaluates to 1 on the primitive integral generators of the extremal rays of $K$. Thus $L P(S)$ is just the convex hull of these integral generators, and hence is a (non-empty) lattice polytope.

q.e.d.

Now suppose we are given strata $S_{1} \supset S_{2}$, and an element of $\operatorname{Hom}\left(S_{1}\right.$, $S_{2}$ ), i.e., strata $\tilde{S}_{1} \supset \tilde{S}_{2}$, where $\nu_{1}: \tilde{S}_{1} \rightarrow S_{1}$ is the normalization. We need to define a morphism $L P\left(S_{1}\right) \rightarrow L P\left(S_{2}\right)$. This is done as follows. If $\eta_{1}, \eta_{2}$ are the generic points of $\tilde{S}_{1}, \tilde{S}_{2}$ respectively, there is a welldefined cospecialization map

$$
\overline{\mathcal{M}}_{X, \bar{\eta}_{2}}=\left(\nu_{1}^{*} \overline{\mathcal{M}}_{X}\right)_{\bar{\eta}_{2}} \longrightarrow\left(\nu_{1}^{*} \overline{\mathcal{M}}_{X}\right)_{\bar{\eta}_{1}}=\overline{\mathcal{M}}_{X, \bar{\eta}_{1}} .
$$

Note the identifications on the left and right are canonical, but the map depends on which stratum $\tilde{S}_{2} \subset \tilde{S}_{1}$ dominating $S_{2}$ we have chosen. It is 
easy to see that such a map is a surjection and dually we obtain a map

$$
\operatorname{Hom}\left(\overline{\mathcal{M}}_{X, \bar{\eta}_{1}}, \mathbb{N}\right) \longrightarrow \operatorname{Hom}\left(\overline{\mathcal{M}}_{X, \bar{\eta}_{2}}, \mathbb{N}\right)
$$

which identifies the first monoid as a face of the second. In particular, we then obtain a map $L P\left(S_{1}\right) \rightarrow L P\left(S_{2}\right)$ identifying the first polytope as a face of the second. It is clear this construction is compatible with composition of morphisms in $\operatorname{Strata}(X)$.

We can now construct the dual intersection complex $B$ of $X$ as a union of polyhedra, explicitly $\lim L P$ in the category of topological spaces.

Proposition 4.10. If $\operatorname{dim} X=n$, then $B$ is an $n$-dimensional manifold.

We will first need the following Lemma.

Lemma 4.11. Let $X_{1}$ and $X_{2}$ be two affine toric varieties over $k$, defined by strictly convex cones $\sigma_{1}, \sigma_{2} \subseteq M_{\mathbb{R}}$, with $\operatorname{dim} \sigma_{1}=\operatorname{dim} \sigma_{2}=$ $\operatorname{dim} M_{\mathbb{R}}$. Let $x_{i} \in X_{i}$ be the zero-dimensional torus orbit. Suppose $X_{1}$ and $X_{2}$ are étale locally isomorphic at $x_{i}$, i.e., there is an isomorphism of strictly Henselian local $k$-algebras $\psi: \mathcal{O}_{X_{1}, \bar{x}_{1}} \rightarrow \mathcal{O}_{X_{2}, \bar{x}_{2}}$. Suppose further that this isomorphism preserves toric strata, i.e., it takes the ideal of a toric stratum in $\mathcal{O}_{X_{1}, \bar{x}_{1}}$ to the ideal of a toric stratum in $\mathcal{O}_{X_{2}, \bar{x}_{2}}$. Then there exists an element $\varphi: M \rightarrow M$ of $G L_{n}(\mathbb{Z})$ such that $\varphi\left(\sigma_{1}\right)=\sigma_{2}$, and which is combinatorially compatible with $\psi$, i.e., if $\psi$ identifies the ideals of two toric strata, then $\varphi$ identifies the corresponding cones.

Proof. Let $P_{i}=\sigma_{i}^{\vee} \cap N$ be the monoid defining $X_{i}$. Then if $X_{i}^{\dagger}$ is the log structure induced by the inclusion $\partial X_{i} \subseteq X_{i}$, then $\overline{\mathcal{M}}_{X_{i}, \bar{x}_{i}} \cong$ $P_{i}$. It is then clear that the isomorphism $\psi$ induces an isomorphism $\overline{\mathcal{M}}_{X_{2}, \bar{x}_{2}} \stackrel{\cong}{\longrightarrow} \overline{\mathcal{M}}_{X_{1}, \bar{x}_{1}}$, and then an isomorphism $M \cong \operatorname{Hom}\left(P_{1}, \mathbb{Z}\right) \rightarrow M \cong$ $\operatorname{Hom}\left(P_{2}, \mathbb{Z}\right)$ taking $\sigma_{1} \subseteq M_{\mathbb{R}}$ to $\sigma_{2} \subseteq M_{\mathbb{R}}$ as desired. q.e.d.

Proof of Proposition 4.10. The vertices of $B$ correspond to irreducible components of $X$. It is clearly enough to show $B$ is an $n$-dimensional manifold in a neighbourhood of each vertex, since $B$ behaves uniformly along each face.

Focusing on one vertex $v$ of $B$, there is a corresponding toric variety $X_{v}$. Now for any zero-dimensional stratum $x \in X_{v}$, there is a monoid $\overline{\mathcal{M}}_{X, \nu(\bar{x})}=: P_{\bar{x}}$. Lemma 4.8 shows $\operatorname{dim} k\left[P_{\bar{x}}\right] /\left(z^{\bar{\rho}_{\bar{x}}}\right)=n$. Let $K_{\bar{x}}$ be the convex hull of $\operatorname{Hom}\left(P_{\bar{x}}, \mathbb{N}\right)$ in $\operatorname{Hom}\left(P_{\bar{x}}, \mathbb{R}\right)$; this is the cone defining the toric variety $\operatorname{Spec} k\left[P_{\bar{x}}\right]$. It is then clear that $\operatorname{Hom}\left(P_{\bar{x}}, \mathbb{R}\right) \cong \mathbb{R}^{n+1}$ and $K_{\bar{x}}$ is an $n+1$-dimensional cone, by the previous observations. Thus $L P(\nu(\bar{x}))$ is in particular a $n$-dimensional lattice polytope.

If $\eta$ is the generic point of $\nu\left(X_{v}\right)$, then the cospecialization map induced by $\bar{x} \rightarrow X_{v}, \overline{\mathcal{M}}_{X, \nu(\bar{x})} \rightarrow \overline{\mathcal{M}}_{X, \bar{\eta}}$, is dual to the inclusion of some 
extremal ray $R_{\bar{x}} \subseteq K_{\bar{x}}$. The tangent wedge of $L P(\nu(\bar{x}))$ at the vertex $p$ is just the cone $K_{\bar{x}}\left(R_{\bar{x}}\right)=\left(K_{\bar{x}}+\mathbb{R} R_{\bar{x}}\right) / \mathbb{R} R_{\bar{x}}$, and by Lemma 4.11, this cone is the same as the cone corresponding to the stratum $x \in X_{v}$ in the fan $\Sigma_{X_{v}}$ defining $X_{v}$, up to integral linear transformations. It is then easy to see that all the cones $K_{\bar{x}}\left(R_{\bar{x}}\right)$ fit together to give, at least topologically, the fan $\Sigma_{X_{v}}$, as $\{x\}$ runs over all zero-dimensional strata of $X_{v}$. But this is the local picture of $B$ at $v$, so $B$ is a manifold in a neighbourhood of $v$.

q.e.d.

We can now describe an integral affine structure with singularities on $B$ with a polyhedral decomposition $\mathscr{P}$. Here

$$
\mathscr{P}=\{\text { image of } L P(S) \text { in } B=\underset{\lim }{\longrightarrow} L P \mid S \in \operatorname{Strata}(X)\} .
$$

By Construction 1.26, it is enough to give a fan structure at each vertex of $\mathscr{P}$ compatible with the polyhedral decomposition. But we have already done this in the proof of Proposition 4.10: There is a combinatorial identification of $\mathscr{P}$ in a neighbourhood of a vertex $v$ with the fan $\Sigma_{X_{v}}$. This gives the fan structure.

Thus we have obtained an integral affine manifold $B$ with singularities along the discriminant locus $\Delta^{\prime}$ given in Construction 1.26. We now observe:

Proposition 4.12. $\mathscr{P}$ is a toric polyhedral decomposition of $B$.

Proof. The only thing to check is that $\mathscr{P}$ is toric, since it is a polyhedral decomposition by construction. We will show this by applying Proposition 1.32.

To do this, let $\tau \in \mathscr{P}$ correspond to some stratum $S$, which is the image of toric strata $S_{1} \subseteq X_{v_{1}}, \ldots, S_{m} \subseteq X_{v_{m}}$, where $v_{1}, \ldots, v_{m}$ are the (possibly non-distinct) vertices of $\tau$. Then $\nu$ yields canonical isomorphisms between the $S_{i}$ which preserve the toric strata of the $S_{i}$; thus these isomorphisms are equivariant under the torus action on $\amalg X_{v_{i}}$. So the fans determining $S_{1}, \ldots, S_{m}$ can be identified. Specifically, if $\Sigma_{v_{i}}$ defines $X_{v_{i}}$, living naturally in $\Lambda_{\mathbb{R}, v_{i}}$, and $\tau_{v_{i}} \in \Sigma_{v_{i}}$ is the cone corresponding to $S_{i}$, then the fan for $S_{i}$ is $\Sigma_{v_{i}}\left(\tau_{v_{i}}\right)$, living naturally in $\Lambda_{\mathbb{R}, v_{i}} / \Lambda_{\tau, \mathbb{R}}$

Then parallel translation from $v_{i}$ to $v_{j}$ along some path identifies

$$
\Lambda_{\mathbb{R}, v_{i}} / \Lambda_{\tau, \mathbb{R}} \text { and } \Lambda_{\mathbb{R}, v_{j}} / \Lambda_{\tau, \mathbb{R}}
$$

in such a way that the fans $\Sigma_{v_{i}}\left(\tau_{v_{i}}\right)$ and $\Sigma_{v_{j}}\left(\tau_{v_{j}}\right)$ are identified. There is a unique such identification, and hence this is independent of the path chosen. Thus it follows, for any loop $\gamma$ based at $y \in \operatorname{Int}(\tau) \backslash \Delta^{\prime}$ in a sufficiently small neighbourhood $U_{\tau}$ of $\operatorname{Int}(\tau)$, that $\tilde{\rho}(\gamma)$ acts trivially on $\Lambda_{\mathbb{R}, y} / \Lambda_{\tau, \mathbb{R}}$, i.e.,

$$
(\tilde{\rho}(\gamma)-I)\left(\Lambda_{\mathbb{R}, y}\right) \subseteq \Lambda_{\tau, \mathbb{R}}
$$

as desired. 
Definition 4.13. If $X^{\dagger}$ is a toric log Calabi-Yau space, then the integral affine manifold with singularities $B$ along with the polyhedral decomposition $\mathscr{P}$ constructed above is the dual intersection complex of $X^{\dagger}$.

Theorem 4.14. Let $X^{\dagger}$ be a toric log Calabi-Yau space, and let $(B, \mathscr{P})$ be the dual intersection complex. Then there exist open gluing data $s$ for $\mathscr{P}$ over $k$ such that

$$
X \cong X_{0}(B, \mathscr{P}, s) .
$$

Proof. Let $X_{\tau}$ be as usual for $\tau \in \mathscr{P}$. Then by construction, there is an isomorphism $\psi_{\tau}: X_{\tau} \rightarrow S_{\tau}$, where $S_{\tau}$ is the normalization of the stratum of $X$ corresponding to $\tau$. Furthermore, this isomorphism can be chosen to identify the toric strata of $X_{\tau}$ with the strata of $S_{\tau}$. Then $\psi_{\tau}$ is well-defined up to the action of $\mathcal{Q}_{\tau} \otimes \mathbb{G}_{m}(k)$ on $X_{\tau}$. Fix such choices. In particular, for every $e: \tau \rightarrow \sigma$, we then obtain $\bar{s}_{e} \in \mathcal{Q}_{\sigma} \otimes \mathbb{G}_{m}(k)$ such that the diagram

$$
\begin{array}{ccc}
X_{\sigma} \stackrel{\psi_{\sigma}}{\longrightarrow} & S_{\sigma} \\
\downarrow & & \\
\downarrow & & \\
X_{\tau}(e) \circ \bar{s}_{e} & \downarrow & \downarrow \\
\psi_{\tau} & \stackrel{\psi_{\tau}}{\longrightarrow} & S_{\tau}
\end{array}
$$

commutes. Then $\bar{s}=\left(\bar{s}_{e}\right)$ forms closed gluing data, and $\lim F_{\operatorname{Spec} k, \bar{s}}=$ $X$. Indeed, there is a canonical map $\lim F_{\text {Spec } k, \bar{s}} \rightarrow X$ induced by the system of composed morphisms $X_{\tau} \rightarrow S_{\tau} \rightarrow X$. Since $\lim F_{\text {Spec } k, \bar{s}}$ glues together the components $X_{v}$ for $v$ a vertex in $\mathscr{P}$ in a normal crossings way in codimension one, we must have $\lim F_{\text {Spec } k, \bar{s}} \rightarrow X$ being an isomorphism in codimension one. On the other hand, as $X$ is assumed to be Gorenstein, and in particular $S_{2}$, in fact this is an isomorphism everywhere by [41], Proposition 2.2. We then have to show this gluing arises via open gluing data.

First, we show $X$ has an étale open cover by sets of the form $V(\sigma)$ for $\sigma \in \mathscr{P}_{\max }$. Fix $\sigma \in \mathscr{P}_{\max }$, and let $\bar{x} \rightarrow X$ be the corresponding zero-dimensional stratum. Then we have a chart

$$
P_{\sigma}:=\overline{\mathcal{M}}_{X, \bar{x}} \longrightarrow \mathcal{O}_{X, \bar{x}}
$$

giving the log structure at $x$. Now for each $e \in \coprod_{\substack{\tau \in \mathscr{P} \\ \tau \neq \sigma}} \operatorname{Hom}(\tau, \sigma)$, we have an affine open set $V_{e}$ of $X_{\tau}$ corresponding to the cone $K_{\tau}$ in $\Sigma_{\tau}$, and $V(\sigma)=\lim V_{e}$ with respect to the canonical directed system $F_{\sigma}(f)$ : $V_{e_{2}} \rightarrow V_{e_{1}}$ over all commutative diagrams

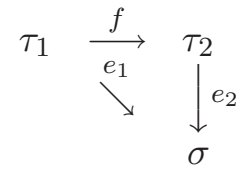


On the other hand, let $V^{\prime}(\sigma)$ be the limit with respect to the system

$$
F_{\sigma}(\bar{s})(f): V_{e_{2}} \longrightarrow V_{e_{1}}
$$

given by $F_{\sigma}(\bar{s})(f)=F_{\sigma}(f) \circ \bar{s}_{f}$. Using the universal property of colimits it is straightforward to show that the canonical map $V^{\prime}(\sigma) \rightarrow X$ is étale. It thus remains to produce an isomorphism of $V(\sigma)$ and $V^{\prime}(\sigma)$.

To do so, note that $V_{e} \cong \operatorname{Spec} k\left[P_{e}\right]$, where $P_{e}$ is the face of $P_{\sigma}$ corresponding to $e$. Thus by composing the chart $P_{\sigma} \rightarrow \mathcal{O}_{X, \bar{x}}$ with the map $\mathcal{O}_{X, \bar{x}} \rightarrow \mathcal{O}_{V_{e}, \bar{x}}$ induced by the composition $V_{e} \rightarrow V^{\prime}(\sigma) \rightarrow X$, we obtain a map of monoids $\phi_{e}: P_{e} \rightarrow \mathcal{O}_{V_{e}, \bar{x}}$ of the form $\phi_{e}(p)=h_{p} z^{p}$ for $h_{p} \in \mathcal{O}_{V_{e}, \bar{x}}^{\times}$; in particular, $p \mapsto h_{p}$ is a map of monoids $P_{e} \rightarrow \mathcal{O}_{V_{e}, \bar{x}}^{\times}$. Composing this map with the residue map

$$
\mathcal{O}_{V_{e}, \bar{x}}^{\times} \longrightarrow \mathcal{O}_{V_{e}, \bar{x}}^{\times} /\left(1+\mathbf{m}_{\bar{x}}\right) \cong k^{\times},
$$

we get maps

$$
\bar{\phi}_{e}: P_{e} \longrightarrow k\left[P_{e}\right]
$$

given by

$$
p \longmapsto\left(h_{p} \bmod 1+\mathbf{m}_{\bar{x}}\right) \cdot z^{p} .
$$

This is still a map of monoids; what we are doing is removing the "nonconstant" part of the log structure. Furthermore, given $f$ as in (4.1), we necessarily have a commutative diagram

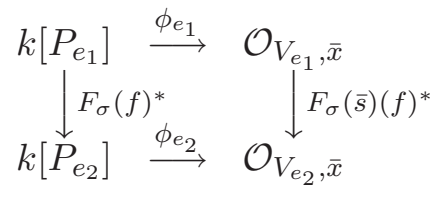

hence a commutative diagram

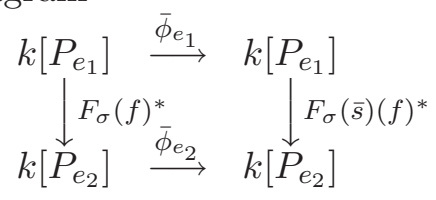

and hence an isomorphism of directed systems inducing an isomorphism $\phi: V^{\prime}(\sigma) \rightarrow V(\sigma)$, as desired.

Now let $U=\coprod_{\sigma \in \mathscr{P} \max } V(\sigma)$. Then we obtain an étale open cover $\psi: U \rightarrow X$ which is the composition of $\phi^{-1}$ and the natural map $V^{\prime}(\sigma) \rightarrow X$ on each $V(\sigma)$. We have an étale equivalence relation $\mathfrak{R}=$ $U \times{ }_{X} U \subseteq U \times U$.

To obtain the open gluing data from this, choose for each $\omega \in \mathscr{P}$ a morphism $e_{\omega}: \omega \rightarrow \sigma$, for some $\sigma \in \mathscr{P}_{\max }$. Set $s_{e_{\omega}}=1$. If $e: \omega \rightarrow \sigma^{\prime}$ is any other morphism with $\sigma^{\prime} \in \mathscr{P}_{\max }$, we obtain canonically $V(\omega) \subseteq$ $V(\sigma)$ and $V(\omega) \subseteq V\left(\sigma^{\prime}\right)$, with a canonical identification given by $\Phi_{e_{\omega} e}$ as in Section 2. Then $\mathfrak{R} \cap\left(\left(V(\omega) \subseteq V\left(\sigma^{\prime}\right)\right) \times(V(\omega) \subseteq V(\sigma)) \subseteq V\left(\sigma^{\prime}\right) \times\right.$ $V(\sigma)$ can be viewed as the graph of a morphism, and by restricting to irreducible components it is easy to see this morphism is necessarily of 
the form $\Phi_{e_{\omega} e} \circ s_{e}$ for some $s_{e} \in P \breve{M}(\omega)$. This defines $s_{e}$ whenever $e$ is a morphism to a maximal facet. Then given a diagram (4.1), we define

$$
s_{f}=\left.s_{e_{2}}^{-1}\right|_{\tau_{1}} \cdot s_{e_{1}} \text {. }
$$

We need to check this is well-defined. Given

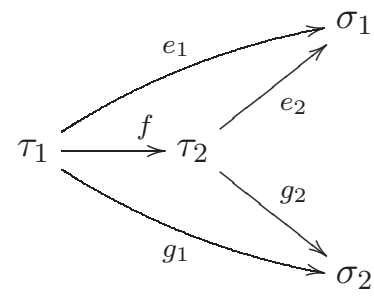

it follows by transitivity of $\Re$ that

$$
\mathfrak{R} \cap\left(\left(V\left(\tau_{1}\right) \subseteq V\left(\sigma_{1}\right)\right) \times\left(V\left(\tau_{1}\right) \subseteq V\left(\sigma_{2}\right)\right)\right)
$$

is the graph of $s_{g_{1}}^{-1} \circ \Phi_{g_{1} e_{1}} \circ s_{e_{1}}$ while

$$
\mathfrak{R} \cap\left(\left(V\left(\tau_{2}\right) \subseteq V\left(\sigma_{1}\right)\right) \times\left(V\left(\tau_{2}\right) \subseteq V\left(\sigma_{2}\right)\right)\right)
$$

is the graph of $s_{g_{2}}^{-1} \circ \Phi_{g_{2} e_{2}} \circ s_{e_{2}}$. These graphs agree on $\left(V\left(\tau_{1}\right) \subseteq V\left(\sigma_{1}\right)\right) \times$ $\left(V\left(\tau_{1}\right) \subseteq V\left(\sigma_{2}\right)\right)$, and hence

$$
\left.\left(s_{e_{2}}^{-1} s_{g_{2}}\right)\right|_{\tau_{1}}=s_{e_{1}}^{-1} s_{g_{1}},
$$

proving well-definedness. Then $\left(s_{e}\right)$ forms open gluing data, and by construction it is clear that $X \cong X_{0}(B, \mathscr{P}, s)$.

q.e.d.

Corollary 4.15. Let $X^{\dagger}$ be a toric log Calabi-Yau space with dual intersection complex $(B, \mathscr{P}), X=X_{0}(B, \mathscr{P}, s)$ for some open gluing data $s$, and let $Z \subseteq X$ be the singular set. If $X_{0}(B, \mathscr{P}, s)^{g}$ denotes the ghost structure on $X_{0}(B, \mathscr{P}, s)$ of Example 3.17, then $X^{\dagger}$ is of ghost type $X_{0}(B, \mathscr{P}, s)^{g}$ on $X \backslash Z$, and thus the log smooth structure on $X \backslash Z$ is induced by a section $f \in \Gamma\left(X \backslash Z, \mathcal{L S}_{X_{0}(B, \mathscr{P}, s)}\right)$. Conversely, given an integral affine manifold $B$ with toric polyhedral decomposition $\mathscr{P}, s$ open gluing data, and if $Z \subseteq X_{0}(B, \mathscr{P}, s)$ is a closed subset of codimension $\geq 2$ not containing any toric stratum, then any section $f \in \Gamma\left(X_{0}(B, \mathscr{P}, s) \backslash Z, \mathcal{L S}_{X_{0}(B, \mathscr{P}, s)}\right)$ induces a toric log CalabiYau space structure on $X_{0}(B, \mathscr{P}, s)$.

Proof. By log smoothness the type of log structure of $X^{\dagger}$ along a toric stratum minus lower-dimensional strata is constant and may hence be read off at the generic point of the stratum. Thus the statement follows from the construction of the dual intersection complex, Example 3.17, and Proposition 3.20. We note for the second part that $f \in \Gamma\left(X_{0}(B, \mathscr{P}, s) \backslash Z, \mathcal{L S}_{X_{0}(B, \mathscr{P}, s)}\right)$ defines a log smooth structure on $X_{0}(B, \mathscr{P}, s) \backslash Z$, which is all we need by Remark 4.5.

q.e.d. 
4.2. Polarized log Calabi-Yau spaces and the intersection complex. Let $X^{\dagger}$ be a toric log Calabi-Yau space with a line bundle $\mathcal{L}$. Then the dual intersection complex $(B, \mathscr{P})$ carries a multi-valued piecewise linear function constructed as follows. For every $\sigma \in \mathscr{P}$, we have the map $q_{\sigma}: X_{\sigma} \rightarrow X$. Pulling back $\mathcal{L}$ to $X_{\sigma}$ gives a line bundle on the toric variety $X_{\sigma}$, and hence an integral piecewise linear function on the fan $\Sigma_{\sigma}$, well-defined up to linear functions. Recall that if $m_{1}, \ldots, m_{p}$ are primitive integral generators of the rays of $\Sigma_{\sigma}$, with corresponding toric divisors $D_{1}, \ldots, D_{p}$, then the toric divisor $\sum a_{i} D_{i}$ corresponds to the piecewise linear function $\varphi_{\sigma}$ on $\Sigma_{\sigma}$ with $\varphi_{\sigma}\left(m_{i}\right)=a_{i}$. By pulling this function back to $U_{\sigma} \subseteq B$ via $S_{\sigma}$ of Definition 1.22 , (5), one obtains piecewise linear functions on the open covering $\left\{U_{\sigma} \mid \sigma \in \mathscr{P}\right\}$ of $B$. It is easy to see these functions differ only by linear functions on intersections, and thus we obtain a multi-valued piecewise linear function $\varphi_{\mathcal{L}}$ with integral slopes. If $\mathcal{L}$ is ample, then it follows from the standard characterization of ample line bundles on toric varieties ([38], Corollary 2.15) that $\varphi_{\mathcal{L}}$ is strictly convex.

Definition 4.16. If $\mathcal{L}$ is ample, we call $\left(X^{\dagger}, \mathcal{L}\right)$ a polarized toric log Calabi-Yau space, and we call the triple

$$
\left(B, \mathscr{P}, \varphi_{\mathcal{L}}\right)
$$

degeneration data associated to $\left(X^{\dagger}, \mathcal{L}\right)$. If $\left(\check{B}, \check{\mathscr{P}}, \check{\varphi}_{\mathcal{L}}\right)$ is the discrete Legendre transform of $\left(B, \mathscr{P}, \varphi_{\mathcal{L}}\right)$, then we call $\check{B}, \check{\mathscr{P}}$ the intersection complex of the polarized toric log Calabi-Yau space $\left(X^{\dagger}, \mathcal{L}\right)$.

See [19], Section 4, for additional discussion of the intersection complex.

4.3. Positive log structures. As we know from Corollary 3.29, it is rare that there is no singular set $Z \subseteq X^{\dagger}$. However, we need to provide some control over these singularities. The following is a useful restriction.

Definition 4.17. Let $X^{\dagger}$ be a toric log Calabi-Yau space with singular set $Z$, with $X \cong X_{0}(B, \mathscr{P}, s)$. Then the log smooth structure $X^{\dagger} \backslash Z \rightarrow$ Spec $k^{\dagger}$ is induced by a section $f \in \Gamma\left(X_{0}(B, \mathscr{P}, s) \backslash\right.$ $\left.Z, \mathcal{L S}_{X_{0}(B, \mathscr{P}, s)}\right)$. We say that $X^{\dagger}$ is positive if the corresponding sections $f_{\omega} \in \Gamma\left(X_{\omega} \backslash q_{\omega}^{-1}(Z), \mathcal{N}_{\omega}^{\times}\right)$(see Theorem 3.28) for $\operatorname{dim} \omega=1$ extend to sections of $\mathcal{N}_{\omega}$ on $X_{\omega}$, i.e., if they have zeros but no poles.

Remark 4.18. It is easy to see that this notion of positivity is independent of the choice of orientation of edges of $\mathscr{P}$.

Proposition 4.19. Let $(B, \mathscr{P})$ be positive, and let $\mathcal{N}_{\omega}$ be the line bundle on $X_{\omega}$ for $\operatorname{dim} \omega=1$ given by Theorem 3.28. Then $\mathcal{N}_{\omega}$ is generated by global sections. In particular, it has a section whose zero-locus does not contain a toric stratum of $X_{\omega}$. Conversely, if $X^{\dagger}$ is positive, then its dual intersection complex $(B, \mathscr{P})$ is positive. 
Proof. By Theorem 3.28, $\mathcal{N}_{X_{\omega}}$ corresponds to the piecewise linear function $\psi_{\omega}$ of Remark 1.56. If $(B, \mathscr{P})$ is positive, then $\psi_{\omega}$ is convex and hence by [38], Corollary $2.15, \mathcal{N}_{X_{\omega}}$ is generated by global sections. Conversely, if $X^{\dagger}$ is positive, there exist sections $f_{\omega} \in \Gamma\left(X_{\omega}, \mathcal{N}_{\omega}\right)$ vanishing only on $q_{\omega}^{-1}(Z)$, hence they do not vanish on any toric stratum by the assumptions on $Z$. Since the base locus of a complete toric linear system is a union of toric strata, $\mathcal{N}_{\omega}$ is globally generated, hence $\psi_{\omega}$ is convex, and $(B, \mathscr{P})$ is positive.

q.e.d.

Proposition 4.20. Let $f: \mathcal{X} \rightarrow \mathcal{S}$ be a toric degeneration. Then $\mathcal{X}_{0}^{\dagger}$ is positive.

Proof. Let $(B, \mathscr{P})$ be the dual intersection complex, and $\omega \in \mathscr{P}$ a one-dimensional face, corresponding to a stratum $S_{\omega}$ of dimension $n-1$. It suffices to check the absence of poles of $f_{\omega} \in \Gamma\left(q_{\omega_{*}} \mathcal{N}_{\omega}\right)$ in codimension one on $S_{\omega}$. Without loss of generality, we can view $\Lambda_{\omega} \cong$ $\mathbb{Z}=M^{\prime} \subseteq M$ with $\omega \subseteq \mathbb{R}$ an interval with endpoints 0 and $l>0$. Choose $d_{\omega}=1$. Then $Q^{\prime}:=C^{\prime}(\omega)^{\vee} \cap\left(N^{\prime} \oplus \mathbb{Z}\right)$ is generated by $p_{1}=(1,0)$, $p_{2}=(-1, l)$ and $\rho=(0,1)$, with relation $p_{1}+p_{2}=l \rho$. Abstractly, $V(\omega) \cong(\operatorname{Spec} k[u, v] /(u v)) \times \mathbb{G}_{m}^{n-1}$, and the log structure on $V(\omega) \backslash Z$ is of the same type as that given by the chart

$$
p_{1} \mapsto u, p_{2} \mapsto v, \rho \mapsto 0 .
$$

If on some open subset $U$ of $V(\omega)$, we have a chart given by

$$
p_{1} \mapsto h_{u} u, p_{2} \mapsto h_{v} v, \rho \mapsto 0
$$

with $h_{u}, h_{v}$ invertible functions as usual, then by the construction of the map $\xi$ in the proof of Theorem 3.22, the corresponding section of $\mathcal{L S}_{V(\omega)}=\mathcal{O}_{V(\omega)}^{\times}$is $\left(h_{u} h_{v}\right)^{-1}$. For the log structure induced by the embedding $\mathcal{X}_{0} \subset \mathcal{X}$ it can be computed as follows. Let $\tilde{u}, \tilde{v} \in \mathcal{O}_{\mathcal{X}, \bar{x}}$ be extensions of $u, v$ at some geometric point $\bar{x}$ in the codimension one stratum of $V(\omega) \backslash Z$. Then $\tilde{u} \tilde{v}$ restricts to zero on the central fibre and hence $\tilde{u} \tilde{v}=t^{l} \cdot h$ for $t$ a generator of the maximal ideal of $\Gamma\left(\mathcal{O}_{\mathcal{S}}\right)$ and $h \in \mathcal{O}_{\mathcal{X}, \bar{x}}^{\times}$. The exponent of $t$ is $l$ because $\mathcal{M}_{\mathcal{X}, \bar{x}} \cong Q^{\prime}$. We may then put $h_{u}=1, h_{v}=\left.h^{-1}\right|_{\mathcal{X}_{0}}$. Thus $f_{\omega}$ is represented by $\left.h\right|_{\mathcal{X}_{0}}$.

Now let $\bar{y}$ be a geometric point of $V(\omega) \cap Z$ and $\tilde{u}, \tilde{v} \in \mathcal{O}_{\mathcal{X}, \bar{y}}$ as before. By what we just said $t^{l}$ divides $\tilde{u} \tilde{v}$ in codimension one and hence everywhere since $\mathcal{X}$ is normal. The quotient $h:=\tilde{u} \tilde{v} / t^{l} \in \mathcal{O}_{\mathcal{X}, \bar{y}}$ is the desired regular extension of $f_{\omega}$ over $\bar{y}$.

q.e.d.

Definition 4.21. For $\sigma \subseteq M_{\mathbb{R}}$ a lattice polytope, we denote by $\mathcal{L S}_{\text {pre }, V(\sigma)}^{+}$the sheaf

$$
\bigoplus_{\substack{\omega \subseteq \sigma \\ \operatorname{dim} \omega=1}} \mathcal{O}_{V_{\omega}}
$$


where $V_{\omega}:=V_{\omega \rightarrow \sigma}$ denotes the codimension one stratum of $V(\sigma)$ corresponding to $\omega$. For $(B, \mathscr{P})$ and open gluing data $s, X=X_{0}(B, \mathscr{P}, s)$, we denote by $\mathcal{L S}_{\text {pre, } X}^{+}$the sheaf on $X$ obtained by gluing the sheaves $\mathcal{L S}_{\text {pre }, V(\sigma)}^{+}$for $\sigma \in \mathscr{P}_{\max }$ using the gluing formula of Theorem 3.27, so that in particular $\mathcal{L} \mathcal{S}_{X}$ is a subsheaf (of sets) of $\mathcal{L S}_{\text {pre, } X}^{+}$.

Note that to specify a positive log Calabi-Yau structure on $X=$ $X_{0}(B, \mathscr{P}, s)$, we need to give a section $f \in \Gamma\left(X, \mathcal{L S}_{\text {pre }, X}^{+}\right)$satisfying: (1) $f$ satisfies the multiplicative conditions given in Theorem 3.22 and (2) the components of $f$ on codimension one strata have no zero sets containing any toric stratum. Now $\mathcal{L S}_{\text {pre, } X}^{+}$is a coherent sheaf on $X$, so if $B$ is compact, $\mathcal{L S}_{\text {pre, } X}^{+}$has a finite dimensional space of sections. Then condition (2) determines some open subset of this space of sections, while the multiplicative condition (1) is a more subtle, closed condition. This allows us to identify the moduli space of positive log Calabi-Yau structures on $X$ as a subvariety of an affine space. We shall examine this more closely in $\S 4.4$.

Remark 4.22. We can now determine the discriminant locus of a dual intersection complex $B$ more precisely in the positive case. Initially, we take $\Delta^{\prime} \subseteq B$ as in Construction 1.26 to be the union of all simplices of $\operatorname{Bar}(\mathscr{P})$ not containing a vertex of $\mathscr{P}$ or the barycenter of a maximal cell. Using Proposition 1.27, we can identify a smaller discriminant locus $\Delta \subseteq \Delta^{\prime}$ as follows if $X^{\dagger}$ is positive and the singular set $Z \subseteq X$ is taken to be minimal.

For any $\sigma \in \operatorname{Bar}(\mathscr{P}), \sigma \subseteq \Delta^{\prime}, \operatorname{dim} \sigma=n-2$, there exist unique $\sigma_{1}, \sigma_{n-1} \in \mathscr{P}$ with $\operatorname{dim} \sigma_{i}=i$ and $\sigma$ containing the barycenters of $\sigma_{1}$ and $\sigma_{n-1}$. In addition, there exists a unique morphism $e_{\sigma}: \sigma_{1} \rightarrow \sigma_{n-1}$ corresponding to the edge of $\sigma$ joining these two barycenters. Furthermore, there exist only two distinct morphisms $f_{i}: \sigma_{n-1} \rightarrow \tau_{i}, i=1,2$, with $\tau_{i} \in \mathscr{P}_{\max }$. Let $e_{i}=f_{i} \circ e_{\sigma}$.

The monodromy around $\sigma$ is now given by $T_{\sigma_{1}}^{e_{1} e_{2}}$ of $\S 1.5$, and hence is trivial if and only if $n_{\sigma_{1}}^{e_{1} e_{2}}=0$.

On the other hand, consider the embedding $F\left(e_{\sigma}\right): \mathbb{P}^{1} \cong X_{\sigma_{n-1}} \rightarrow$ $X_{\sigma_{1}}$. Then by Theorem 3.28 and [38], Corollary 2.15, the line bundle $F\left(e_{\sigma}\right)^{*}\left(\mathcal{N}_{X_{\sigma_{1}}}\right)$ is trivial if and only if $n_{\sigma_{1}}^{e_{1} e_{2}}=0$, in which case the affine structure on $B$ will extend across $\sigma$ by Proposition 1.27. Now the positive log Calabi-Yau structure on $X$ determines a section $f_{\sigma_{1}} \in \Gamma\left(X_{\sigma_{1}}, \mathcal{N}_{X_{\sigma_{1}}}\right)$ which does not vanish on an entire toric stratum of $X_{\sigma_{1}}$. Thus $F\left(e_{\sigma}\right)^{*}\left(\mathcal{N}_{X_{\sigma_{1}}}\right) \cong \mathcal{O}_{X_{\sigma_{n-1}}}$ if and only if $f_{\sigma_{1}}$ does not vanish at any point of $F\left(e_{\sigma}\right)\left(X_{\sigma_{n-1}}\right)$. However, the zero locus of $f_{\sigma_{1}}$ is

$$
\operatorname{cl}\left(q_{\sigma_{1}}^{-1}(Z) \backslash \partial X_{\sigma_{1}}\right) \subseteq X_{\sigma_{1}}
$$


Thus we see that $\Delta$ is the union of all $\sigma \in \operatorname{Bar}(\mathscr{P}), \sigma \subseteq \Delta^{\prime}, \operatorname{codim} \sigma=2$ satisfying

$$
\operatorname{cl}\left(q_{\sigma_{1}}^{-1}(Z) \backslash \partial X_{\sigma_{1}}\right) \cap F\left(e_{\sigma}\right)\left(X_{\sigma_{n-1}}\right) \neq \emptyset .
$$

This gives the precise relationship between $\Delta$ and $Z$.

4.4. Normalized gluing data and examples. We saw in Proposition 2.32 that if $s$ and $s^{\prime}$ differ by an element of $B^{1}\left(\mathscr{P}, \mathcal{Q} \mathscr{P} \otimes \mathbb{G}_{m}(k)\right)$, then $X_{0}(B, \mathscr{P}, s) \cong X_{0}\left(B, \mathscr{P}, s^{\prime}\right)$. We would now like to use open gluing data to parametrize log structures as well as the underlying schemes, so that we can determine explicitly the moduli space of toric log CalabiYau spaces with a given dual intersection complex $(B, \mathscr{P})$.

We begin with the following definition:

Definition 4.23. Let $\sigma \subseteq M_{\mathbb{R}}$ be an $n$-dimensional integral polytope. Let $x \in V(\sigma)$ be the unique zero-dimensional torus orbit. A section $f$ of $\mathcal{L S}_{V(\sigma)}$ (or $\mathcal{L S}_{\text {pre }, V(\sigma)}^{+}$) defined in a neighbourhood of $x$ is said to be normalized if, viewing

$$
f=\left(f_{\omega}\right) \in \bigoplus_{\substack{\omega \subseteq \sigma \\ \operatorname{dim} \omega=1}} \mathcal{O}_{V_{\omega}}
$$

as in Theorem 3.22, $f_{\omega}$ takes the value 1 at $x$ for each $\omega \subseteq \sigma, \operatorname{dim} \omega=1$.

Note that this condition can be rephrased in terms of a chart for the log structure near the point $x$. Given $X=V(\sigma)$ a scheme with the étale topology, and a chart

$$
\alpha: P_{\sigma} \longrightarrow \mathcal{O}_{X, \bar{x}}
$$

of the form $p \mapsto h_{p} z^{p}$ as usual, with $h_{p}$ a germ of an invertible function at $x \in X(p)=\operatorname{cl}\left\{z^{p} \neq 0\right\}$, we obtain a map

$$
\phi: \partial P_{\sigma} \longrightarrow k^{\times}
$$

given by $p \mapsto h_{p} \bmod \mathbf{m}_{\overline{\mathbf{x}}}$.

This map is piecewise multiplicative, and it is not difficult to see that the corresponding section $f$ of $\mathcal{L S}_{V(\sigma)}$ is normalized if and only if this map is in fact multiplicative on $N$.

In particular, even if $f$ is not normalized, $\phi$ induces an automorphism of $V(\sigma)$ via $z^{p} \mapsto \phi(p) z^{p}$, and it is then clear that the pull-back of the chart $\alpha$ by $\phi^{-1}$ yields a section of $\mathcal{L S}_{V(\sigma)}$ in a neighbourhood of $x \in V(\sigma)$ which is normalized.

Definition 4.24. Let $s$ be open gluing data for $(B, \mathscr{P})$, with canonical projection maps $p_{\sigma}: V(\sigma) \rightarrow X_{0}(B, \mathscr{P}, s)$ for each $\sigma \in \mathscr{P}_{\max }$. Then a section $f \in \Gamma\left(X, \mathcal{L S}_{\text {pre }, X}^{+}\right)$is normalized if $p_{\sigma}^{-1}(f)$ is a normalized section of $\mathcal{L S}_{\text {pre }, X}^{+}$for all $\sigma \in \mathscr{P}_{\text {max }}$. If in fact $f$ determines a log Calabi-Yau structure $X^{\dagger}$ on $X$, i.e., $f \in \Gamma\left(X \backslash Z, \mathcal{L S}_{X}\right)$ for some singular set $Z \subseteq X$ not containing any toric stratum of $X$, and in addition $f$ is normalized, then we say $X^{\dagger}$ is normalized by the open gluing data $s$. 
By the above discussion, there is always some open gluing data with respect to which a toric log Calabi-Yau space $X^{\dagger}$ is normalized. Conversely we have the following criterion.

Proposition 4.25. Let $(B, \mathscr{P})$ be positive, and let $s \in Z^{1}\left(\mathscr{P}, \mathcal{Q}_{\mathscr{P}} \otimes\right.$ $\left.\mathbb{G}_{m}\right)$ be open gluing data, $X=X_{0}(B, \mathscr{P}, s)$. Then there exists a normalized section $f \in \Gamma\left(X, \mathcal{L S}_{\mathrm{pre}, X}^{+}\right)$if and only if the following condition holds:

Condition (LC): Let $\omega \in \mathscr{P}$ be a one-dimensional cell, $\sigma_{1}, \sigma_{2} \in$ $\mathscr{P}_{\max }, \tau \in \mathscr{P}$ with a diagram

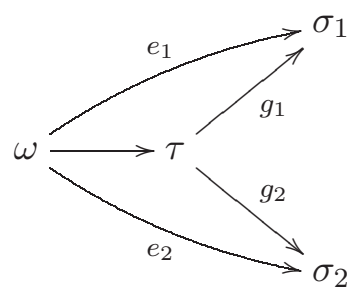

Then if $T_{\omega}^{e_{1} e_{2}}=0$ we must have $\mathrm{D}\left(s_{g_{1}}, \omega, \sigma_{1}\right)=\mathrm{D}\left(s_{g_{2}}, \omega, \sigma_{2}\right)$.

Proof. Suppose there exists a normalized $f \in \Gamma\left(X, \mathcal{L S}_{\text {pre }, X}^{+}\right)$. This is induced by sections $f_{\sigma}=p_{\sigma}^{-1}(f) \in \Gamma\left(V(\sigma), \mathcal{L S}_{\text {pre }, V(\sigma)}^{+}\right), \sigma \in \mathscr{P}_{\max }$, which glue as given in Theorem 3.27. Now

$$
\mathcal{L S}_{\text {pre, } V(\sigma)}^{+}=\bigoplus_{\substack{e \in \bigsqcup_{\operatorname{dim} \omega=1}(\omega, \sigma) \\ \operatorname{dim} \omega=1}} \mathcal{O}_{V_{e}},
$$

so $f_{\sigma}$ decomposes into components $f_{\sigma, e} \in \Gamma\left(\mathcal{O}_{V_{e}}\right)$. We can write

$$
f_{\sigma, e}=\sum_{p \in P_{e}} f_{\sigma, e, p} z^{p}
$$

where $P_{e}$ is the face of $P_{\sigma}$ corresponding to $e$, with $V_{e}=\operatorname{Spec} k\left[P_{e}\right]$. The normalization condition says that $f_{\sigma, e, 0}=1$. Given any diagram (4.2) such that $\left(g_{1}, g_{2}\right)$ is a maximal pair, Theorem 3.27 tells us that if $n_{\omega}^{e_{1} e_{2}}=0$, then

$$
\left.f_{\sigma_{2}, e_{2}}\right|_{V\left(\tilde{\tau}_{2}\right)} \frac{\mathrm{D}\left(s_{g_{1}}, \omega, \sigma_{1}\right)}{\mathrm{D}\left(s_{g_{2}}, \omega, \sigma_{2}\right)}=\Phi_{g_{1} g_{2}}(s)^{*}\left(\left.f_{\sigma_{1}, e_{1}}\right|_{V\left(\tilde{\tau}_{1}\right)}\right) .
$$

Comparing constant coefficients gives

$$
f_{\sigma_{2}, e_{2}, 0} \cdot \frac{\mathrm{D}\left(s_{g_{1}}, \omega, \sigma_{1}\right)}{\mathrm{D}\left(s_{g_{2}}, \omega, \sigma_{2}\right)}=f_{\sigma_{1}, e_{1}, 0} .
$$

But $f_{\sigma, e, 0}=1$ for all $\sigma, e$ by the normalization assumption, so $\mathrm{D}\left(s_{g_{1}}, \omega\right.$, $\left.\sigma_{1}\right)=\mathrm{D}\left(s_{g_{2}}, \omega, \sigma_{2}\right)$ whenever $n_{\omega}^{e_{1} e_{2}}=0$. This is the required condition when $\left(g_{1}, g_{2}\right)$ is maximal. 
Now if $\left(g_{1}, g_{2}\right)$ is not maximal, then there is a maximal pair $\left(h_{1}, h_{2}\right)$ with $\left(g_{1}, g_{2}\right)<\left(h_{1}, h_{2}\right)$, i.e., there exists $f: \tau \rightarrow \rho, h_{i}: \rho \rightarrow \sigma_{i}$ with $g_{i}=h_{i} \circ f$. Then

$$
\begin{aligned}
\mathrm{D}\left(s_{g_{i}}, \omega, \sigma_{i}\right) & =\mathrm{D}\left(\left.s_{h_{i}}\right|_{\tau} \cdot s_{f}, \omega, \sigma_{i}\right) \\
& =\mathrm{D}\left(\left.s_{h_{i}}\right|_{\tau}, \omega, \sigma_{i}\right) \mathrm{D}\left(s_{f}, \omega, \sigma_{i}\right) \\
& =\mathrm{D}\left(s_{h_{i}}, \omega, \sigma_{i}\right) \mathrm{D}\left(s_{f}, \omega, \sigma_{i}\right) .
\end{aligned}
$$

Now $\mathrm{D}\left(s_{h_{1}}, \omega, \sigma_{1}\right)=\mathrm{D}\left(s_{h_{2}}, \omega, \sigma_{2}\right)$ since $\left(h_{1}, h_{2}\right)$ is maximal and $n_{\omega}^{e_{1}, e_{2}}=$ 0 , while $\mathrm{D}\left(s_{f}, \omega, \sigma_{1}\right)=\mathrm{D}\left(s_{f}, \omega, \sigma_{2}\right)$ by Remark 3.26 and $n_{\omega}^{e_{1} e_{2}}=0$. Thus Condition (LC) holds.

Conversely, we will show how to produce a normalized section $f$ of $\mathcal{L S}_{\text {pre }, X}^{+}$by constructing $f_{\sigma} \in \Gamma\left(V(\sigma), \mathcal{L S}_{\text {pre, } X}^{+}\right)$which glue correctly. To do so, first fix for every $\omega \in \mathscr{P}$ with $\operatorname{dim} \omega=1$ a $\sigma_{\omega} \in \mathscr{P}_{\max }$ with a morphism $e_{\omega}: \omega \rightarrow \sigma_{\omega}$. Let $y \in \omega$ be a point near $v_{\omega}^{+}$, and let $\check{\Delta}(\omega)$ be, as in Definition 1.58, the convex hull in $\Lambda_{\omega, \mathbb{R}}^{\perp} \subseteq \check{\Lambda}_{\mathbb{R}, y}$ of the set

$$
\left\{n_{\omega}^{e_{\omega} e^{\prime}} \mid e^{\prime}: \omega \rightarrow \sigma^{\prime} \in \mathscr{P}_{\max }\right\} \subseteq \Lambda_{\omega}^{\perp} \subseteq \check{\Lambda}_{y} .
$$

This is the Newton polytope of the line bundle $\mathcal{N}_{\omega}$ on $X_{\omega}$, up to translation. In particular, any

$$
f_{\sigma_{\omega}, e_{\omega}}=\sum_{p \in \check{\Delta}(\omega) \cap \check{\Lambda}_{y}} f_{\sigma_{\omega}, e_{\omega}, p} z^{p}
$$

gives a well-defined regular section of $\mathcal{N}_{\omega}$ on $X_{\omega}$. Explicitly, given a diagram

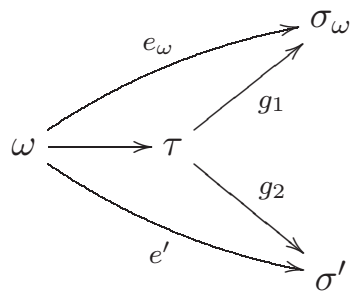

with $\left(g_{1}, g_{2}\right)$ maximal, the above choice for $f_{\sigma_{\omega}, e_{\omega}}$ determines $f_{\sigma^{\prime}, e^{\prime}}$ via the formula (coming from Theorem 3.27)

$$
\begin{aligned}
f_{\sigma^{\prime}, e^{\prime}}= & z^{-n_{\omega}^{e_{\omega} e^{\prime}}} \frac{\mathrm{D}\left(s_{g_{2}}, \omega, \sigma^{\prime}\right)}{\mathrm{D}\left(s_{g_{1}}, \omega, \sigma_{\omega}\right)} s_{g_{2}}\left(n_{\omega}^{e_{\omega} e^{\prime}}\right)^{-1} \Phi_{g_{1} g_{2}}(s)^{*}\left(f_{\sigma_{\omega}, e_{\omega}}\right) \\
= & \frac{\mathrm{D}\left(s_{g_{2}}, \omega, \sigma^{\prime}\right)}{\mathrm{D}\left(s_{g_{1}}, \omega, \sigma_{\omega}\right)} s_{g_{2}}\left(n_{\omega}^{e} e^{\prime}\right)^{-1} \\
& \cdot \sum_{p \in \check{\Delta}(\omega) \cap \check{\Lambda}_{y}} f_{\sigma_{\omega}, e_{\omega}, p} \frac{\Phi_{g_{1} g_{2}}(s)^{*}\left(z^{p}\right)}{z^{p}} z^{p-n_{\omega}^{e_{\omega} e^{\prime}}} .
\end{aligned}
$$

Here on $X_{\omega}, \Phi_{g_{1} g_{2}}(s)^{*}\left(z^{p}\right) / z^{p}$ is just an element of $k^{\times}$. Now we require that the section be normalized, i.e., the constant term of each $f_{\sigma, e}$ be 1 . 
This implies that for each diagram (4.3), we must have

$$
\begin{aligned}
1 & =\frac{\mathrm{D}\left(s_{g_{2}}, \omega, \sigma^{\prime}\right)}{\mathrm{D}\left(s_{g_{1}}, \omega, \sigma_{\omega}\right)} s_{g_{2}}\left(n_{\omega}^{e_{\omega} e^{\prime}}\right)^{-1} f_{\sigma_{\omega}, e_{\omega}, n_{\omega}^{e \omega e^{\prime}}} \frac{\Phi_{g_{1} g_{2}}(s)^{*}\left(z^{n_{\omega}^{e \omega e^{\prime}}}\right)}{z^{n_{\omega}^{e} e^{\prime}}} \\
& =\frac{\mathrm{D}\left(s_{g_{2}}, \omega, \sigma^{\prime}\right)}{\mathrm{D}\left(s_{g_{1}}, \omega, \sigma_{\omega}\right)} s_{g_{1}}\left(n_{\omega}^{e_{\omega} e^{\prime}}\right)^{-1} f_{\sigma_{\omega}, e_{\omega}, n_{\omega}^{e} e^{\prime}}
\end{aligned}
$$

using $\Phi_{g_{1} g_{2}}(s)^{*}\left(z^{n_{\omega}^{e} e^{\prime}}\right)=s_{g_{1}}\left(n_{\omega}^{e_{\omega} e^{\prime}}\right)^{-1} z^{n_{\omega}^{e_{\omega} e^{\prime}}} s_{g_{2}}\left(n_{\omega}^{e_{\omega} e^{\prime}}\right)$. Thus

$$
f_{\sigma_{\omega}, e_{\omega}, n_{\omega}^{e} e^{\prime}}=\frac{\mathrm{D}\left(s_{g_{1}}, \omega, \sigma_{\omega}\right)}{\mathrm{D}\left(s_{g_{2}}, \omega, \sigma^{\prime}\right)} s_{g_{1}}\left(n_{\omega}^{e_{\omega} e^{\prime}}\right) .
$$

Thus the coefficients

$$
\left\{f_{\sigma_{\omega}, e_{\omega}, n_{\omega}^{e} e^{e^{\prime}}} \mid e^{\prime}: \omega \rightarrow \sigma^{\prime} \in \mathscr{P}_{\max }\right\}
$$

of $f_{\sigma_{\omega}, e_{\omega}}$ are completely determined by the normalization condition, while all other coefficients $f_{\sigma_{\omega}, e_{\omega}, p}$ may be chosen at will.

There is one point that we must check here, which is that if $n_{\omega}^{e_{\omega} e^{\prime}}=$ $n_{\omega}^{e_{\omega} e^{\prime \prime}}$, then the values of $f_{\sigma_{\omega}, e_{\omega}, n_{\omega}^{e} e^{\prime}}$ and $f_{\sigma_{\omega}, e_{\omega}, n_{\omega}^{e} e^{\prime \prime}}$ given by formula (4.4) coincide. Since $n_{\omega}^{e^{\prime} e^{\prime \prime}}=n_{\omega}^{e_{\omega} e^{\prime \prime}}-n_{\omega}^{e_{\omega} e^{\prime}}$, it is enough to check that if $n_{\omega}^{e_{\omega} e^{\prime}}=0$, then (4.4) gives $f_{\sigma_{\omega}, e_{\omega}, n_{\omega}^{e} e^{\prime}}=1$. But this follows immediately from Condition (LC).

q.e.d.

Theorem 4.26. Let $(B, \mathscr{P})$ be positive, with either $\Delta=\emptyset$ or $\operatorname{dim} B \leq 2$, and let $s \in Z^{1}\left(\mathscr{P}, \mathcal{Q}_{\mathscr{P}} \otimes \mathbb{G}_{m}\right)$ be open gluing data satisfying Condition (LC) of Proposition 4.25. Then if $X=X_{0}(B, \mathscr{P}, s)$, any normalized $f \in \Gamma\left(X, \mathcal{L S}_{\mathrm{pre}, X}^{+}\right)$determines a positive normalized log Calabi-Yau structure on $X$.

Proof. Write $f=\left(f_{\sigma, e}\right)$ with $f_{\sigma, e} \in \Gamma\left(V_{e}, \mathcal{O}_{V_{e}}\right)$ for any $e: \omega \rightarrow \sigma \in$ $\mathscr{P}_{\max }, \operatorname{dim} \omega=1$. Because $f_{\sigma, e}$ is 1 at the zero-dimensional stratum of $V_{e}$, the zero set of $f_{\sigma, e}$ cannot contain any toric stratum of $V_{e}$. Thus if

$$
Z=\bigcup_{\sigma, e} q_{\sigma}\left(\left\{f_{\sigma, e}=0\right\}\right) \subseteq X
$$

then $Z$ does not contain any toric stratum of $X$.

If $\Delta=\emptyset$, then in fact $Z=\emptyset$ anyway, as $f_{\sigma, e}$ is constant, hence identically 1 . Then the multiplicative condition of Theorem 3.22 is satisfied automatically. If $\Delta \neq \emptyset$, then $\operatorname{dim} B=2$, and Theorem 3.22 imposes a condition for each two-dimensional, i.e., maximal, cell $\sigma$. But $X_{\sigma}$ is just a point, and the formula of Theorem 3.22 imposes a condition on the functions $f_{\sigma, e} \mid X_{\sigma}$. However, since $f_{\sigma, e}$ takes the value 1 at this point by the normalization condition, the multiplicative condition of Theorem 3.22 is automatically satisfied, and $f \in \Gamma\left(X \backslash Z, \mathcal{L S}_{X}\right)$. q.e.d. 
Remark 4.27. In the case $\Delta=\emptyset$, the open gluing data in fact determine the log Calabi-Yau structure uniquely. However, in the twodimensional case, when $\Delta \neq \emptyset$, the open gluing data do not necessarily determine the log Calabi-Yau structure uniquely. Indeed, if $\omega$ contains a singularity of $B$ with holonomy $\left(\begin{array}{ll}1 & n \\ 0 & 1\end{array}\right)$ (as in Example 3.30) then $\mathcal{N}_{\omega} \cong \mathcal{O}_{\mathbb{P}^{1}}(n)$, and $\check{\Delta}(\omega)$ is a line segment of length $n$. The coefficients of the monomials corresponding to the endpoints are determined by (4.4), but the coefficients corresponding to the interior vertices are free to be chosen arbitrarily in $k$. Thus for given open gluing data $s$ satisfying Condition (LC), the set of normalized log Calabi-Yau structures on $X_{0}(B, \mathscr{P}, s)$ is parametrized by a vector space of some dimension. Specifically, if $\Delta=\left\{p_{1}, \ldots, p_{s}\right\}$ with monodromy around $p_{i}$ given by $\left(\begin{array}{cc}1 & n_{i} \\ 0 & 1\end{array}\right)$, then the dimension of the space of positive normalized log Calabi-Yau structures on $X_{0}(B, \mathscr{P}, s)$ is $\sum_{i=1}^{s}\left(n_{i}-1\right)$. In particular, if $n_{i}=1$ for all $i$, then the $\log$ Calabi-Yau structure is uniquely determined by $s$.

Example 4.28. Theorem 4.26 is not true in dimensions three and higher, and it is an important feature of the theory that the space of possible log structures is not even non-singular in general. We will now give a quite general local example, which can be fitted into a global example, featuring two maximal cells as in Construction 2.15. Let $M_{1}=$ $M_{2}=M=\mathbb{Z}^{n}$, and choose some primitive vector $\check{d}_{\rho} \in N$. Let $M^{\prime}=$ $\check{d}_{\rho}^{\perp} \subseteq M$, and let $\rho \subseteq M_{\mathbb{R}}^{\prime}$ be an $n$-1-dimensional lattice polytope. Choose $\sigma_{1} \subseteq M_{1} \otimes \mathbb{R}, \sigma_{2} \subseteq M_{2} \otimes \mathbb{R}$ to be $n$-dimensional lattice polytopes with $\sigma_{i} \cap M_{\mathbb{R}}^{\prime}=\rho$ and such that

$$
\sigma_{1} \subseteq\left\{m \in M_{1} \otimes \mathbb{R} \mid\left\langle\check{d}_{\rho}, m\right\rangle \leq 0\right\}
$$

and

$$
\sigma_{2} \subseteq\left\{m \in M_{2} \otimes \mathbb{R} \mid\left\langle\check{d}_{\rho}, m\right\rangle \geq 0\right\} .
$$

Next, we define a singular affine structure with boundary on $\sigma_{1} \cup \sigma_{2}$ as follows. Embed $M_{1}$ in $M \oplus \mathbb{Z}$ by $m \mapsto(m, 1)$ and $M_{2}$ in $M \oplus \mathbb{Z}$ by $m \mapsto\left(m, 1-\left\langle\check{d}_{\rho}, m\right\rangle\right)$. Thus we obtain $\sigma_{1} \cup \sigma_{2} \subseteq M_{\mathbb{R}} \oplus \mathbb{R}$. To define the desired affine structure, we have the canonical embeddings $\operatorname{Int}\left(\sigma_{i}\right) \subseteq\left(M_{i}\right)_{\mathbb{R}}$, giving affine coordinates on $\operatorname{Int}\left(\sigma_{i}\right)$. In a neighbourhood $U_{v}$ of each vertex $v \in M^{\prime}$ of $\rho,(v, 1) \in M \oplus \mathbb{Z}$, we obtain a chart $U_{v} \rightarrow\left(M_{\mathbb{R}} \oplus \mathbb{R}\right) / \mathbb{R}(v, 1)$ simply by projection. It is easy to see this is a homeomorphism onto its image. Take $\Delta \subseteq \rho$ to be the union of codimension one simplices of the first barycentric subdivision of $\rho$ not containing vertices of $\rho$. We can then choose open sets $U_{v}$ so that $\left\{\operatorname{Int}\left(\sigma_{1}\right), \operatorname{Int}\left(\sigma_{2}\right)\right\} \cup\left\{U_{v}\right\}$ form an open covering of $\left(\sigma_{1} \cup \sigma_{2}\right) \backslash \Delta$ and $U_{v} \cap U_{w}=\emptyset$ if $v \neq w$. It follows that the affine coordinate charts 
defined on these sets define an integral affine structure. Parallel transport $\psi_{v}: M_{2} \rightarrow M_{1}$ through (or near) a vertex $v$ of $\rho$ is given by the composition

$$
M_{2} \hookrightarrow M \oplus \mathbb{Z} \longrightarrow(M \oplus \mathbb{Z}) / \mathbb{Z}(v, 1) \longrightarrow M_{1},
$$

where the second map is projection and the third is the inverse of the composed map

$$
M_{1} \hookrightarrow M \oplus \mathbb{Z} \longrightarrow(M \oplus \mathbb{Z}) / \mathbb{Z}(v, 1)
$$

Thus $\psi_{v}$ is the composition

$$
\begin{aligned}
M_{2} \ni m & \mapsto\left(m, 1-\left\langle\check{d}_{\rho}, m\right\rangle\right) \\
& \mapsto\left(m, 1-\left\langle\check{d}_{\rho}, m\right\rangle\right) \quad \bmod (v, 1) \\
& =\left(m+\left\langle\check{d}_{\rho}, m\right\rangle v, 1\right) \quad \bmod (v, 1) \\
& \mapsto m+\left\langle\check{d}_{\rho}, m\right\rangle v \in M_{1} .
\end{aligned}
$$

Thus if $\omega$ is an edge of $\rho, d_{\omega}$ a generator of $\Lambda_{\omega}$, with $v_{\omega}^{-}-v_{\omega}^{+}=p d_{\omega}$ for some positive integer $p$, then

$$
\begin{aligned}
T_{\omega \rightarrow \rho}(m) & =\psi_{v_{\omega}^{-}} \circ \psi_{v_{\omega}^{+}}^{-1}(m) \\
& =m+\left\langle p \check{d}_{\rho}, m\right\rangle d_{\omega},
\end{aligned}
$$

so $n_{\omega \rightarrow \rho}=p \check{d}_{\rho}$.

We will now restrict to the case that $p=1$ for all edges $\omega$ of $\rho$, for ease of the discussion, and to make contact with the work of Altmann [1]. We now have as usual, as in Construction 2.15, $\rho=\rho_{1} \subseteq \sigma_{1}$, $\rho=\rho_{2} \subseteq \sigma_{2}, V\left(\rho_{i}\right) \subseteq V\left(\sigma_{i}\right)$, and after a choice of $s_{1}$ and $s_{2}$, an isomorphism $\Phi_{\sigma_{1} \sigma_{2}}(s): V\left(\rho_{2}\right) \rightarrow V\left(\rho_{1}\right)$. Gluing along this isomorphism gives $X$.

Note that $X$ contains a one-dimensional stratum $X_{\rho} \cong \mathbb{P}^{1}$, and for each edge $\omega$ of $\rho$, we have the codimension 1 stratum $X_{\omega}$ containing $X_{\rho}$. In addition, we have the line bundle $\mathcal{N}_{\omega}$ on $X_{\omega}$, and the value of $n_{\omega \rightarrow \rho}=\check{d}_{\rho}$ tells us that $\left.\mathcal{N}_{\omega}\right|_{X_{\rho}}=\mathcal{O}_{\mathbb{P}^{1}}(1)$. Thus a normalized section $f \in \Gamma\left(X, \mathcal{L S}_{\mathrm{pre}, X}^{+}\right)$must satisfy

$$
\left.f_{\sigma_{1}, \omega \rightarrow \sigma_{1}}\right|_{X_{\rho}}=1+c_{\omega} x
$$

where $x=z^{\check{d}_{\rho}}$. Here $c_{\omega}$ is completely determined by $s_{1}$ and $s_{2}$ by formula (4.4). We note that the choice of $s_{1}$ and $s_{2}$ may not lead to an arbitrary choice of the $c_{\omega}$ 's. In addition, if $f$ is to determine a log CalabiYau structure, we need the multiplicative condition of Theorem 3.22 to hold. In other words, for every two-dimensional cell $\eta \subseteq \rho$, we have

$$
\left.\prod_{\substack{\omega \subseteq \eta \\ \operatorname{dim} \omega=1}} d_{\omega} \otimes f_{\sigma_{1}, \omega \rightarrow \sigma_{1}}^{\epsilon_{\eta}(\omega)}\right|_{X_{\eta}}=1 .
$$


Restricting further to $X_{\rho}$ gives the relation

$$
\prod_{\substack{\omega \subseteq \eta \\ \operatorname{dim} \omega=1}} d_{\omega} \otimes\left(1+c_{\omega} x\right)^{\epsilon_{\eta}(\omega)}=1 .
$$

Let us carry this out explicitly for the following $\rho \subseteq M_{\mathbb{R}}^{\prime}$, with orientations as depicted:

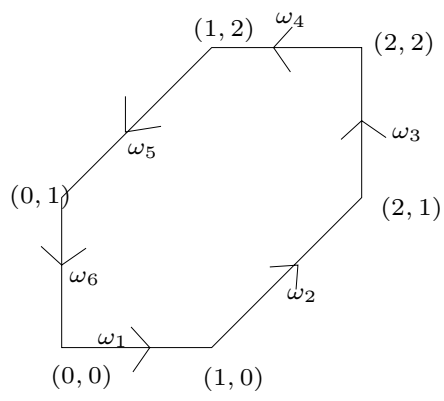

Then we obtain in $M^{\prime}$

$$
d_{\omega_{i}}=(1,0),(1,1),(0,1),(-1,0),(-1,-1),(0,-1) \text { for } i=1, \ldots, 6 .
$$

Splitting (4.5) into its two components, we get the equations (writing $\left.c_{i}=c_{\omega_{i}}\right)$

$$
\begin{aligned}
& \left(1+c_{1} x\right)\left(1+c_{2} x\right)=\left(1+c_{4} x\right)\left(1+c_{5} x\right) \\
& \left(1+c_{2} x\right)\left(1+c_{3} x\right)=\left(1+c_{5} x\right)\left(1+c_{6} x\right) .
\end{aligned}
$$

Comparing coefficients of powers of $x$, we get

$$
\begin{aligned}
c_{1}+c_{2} & =c_{4}+c_{5} \\
c_{2}+c_{3} & =c_{5}+c_{6} \\
c_{1} c_{2} & =c_{4} c_{5} \\
c_{2} c_{3} & =c_{5} c_{6} .
\end{aligned}
$$

This defines a three-dimensional scheme $S$ in $\mathbb{G}_{m}^{6}$, with irreducible components being a $\mathbb{G}_{m}^{3}$ given by the equations

$$
c_{1}=c_{4}, \quad c_{2}=c_{5} \quad c_{3}=c_{6}
$$

and a $\mathbb{G}_{m}^{2}$ given by the equations

$$
c_{2}=c_{4}=c_{6}, \quad c_{1}=c_{3}=c_{5} .
$$

As we noted earlier, because the $c_{i}$ depend on $s_{1}$ and $s_{2}$, the $c_{i}$ cannot be chosen independently, but it is easy to see that the only restriction on the $c_{i}$ is that

$$
\prod_{i=1}^{6} d_{\omega_{i}} \otimes c_{i}=(1,1)
$$

but this condition has already been incorporated into the above equations. It can also be checked that multiplying $s_{1}$ or $s_{2}$ by a multiplicative function $N_{i} \rightarrow \mathbb{G}_{m}$ has the effect of replacing $c_{1}, \ldots, c_{6}$ with 
$c^{\prime} c_{1}, \ldots, c^{\prime} c_{6}$. This does not change the isomorphism class of the gluing, and one obtains isomorphic log Calabi-Yau structures from these gluings. Hence the space of all open gluing data modulo this action fibres over $S / \mathbb{G}_{m}$, where the action of $\mathbb{G}_{m}$ on $S$ is the diagonal one, and $S / \mathbb{G}_{m}$ consists of a union of a one-dimensional torus and a two-dimensional torus meeting at a single point.

The equations determining $S / \mathbb{G}_{m}$ are precisely the same ones defining the deformation space of a cone over a del Pezzo surface of degree 6 , as computed in [1]. The hexagon we are using of course is the toric data which defines the del Pezzo surface of degree 6 .

The explanation is as follows. Let $Y$ be the four-dimensional toric variety defined by the fan $\Sigma$ in $M_{\mathbb{R}} \oplus \mathbb{R}$ consisting of the cones over $\sigma_{1}$ and $\sigma_{2}$ and their faces embedded in $M_{\mathbb{R}} \oplus \mathbb{R}$ as above. Then the cone over $\rho$ is a cone in $\Sigma$, corresponding to a one-dimensional stratum $Y_{\rho}$ of $Y$, with $Y_{\rho} \cong \mathbb{P}^{1}$. The singularities of $Y$ along $Y_{\rho}$ are locally isomorphic to a cone over a del Pezzo surface of degree 6 . If we take a general anti-canonical hypersurface $H$ in $Y$, then $H \cap Y_{\rho}$ consists of one point, and $H$ has a del Pezzo cone singularity at that point. However, $H$ can be viewed as a partial smoothing of the anti-canonical hypersurface $H_{0}$ consisting of the union of all toric divisors of $Y$, and this is precisely $X_{0}(B, \mathscr{P}, 1)$, where 1 denotes the trivial gluing data $s_{1}=s_{2}=1$. The point is that we expect the moduli of $\log$ Calabi-Yau structures on $X_{0}(B, \mathscr{P}, 1)$ should be in some ways similar to (but not isomorphic to) the moduli space of $H$, and thus it makes sense to see the same structures appearing in $\operatorname{Def}(H)$ and the moduli of log Calabi-Yau structures on $H_{0}$.

While this example is local, one expects that there are global examples where the space of positive log Calabi-Yau spaces coming from general $(B, \mathscr{P})$ can be singular because of the above considerations.

It is not difficult to prove in three dimensions that $X_{0}(B, \mathscr{P}, 1)$ (trivial gluing data) always carries a log Calabi-Yau structure. However, it is not clear if this is true in higher dimensions, and it is not so useful because to study mirror symmetry, we would like to describe the entire space of positive log Calabi-Yau spaces with dual intersection complex $(B, \mathscr{P})$. Therefore, in the next section, we will restrict to situations where the behaviour of Example 4.28 does not occur.

\section{Example 4.29.}

1) The toric degeneration of Example 4.2, (1) has dual intersection complex $(B, \mathscr{P})$ as defined in Examples 1.18 and 1.23, (2). Details of this case and a much more general case including the BatyrevBorisov construction are treated in $[\mathbf{1 8}]$.

2) The dual intersection complex for the toric degeneration in Example $4.2,(2)$, arising from the example of Aspinwall and Morrison, 
has dual intersection complex as constructed in Examples 1.19 and $1.23,(2)$.

3) Finally, Example 1.20 gives the dual intersection complex of a degeneration of Enriques surfaces obtained by taking a quotient of a family of $\mathrm{K} 3$ surfaces in $\mathbb{P}_{\Xi^{*}}$. We leave the details to the reader.

We end this section with the following observation and definition.

Proposition 4.30. Let $s, s^{\prime}$ be open gluing data for $(B, \mathscr{P})$, and suppose there are log Calabi-Yau structures $X=X_{0}(B, \mathscr{P}, s)^{\dagger}$ and $X^{\prime}=$ $X_{0}\left(B, \mathscr{P}, s^{\prime}\right)^{\dagger}$ normalized by the gluing data $s$ and $s^{\prime}$ respectively, and suppose there is an isomorphism of $\log$ spaces $\varphi: X \rightarrow X^{\prime}$ preserving $B$. Then there exists $t=\left(t_{\sigma}\right)_{\sigma \in \mathscr{P}}, t_{\sigma} \in P M(\sigma)$, such that $s_{e}^{\prime}=\left.t_{\tau}^{-1} s_{e} t_{\sigma}\right|_{\tau}$ for any $e: \tau \rightarrow \sigma$. Furthermore, for $\sigma \in \mathscr{P}_{\max }, t_{\sigma}$ is induced by an element of $\Lambda_{y} \otimes \mathbb{G}_{m}$ for $y \in \operatorname{Int}(\sigma)$. In this case, we say $s$ and $s^{\prime}$ are equivalent open gluing data.

Proof. The only thing new over Proposition 2.32 is the last statement. As in loc. cit. the isomorphism $\varphi$ is induced by a map

$$
\tilde{\varphi}: \coprod_{\sigma \in \mathscr{P}_{\max }} V(\sigma) \longrightarrow \coprod_{\sigma \in \mathscr{P}_{\max }} V(\sigma),
$$

in which each $\tilde{\varphi}_{\sigma}: V(\sigma) \rightarrow V(\sigma)$ is induced by some $t_{\sigma} \in P \check{M}(\sigma)$. However, if $p_{\sigma}: V(\sigma) \rightarrow X, p_{\sigma^{\prime}}: V(\sigma) \rightarrow X^{\prime}$ are the projections, $f \in$ $\Gamma\left(X, \mathcal{L S}_{\mathrm{pre}, X}^{+}\right), f^{\prime} \in \Gamma\left(X^{\prime}, \mathcal{L S}_{\text {pre }, X^{\prime}}^{+}\right)$inducing the log Calabi-Yau structures, then $p_{\sigma}^{-1}(f)$ and $\left(p_{\sigma}^{\prime}\right)^{-1}(f)$ are both normalized, and $\tilde{\varphi}_{\sigma}^{-1} p_{\sigma}^{-1}(f)=$ $\left(p_{\sigma}^{\prime}\right)^{-1}\left(f^{\prime}\right)$. However, as we pointed out in the discussion following Definition 4.21 , the pull-back of a normalized section of $\mathcal{L S}_{\text {pre, } V(\sigma)}^{+}$via $t_{\sigma}$ is normalized if and only if $t_{\sigma}$ is a multiplicative function on $\check{\Sigma}_{\sigma}$ (for $\left.\sigma \in \mathscr{P}_{\max }\right)$ rather than piecewise multiplicative, i.e., comes from an element of $\Lambda_{y} \otimes \mathbb{G}_{m}$ for $y \in \operatorname{Int}(\sigma)$. We then complete the proof as in Proposition 2.32.

q.e.d.

\section{Simplicity and mirror symmetry}

We are now close to our first goal of realising mirror symmetry for toric log Calabi-Yau spaces. The chief problem at this point, however, is that for general positive $(B, \mathscr{P})$, the moduli space of all positive log Calabi-Yau spaces with dual intersection complex $(B, \mathscr{P})$ may be quite complicated. We have already seen in Example 4.28 that this moduli space may well be singular, and that this reflects the fact that a partial smoothing of a log Calabi-Yau space, if it exists, might itself have obstructed deformation theory. On the mirror side this should correspond to singular moduli spaces of complexified Kähler or symplectic structures, and it is unclear what this should mean. Also, for more refined 
versions of mirror symmetry involving Frobenius or $A_{\infty}$-structures it is convenient if not indispensable to deal with smooth moduli spaces. Therefore, we need to restrict attention to a class of log Calabi-Yau spaces which are well-behaved. We do so by restricting to the case that $(B, \mathscr{P})$ is simple $(\S 1.5)$.

Once again, as in $\S 4$, we always work over $S=\operatorname{Spec} k, k$ an algebraically closed field.

5.1. Moduli of toric log Calabi-Yau spaces in the simple case. Consider $e: \tau_{1} \rightarrow \tau_{2}$ and an element $s \in \Gamma\left(W_{e}, i_{*} \Lambda \otimes \mathbb{G}_{m}\right)$. This in fact defines an element $s \in P \breve{M}\left(\tau_{1}\right)$ as follows. Choose any $\tau_{2} \rightarrow \sigma \in \mathscr{P}_{\max }$. Then $\operatorname{Int}(\sigma) \cap W_{e}$ is non-empty, and for any point $z \in \operatorname{Int}(\sigma) \cap W_{e}, s$ yields a germ $s_{z} \in \Lambda_{z} \otimes \mathbb{G}_{m}$. Now for each vertex $v$ of $\tilde{\tau}_{1} \subseteq \tilde{\sigma} \subseteq \Lambda_{\mathbb{R}, z}$, parallel transport $s_{z}$ to $s_{v} \in \Lambda_{\pi(v)} \otimes \mathbb{G}_{m}=\pi^{*}\left(\mathcal{Q}_{\mathscr{P}} \otimes \mathbb{G}_{m}\right)_{v}$ via the image of a path in $\tilde{\sigma}$ joining $z$ to $v$. It is clear that the collection $\left(s_{v}\right)$ determines an element in $P M\left(\tau_{1}\right)$ as in Construction 2.23. Furthermore, this element is independent of the choice of $\sigma$ : because $s_{z}$ is the germ of $s$, a section of $i_{*} \Lambda \otimes \mathbb{G}_{m}$, it is invariant under monodromy about any loop in $W_{e}$. Thus each $s_{v}$ is independent of $\sigma$.

Definition 5.1. By lifted gluing data, we mean a Čech 1 cocycle $\left(s_{e}\right)$ for the open cover $\mathscr{W}=\left\{W_{\tau} \mid \tau \in \mathscr{P}\right\}$ with values in $i_{*} \Lambda \otimes \mathbb{G}_{m}$. For $e: \tau_{1} \rightarrow \tau_{2}, s_{e} \in \Gamma\left(W_{e}, i_{*} \Lambda \otimes \mathbb{G}_{m}\right)$ induces $s_{e} \in P \check{M}\left(\tau_{1}\right)$ by the above discussion, and hence lifted gluing data $\left(s_{e}\right)$ determine open gluing data.

The main point of simplicity is then

Theorem 5.2. Let $(B, \mathscr{P})$ be positive and simple, and let $s$ be open gluing data over an algebraically closed field $k$ satisfying Condition (LC) of Proposition 4.25. Then

1) $s$ is equivalent (Proposition 4.30) to lifted gluing data, and

2) there is a unique normalized section $f \in \Gamma\left(X_{0}(B, \mathscr{P}, s)\right.$, $\left.\mathcal{L S}_{\text {pre, } X_{0}(B, \mathscr{P}, s)}^{+}\right)$, and this section induces a positive log Calabi-Yau structure on $X_{0}(B, \mathscr{P}, s)$.

Remark 5.3. Before embarking on the proof, it is worthwhile giving a quick explanation as to why lifted gluing data are enough. Consider the situation in Example 2.16. Given $e_{i}: \tau \rightarrow \sigma_{i}$, and lifted gluing data $s_{e_{i}} \in \Gamma\left(W_{e_{i}}, i_{*} \Lambda \otimes \mathbb{G}_{m}\right)$, the gluing map is $\Phi_{e_{2} e_{1}}(s)=s_{e_{1}}^{-1} \circ \Phi_{e_{2} e_{1}} \circ s_{e_{2}}$. Now both $s_{e_{1}}$ and $s_{e_{2}}$ can be viewed as elements of $P M(\tau)$, and as such, we also have $\Phi_{e_{2} e_{1}}(s)=s_{e_{1}}^{-1} \circ s_{e_{2}} \circ \Phi_{e_{2} e_{1}}$. However, to view $s_{e_{2}}$ as acting on $V\left(\tau_{1}\right) \subseteq V\left(\sigma_{1}\right)$ instead of $V\left(\tau_{2}\right) \subseteq V\left(\sigma_{2}\right)$, one parallel transports $s_{e_{2}}$ through the vertex $(0,0)$ to determine the action of $s_{e_{2}}$ on the irreducible component of $V\left(\tau_{1}\right)$ corresponding to $(0,0)$, and one parallel transports through the vertex $(0,1)$ to describe the action on the corresponding component. If $s_{e_{2}}$ is not monodromy invariant in a neighbourhood of the singular point, the induced action on $V\left(\tau_{1}\right)$ does not then arise from 
an element of $\Gamma\left(W_{e_{1}}, i_{*} \Lambda \otimes \mathbb{G}_{m}\right)$. The choice of $s_{e_{1}}$ and $s_{e_{2}}$ then allow all possible automorphisms of $V\left(\tau_{1}\right)$.

More generally, the idea will be to try to split up automorphisms of $V(\tau)$ into a product of ones coming from lifted gluing data.

Proof.

1) By the definition of equivalence in Proposition 4.30, we need to find a system $\left(t_{\tau}\right)_{\tau \in \mathscr{P}}$ with $t_{\tau} \in \check{P} \check{M}(\tau)$ for all $\tau \in \mathscr{P} \backslash \mathscr{P}_{\max }$, and $t_{\tau} \in \Lambda_{z} \otimes \mathbb{G}_{m}$ for $z \in \operatorname{Int}(\tau), \tau \in \mathscr{P}_{\max }$, such that for each $f: \tau_{1} \rightarrow \tau_{2}$, $s_{f}^{\prime}=\left.t_{\tau_{1}}^{-1} s_{f} t_{\tau_{2}}\right|_{\tau_{1}}$ is induced by an element of $\Gamma\left(W_{f}, i_{*} \Lambda \otimes \mathbb{G}_{m}\right)$.

Step 1. Let $\tau \in \mathscr{P}, 0<\operatorname{dim} \tau<\operatorname{dim} B$ be given, and let $\Omega_{j} \subset \mathscr{P}_{1}(\tau)$, $R_{j} \subset \mathscr{P}_{n-1}(\tau), \Delta_{j}, \check{\Delta}_{j}$ be the objects associated to $\tau$ by the definition of simplicity (Definition 1.60). Let $e_{i}: \tau \rightarrow \sigma_{i} \in \mathscr{P}_{\max }, i=1,2$. Then for $f \in \Omega_{j}, n_{\omega}^{e_{1} \circ f, e_{2} \circ f}$ and $\mathrm{D}\left(s_{e_{1}}, f, e_{1}\right) / \mathrm{D}\left(s_{e_{2}}, f, e_{2}\right)$ are independent of the choice of $f \in \Omega_{j}$.

Proof of Step 1. The first statement is Remark 1.61, (1). To see that $\mathrm{D}\left(s_{e_{1}}, f, e_{1}\right) / \mathrm{D}\left(s_{e_{2}}, f, e_{2}\right)$ is independent of $f \in \Omega_{i}$, we note it is enough to show $\mathrm{D}\left(s_{e_{1}}, f, e_{1}\right) / \mathrm{D}\left(s_{e_{2}}, f, e_{2}\right)$ is independent of $f$ in $\Omega_{i}$ whenever we have a diagram

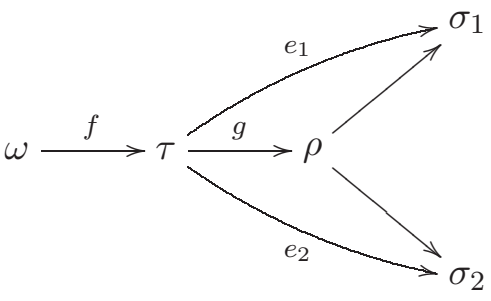

with $\operatorname{dim} \rho=n-1$. If $g \notin R_{i}$, then $n_{g \circ f}=n_{\omega}^{e_{1} \circ f, e_{2} \circ f}=0$ by Def. 1.60, (1), so by Condition (LC), $\mathrm{D}\left(s_{e_{1}}, f, e_{1}\right) / \mathrm{D}\left(s_{e_{2}}, f, e_{2}\right)=1$. Thus it is enough to show this independence for $g \in R_{i}$. Note that by Remark 1.61, (3), we can then assume

$$
\Omega_{i}=\left\{f: \omega \rightarrow \tau \mid n_{g \circ f} \neq 0\right\} .
$$

Now let $z_{i} \in \operatorname{Int}\left(\sigma_{i}\right)$, so we can view $\tilde{\sigma}_{i} \subseteq \Lambda_{\mathbb{R}, z_{i}}$ and $\tilde{\tau}_{i} \subseteq \tilde{\sigma}_{i}$, the face of $\tilde{\sigma}_{i}$ determined by $e_{i}$. As usual, $s_{e_{i}}$ can be viewed as a function $s_{e_{i}}: \check{\Lambda}_{z_{i}} \rightarrow \mathbb{G}_{m}$ which is piecewise multiplicative on the fan $\check{\tau}_{i}^{-1} \check{\Sigma}_{i}$. It is given by $s_{e_{i}}^{v} \in \Lambda_{z_{i}} \otimes \mathbb{G}_{m}$ on the cone of $\check{\tau}_{i}^{-1} \check{\Sigma}_{\sigma_{i}}$ corresponding to a vertex $v$ of $\tilde{\tau}_{i}$. Suppose we are given a cycle of edges $\tilde{\omega}_{1}, \ldots, \tilde{\omega}_{n}$ of $\tilde{\tau}$, with vertices of $\tilde{\omega}_{j}$ being $v_{j}$ and $v_{j+1}$, with $v_{n+1}=v_{1}$. This cycle need not be contained in a 2 -face. Specifying $\tilde{\omega}_{j}$ is the same as specifying an $f_{j}: \omega_{j} \rightarrow \tau$. Then we have (with the signs chosen to correspond to the orientation of the cycle)

$$
\prod_{j=1}^{n} \mathrm{D}\left(s_{e_{i}}, f_{j}, e_{i}\right) \otimes d_{\omega_{j}}^{ \pm 1}=\frac{s_{e_{i}}^{v_{1}}}{s_{e_{i}}^{v_{2}}} \frac{s_{e_{i}}^{v_{2}}}{s_{e_{i}}^{v_{3}}} \cdots \frac{s_{e_{i}}^{v_{n}}}{s_{e_{i}}^{v_{1}}}=1 .
$$


Since $d_{\omega_{j}}$ is monodromy invariant in a neighbourhood of $\operatorname{Int}(\tau)$, we can parallel transport this identity for $i=2$ into $\Lambda_{z_{1}} \otimes \mathbb{G}_{m}$, and so obtain

$$
\prod_{j=1}^{n} \frac{\mathrm{D}\left(s_{e_{1}}, f_{j}, e_{1}\right)}{\mathrm{D}\left(s_{e_{2}}, f_{j}, e_{2}\right)} \otimes d_{\omega_{j}}^{ \pm 1}=1
$$

Note that if $f_{j} \notin \Omega_{i}$, then $n_{g \circ f_{j}}=0$, and by Condition (LC), $\mathrm{D}\left(s_{e_{1}}, f_{j}, e_{1}\right) / \mathrm{D}\left(s_{e_{2}}, f_{j}, e_{2}\right)=1$, so only those $j$ with $f_{j} \in \Omega_{i}$ contribute to the above product.

Now define a piecewise linear function $\varphi$ on the fan $\check{\Sigma}_{\tau}$ by

$$
\varphi(x)=-\inf \left\{\langle y, x\rangle \mid x \in \Delta_{i}\right\}
$$

(See Remark 1.59.) Then $\varphi$ is given by a linear function $q_{h} \in \Lambda_{\tau}$ on each maximal cone of $\check{\Sigma}_{\tau}$ corresponding to $h: v \rightarrow \tau$. As in Remark 1.59, we then have $m_{g \circ h_{1}, g \circ h_{2}}^{\rho}=q_{h_{2}}-q_{h_{1}} \in \Lambda_{\tau}$. Now there is a fan $\check{\Sigma}_{\tau}^{\prime}$, consisting of (not necessarily strictly convex) cones which are the maximal domains of linearity of $\varphi$. The fan $\check{\Sigma}_{\tau}$ is a refinement of $\check{\Sigma}_{\tau}^{\prime}$. There is a one-to-one order reversing correspondence between cones of $\check{\Sigma}_{\tau}^{\prime}$ and faces of $\Delta_{i}$. Of course there is also the usual one-to-one order reversing correspondence between cones of $\check{\Sigma}_{\tau}$ and faces of $\tilde{\tau}$. This gives, for every face $\eta \subseteq \tilde{\tau}$, a face $\eta^{\prime} \subseteq \Delta_{i}$, as follows. The face $\eta$ corresponds to a cone $K_{\eta}$ in $\check{\Sigma}_{\tau}$, which is contained in a cone $K_{\eta}^{\prime}$ of $\check{\Sigma}_{\tau}^{\prime}$, that corresponds to a face $\eta^{\prime} \subseteq \Delta_{i}$. This correspondence does not increase dimensions, i.e., $\Delta_{i}$ is obtained by partial collapse from $\tilde{\tau}$. In particular, an edge of $\tilde{\tau}$ corresponding to $f \in \mathscr{P}_{1}(\tau)$ is mapped to an edge of $\Delta_{i}$ if and only if $f \in \Omega_{i}$. Otherwise it is mapped to a vertex. If $f_{j}: \omega_{j} \rightarrow \tau, j=1,2$, map to the same edge of $\Delta_{i}$, then we can find a cycle in $\tilde{\tau}$ of edges containing $f_{1}$ and $f_{2}$ and such that all other edges of the cycle map to vertices of $\Delta_{i}$. Then $d_{\omega_{1}}= \pm d_{\omega_{2}}=: d$ is parallel to the corresponding edge of $\Delta_{i}$, and $\omega_{1}$ and $\omega_{2}$ are traversed in the cycle in opposite directions, so that (5.1) yields

$$
\left(\frac{\mathrm{D}\left(s_{e_{1}}, f_{1}, e_{1}\right)}{\mathrm{D}\left(s_{e_{2}}, f_{1}, e_{2}\right)} \otimes d\right) \cdot\left(\frac{\mathrm{D}\left(s_{e_{1}}, f_{2}, e_{1}\right)}{\mathrm{D}\left(s_{e_{2}}, f_{2}, e_{2}\right)} \otimes d\right)^{-1}=1
$$

Thus $\mathrm{D}\left(s_{e_{1}}, f_{j}, e_{1}\right) / \mathrm{D}\left(s_{e_{2}}, f_{j}, e_{2}\right)$ is independent of $j$.

If $\operatorname{dim} \Delta_{i}=1$, then we are now done. Otherwise, any two-face $\eta$ of $\Delta_{i}$ is an elementary, hence standard, simplex. Thus we can find a cycle of edges of $\tilde{\tau}$ with only three of its edges $f_{j}: \omega_{j} \rightarrow \tau(j=1,2,3)$ in $\Omega_{i}$, and such that these edges map to the three edges of $\eta$. Also, $d_{\omega_{1}}, d_{\omega_{2}}$ and $d_{\omega_{3}}$ span the tangent space to $\eta$, so without loss of generality we can take (in a suitable basis) $d_{\omega_{1}}=(1,0), d_{\omega_{2}}=(0,1)$ and $d_{\omega_{3}}=(-1,-1)$. 
Then (5.1) is equivalent to

$$
\begin{aligned}
\left(\frac{\mathrm{D}\left(s_{e_{1}}, f_{1}, e_{1}\right)}{\mathrm{D}\left(s_{e_{2}}, f_{1}, e_{2}\right)} \otimes(1,0)\right) & \left(\frac{\mathrm{D}\left(s_{e_{1}}, f_{2}, e_{1}\right)}{\mathrm{D}\left(s_{e_{2}}, f_{2}, e_{2}\right)} \otimes(0,1)\right) \\
\cdot\left(\frac{\mathrm{D}\left(s_{e_{1}}, f_{3}, e_{1}\right)}{\mathrm{D}\left(s_{e_{2}}, f_{3}, e_{2}\right)} \otimes(-1,-1)\right) & =1,
\end{aligned}
$$

which implies $\mathrm{D}\left(s_{e_{1}}, f_{j}, e_{1}\right) / \mathrm{D}\left(s_{e_{2}}, f_{j}, e_{2}\right)$ is independent of $j$.

Since this is true of every 2 -face of $\Delta_{i}$, one sees $\mathrm{D}\left(s_{e_{1}}, f, e_{1}\right) /$ $\mathrm{D}\left(s_{e_{2}}, f, e_{2}\right)$ is independent of $f \in \Omega_{i}$, as desired. This proves the claim.

Step 2. Let $\tau \in \mathscr{P}$ with $0<\operatorname{dim} \tau<\operatorname{dim} B$, and fix some $e: \tau \rightarrow$ $\sigma \in \mathscr{P}_{\max }, z \in \operatorname{Int}(\sigma)$. Then there is an element $u_{\tau} \in \Lambda_{z} \otimes \mathbb{G}_{m}(k)$, or alternatively a homomorphism $u_{\tau}: \check{\Lambda}_{z} \rightarrow \mathbb{G}_{m}(k)$, with the property that for every $e^{\prime}: \tau \rightarrow \sigma^{\prime} \in \mathscr{P}_{\max }$ and $f: \omega \rightarrow \tau$ with $\operatorname{dim} \omega=1$,

$$
u_{\tau}\left(n_{\omega}^{e \circ f, e^{\prime} \circ f}\right)=\frac{\mathrm{D}\left(s_{e^{\prime}}, f, e^{\prime}\right)}{\mathrm{D}\left(s_{e}, f, e\right)} s_{e}\left(n_{\omega}^{e \circ f, e^{\prime} \circ f}\right)^{-1} .
$$

Proof of Step 2. Let $\Omega_{i} \subseteq \mathscr{P}_{1}(\tau), R_{i} \subseteq \mathscr{P}_{n-1}(\tau), \Delta_{i}, \check{\Delta}_{i}$ be as in Definition 1.60. First, whenever $n_{\omega}^{e \circ f, e^{\prime} \circ f}=0$, Condition (LC) implies

$$
\mathrm{D}\left(s_{e^{\prime}}, f, e^{\prime}\right) / \mathrm{D}\left(s_{e}, f, e\right)=1,
$$

so (5.2) holds for any choice of $u_{\tau}$. Furthermore, if $f: \omega \rightarrow \tau$ is not in $\bigcup_{i=1}^{p} \Omega_{i}$, then by Remark 1.61, (2) $n_{\omega}^{e \circ f, e^{\prime} \circ f}=0$. Thus we don't need to worry about such $f$.

Next, let $T_{\check{\Delta}_{i}} \subseteq \mathcal{Q}_{\tau, \mathbb{R}}^{*} \subseteq \check{\Lambda}_{\mathbb{R}, z}$ denote the tangent space to the polytope $\check{\Delta}_{i}$. By Remark 1.61, (4), $T_{\check{\Delta}_{1}}+\cdots+T_{\check{\Delta}_{p}}$ form an interior direct sum in $\check{\Lambda}_{\mathbb{R}, z}$. Thus for any collection of homomorphisms $u_{i}: T_{\check{\Delta}_{i}} \cap \check{\Lambda}_{z} \rightarrow \mathbb{G}_{m}(k)$, $i=1, \ldots, p$, there is a homomorphism $u: \check{\Lambda}_{z} \rightarrow \mathbb{G}_{m}(k)$ extending each of the $u_{i}$ 's. (Here we use the fact that $\mathbb{G}_{m}(k)$ is divisible, so that any homomorphism defined on a sublattice of $\check{\Lambda}_{z}$ extends to one on $\check{\Lambda}_{z}$. It is here we use that $k$ is algebraically closed.) Thus, since for $f: \omega \rightarrow \tau$ in $\Omega_{i}, n_{\omega}^{e \circ f, e^{\prime} \circ f} \in T_{\check{\Delta}_{i}} \cap \check{\Lambda}_{z}$, it will be enough to show that we can construct $u_{i}: T_{\check{\Delta}_{i}} \cap \check{\Lambda}_{z} \rightarrow \mathbb{G}_{m}$ such that

$$
u_{i}\left(n_{\omega}^{e \circ f, e^{\prime} \circ f}\right)=\frac{\mathrm{D}\left(s_{e^{\prime}}, f, e^{\prime}\right)}{\mathrm{D}\left(s_{e}, f, e\right)} s_{e}\left(n_{\omega}^{e \circ f, e^{\prime} \circ f}\right)^{-1}
$$

for all $f: \omega \rightarrow \tau$ in $\Omega_{i}$, for all $e^{\prime}: \tau \rightarrow \sigma^{\prime}$.

By Step 1, $n_{\omega}^{e \circ f, e^{\prime} \circ f}$ and $\mathrm{D}\left(s_{e^{\prime}}, f, e^{\prime}\right) / \mathrm{D}\left(s_{e}, f, e\right)$ are independent of $f \in \Omega_{i}$. Because $\check{\Delta}_{i}$ is an elementary simplex which is the convex hull of $\left\{n_{\omega}^{e o f, e^{\prime} \circ f} \mid e^{\prime}: \tau \rightarrow \sigma^{\prime}\right\}$, the non-zero elements of this set are linearly independent. In addition, if $n_{\omega}^{e \circ f, e^{\prime} \circ f}=n_{\omega}^{e \circ f, e^{\prime \prime} \circ f}$, then $0=$ 
$n_{\omega}^{e \circ f, e^{\prime \prime} \circ f}-n_{\omega}^{e \circ f, e^{\prime} \circ f}=n_{\omega}^{e^{\prime} \circ f, e^{\prime \prime} \circ f}$, and Condition (LC) then shows that

$$
\frac{\mathrm{D}\left(s_{e^{\prime}}, f, e^{\prime}\right)}{\mathrm{D}\left(s_{e}, f, e\right)} s_{e}\left(n_{\omega}^{e \circ f, e^{\prime} \circ f}\right)^{-1}=\frac{\mathrm{D}\left(s_{e^{\prime \prime}}, f, e^{\prime \prime}\right)}{\mathrm{D}\left(s_{e}, f, e\right)} s_{e}\left(n_{\omega}^{e \circ f, e^{\prime \prime} \circ f}\right)^{-1} .
$$

Thus there exists a homomorphism $u_{i}: T_{\check{\Delta}_{i}} \cap \check{\Lambda}_{z} \rightarrow \mathbb{G}_{m}$ satisfying (5.3) and hence a homomorphism $u_{\tau}: \check{\Lambda}_{z} \rightarrow \mathbb{G}_{m}$ satisfying (5.2).

Step 3. The open gluing data $\left(s_{e}\right)$ are equivalent to lifted gluing data.

Proof of Step 3. We construct $\left(t_{\tau}\right)_{\tau \in \mathscr{P}}$ as follows. For $\tau \in \mathscr{P}_{\max }$ or $\operatorname{dim} \tau=0$, set $t_{\tau}=1$. For any other $\tau$, fix $e: \tau \rightarrow \sigma \in \mathscr{P}_{\max }$ and take $t_{\tau}=s_{e} u_{\tau}$ with $u_{\tau}$ as in Step 2. We need to show for each $f: \tau_{1} \rightarrow \tau_{2}$, $s_{f}^{\prime}=\left.t_{\tau_{1}}^{-1} s_{f} t_{\tau_{2}}\right|_{\tau_{1}}$ is induced from $\Gamma\left(W_{f}, i_{*} \Lambda \otimes \mathbb{G}_{m}(k)\right)$.

First note that for $f: \tau_{1} \rightarrow \tau_{2}, s \in P M\left(\tau_{1}\right)$ is induced by an element of $\Gamma\left(W_{f}, i_{*} \Lambda \otimes \mathbb{G}_{m}(k)\right)$ if and only if for all $g: \omega \rightarrow \tau_{1}$ with $\operatorname{dim} \omega=1$ and $e: \tau_{2} \rightarrow \sigma \in \mathscr{P}_{\max }, \mathrm{D}(s, g, e \circ f)=1$. Indeed, this says that for any vertex $v$ of $\tilde{\tau}_{1}$ and any $\sigma \in \mathscr{P}_{\max }$ containing $\tau_{2}$, the parallel transport of $s^{v} \in \Lambda_{\pi(v)} \otimes \mathbb{G}_{m}(k)$ into $z \in \operatorname{Int}(\sigma)$ is independent of $v$. This gives a well-defined element of $\Lambda_{z} \otimes \mathbb{G}_{m}(k)$, which is then invariant under monodromy in $W_{f}$, as desired.

We first consider $e^{\prime}: \tau \rightarrow \sigma^{\prime} \in \mathscr{P}_{\max }$, and wish to show $s_{e^{\prime}}^{\prime}=t_{\tau}^{-1} s_{e^{\prime}}$ is in $\Gamma\left(W_{e^{\prime}}, i_{*} \Lambda \otimes \mathbb{G}_{m}(k)\right)$. This is trivial if $\operatorname{dim} \tau=0$. Otherwise, applying Remark 3.26 to both $s_{e}$ and $u_{\tau}$ for $g: \omega \rightarrow \tau$, $\operatorname{dim} \omega=1$, and the definition of $u_{\tau}(5.2)$, and $\mathrm{D}\left(u_{\tau}, g, e\right)=1$

$$
\begin{aligned}
\mathrm{D} & \left(s_{e^{\prime}}^{\prime}, g, e^{\prime}\right)=\mathrm{D}\left(t_{\tau}, g, e^{\prime}\right)^{-1} \mathrm{D}\left(s_{e^{\prime}}, g, e^{\prime}\right) \\
& =\mathrm{D}\left(s_{e}, g, e^{\prime}\right)^{-1} \mathrm{D}\left(u_{\tau}, g, e^{\prime}\right)^{-1} \mathrm{D}\left(s_{e^{\prime}}, g, e^{\prime}\right) \\
& =\mathrm{D}\left(s_{e}, g, e\right)^{-1} s_{e}\left(n_{\omega}^{e \circ g, e^{\prime} \circ g}\right)^{-1} \mathrm{D}\left(u_{\tau}, g, e\right)^{-1} u_{\tau}\left(n_{\omega}^{e \circ g, e^{\prime} \circ g}\right)^{-1} \mathrm{D}\left(s_{e^{\prime}}, g, e^{\prime}\right) \\
& =\mathrm{D}\left(s_{e}, g, e\right)^{-1} s_{e}\left(n_{\omega}^{e \circ g, e^{\prime} \circ g}\right)^{-1} \frac{\mathrm{D}\left(s_{e}, g, e\right)}{\mathrm{D}\left(s_{e^{\prime}}, g, e^{\prime}\right)} s_{e}\left(n_{\omega}^{e \circ g, e^{\prime} \circ g}\right) \mathrm{D}\left(s_{e^{\prime}}, g, e^{\prime}\right) \\
& =1
\end{aligned}
$$

as desired. Thus $s_{e^{\prime}}^{\prime} \in \Gamma\left(W_{e^{\prime}}, i_{*} \Lambda \otimes \mathbb{G}_{m}\right)$ for any $e^{\prime}: \tau \rightarrow \sigma^{\prime} \in \mathscr{P}_{\max }$.

On the other hand, for $\tau_{1} \stackrel{f}{\longrightarrow} \tau_{2} \stackrel{e^{\prime}}{\longrightarrow} \sigma^{\prime}, s_{e^{\prime} \circ f}^{\prime}\left(\left.s_{e^{\prime}}^{\prime}\right|_{\tau_{1}}\right)^{-1}=s_{f}^{\prime}$. But then for any $g: \omega \rightarrow \tau_{1}$,

$$
\begin{aligned}
\mathrm{D}\left(s_{f}^{\prime}, g, e^{\prime} \circ f\right) & =\mathrm{D}\left(s_{e^{\prime} \circ f}^{\prime}, g, e^{\prime} \circ f\right) \mathrm{D}\left(\left.s_{e^{\prime}}^{\prime}\right|_{\tau_{1}} ^{-1}, g, e^{\prime} \circ f\right) \\
& =\mathrm{D}\left(s_{e^{\prime} \circ f}^{\prime}, g, e^{\prime} \circ f\right) \mathrm{D}\left(s_{e^{\prime}}^{\prime}, f \circ g, e^{\prime}\right)^{-1} \\
& =1
\end{aligned}
$$

by the previous case. Thus $s_{f}^{\prime} \in \Gamma\left(W_{f}, i_{*} \Lambda \otimes \mathbb{G}_{m}\right)$. So we conclude that $s^{\prime}$ is lifted gluing data.

2) Given $s, X=X_{0}(B, \mathscr{P}, s)$, it is clear there is a unique normalized section $f \in \Gamma\left(X, \mathcal{L S}_{\mathrm{pre}, X}^{+}\right)$. Indeed, using the notation of the proof of 
Proposition 4.25, with $e: \omega \rightarrow \sigma$,

$$
f_{\sigma, e}=\sum_{p \in \check{\Delta}(\omega) \cap \check{\Lambda}_{y}} f_{\sigma, e, p} z^{p},
$$

$f_{\sigma, e, p}$ is determined by the normalization condition whenever $p$ is a vertex of $\breve{\Delta}(\omega)$. But since $\breve{\Delta}(\omega)$ is an elementary simplex, all integral points of $\breve{\Delta}(\omega)$ are vertices, so $f_{\sigma, e}$ is completely determined, and $f$ is unique. It remains to show $f$ defines a log Calabi-Yau structure by checking the multiplicative condition of Theorem 3.22.

Focus on one $\sigma \in \mathscr{P}_{\max }$ and $e: \tau \rightarrow \sigma$ with $\operatorname{dim} \tau=2$. For $g: \omega \rightarrow \tau$ with $\operatorname{dim} \omega=1$, note that $\mathrm{D}\left(s_{e^{\prime}}, g, e^{\prime}\right)=1$ for all $e^{\prime}: \tau \rightarrow \sigma^{\prime} \in \mathscr{P}_{\max }$ because the gluing data are lifted. It thus follows from (4.4) in the proof of Proposition 4.25 that $f_{\sigma, e \circ g, n_{\omega}^{e \circ g, e^{\prime} \circ g}}=s_{e}\left(n_{\omega}^{e \circ g, e^{\prime} \circ g}\right)$, and in particular

$$
\left.f_{\sigma, e \circ g}\right|_{V_{e}}=\sum_{p \in \check{\Delta}_{i} \cap \check{\Lambda}_{y}} s_{e}(p) z^{p}
$$

if $g \in \Omega_{i}$, where $\check{\Delta}_{i}, \Omega_{i}$ are associated with $\tau$. Thus $\left.f_{\sigma, e \circ g}\right|_{V_{e}}$ only depends on which $\Omega_{i}$ has $g$ as an element.

Now as $\tau$ is two-dimensional, we have $p \leq 2$ in the definition of simplicity for $\tau$ by Remark 1.61, (5). If $p=0$, then $n_{\omega}^{e \circ g, e^{\prime} \circ g}=0$ and $f_{\sigma, e \circ g}=1$ for all $g: \omega \rightarrow \tau$. Thus the multiplicative condition is trivial. If $p=1$, then $\operatorname{dim} \Delta_{1}=1$ or 2 , and is either a line segment of length 1 or a two-dimensional elementary simplex. Then

$$
\Omega_{1}=\left\{g_{i}: \omega_{i} \rightarrow \tau \mid 1 \leq i \leq r\right\}
$$

with $r=2$ or 3 in the two cases (if $r=2, g_{1}$ and $g_{2}$ should be thought of as opposite parallel edges of $\tau$ ). Note $\left.f_{\sigma, e \circ g}\right|_{V_{e}}=1$ for all $g \notin \bigcup_{i=1}^{p} \Omega_{i}$. If $\operatorname{dim} \Delta_{1}=1$, then $d_{\omega_{1}}= \pm d_{\omega_{2}}$, and the multiplicative condition becomes

$$
\left(\left.f_{\sigma, e \circ g_{1}}\right|_{V_{e}} \otimes d_{\omega_{1}}\right) \cdot\left(\left.f_{\sigma, e \circ g_{2}}\right|_{V_{e}} \otimes d_{\omega_{1}}\right)^{-1}=1,
$$

which holds automatically as $\left.f_{\sigma, e o g}\right|_{V_{e}}$ is independent of $g \in \Omega_{i}$, as remarked above. If $\operatorname{dim} \Delta_{1}=2$, we can without loss of generality assume $d_{\omega_{1}}=(1,0), d_{\omega_{2}}=(0,1)$ and $d_{\omega_{3}}=(-1,-1)$ (inside the twodimensional space $\left.\Lambda_{\tau}\right)$. The multiplicative condition then becomes

$$
\left(\left.f_{\sigma, e \circ g_{1}}\right|_{V_{e}}, 1\right) \cdot\left(1,\left.f_{\sigma, e \circ g_{2}}\right|_{V_{e}}\right) \cdot\left(\left.f_{\sigma, e \circ g_{3}}\right|_{V_{e}},\left.f_{\sigma, e \circ g_{3}}\right|_{V_{e}}\right)^{-1}=1
$$

which again holds.

If $p=2$, then $\Delta_{1}$ and $\Delta_{2}$ are both line segments, which without loss of generality we can take to be the line segments with endpoints $(0,0)$ and $(1,0)$ for $\Delta_{1}$ and $(0,0)$ and $(a, b)$ for $\Delta_{2}$ with $a, b \in \mathbb{Z}, \operatorname{gcd}(a, b)=1$. Again $\Omega_{j}=\left\{g_{i}^{j}: \omega_{i}^{j} \rightarrow \tau \mid i=1,2\right\}, j=1,2$ (with $\omega_{1}^{j}, \omega_{2}^{j}$ parallel and opposite edges of $\tau$ ). Then writing $f_{j}=f_{\sigma, e \circ g_{i}^{j}}$ for $j=1,2$, independent of $i$, the multiplicative condition becomes, without loss of generality,

$$
\left(f_{1}, 1\right) \cdot\left(f_{2}^{a}, f_{2}^{b}\right) \cdot\left(f_{1}^{-1}, 1\right) \cdot\left(f_{2}^{-a}, f_{2}^{-b}\right)=1,
$$


which again holds. This completes the proof of Theorem 5.2. $\quad$ q.e.d.

Theorem 5.4. Given $(B, \mathscr{P})$ positive and simple, the set of positive $\log$ Calabi-Yau spaces with dual intersection complex $(B, \mathscr{P})$, modulo isomorphism preserving $B$, is $H^{1}\left(\mathscr{W}, i_{*} \Lambda \otimes \mathbb{G}_{m}\right)$.

Proof. If $X$ is a positive log Calabi-Yau space, there exist open gluing data $s$ such that $X=X_{0}(B, \mathscr{P}, s)$ by Theorem 4.14, and $s$ then satisfies Condition (LC) by Proposition 4.25. Thus by Theorem 5.2, (1), $s$ is equivalent to lifted gluing data.

On the other hand, any lifted gluing data trivially satisfy Condition (LC), hence define a positive log Calabi-Yau space by Theorem $5.2,(2)$. Now if $s, s^{\prime}$ are two choices of lifted gluing data defining log Calabi-Yau spaces $X$ and $X^{\prime}$ which are isomorphic via an isomorphism preserving $B$, then by Proposition 4.30, $s$ and $s^{\prime}$ are equivalent, i.e., there exists $\left(t_{\tau}\right)$ such that $s_{e}^{\prime}=t_{\tau_{1}}^{-1} s_{e} t_{\tau_{2}}$ for $e: \tau_{1} \rightarrow \tau_{2}$. In particular, for $e: \tau \rightarrow \sigma \in \mathscr{P}_{\max }, t_{\tau}=\left(s_{e} / s_{e}^{\prime}\right) t_{\sigma}$. But $s_{e}, s_{e}^{\prime} \in \Gamma\left(W_{e}, i_{*} \Lambda \otimes \mathbb{G}_{m}\right)$, and $t_{\sigma} \in \Gamma\left(\operatorname{Int}(\sigma), i_{*} \Lambda \otimes \mathbb{G}_{m}\right)$. Thus $t_{\tau} \in \Gamma\left(W_{e} \cap \operatorname{Int}(\sigma), i_{*} \Lambda \otimes \mathbb{G}_{m}\right)$. Since $t_{\tau}$ is independent of $\sigma$, in fact $t_{\tau} \in \Gamma\left(W_{\tau}, i_{*} \Lambda \otimes \mathbb{G}_{m}\right)$. Thus $s$ and $s^{\prime}$ are cohomologous as Cech 1-cocycles with respect to the open covering $\mathscr{W}$.

Conversely, if $s$ and $s^{\prime}$ are lifted gluing data which are cohomologous, then by Theorem $2.32, X_{0}(B, \mathscr{P}, s)$ and $X_{0}\left(B, \mathscr{P}, s^{\prime}\right)$ are isomorphic. This isomorphism is induced on open sets $V(\sigma)$ by $t_{\sigma}$, where $\left(t_{\tau}\right)_{\tau \in \mathscr{P}}$ is the Cech 0-cochain making $s$ and $s^{\prime}$ cohomologous. But the pull-back of a normalized log structure under an element of $\Gamma\left(W_{\sigma}, i_{*} \Lambda \otimes \mathbb{G}_{m}(S)\right)$ is still normalized, so the pull-back of the normalized log structure on $X_{0}\left(B, \mathscr{P}, s^{\prime}\right)$ is a normalized log structure on $X_{0}(B, \mathscr{P}, s)$. Hence by the uniqueness result of Theorem 5.2, $(2), X_{0}(B, \mathscr{P}, s)$ and $X_{0}\left(B, \mathscr{P}, s^{\prime}\right)$ are isomorphic as log Calabi-Yau spaces.

q.e.d.

We finally note that this Čech cohomology group computes the cohomology of $i_{*} \Lambda \otimes \mathbb{G}_{m}$ :

Lemma 5.5. Suppose the discriminant locus of $B$ is straightened (Remark 1.49). Then $\mathscr{W}$ is an acyclic cover for both $i_{*} \Lambda$ and $i_{*} \Lambda \otimes$ $\mathbb{G}_{m}(S)$. In particular,

$$
H^{j}\left(\mathscr{W}, i_{*} \Lambda\right)=H^{j}\left(B, i_{*} \Lambda\right)
$$

and

$$
H^{j}\left(\mathscr{W}, i_{*} \Lambda \otimes \mathbb{G}_{m}(S)\right)=H^{j}\left(B, i_{*} \Lambda \otimes \mathbb{G}_{m}(S)\right)
$$

for all $j \geq 0$.

Proof. We show $H^{j}\left(W_{\tau_{1} \cdots \tau_{p}}, i_{*} \Lambda\right)=0$ and $H^{j}\left(W_{\tau_{1} \cdots \tau_{p}}, i_{*} \Lambda \otimes \mathbb{G}_{m}(S)\right)$ $=1$ for $j>0$ only for $p=1$ for convenience. The more general case is the same, keeping in mind that $W_{\tau_{1} \cdots \tau_{p}}$ may have a number of connected components. 
The first thing to observe is that $W_{\tau}$ and $\Delta \cap W_{\tau}$ are both topological cones over the point $x=\operatorname{Bar}(\tau)$. In particular, there is a fundamental system of neighbourhoods $\left\{W_{j}\right\}$ of the barycenter $x$ of $\tau$ and homeomorphisms $\varphi_{j}: W_{j} \rightarrow W_{\tau}$ (just dilations) such that $\varphi_{j}^{-1}\left(W_{\tau} \cap \Delta\right)=W_{j} \cap \Delta$ and $\left.\varphi_{j}^{*} \Lambda\right|_{W_{\tau} \backslash \Delta}=\left.\Lambda\right|_{W_{j} \backslash \Delta}$. Thus the restriction maps

$$
H^{p}\left(W_{\tau}, i_{*} \Lambda\right) \longrightarrow H^{p}\left(W_{j}, i_{*} \Lambda\right)
$$

are isomorphisms for all $j$. Thus $H^{p}\left(W_{\tau}, i_{*} \Lambda\right)$ is isomorphic to the stalk of $R^{p} \operatorname{id}_{*}\left(i_{*} \Lambda\right)$ at $x$. Of course, $R^{p} \mathrm{id}_{*}$ of any abelian sheaf vanishes for $p>0$ because it is computed by applying $\mathrm{id}_{*}$ to an injective resolution. In particular $H^{p}\left(W_{\tau}, i_{*} \Lambda\right)=0$ for all $p>0$. Similarly $H^{p}\left(W_{\tau}, i_{*} \Lambda \otimes\right.$ $\left.\mathbb{G}_{m}(S)\right)=1$ for all $p>0$.

q.e.d.

Remark 5.6. If $\Delta=\emptyset$, then $(B, \mathscr{P})$ is automatically simple, and the above discussion can be interpreted as follows. There is in fact an exact sequence

$$
0 \longrightarrow \Lambda_{\mathscr{P}} \longrightarrow \Lambda \longrightarrow \mathcal{Q}_{\mathscr{P}} \longrightarrow 0,
$$

and hence an exact sequence

$$
H^{1}\left(B, \Lambda_{\mathscr{P}} \otimes \mathbb{G}_{m}(S)\right) \stackrel{f_{1}}{\longrightarrow} H^{1}\left(B, \Lambda \otimes \mathbb{G}_{m}(S)\right) \stackrel{f_{2}}{\longrightarrow} H^{1}\left(B, \mathcal{Q}_{\mathscr{P}} \otimes \mathbb{G}_{m}(S)\right) .
$$

The map $f_{2}$ can be interpreted as taking lifted gluing data to the corresponding closed gluing data; elements of $H^{1}\left(B, \mathcal{Q} \mathscr{P} \otimes \mathbb{G}_{m}(S)\right)$ represent closed gluing. The kernel of $f_{2}$ can then be viewed as the space of log smooth structures on the space $X_{0}(B, \mathscr{P}, 1)$, where 1 denotes the trivial gluing data. This coincides with $\operatorname{im} f_{1}$. This interpretation is not so simple for the case when $\Delta \neq \emptyset$, since then (5.4) fails to be exact on $\Delta$. However, there is always a map

$$
H^{1}\left(B, i_{*} \Lambda \otimes \mathbb{G}_{m}(S)\right) \longrightarrow H^{1}\left(B, \mathcal{Q}_{\mathscr{P}} \otimes \mathbb{G}_{m}(S)\right)
$$

taking lifted gluing data to closed gluing data, and in the simple case the kernel again represents log Calabi-Yau structures on the trivially glued $X_{0}(B, \mathscr{P}, 1)$.

Example 5.7. Following up on Example 1.5, (3), consider $\Gamma \subseteq G$ defined by, for $e$ a positive integer,

$$
\Gamma=\left\{\begin{array}{l|l}
A_{u, v} \in \operatorname{Aff}\left(M_{\mathbb{R}}\right) & \begin{array}{l}
\text { for }(u, v) \text { in the lattice generated by } \\
(e, 0) \text { and }(-e(e-1) / 2, e)
\end{array}
\end{array}\right\} .
$$

The generators of $\Gamma$ are $T_{1}$ and $T_{2}$ with

$$
\begin{aligned}
& T_{1}\left(m_{1}, m_{2}\right)=\left(m_{1}+e, m_{2}\right) \\
& T_{2}\left(m_{1}, m_{2}\right)=\left(m_{1}+e m_{2}, m_{2}+e\right) .
\end{aligned}
$$

These are integral not only with respect to the lattice $M \subseteq M_{\mathbb{R}}$, but also the lattice $e M \subseteq M_{\mathbb{R}}$. Changing basis of $M_{\mathbb{R}}$ from the standard 
basis $e_{1}, e_{2}$ to $e e_{1}, e e_{2}$, we can just as well work with the generators

$$
\begin{aligned}
& T_{1}\left(m_{1}, m_{2}\right)=\left(m_{1}+1, m_{2}\right) \\
& T_{2}\left(m_{1}, m_{2}\right)=\left(m_{1}+e m_{2}, m_{2}+1\right),
\end{aligned}
$$

integral with respect to the lattice $M \subseteq M_{\mathbb{R}}$.

Let $B=M_{\mathbb{R}} / \Gamma$. Take as a fundamental domain for the action of $\Gamma$ the unit square with vertices $(0,0),(1,0),(0,1)$ and $(1,1)$, and obtain a polyhedral decomposition $\mathscr{P}$ of $B$ by triangulating this square by adding an edge joining $(0,0)$ and $(1,1)$. Then $\mathscr{P}$ contains one vertex $v$, and $\Sigma_{v}$ is the fan with rays generated by $(1,0),(1,1),(0,1),(-1,0)$, $(e-1,-1)$ and $(e,-1)$. Then $X_{v}$ can be viewed as a Hirzebruch surface $F_{e}$ blown up at two points, while $X_{0}(B, \mathscr{P}, s)$ is obtained by identifying "opposite" toric divisors of this surface. The moduli space of log CalabiYau spaces with dual intersection complex $(B, \mathscr{P})$ is then $H^{1}(B, \Lambda \otimes$ $\left.\mathbb{G}_{m}(k)\right)$. Now $\Lambda$ is a local system with monodromy $\left(\begin{array}{ll}1 & 0 \\ 0 & 1\end{array}\right)$ and $\left(\begin{array}{ll}1 & e \\ 0 & 1\end{array}\right)$ about the two generators corresponding to $T_{1}$ and $T_{2}$, and in view of Lemma 5.5 it is straightforward to calculate that $H^{1}\left(B, \Lambda \otimes \mathbb{G}_{m}(k)\right)=$ $\left(\mathbb{G}_{m}(k)\right)^{2} \times \mu_{e}$, where $\mu_{e}$ denotes the group of eth roots of unity in $k$. This recovers a calculation of $[\mathbf{4 3}]$, and demonstrates that there may be several connected components to the moduli space.

For future use, we note

Corollary 5.8. Let $(B, \mathscr{P})$ be positive and simple, and s lifted gluing data, inducing a log Calabi-Yau structure on $X_{0}(B, \mathscr{P}, s)$. Then the log-singular set $Z \subseteq X_{0}(B, \mathscr{P}, s)$ can be taken so that for any $\tau \in \mathscr{P}$, $q_{\tau}: X_{\tau} \rightarrow X_{0}(B, \mathscr{P}, s), q_{\tau}^{-1}(Z)=Z_{1} \cup \cdots \cup Z_{p} \cup Z^{\prime}$, where the codimension of $Z^{\prime}$ in $X_{\tau}$ is at least two, $Z^{\prime}$ is contained in the complement of the big torus orbit of $X_{\tau}$, and $Z_{1}, \ldots, Z_{p}$ are irreducible and reduced Cartier divisors on $X_{\tau}$ which are linearly independent in $\operatorname{Pic}\left(X_{\tau}\right)$. Furthermore the Newton polytope of $Z_{i}$ is $\breve{\Delta}_{i}$, where $\breve{\Delta}_{1}, \ldots, \breve{\Delta}_{p}$ are as in Definition 1.60 .

Proof. $q_{\tau}^{-1}(Z)$ consists of various irreducible components arising as follows. The open gluing data $s$ induce a section $f \in \Gamma\left(X_{0}(B, \mathscr{P}, s)\right.$, $\left.\mathcal{L S}_{\text {pre }, X_{0}(B, \mathscr{P}, s)}^{+}\right)$which induces sections of the line bundle $\mathcal{N}_{\omega}$ on $X_{\omega}$ for each $\omega \in \mathscr{P}$ with $\operatorname{dim} \omega=1$. Set $Z_{\omega}$ to be the zero locus of this section. Then

$$
Z=\bigcup_{\substack{\omega \in \mathscr{P} \\ \operatorname{dim} \omega=1}} q_{\omega}\left(Z_{\omega}\right)
$$

Thus $q_{\tau}^{-1}(Z)$ contains the codimension one components

$$
\left\{F_{S, \bar{s}}(e)^{-1}\left(Z_{\omega}\right) \mid e: \omega \rightarrow \tau \text { an element of } \mathscr{P}_{1}(\tau)\right\},
$$

and a number of higher codimension components, contained in the toric boundary of $X_{\tau}$, i.e., the complement of the big torus orbit. 
Now by definition of simplicity, $F_{S, \bar{s}}(e)^{-1}\left(Z_{\omega}\right)=\emptyset$ unless $e \in \bigcup_{i=1}^{p} \Omega_{i}$. Furthermore, if $e \in \Omega_{i}$, then the line bundle $F_{S, \bar{s}}^{*}\left(\mathcal{N}_{\omega}\right)$ has Newton polytope given by the elementary simplex $\check{\Delta}_{i}$. Finally, as in the beginning of the proof of Theorem 5.2, (2), $F_{S, \bar{s}}(e)^{-1}\left(Z_{\omega}\right)$ depends only on the value of $i$ for which we have $e \in \Omega_{i}$. Thus we can write $q_{\tau}^{-1}(Z)=Z_{1} \cup \cdots \cup Z_{p} \cup Z^{\prime}$ where $\operatorname{codim}\left(Z^{\prime}\right) \geq 2$. Also, each $Z_{i}$ is irreducible as it contains no toric stratum of $X_{\tau}$ and comes from an elementary Newton polytope. Finally from Remark 1.61, $T_{\check{\Delta}_{1}}+\cdots+T_{\check{\Delta}_{p}}$ (where $T_{\check{\Delta}_{i}}$ is the tangent space to $\check{\Delta}_{i}$ ) form an interior direct sum in $\mathcal{Q}_{\tau, \mathbb{R}}^{*}$, from which it follows that $Z_{1}, \ldots, Z_{p}$ are linearly independent in $\left(\operatorname{Pic} X_{\tau}\right) \otimes \mathbb{R}$.

q.e.d.

5.2. The logarithmic Picard group in the simple case. L. Illusie [25] has suggested that if $X$ is a log scheme, then $H^{1}\left(X, \mathcal{M}_{X}^{\mathrm{gp}}\right)$ is a good candidate for a logarithmic Picard group. Let us give some motivation for this here. Let $X$ be a normal scheme or algebraic space, $Y \subseteq X$ a Cartier divisor. Now suppose we have an element of $\operatorname{Pic}(X \backslash Y)$ which we wish to extend to $X$. This could be done by taking a specific Weil divisor $D$ on $X \backslash Y$ which is Cartier, representing some element of $\operatorname{Pic}(X \backslash Y)$, and taking its closure $\bar{D}$ in $X$. However, there are two problems. First, the linear equivalence class of $\bar{D}$ might depend on which member of the linear equivalence class of $D$ we chose. It is well-defined only up to Weil divisors supported on $Y$. Second, $\bar{D}$ might be Weil, but not Cartier. Thus we do not get a map $\operatorname{Pic}(X \backslash Y) \rightarrow \operatorname{Pic}(X)$.

On the other hand, if we think in terms of log geometry, and take $j: X \backslash Y \hookrightarrow X$ the inclusion, we set $\mathcal{M}_{(X, Y)}=j_{*} \mathcal{O}_{X \backslash Y}^{\times} \cap \mathcal{O}_{X}$. Then $\mathcal{M}_{(X, Y)}^{\mathrm{gp}}=j_{*} \mathcal{O}_{X \backslash Y}^{\times}$by Lemma 3.3, and so we obtain an injective map

$$
H^{1}\left(X, \mathcal{M}_{(X, Y)}^{\mathrm{gp}}\right) \longrightarrow H^{1}\left(X \backslash Y, \mathcal{O}_{X \backslash Y}^{\times}\right)
$$

from the Leray spectral sequence for $j$. If in fact $R^{1} j_{*} \mathcal{O}_{X \backslash Y}^{\times}=0$, then we would have

$$
H^{1}\left(X, \mathcal{M}_{(X, Y)}^{\mathrm{gp}}\right)=\operatorname{Pic}(X \backslash Y) .
$$

If this is the case, it becomes clear $H^{1}\left(X, \mathcal{M}_{(X, Y)}^{\mathrm{gp}}\right)$ is a natural notion for the logarithmic Picard group. This seems likely to be the case for some natural classes of pairs $Y \subseteq X$. Here is a trivial case:

Lemma 5.9. Let $X$ be a toric variety over an algebraically closed field $k$, and let $D \subseteq X$ be the union of codimension $\geq 1$ toric strata of $X$. Let $Z \subseteq X$ be any closed subset such that $Z$ does not contain any toric stratum of $X$. Then

$$
H^{1}\left(X \backslash Z, \mathcal{M}_{(X, D)}^{\mathrm{gp}}\right)=\operatorname{Pic}(X \backslash(D \cup Z))=0 .
$$


Proof. Since $X \backslash D$ is just an algebraic torus, $\operatorname{Pic}(X \backslash D)=\operatorname{Pic}(X \backslash$ $(D \cup Z))=0$. Since $(5.5)$ gives an injection $H^{1}\left(X \backslash Z, \mathcal{M}_{(X, D)}^{\mathrm{gp}}\right) \rightarrow$ $H^{1}\left(X \backslash(Z \cup D), \mathcal{O}_{X \backslash D}^{\times}\right)$, the result follows. $\quad$ q.e.d.

The situation of interest for us is that $f: \mathcal{X} \rightarrow S=\operatorname{Spec} R$ is a toric degeneration of Calabi-Yau varieties, with singular set $Z$. Then $f$ : $\mathcal{X} \backslash Z \rightarrow S$ is $\log$ smooth, and we then conjecturally have an isomorphism

$$
\operatorname{Pic}\left(\mathcal{X}_{\bar{\eta}} \backslash Z\right) \cong H^{1}\left(\mathcal{X} \backslash Z, \mathcal{M}_{\left(\mathcal{X}, \mathcal{X}_{0}\right)}^{\mathrm{gp}}\right)
$$

Since $Z$ is codimension two, if $\mathcal{X}_{\bar{\eta}}$ is non-singular, we have $\operatorname{Pic}\left(\mathcal{X}_{\bar{\eta}} \backslash Z\right) \cong$ $\operatorname{Pic}\left(\mathcal{X}_{\bar{\eta}}\right)$. In addition, if $\mathcal{M}_{\mathcal{X}_{0}}$ is the induced log structure on $\mathcal{X}_{0}$, there is a natural map $H^{1}\left(\mathcal{X} \backslash Z, \mathcal{M}_{\left(\mathcal{X}, \mathcal{X}_{0}\right)}^{g p}\right) \rightarrow H^{1}\left(\mathcal{X}_{0} \backslash Z, \mathcal{M}_{\mathcal{X}_{0}}^{\text {gp }}\right)$.

This motivates the following conjecture:

Conjecture 5.10. Let $f: \mathcal{X} \rightarrow S$ be a toric degeneration, $X=\mathcal{X}_{0}$. Then if $H^{1}\left(X, \mathcal{O}_{X}\right)=H^{2}\left(X, \mathcal{O}_{X}\right)=0$,

$$
\operatorname{Pic}\left(\mathcal{X}_{\bar{\eta}} \backslash Z\right) \cong H^{1}\left(X \backslash Z, \mathcal{M}_{X}^{\mathrm{gp}}\right) .
$$

If $Z=\emptyset$ and $f$ is normal crossings, then this should follow from representability of the logarithmic Picard functor as proved by Olsson in [40]. This representability probably does not depend on $f$ being normal crossings, but the question is more subtle when $Z \neq \emptyset$ and here we may expect to need some additional hypotheses.

This is meant to be motivation for computing the group $H^{1}(X \backslash$ $\left.Z, \mathcal{M}_{X}^{\mathrm{gp}}\right)$, which we will now do. This in turn serves as motivation for the definition of the log Kähler moduli space in the next section.

We will now begin to set up the technical means for a computation of the logarithmic Picard group in our case. Let $B$ be an integral affine manifold with singularities with a toric polyhedral decomposition $\mathscr{P}$, and let $s$ be open gluing data for $(B, \mathscr{P})$ over an algebraically closed field $k$. Let $X=X_{0}(B, \mathscr{P}, s)$, and suppose there is a log Calabi-Yau space structure on $X$ with log-singular set $Z$. Denote by $\bar{s}$ the associated closed gluing data. Then Definition 2.11 defined the gluing functor $F_{S, \bar{s}}$.

For $\tau \in \mathscr{P}$, let $q_{\tau}: X_{\tau} \rightarrow X$ be the usual natural map, and we will also write here $q_{\tau}$ for the restriction $q_{\tau}: X_{\tau} \backslash q_{\tau}^{-1}(Z) \rightarrow X \backslash Z$. We write $\mathcal{M}_{\tau}:=q_{\tau}^{*} \mathcal{M}_{X \backslash Z}$, the pull-back of the log structure (Definition 3.4).

Lemma 5.11. Let

$$
\mathscr{C}^{k}=\bigoplus_{\substack{\sigma_{0} \rightarrow \ldots \rightarrow \sigma_{k} \\ \sigma_{i} \neq \sigma_{i+1}}} q_{\sigma_{k} *}\left(\mathcal{M}_{\sigma_{k}}^{\mathrm{gp}}\right),
$$


and define a differential $d_{\mathrm{bct}}: \mathscr{C}^{k} \rightarrow \mathscr{C}^{k+1}$ by

$$
\begin{aligned}
\left(d_{\mathrm{bct}}(\alpha)\right)_{\sigma_{0} \rightarrow \cdots \rightarrow \sigma_{k+1}}= & \sum_{i=0}^{k}(-1)^{i} \alpha_{\sigma_{0} \rightarrow \cdots \hat{\sigma}_{i} \rightarrow \cdots \rightarrow \sigma_{k+1}} \\
& +(-1)^{k+1} F_{S, \bar{s}}\left(\sigma_{k} \rightarrow \sigma_{k+1}\right)^{*}\left(\alpha_{\sigma_{0} \rightarrow \cdots \rightarrow \sigma_{k}}\right) .
\end{aligned}
$$

Then $\left(\mathscr{C}^{\bullet}, d_{\mathrm{bct}}\right)$ is a resolution of the sheaf $\mathcal{M}_{X \backslash Z}^{\mathrm{gp}}$.

Proof. Define a map $\mathcal{M}_{X \backslash Z}^{\mathrm{gp}} \rightarrow \mathscr{C}^{0}$ by

$$
\alpha \mapsto\left(q_{\tau}^{*}(\alpha)\right)_{\tau \in \mathscr{P}} .
$$

We need to show $0 \rightarrow \mathcal{M}_{X \backslash Z}^{\mathrm{gp}} \rightarrow \mathscr{C} \bullet$ is exact.

As for any pull-back of log-structures, $\overline{\mathcal{M}}_{\sigma}^{\mathrm{gp}}=q_{\sigma}^{-1}\left(\overline{\mathcal{M}}_{X \backslash Z}^{\mathrm{gp}}\right)$ for each $\sigma \in \mathscr{P}$. Let

$$
\mathcal{I}^{k}=\bigoplus_{\sigma_{0} \rightarrow \cdots \rightarrow \sigma_{k}} q_{\sigma_{k} *} \mathcal{O}_{X_{\sigma_{k}} \backslash q_{\sigma_{k}}^{-1}(Z)}
$$

and

$$
\overline{\mathscr{C}}^{k}=\bigoplus_{\sigma_{0} \rightarrow \cdots \rightarrow \sigma_{k}} q_{\sigma_{k} *} \overline{\mathcal{M}}_{\sigma_{k}}^{\mathrm{gp}}
$$

The differentials are defined similarly to $d_{\text {bct }}$ (though the differential should be written multiplicatively for $\mathcal{I}^{\bullet}$ ). We then get an exact sequence of complexes

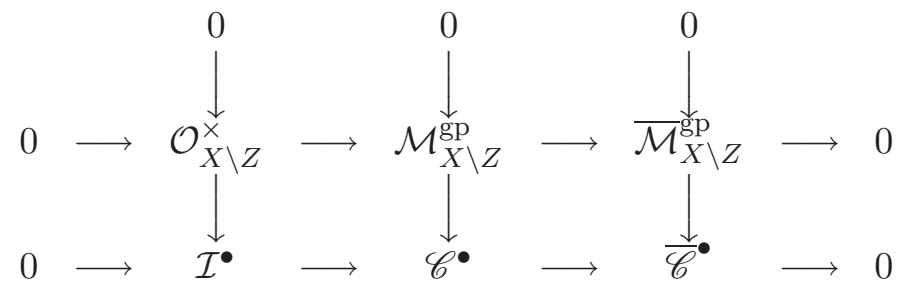

After taking the long exact cohomology sequence, we see the middle complex is exact if the left and right complexes are exact. However, the exactness of these two complexes follows immediately from the methods of $\S$ A.3, Example A.3, as in the proof of Proposition 2.37. q.e.d.

Definition 5.12. For any $\tau \in \mathscr{P}$, we denote by $D_{\tau} \subseteq X_{\tau}$ the toric boundary of $X_{\tau}$.

Lemma 5.13. For any $\tau \in \mathscr{P}$, there is a natural exact sequence

$$
0 \longrightarrow \mathcal{M}_{\left(X_{\tau}, D_{\tau}\right)}^{\mathrm{gp}} \longrightarrow \mathcal{M}_{\tau}^{\mathrm{gp}} \longrightarrow \Lambda_{\tau}^{*} \oplus \mathbb{Z} \longrightarrow 0
$$

on $X_{\tau} \backslash q_{\tau}^{-1}(Z)$. This exact sequence splits, and the splitting is canonical if $\operatorname{dim} \tau=0$. In addition, $H^{1}\left(X_{\tau} \backslash Z, \mathcal{M}_{\tau}^{\mathrm{gp}}\right)=0$ for all $\tau \in \mathscr{P}$. Thus $H^{1}\left(X \backslash Z, \mathcal{M}_{X}^{\mathrm{gp}}\right)=\mathbb{H}^{1}\left(X \backslash Z, \mathscr{C}^{\bullet}\right)=H^{1}\left(\Gamma\left(X \backslash Z, \mathscr{C}^{\bullet}\right)\right)$ by the hypercohomology spectral sequence. 
Proof. First consider the case of a vertex $w \in \mathscr{P}$. We use the construction of $q_{w}$ given in Lemma 2.29. For each $e \in \coprod_{\sigma \in \mathscr{P}_{\max }} \operatorname{Hom}(\{w\}, \sigma)$, we can view $\tilde{\sigma} \subseteq \Lambda_{\mathbb{R}, w}$, with $0=\tilde{w} \in \tilde{\sigma}$ the lift of $w \in \sigma$ distinguished by $e$. We then obtain the closed embedding $\iota_{e}: V_{e} \rightarrow V(\sigma)$, and $q_{w}$ is given on the open subset $V_{e}=\operatorname{Spec} k\left[P_{e}\right]$ of $X_{\omega}$ as $p \circ s_{e}^{-1} \circ \iota_{e}$. Here $P_{e}$ is the face of $P_{\sigma}$ corresponding to $e$. Now the log structure on $X$ pulled back to $V(\sigma)$ is given by a section $f \in \Gamma\left(V(\sigma) \backslash p^{-1}(Z), \mathcal{L} \mathcal{S}_{V(\sigma)}\right)$. In fact this $\log$ structure is given by a collection of charts $P_{\sigma} \rightarrow \mathcal{O}_{U_{i}}$ for an open covering of Zariski open subsets $\left\{U_{i}\right\}$ of $V(\sigma) \backslash p^{-1}(Z)$. The reason for this is that in the proof of Theorem 3.22, giving a section of $\mathcal{F}$ over a Zariski-open subset, gives a chart in the Zariski topology. After further pulling back the $\log$ structure by $s_{e}^{-1}$, we still obtain charts $P_{\sigma} \rightarrow \mathcal{O}_{U_{i}}$ (replacing $U_{i}$ by $s_{e}\left(U_{i}\right)$ ). Finally, the log structure pulled back to $V_{e}$ via $\iota_{e}$ is given by restricting these charts to $V_{e} \cap U_{i}$, yielding $\varphi_{i}: P_{\sigma} \rightarrow \mathcal{O}_{U_{i} \cap V_{e}}$. Such a chart is now of the form

$$
p \mapsto \begin{cases}0 & p \notin P_{e} \\ h_{p} z^{p} & p \in P_{e}\end{cases}
$$

where $P_{e} \ni p \mapsto h_{p} \in \mathcal{O}_{U_{i} \cap V_{e}}^{\times}$is a monoid homomorphism. However, the log structure induced by this chart is in fact isomorphic to the one given by the chart $\varphi_{i}^{\prime}: P_{\sigma} \rightarrow \mathcal{O}_{U_{i} \cap V_{e}}$ given by

$$
p \mapsto \begin{cases}0 & p \notin P_{e} \\ z^{p} & p \in P_{e} .\end{cases}
$$

Explicitly, this isomorphism is given by, for example,

$$
\begin{aligned}
& \left(P_{\sigma} \oplus \mathcal{O}_{V_{e} \cap U_{i}}^{\times}\right) /\left\{\left(p, \varphi_{i}(p)^{-1}\right) \mid p \in \varphi_{i}^{-1}\left(\mathcal{O}_{U_{i} \cap V_{e}}^{\times}\right)\right\} \\
\longrightarrow & \left(P_{\sigma} \oplus \mathcal{O}_{V_{e} \cap U_{i}}^{\times}\right) /\left\{\left(p, \varphi_{i}^{\prime}(p)^{-1}\right) \mid p \in \varphi_{i}^{-1}\left(\mathcal{O}_{U_{i} \cap V_{e}}^{\times}\right)\right\}
\end{aligned}
$$

by $(p, h) \mapsto\left(p, h \cdot h_{\pi(p)}\right)$, where $\pi: P_{\sigma}^{\mathrm{gp}} \rightarrow P_{e}^{\mathrm{gp}}$ is any linear projection and $p \mapsto h_{p}$ has been extended to a homomorphism $P_{e}^{\mathrm{gp}} \rightarrow \mathcal{O}_{U_{i} \cap V_{e}}^{\times}$.

Let $j: \eta \rightarrow X_{w}$ be the inclusion of the generic point $\eta$ of $X_{w}$. Then there is a natural map

$$
\mathcal{M}_{w} \stackrel{\beta}{\longrightarrow} j_{*} j^{*} \overline{\mathcal{M}}_{w}=\mathbb{N} .
$$

Let $\mathcal{M}_{w}^{\prime}=\beta^{-1}(0)$. From the explicit description of charts for $\mathcal{M}_{w}$ above, one sees in fact that if $\alpha: \mathcal{M}_{w} \rightarrow \mathcal{O}_{X_{w}}$ is the structure map, then $\left.\alpha\right|_{\mathcal{M}_{w}^{\prime}}$ is an inclusion of $\mathcal{M}_{w}^{\prime}$ in $\mathcal{O}_{X_{w}}$, and $\alpha\left(\mathcal{M}_{w}^{\prime}\right)=\mathcal{M}_{\left(X_{w}, D_{w}\right)} \subseteq \mathcal{O}_{X_{w}}$. Thus we obtain an exact sequence

$$
0 \rightarrow \mathcal{M}_{\left(X_{w}, D_{w}\right)}^{\mathrm{gp}} \rightarrow \mathcal{M}_{w}^{\mathrm{gp}} \rightarrow \mathbb{Z} \rightarrow 0 .
$$

This can be split canonically via $\mathbb{Z} \ni 1 \mapsto \rho \in \mathcal{M}_{w}^{\text {gp }}$, where $\rho$ is given by the morphism to Spec $k^{\dagger}$.

If $\tau$ is arbitrary, we can choose any $e: w \rightarrow \tau$, and then use $q_{\tau}=$ $q_{w} \circ F_{S, \bar{s}}(e)$ to obtain charts for $\mathcal{M}_{\tau}$ on open subsets of $X_{\tau}$. Now if 
$j: \eta \rightarrow X_{\tau}$ is the inclusion of the generic point, then by the construction of the dual intersection complex in $\S 4, j_{*} j^{*} \overline{\mathcal{M}_{\tau}}$ can be identified as the constant monoid sheaf $\operatorname{Hom}\left(\check{P}_{\tau}, \mathbb{N}\right) \subseteq \Lambda_{\tau}^{*} \oplus \mathbb{Z}$. (See Definition 2.1.) Thus $\left(j_{*} j^{*} \overline{\mathcal{M}_{\tau}}\right)^{\text {gp }}$ can be identified with $\Lambda_{\tau}^{*} \oplus \mathbb{Z}$, though this identification is not completely canonical, as it depends on the lifting $\tilde{\tau}$. The same argument as before now shows we have an exact sequence

$$
0 \longrightarrow \mathcal{M}_{\left(X_{\tau}, D_{\tau}\right)}^{\mathrm{gp}} \longrightarrow \mathcal{M}_{\tau}^{\mathrm{gp}} \stackrel{\beta}{\longrightarrow} \Lambda_{\tau}^{*} \oplus \mathbb{Z} \longrightarrow 0
$$

on $X_{\tau} \backslash q_{\tau}^{-1}(Z)$.

By Lemma $5.9, H^{1}\left(X_{\tau} \backslash q_{\tau}^{-1}(Z), \mathcal{M}_{\left(X_{\tau}, D_{\tau}\right)}^{\mathrm{gp}}\right)=0$. In addition, since $H^{1}(Y, \mathbb{Z})=0$ in the étale topology for any normal variety $Y,([\mathbf{3}]$, IX.3.6), we see that $H^{1}\left(X_{\tau} \backslash q_{\tau}^{-1}(Z), \mathcal{M}_{\tau}^{\text {gp }}\right)=0$. The last statement of the Lemma then follows from Lemma 5.11 and the hypercohomology spectral sequence.

The only remaining statement to show is that the exact sequence of the Lemma splits. But $\operatorname{Ext}^{1}\left(\mathbb{Z}, \mathcal{M}_{\left(X_{\tau}, D_{\tau}\right)}^{\mathrm{gp}}\right)=H^{1}\left(X_{\tau} \backslash q_{\tau}^{-1}(Z)\right.$, $\left.\mathcal{M}_{\left(X_{\tau}, D_{\tau}\right)}^{\mathrm{gp}}\right)=0$, so the extension class must be trivial. There is no canonical splitting, however.

q.e.d.

The moral of this calculation is that while there is moduli of log structures on $V(\sigma)$, these all become a standard log structure once restricted to irreducible components.

We now want to compute $H^{1}(\Gamma(X \backslash Z, \mathscr{C} \bullet))$.

Definition 5.14. Letting

$$
Q^{k}=\Gamma\left(X \backslash Z, \mathscr{C}^{k}\right) / \Gamma\left(X \backslash Z, \mathcal{I}^{k}\right),
$$

where $\mathcal{I}^{k}=\bigoplus_{\sigma_{0} \rightarrow \cdots \rightarrow \sigma_{k}} q_{\sigma_{k} *} \mathcal{O}_{X_{\sigma_{k}} \backslash q_{\sigma_{k}}^{-1}(Z)}$ is as in Lemma 5.11, we obtain an exact sequence of complexes

$$
0 \rightarrow \Gamma\left(X \backslash Z, \mathcal{I}^{\bullet}\right) \rightarrow \Gamma\left(X \backslash Z, \mathscr{C}^{\bullet}\right) \rightarrow Q^{\bullet} \rightarrow 0 .
$$

To think about elements of $Q^{k}$, we first use the sequence

$$
0 \rightarrow \mathcal{O}_{X_{\tau} \backslash q_{\tau}^{-1}(Z)}^{\times} \rightarrow \mathcal{M}_{\tau}^{\mathrm{gp}} \rightarrow \overline{\mathcal{M}}_{\tau}^{\mathrm{gp}} \rightarrow 0
$$

to see that

$$
\begin{aligned}
\Gamma\left(X_{\tau} \backslash q_{\tau}^{-1}(Z), \mathcal{M}_{\tau}^{\mathrm{gp}}\right) / \Gamma\left(X_{\tau} \backslash q_{\tau}^{-1}(Z), \mathcal{O}_{X_{\tau}}^{\times}\right) \\
\cong \operatorname{ker}\left(H^{0}\left(X_{\tau} \backslash q_{\tau}^{-1}(Z), \overline{\mathcal{M}}_{\tau}^{\mathrm{gp}}\right) \rightarrow \operatorname{Pic}\left(X_{\tau} \backslash q_{\tau}^{-1}(Z)\right)\right) .
\end{aligned}
$$

Thus we need a way to think of elements of $H^{0}\left(X_{\tau} \backslash q_{\tau}^{-1}(Z), \overline{\mathcal{M}}_{\tau}^{\mathrm{gp}}\right)$.

Lemma 5.15. Let $\tau \in \mathscr{P}$, and choose $e: v \rightarrow \tau$. Let $\tau_{e}$ be the cone of $\Sigma_{v}$ corresponding to $e$, with

$$
\tau_{e}^{-1} \Sigma_{v}=\left\{K+\mathbb{R} \tau_{e} \mid K \in \Sigma_{v} \text { with } \tau_{e} \subseteq K\right\} .
$$


Then there is a canonical isomorphism between

$$
H^{0}\left(X_{\tau} \backslash q_{\tau}^{-1}(Z), \overline{\mathcal{M}}_{\tau}^{\mathrm{gp}}\right)
$$

and the group

$$
\begin{aligned}
\operatorname{PA}(e) & =\left\{\begin{array}{l|l}
\lambda: \Lambda_{\mathbb{R}, v} \rightarrow \mathbb{R} & \begin{array}{l}
\lambda \text { is piecewise affine with respect to } \\
\tau_{e}^{-1} \Sigma_{v} \text { and takes integer values on } \Lambda_{v}
\end{array}
\end{array}\right\} \\
& =\mathcal{P} \mathcal{L}_{\mathscr{P}}(B, \mathbb{Z})_{y}
\end{aligned}
$$

for $y \in \tau$ in the interior of the edge $e$ of $\operatorname{Bar}(\mathscr{P})$.

Proof. Let $\lambda \in H^{0}\left(X_{\tau} \backslash q_{\tau}^{-1}(Z), \overline{\mathcal{M}}_{\tau}^{\mathrm{gp}}\right)$. Since $\overline{\mathcal{M}}_{\tau}^{\mathrm{gp}}=q_{\tau}^{-1}\left(\overline{\mathcal{M}}_{X}^{\mathrm{gp}}\right)$, we have stalks $\left(\overline{\mathcal{M}}_{\tau}^{\mathrm{gp}}\right)_{\bar{x}}=\overline{\mathcal{M}}_{X, q_{\tau}(\bar{x})}^{\text {gp }}$. Now any $f: \tau \rightarrow \sigma$ determines a toric stratum $F_{S, \bar{s}}\left(X_{\sigma}\right) \subseteq X_{\tau}$ with generic point $\eta_{\sigma}$, and thus $\lambda$ determines a stalk $\lambda_{\sigma} \in \overline{\mathcal{M}}_{X, q_{\tau}\left(\bar{\eta}_{\sigma}\right)}^{\mathrm{gp}}$. On the other hand, the construction of the dual intersection complex identifies $\tilde{\sigma}$ canonically with a polytope in an affine hyperplane in $\operatorname{Hom}\left(\overline{\mathcal{M}}_{X, q_{\tau}\left(\bar{\eta}_{\sigma}\right)}^{\mathrm{gp}}, \mathbb{R}\right)$, and $\lambda_{\sigma}$ can be viewed as a linear functional on this latter vector space. By restricting $\lambda_{\sigma}$ to $\tilde{\sigma}$, we obtain a canonically defined linear function $\lambda_{\sigma}: \tilde{\sigma} \rightarrow \mathbb{R}$. The morphism $e$ also determines a lift $\tilde{v} \in \tilde{\sigma}$. By identifying the tangent wedge to $\tilde{\sigma}$ at $\tilde{v}$, based at $\tilde{v}$, with the corresponding cone $K$ in the fan $\Sigma_{v}$, we obtain just as well a canonically defined affine linear function $\lambda_{\sigma}^{\prime}: K \rightarrow \mathbb{R}$ (so that $\left.\lambda_{\sigma}^{\prime}(0)=\lambda_{\sigma}(\tilde{v})\right)$. Note that $\tau_{e}$ is a face of $K$. Then $\lambda_{\sigma}^{\prime}$ extends to a map $\lambda_{\sigma}^{\prime}: K+\mathbb{R} \tau_{e} \rightarrow \mathbb{R}$ which is affine linear and takes integer values on $\Lambda_{v} \cap\left(K+\mathbb{R} \tau_{e}\right)$. The collection of such $\lambda_{\sigma}^{\prime}$ defines a map $\lambda: \Lambda_{\mathbb{R}, v} \rightarrow \mathbb{R}$ which is piecewise affine as desired. The fact that $\lambda$ is continuous comes from the fact that if we have $e_{i}: \omega \rightarrow \sigma_{i}, i=1,2$, the images of $\lambda_{\sigma_{1}}$ and $\lambda_{\sigma_{2}}$ under cospecialization maps

$$
\overline{\mathcal{M}}_{\tau, \bar{\eta}_{\sigma_{1}}}^{\mathrm{gp}} \rightarrow \overline{\mathcal{M}}_{\tau, \bar{\eta}_{\omega}}^{\mathrm{gp}} \leftarrow \overline{\mathcal{M}}_{\tau, \bar{\eta}_{\sigma_{2}}}^{\mathrm{gp}}
$$

coincide with $\lambda_{\omega}$.

Reversing this procedure, given a piecewise affine map $\lambda: \Lambda_{\mathbb{R}, v} \rightarrow \mathbb{R}$, one obtains stalks $\lambda_{\sigma}$ of $\overline{\mathcal{M}}_{\tau}^{\text {gp }}$ which agree under cospecialization, and hence give rise to a section of $\overline{\mathcal{M}}_{\tau}^{\mathrm{gp}}$. The last equality follows from the definition of $\mathcal{P} \mathcal{L}_{\mathscr{P}}(B, \mathbb{Z})$.

q.e.d.

Remark 5.16. Of course $H^{0}\left(X_{\tau} \backslash q_{\tau}^{-1}(Z), \overline{\mathcal{M}}_{\tau}^{\text {gp }}\right)$ is independent of the choice of $e$, so if we have $e_{i}: v_{i} \rightarrow \tau$, then the resulting canonical isomorphism $\phi: \mathrm{PA}\left(e_{1}\right) \rightarrow \mathrm{PA}\left(e_{2}\right)$ is easily described. For any maximal cone $K_{1}$ of $\tau_{e_{1}}^{-1} \Sigma_{v_{1}}$ corresponding to $f: \tau \rightarrow \sigma \in \mathscr{P}_{\max }$, an element $\lambda \in \operatorname{PA}\left(e_{1}\right)$ restricted to $K_{1}$ is given by an element $\lambda_{K_{1}} \in \mathcal{A} f f(B, \mathbb{Z})_{v_{1}}$. Then $\phi(\lambda)$ restricted to the corresponding cone $K_{2}$ of $\tau_{e_{2}}^{-1} \Sigma_{v_{2}}$ is given by parallel transport of $\lambda_{K_{1}}$ to $\lambda_{K_{2}} \in \mathcal{A f f}(B, \mathbb{Z})_{v_{2}}$ by first following the edge of $\operatorname{Bar}(\mathscr{P})$ given by $f \circ e_{1}$, and then following the edge $f \circ e_{2}$ to arrive at $v_{2}$. This construction can be viewed as dual to the construction of the map $\phi$ of Construction 2.15. 
Remark 5.17. By Lemma 5.13, we always have a subsheaf $\overline{\mathcal{M}}_{\left(X_{\tau}, D_{\tau}\right)}^{\mathrm{gp}} \subseteq \overline{\mathcal{M}}_{\tau}$. We can interpret an element $\lambda \in \Gamma\left(X_{\tau} \backslash q_{\tau}^{-1}(Z)\right.$, $\left.\overline{\mathcal{M}}_{\left(X_{\tau}, D_{\tau}\right)}^{\mathrm{gp}}\right)$ as a piecewise linear function on the fan $\Sigma_{\tau}$ in a slightly different way. Such a section can be interpreted as a Cartier divisor on $X_{\tau}$ supported on $D_{\tau}$, and as such gives a well-defined piecewise linear function $\lambda$ on the fan $\Sigma_{\tau}$ taking integer values on $\mathcal{Q}_{\tau} \subseteq \mathcal{Q}_{\tau, \mathbb{R}}$, by the standard correspondence between toric Cartier divisors and piecewise linear functions (see Remark 0.1). Given $e: v \rightarrow \sigma$, we can compose $\lambda$ with the projection $\Lambda_{\mathbb{R}, v} \rightarrow \mathcal{Q}_{\tau, \mathbb{R}}$, to obtain a piecewise linear function on $\tau_{e}^{-1} \Sigma_{v}$. It is easy to check this coincides with the piecewise affine function constructed in Lemma 5.15.

Now in general, the boundary map

$$
H^{0}\left(X_{\tau} \backslash q_{\tau}^{-1}(Z), \overline{\mathcal{M}}_{\left(X_{\tau}, D_{\tau}\right)}^{\mathrm{gp}}\right) \rightarrow \operatorname{Pic}\left(X_{\tau} \backslash q_{\tau}^{-1}(Z)\right)
$$

induced by the standard short exact sequence takes a Cartier divisor with support on $D_{\tau}$ to the corresponding line bundle on $X_{\tau} \backslash q_{\tau}^{-1}(Z)$. Thus, in particular, if $\lambda \in H^{0}\left(X_{\tau} \backslash q_{\tau}^{-1}(Z), \overline{\mathcal{M}}_{\tau}^{\mathrm{gp}}\right)$ is induced by a linear function on $\Sigma_{\tau}$, then the corresponding Cartier divisor is principal and $\lambda$ lifts to an element of $H^{0}\left(X_{\tau} \backslash q_{\tau}^{-1}(Z), \mathcal{M}_{\tau}^{\mathrm{gp}}\right)$.

Lemma 5.18. Let $W_{\sigma_{0} \rightarrow \cdots \rightarrow \sigma_{p}}$ denote the connected component of $W_{\sigma_{0} \cdots \sigma_{p}}$ corresponding to the $p$-dimensional simplex of $\operatorname{Bar}(\mathscr{P})$ with edges $\sigma_{i} \rightarrow \sigma_{i+1}$ (see Lemma 2.9). Then there is a canonical map

$$
\begin{aligned}
& \Gamma\left(W_{\sigma_{0} \rightarrow \cdots \rightarrow \sigma_{p}}, \mathcal{A} f f(B, \mathbb{Z})\right) \longrightarrow \\
& \Gamma\left(X_{\sigma_{p}} \backslash q_{\sigma_{p}}^{-1}(Z), \mathcal{M}_{\sigma_{p}}^{\mathrm{gp}}\right) / \Gamma\left(X_{\sigma_{p}} \backslash q_{\sigma_{p}}^{-1}(Z), \mathcal{O}_{X_{\sigma_{p}}}^{\times}\right) .
\end{aligned}
$$

This induces an injective morphism of complexes

$$
\check{C}^{\bullet}(\mathscr{W}, \mathcal{A f f}(B, \mathbb{Z})) \rightarrow Q^{\bullet}
$$

where the former complex denotes the Čech complex for $\mathcal{A} f f(B, \mathbb{Z})$ with respect to the open cover $\mathscr{W}$.

Proof. Consider first $w \in \mathscr{P}$ a vertex. By Lemma 5.13, there is a canonical isomorphism

$$
\Gamma\left(X_{w} \backslash q_{w}^{-1}(Z), \mathcal{M}_{w}^{\mathrm{gp}}\right) \cong \Gamma\left(X_{w} \backslash q_{w}^{-1}(Z), \mathcal{M}_{\left(X_{w}, D_{w}\right)}^{\mathrm{gp}}\right) \oplus \mathbb{Z} \rho .
$$

Now $\mathcal{M}_{\left(X_{w}, D_{w}\right)}^{\mathrm{gp}}$ is the sheaf of rational functions on $X_{w}$ with zeros and poles only along $D_{w}$. Thus, since $q_{w}^{-1}(Z) \subseteq D_{w}$, elements of $\Gamma\left(X_{w} \backslash q_{w}^{-1}(Z), \mathcal{M}_{\left(X_{w}, D_{w}\right)}^{\mathrm{gp}}\right) / \Gamma\left(X_{w} \backslash q_{w}^{-1}(Z), \mathcal{O}_{X_{w}}^{\times}\right)$correspond to principal Cartier divisors on $X_{w}$ with support on $D_{w}$, and hence by Remark 5.17, correspond to linear functions on $\Lambda_{w}$. In addition, $\rho \in$ $\Gamma\left(X_{w} \backslash q_{w}^{-1}(Z), \mathcal{M}_{w}^{\mathrm{gp}}\right)$ corresponds to the constant function taking the value 1 on $\Lambda_{w}$. Thus $\Gamma\left(X_{w} \backslash q_{w}^{-1}(Z), \mathcal{M}_{w}^{\mathrm{gp}}\right) / \Gamma\left(X_{w} \backslash q_{w}^{-1}(Z), \mathcal{O}_{X_{w}}^{\times}\right)$is isomorphic to the subgroup of $\mathrm{PA}(\mathrm{id}: w \rightarrow w)=\mathcal{P} \mathcal{L}_{\mathscr{P}}(B, \mathbb{Z})_{w}$ consisting 
of affine linear functions. This gives a canonical isomorphism

$$
\begin{aligned}
& \mathcal{A} f f(B, \mathbb{Z})_{w}=\Gamma\left(W_{w}, \mathcal{A f f}(B, \mathbb{Z})\right) \longrightarrow \longrightarrow \\
& \Gamma\left(X_{w} \backslash q_{w}^{-1}(Z), \mathcal{M}_{w}^{\mathrm{gp}}\right) / \Gamma\left(X_{w} \backslash q_{w}^{-1}(Z), \mathcal{O}_{X_{w}}^{\times}\right) .
\end{aligned}
$$

We next consider the general case. Let $\sigma_{0} \rightarrow \cdots \rightarrow \sigma_{p}$ be a chain of morphisms defining some element of $\operatorname{Bar}(\mathscr{P})$. Write $W=W_{\sigma_{0} \rightarrow \cdots \rightarrow \sigma_{p}}$. Pick a vertex $w \in \sigma_{0}$ and a morphism $w \rightarrow \sigma_{0}$. Then $w \in \bar{W}$, and by parallel transport from a basepoint inside $W$ to $w$ we can identify $\Gamma(W, \mathcal{A} f f(B, \mathbb{Z}))$ canonically with a subgroup of $\mathcal{A} f f(B, \mathbb{Z})_{w}$ invariant under monodromy in $W$. We then define a map as the composition

$$
\begin{aligned}
\Gamma(W, \mathcal{A} f f & (B, \mathbb{Z})) \hookrightarrow \mathcal{A f f}(B, \mathbb{Z})_{w}=\Gamma\left(W_{w}, \mathcal{A} f f(B, \mathbb{Z})\right) \longrightarrow \\
& \frac{\Gamma\left(X_{w} \backslash q_{w}^{-1}(Z), \mathcal{M}_{w}^{\mathrm{gp}}\right)}{\Gamma\left(X_{w} \backslash q_{w}^{-1}(Z), \mathcal{O}_{X_{w}}^{\times}\right)} \stackrel{F_{S, \bar{s}}\left(w \rightarrow \sigma_{p}\right)^{*}}{\longrightarrow} \frac{\Gamma\left(X_{\sigma_{p}} \backslash q_{\sigma_{p}}^{-1}(Z), \mathcal{M}_{\sigma_{p}}^{\mathrm{gp}}\right)}{\Gamma\left(X_{\sigma_{p}} \backslash q_{\sigma_{p}}^{-1}(Z), \mathcal{O}_{X_{\sigma_{p}}}^{\times}\right)} .
\end{aligned}
$$

We need to show this map is independent of the choice of $w$, so choose $e_{1}: w_{1} \rightarrow \sigma_{0}$ and $e_{2}: w_{2} \rightarrow \sigma_{0}$. Now if we choose some $\sigma_{p} \rightarrow \sigma \in \mathscr{P}_{\max }$, we can identify $\mathcal{A} f f(B, \mathbb{Z})_{w_{1}}$ with $\mathcal{A} f f(B, \mathbb{Z})_{w_{2}}$ via parallel transport $\psi$ from $w_{1}$ into $\operatorname{Int}(\sigma)$ to $w_{2}$. On the other hand, let $f_{i}$ be the composition $w_{i} \stackrel{e_{i}}{\longrightarrow} \sigma_{0} \longrightarrow \sigma_{p}$. Then we have the canonical isomorphism $\phi: \operatorname{PA}\left(f_{1}\right) \rightarrow$ $\mathrm{PA}\left(f_{2}\right)$ given by Remark 5.16. This gives a (not necessarily commutative!) diagram

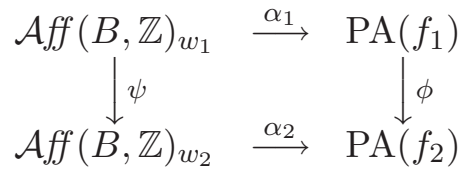

The second vertical map is defined on each maximal cone of $\left(\sigma_{p}\right)_{f_{i}}^{-1} \Sigma_{w_{i}}$ corresponding to $\sigma_{p} \rightarrow \sigma^{\prime} \in \mathscr{P}_{\max }$ by parallel transport from $\mathcal{A} f f(B, \mathbb{Z})_{w_{1}}$ to $\mathcal{A f f}(B, \mathbb{Z})_{w_{2}}$ through $\sigma^{\prime}$. Thus if the parallel transport of $\lambda \in \mathcal{A f f}(B, \mathbb{Z})_{w_{1}}$ to $\mathcal{A} f f(B, \mathbb{Z})_{w_{2}}$ is independent of the choice of $\sigma_{p} \rightarrow \sigma^{\prime}$, then $\alpha_{2}(\psi(\lambda))=\phi\left(\alpha_{1}(\lambda)\right)$. This holds in particular if $\lambda \in \Gamma(W, \mathcal{A} f f(B, \mathbb{Z})) \subseteq \mathcal{A} f f(B, \mathbb{Z})_{w_{i}}$. This shows well-definedness.

Finally, it is easy to check this is sufficiently canonically defined so as to give a map of complexes.

q.e.d.

We are now in a position to carry out the calculation of $H^{1}(X \backslash$ $\left.Z, \mathcal{M}_{X}^{\mathrm{gp}}\right)$ in a way which is formally the same as the calculation of the moduli of log Calabi-Yau spaces of $\S 5.1$. However, this construction will be completely dual.

Theorem 5.19. Let $(B, \mathscr{P})$ be positive and simple, and $s$ lifted gluing data determining a log Calabi-Yau space $X^{\dagger}=X_{0}(B, \mathscr{P}, s)^{\dagger}$ by Theorem 5.2, (2). Then there is an exact sequence

$$
\begin{aligned}
0 & \rightarrow H^{0}\left(B, k^{\times}\right) \rightarrow H^{0}\left(X \backslash Z, \mathcal{M}_{X}^{\mathrm{gp}}\right) \rightarrow H^{0}(B, \mathcal{A} f f(B, \mathbb{Z})) \\
& \rightarrow H^{1}\left(B, k^{\times}\right) \rightarrow H^{1}\left(X \backslash Z, \mathcal{M}_{X}^{\mathrm{gp}}\right) \rightarrow H^{1}\left(Q^{\bullet}\right) \rightarrow H^{2}\left(B, k^{\times}\right)
\end{aligned}
$$


with an injection $H^{1}(B, \mathcal{A} f f(B, \mathbb{Z})) \hookrightarrow H^{1}\left(Q^{\bullet}\right)$ which is an isomorphism when tensored with $\mathbb{Q}$. Furthermore, if $\Delta(\tau)$ of Definition 1.60 is a standard simplex for each $\tau \in \mathscr{P}$ with $\operatorname{dim} \tau \neq 0, \operatorname{dim} B$, rather than just an elementary simplex, then $H^{1}\left(Q^{\bullet}\right)=H^{1}(B, \mathcal{A} f f(B, \mathbb{Z}))$. This holds in particular if $\operatorname{dim} B \leq 3$.

Proof.

Step 1. $H^{0}\left(X_{\tau} \backslash q_{\tau}^{-1}(Z), \mathcal{O}_{X_{\tau}}^{\times}\right)=k^{\times}$. Thus, in particular, $\Gamma(X \backslash$ $\left.Z, \mathcal{I}^{\bullet}\right)$ is the simplicial cochain complex with respect to the triangulation $\operatorname{Bar}(\mathscr{P})$ with coefficients in $k^{\times}$, and $H^{i}\left(\Gamma\left(X \backslash Z, \mathcal{I}^{\bullet}\right)\right)=H^{i}\left(B, k^{\times}\right)$.

Proof of Step 1. If $\alpha \in H^{0}\left(X_{\tau} \backslash q_{\tau}^{-1}(Z), \mathcal{O}_{X_{\tau}}^{\times}\right)$, then the divisor of zeros and poles of $\alpha$ is a divisor supported on $q_{\tau}^{-1}(Z)$. But then by the linear independence statement of Corollary 5.8, $\alpha$ must be constant.

Step 2. Let $\check{C} \bullet(\mathscr{W}, \mathcal{A f f}(B, \mathbb{Z})) \rightarrow Q^{\bullet}$ be the inclusion of complexes of Lemma 5.18. Then $H^{0}\left(Q^{\bullet} / \check{C}^{\bullet}(\mathscr{W}, \mathcal{A} f f(B, \mathbb{Z}))\right)=0$. Thus in particular $H^{0}\left(Q^{\bullet}\right)=H^{0}(\mathscr{W}, \mathcal{A} f f(B, \mathbb{Z}))$ and

$$
H^{1}(\mathscr{W}, \mathcal{A} f f(B, \mathbb{Z})) \rightarrow H^{1}\left(Q^{\bullet}\right)
$$

is injective.

Proof of Step 2. To give an element of $H^{0}\left(Q^{\bullet} / \check{C}(\mathscr{W}, \mathcal{A} f f(B, \mathbb{Z}))\right)$ we give for each $\tau \in \mathscr{P}$ an element

$$
\lambda_{\tau} \in \Gamma\left(X_{\tau} \backslash q_{\tau}^{-1}(Z), \mathcal{M}_{\tau}^{\mathrm{gp}}\right) / k^{\times}
$$

defined modulo $\Gamma\left(W_{\tau}, \mathcal{A f f}(B, \mathbb{Z})\right)$, and such that for $e: \tau_{0} \rightarrow \tau_{1}$,

$$
F_{S, \bar{s}}(e)^{*}\left(\lambda_{\tau_{0}}\right)=\lambda_{\tau_{1}} \bmod \Gamma\left(W_{e}, \mathcal{A} f f(B, \mathbb{Z})\right) .
$$

But for a vertex $v$, in fact

$$
\Gamma\left(X_{v} \backslash q_{v}^{-1}(Z), \mathcal{M}_{v}^{\mathrm{gp}}\right) / k^{\times}=\Gamma\left(W_{v}, \mathcal{A f f}(B, \mathbb{Z})\right),
$$

as we saw in the proof of Lemma 5.18, so we can take $\lambda_{v}=0$, and so for fixed $\tau$ and any $e: v \rightarrow \tau, \lambda_{\tau} \in \Gamma\left(W_{e}, \mathcal{A f f}(B, \mathbb{Z})\right)=\Gamma\left(W_{v}, \mathcal{A f f}(B, \mathbb{Z})\right)=$ $\mathcal{A} f f(B, \mathbb{Z})_{v}$. Now the restriction map $\Gamma\left(W_{\tau}, \mathcal{A} f f(B, \mathbb{Z})\right) \rightarrow \mathcal{A f f}(B, \mathbb{Z})_{v}$ is injective, and if $\lambda_{\tau}$ is not in the image of this map, it is because $\lambda_{\tau}$ is not monodromy invariant with respect to some loop determined by $e_{i}: v_{i} \rightarrow \tau, i=1,2$ and $f_{j}: \tau \rightarrow \sigma_{j} \in \mathscr{P}_{\max }, j=1,2$, passing from $v_{1}$ into the interior of $\sigma_{1}$ to $v_{2}$ into the interior of $\sigma_{2}$ to $v_{1}$. Thus parallel transporting $\lambda_{\tau} \in \mathcal{A f f}(B, \mathbb{Z})_{v_{1}}$ from $v_{1}$ to $v_{2}$ through $\sigma_{1}$ and $\sigma_{2}$ gives different functions. Thus $\lambda_{\tau} \in \mathrm{PA}\left(e_{1}\right)$ is an affine linear function since $\lambda_{\tau} \in \Gamma\left(W_{e_{1}}, \mathcal{A f f}(B, \mathbb{Z})\right)$, but as an element in $\mathrm{PA}\left(e_{2}\right)$, it is no longer affine linear but only piecewise affine. This contradicts $\lambda_{\tau} \in$ $\Gamma\left(W_{e_{2}}, \mathcal{A} f f(B, \mathbb{Z})\right)$. Thus $\lambda_{\tau} \in \Gamma\left(W_{\tau}, \mathcal{A} f f(B, \mathbb{Z})\right)$, so we can take $\lambda_{\tau}=0$. Thus $H^{0}\left(Q^{\bullet} / \check{C}^{\bullet}(\mathscr{W}, \mathcal{A f f}(B, \mathbb{Z}))\right)=0$.

Step 3. Let $\tau \in \mathscr{P}$ and $\lambda \in \Gamma\left(X_{\tau} \backslash q_{\tau}^{-1}(Z), \overline{\mathcal{M}}_{\tau}^{\mathrm{gp}}\right)$. For any $e: v \rightarrow \tau$, denote by $\lambda^{e}$ the corresponding element of $\mathrm{PA}(e)$ (Lemma 5.15). Suppose furthermore we have made choices of $d_{\omega}$ 's and $\check{d}_{\rho}$ 's as in $\S 1.5$. Then if 
$f: \tau \rightarrow \rho$, with codim $\rho=1$, we obtain $g_{\rho}^{ \pm}: \rho \rightarrow \sigma_{\rho}^{ \pm}$, defining maximal cones $K^{ \pm}$of $\tau_{e}^{-1} \Sigma_{v}$, and $\lambda^{e \pm} \in \mathcal{A f f}(B, \mathbb{Z})_{v}$, the restriction of $\lambda^{e}$ to $K^{ \pm}$. Then define $\check{D}(\lambda, e, f) \in \mathbb{Z}$ so that

$$
\check{D}(\lambda, e, f) \check{d}_{\rho}=\lambda^{e-}-\lambda^{e+} .
$$

Now let $\left(\lambda_{e}\right)_{e: \tau_{0} \rightarrow \tau_{1}} \in Q^{1}$ be a 1-cocycle for the complex $Q^{\bullet}$, with $\lambda_{e} \in$ $\Gamma\left(X_{\tau_{1}} \backslash q_{\tau_{1}}^{-1}(Z), \mathcal{M}_{\tau_{1}}^{\mathrm{gp}}\right) / k^{\times} \subseteq \Gamma\left(X_{\tau_{1}} \backslash q_{\tau_{1}}^{-1}(Z), \overline{\mathcal{M}}_{\tau_{1}}^{\mathrm{gp}}\right)$ for $e: \tau_{0} \rightarrow \tau_{1}$. Then $\left(\lambda_{e}\right)$ satisfies a condition dual to the Condition (LC), namely: Let $\rho \in \mathscr{P}$ be a codimension one cell, $v_{1}, v_{2}$ vertices, $\tau \in \mathscr{P}$ with a diagram

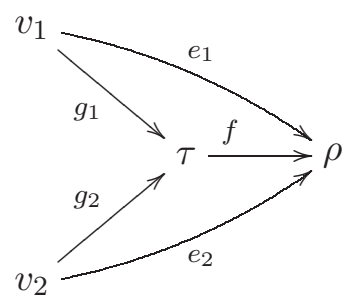

Then if $T_{e_{1} e_{2}}^{\rho}=0$, we must have

$$
\check{D}\left(\lambda_{g_{1}}, g_{1}, f\right)=\check{D}\left(\lambda_{g_{2}}, g_{2}, f\right) .
$$

Proof of Step 3. First consider the case that $v_{1}$ and $v_{2}$ are endpoints of a one-dimensional $\omega \in \mathscr{P}$, so that we have

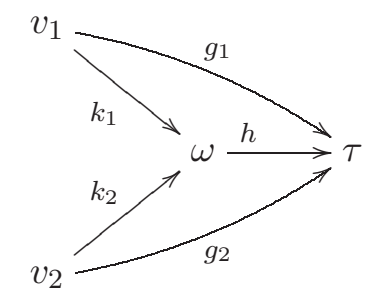

Then because $\left(\lambda_{e}\right)$ is a cocycle, we have

$$
\lambda_{g_{i}}=\lambda_{h}+F_{S, \bar{s}}(h)^{*}\left(\lambda_{k_{i}}\right), \quad i=1,2 .
$$

We will first show that if $T_{e_{1} e_{2}}^{\rho}=0$, then $\check{D}\left(F_{S, \bar{s}}(h)^{*}\left(\lambda_{k_{i}}\right), g_{i}, f\right)=0$ for $i=1,2$. First note that $\check{D}\left(F_{S, \bar{s}}(h)^{*}\left(\lambda_{k_{i}}\right), g_{i}, f\right)=\check{D}\left(\lambda_{k_{i}}, k_{i}, f \circ h\right)$. By Lemma 5.13, we have a splitting $\mathcal{M}_{\omega}^{\mathrm{gp}}=\mathcal{M}_{\left(X_{\omega}, D_{\omega}\right)}^{\mathrm{gp}} \oplus\left(\Lambda_{\omega}^{*} \oplus \mathbb{Z} \rho\right)$. To make this splitting more explicit, choose an element $t_{i} \in \Gamma\left(X_{v_{i}}, \mathcal{M}_{\left(X_{v_{i}}, D_{v_{i}}\right)}^{\mathrm{gp}}\right)$ which vanishes exactly once along the stratum $X_{\omega} \subseteq X_{v_{i}}$. This restricts to an element $t_{i} \in \Gamma\left(X_{\omega} \backslash q_{\omega}^{-1}(Z), \mathcal{M}_{\omega}^{\mathrm{gp}}\right)$, and we can then write $\mathcal{M}_{\omega}^{\mathrm{gp}}=$ $\mathcal{M}_{\left(X_{\omega}, D_{\omega}\right)}^{\mathrm{gp}} \oplus\left(\mathbb{Z} t_{i} \oplus \mathbb{Z} \rho\right)$. Identifying $t_{i}$ and $\rho$ with their images in $\overline{\mathcal{M}}_{\omega}^{\mathrm{gp}}$, we can then write $\lambda_{k_{i}}=u_{i}+a_{i} t_{i}+b_{i} \rho$ for $u_{i} \in \Gamma\left(X_{\omega} \backslash q_{\omega}^{-1}(Z), \overline{\mathcal{M}}_{\left(X_{\omega}, D_{\omega}\right)}^{\mathrm{gp}}\right)$ and $a_{i}, b_{i} \in \mathbb{Z}$. By definition of $Q^{\bullet}, \lambda_{k_{i}}$ is in the image of the map $\Gamma\left(X_{\omega} \backslash q_{\omega}^{-1}(Z), \mathcal{M}_{\omega}^{\mathrm{gp}}\right) \rightarrow \Gamma\left(X_{\omega} \backslash q_{\omega}^{-1}(Z), \overline{\mathcal{M}}_{\omega}^{\mathrm{gp}}\right)$, and since $t_{i}$ and $\rho$ are already in the image of this map, so is $u_{i}$, and so $u_{i}$ maps to zero in 
$\operatorname{Pic}\left(X_{\omega} \backslash q_{\omega}^{-1}(Z)\right)$. Then by construction, if we view $t_{i}, \rho \in \mathrm{PA}\left(k_{i}\right), t_{i}$ is a linear function and $\rho$ is constant, so

$$
\check{D}\left(\lambda_{k_{i}}, k_{i}, f \circ h\right)=\check{D}\left(u_{i}, k_{i}, f \circ h\right) .
$$

Now $u_{i}$ determines a Cartier divisor $C_{i}$ on $X_{\omega}$ supported on $D_{\omega}$, and as an element of $\mathrm{PA}\left(k_{i}\right)$, by Remark 5.17, $u_{i}$ is just the pull-back of the corresponding piecewise linear function on $\Sigma_{\omega}$. Furthermore, $\check{D}\left(u_{i}, k_{i}, f \circ h\right)$ can be interpreted as an element of $\operatorname{Pic}\left(X_{\rho}\right)=\mathbb{Z}$ (see [38], Lemma 2.11 ), and as such is the pull-back of $C_{i}$ to $X_{\rho}$. On the other hand, since $u_{i}$ maps to zero in $\operatorname{Pic}\left(X_{\omega} \backslash q_{\omega}^{-1}(Z)\right), C_{i}$ must be a principal divisor, i.e., there exists a rational function $\alpha$ on $X_{\omega}$ with divisor of zeros and poles $(\alpha)=C_{i}+a Z_{\omega}$, where $Z_{\omega}$ is as in the proof of Corollary 5.8 , the single irreducible component of $q_{\omega}^{-1}(Z)$ of codimension one. Thus $C_{i}+a Z_{\omega} \sim 0$ on $X_{\omega}$, and the restriction of $C_{i}+a Z_{\omega}$ to $X_{\rho}$ is zero. But if $T_{e_{1} e_{2}}^{\rho}=0, m_{e_{1} e_{2}}^{\rho}=m_{f \circ h}=0$, so $n_{f \circ h}=0$, and so it follows from Remark 1.56 and Theorem 3.28 that $Z_{\omega}$ is disjoint from $X_{\rho}$. Thus $C_{i}$ restricts to zero on $X_{\rho}$ and $\check{D}\left(u_{i}, k_{i}, f \circ h\right)=0$. Thus $0=\check{D}\left(\lambda_{k_{i}}, k_{i}, f \circ h\right)=\check{D}\left(F_{S, \bar{s}}(h)^{*}\left(\lambda_{k_{i}}\right), g_{i}, f\right)$ as desired.

We then conclude that $\check{D}\left(\lambda_{g_{i}}, g_{i}, f\right)=\check{D}\left(\lambda_{h}, g_{i}, f\right)$. But if $T_{e_{1} e_{2}}^{\rho}=0$, then $\check{D}\left(\lambda_{h}, g_{i}, f\right)$ is also independent of $i$, so $\check{D}\left(\lambda_{g_{1}}, g_{1}, f\right)=\check{D}\left(\lambda_{g_{2}}, g_{2}, f\right)$.

To conclude for the general case, we can use the above special case if there is a sequence of one-dimensional subcells $\omega_{1}, \ldots, \omega_{m}$ of $\tau$ with endpoints of $\omega_{i}$ being $w_{i}$ and $w_{i+1}$, with $v_{1}=w_{1}$ and $v_{2}=w_{m+1}$, such that $T_{w_{i} w_{i+1}}^{\rho}=0$. To see this, consider the fan $\check{\Sigma}_{\rho}$, with the function $\check{\psi}_{\rho}$ of Remark 1.56. By positivity $\check{\psi}_{\rho}$ is convex. However, $\check{\psi}_{\rho}$ is determined by the same element of $\Lambda_{\rho}$ on both the cones $\check{v}_{1}$ and $\check{v}_{2}$ of $\check{\Sigma}_{\rho}$ if $m_{e_{1} e_{2}}^{\rho}$. By convexity of $\check{\psi}_{\rho}$, one can then find a sequence of maximal cones $\check{w}_{1}, \ldots, \check{w}_{m+1}$ of $\check{\Sigma}_{\rho}$ containing $\check{\tau}$ for which $\check{\psi}_{\rho}$ is still given by the same element of $\Lambda_{\rho}$, and such that $\check{w}_{i} \cap \breve{w}_{i+1}$ is a codimension one cone, i.e., $\check{\omega}_{i}$ for some dimension one cell $\omega_{i} \subseteq \tau$. This proves the claim.

Step 4. The inclusion $H^{1}(\mathscr{W}, \mathcal{A f f}(B, \mathbb{Z})) \hookrightarrow H^{1}\left(Q^{\bullet}\right)$ is an isomorphism when tensored with $\mathbb{Q}$, and is an isomorphism over $\mathbb{Z}$ if the additional hypotheses on the $\Delta(\tau)$ 's are satisfied.

Proof of Step 4. Given a 1-cocycle $\left(\lambda_{e}\right)$ representing an element of $H^{1}\left(Q^{\bullet}\right)$, we would like to find $\left(t_{\tau}\right)_{\tau \in \mathscr{P}}$ with $t_{\tau} \in \Gamma\left(X_{\tau} \backslash q_{\tau}^{-1}(Z), \mathcal{M}_{\tau}^{\mathrm{gp}}\right) / k^{\times}$ such that for $e: \tau_{1} \rightarrow \tau_{2}, t_{\tau_{2}}+\lambda_{e}-F_{S, \bar{s}}(e)^{*}\left(t_{\tau_{1}}\right)$ is in the image of $\Gamma\left(W_{e}, \mathcal{A} f f(B, \mathbb{Z})\right)$. This is proved in exactly the same way as the proof of Theorem 5.2, (1), with everything being dual (and additive). Since the proof would be essentially word for word the same, we do not repeat it here, but note the one problem which appears. The analogue of the function $u_{\tau}$ constructed in the proof of Theorem 5.2, (1), is first defined as a function $u_{\tau}:\left(T_{\Delta_{1}} \cap \Lambda_{z}\right)+\cdots+\left(T_{\Delta_{p}} \cap \Lambda_{z}\right) \rightarrow \mathbb{Z}$. However, in general, this extends to a function $\Lambda_{z} \rightarrow \mathbb{Z}$ only after multiplying $u_{\tau}$ by 
an integer. This is the same as multiplying the representative $\left(\lambda_{e}\right)$ by an integer. Thus we only get an isomorphism over $\mathbb{Q}$. If, however, $\Delta(\tau)$ is always a standard simplex, then $\Lambda_{z}$ splits as $\left(T_{\Delta_{1}} \cap \Lambda_{z}\right) \oplus \cdots \oplus\left(T_{\Delta_{p}} \cap\right.$ $\left.\Lambda_{z}\right) \oplus L$ for some free $\mathbb{Z}$-module $L$, and then $u_{\tau}$ can be defined.

Step 5. To complete the proof, we now take the long exact cohomology sequence associated to the exact sequence $0 \rightarrow \Gamma\left(X \backslash Z, \mathcal{I}^{\bullet}\right) \rightarrow$ $\Gamma\left(X \backslash Z, \mathscr{C}^{\bullet}\right) \rightarrow Q^{\bullet} \rightarrow 0$, and use steps 1,2 and 4 , as well as Lemma 5.13. Finally, $H^{p}(\mathscr{W}, \mathcal{A} f f(B, \mathbb{Z}))=H^{p}(B, \mathcal{A} f f(B, \mathbb{Z}))$, as can be shown in the same manner as in Lemma 5.5. Any elementary simplex of dimension $\leq 2$ is standard, hence the last statement follows if $\operatorname{dim} B \leq 3$. q.e.d.

Remark 5.20. In case $B$ is compact every global integral affine function is constant by Proposition 1.40 . Hence $H^{0}(B, \mathcal{A} f f(B, \mathbb{Z}))=\mathbb{Z}$ and $\rho \in H^{0}\left(X \backslash Z, \mathcal{M}_{X}^{\mathrm{gp}}\right)$ maps to a generator. Thus the sequence in the theorem splits into two short exact sequences, the first just giving

$$
H^{0}\left(X \backslash Z, \mathcal{M}_{X}^{\mathrm{gp}}\right)=k^{\times} \times \mathbb{Z}
$$

Taking into account Proposition 2.38 the second sequence reads

$$
\begin{aligned}
0 \longrightarrow \operatorname{Pic}^{\tau}(X) \longrightarrow H^{1}\left(X \backslash Z, \mathcal{M}_{X}^{\mathrm{gp}}\right) & \\
& \longrightarrow \operatorname{ker}\left(H^{1}\left(Q^{\bullet}\right) \rightarrow H^{2}\left(B, k^{\times}\right)\right) \longrightarrow 0 .
\end{aligned}
$$

Thus we see that the logarithmic Picard group $H^{1}\left(X \backslash Z, \mathcal{M}_{X}^{\mathrm{gp}}\right)$ contains $\operatorname{Pic}^{\tau}(X)$ as a subgroup, and the quotient is discrete.

From the exact sequence of Proposition 1.42, we get

Corollary 5.21. If $H^{i}(B, \mathbb{Z})=0$ and $H^{i}\left(B, k^{\times}\right)=0$ for $i=1,2$ and $\Delta(\tau)$ is a standard simplex for each $\tau \in \mathscr{P}$, then

$$
H^{1}\left(X \backslash Z, \mathcal{M}_{X}^{\mathrm{gp}}\right) \cong H^{1}\left(B, i_{*} \check{\Lambda}\right) .
$$

Without these hypotheses, if $H^{i}(B, \mathbb{Q})=0$ for $i=1,2$,

$$
H^{1}\left(X \backslash Z, \mathcal{M}_{X}^{\mathrm{gp}}\right) \otimes \mathbb{Q} \cong H^{1}\left(B, i_{*} \check{\Lambda}\right) \otimes \mathbb{Q} .
$$

\section{Example 5.22.}

1) Suppose $B=\mathbb{R} / n \mathbb{Z}, \mathscr{P}$ arbitrary. Then $\mathcal{A} f f(B, \mathbb{Z})$ is in fact a local system of rank 2 on $B$ with monodromy $\left(\begin{array}{ll}1 & n \\ 0 & 1\end{array}\right)$. Also, $X=$ $X_{0}(B, \mathscr{P}, s)$ is a cycle of rational curves, and it is not difficult to see that $H^{0}\left(X, \mathcal{M}_{X}^{\mathrm{gp}}\right)=k^{\times} \times \mathbb{Z}$, generated by constant functions and $\rho$. Then the exact sequence of Theorem 5.19 gives

$$
0 \rightarrow k^{\times} \rightarrow k^{\times} \times \mathbb{Z} \rightarrow \mathbb{Z} \rightarrow k^{\times} \rightarrow H^{1}\left(X, \mathcal{M}_{X}^{\text {gp }}\right) \rightarrow \mathbb{Z} \rightarrow 0,
$$

so $H^{1}\left(X, \mathcal{M}_{X}^{\mathrm{gp}}\right)=k^{\times} \times \mathbb{Z}$. See [26] and [40] for discussions of $H^{1}\left(X, \mathcal{M}_{X}^{\mathrm{gp}}\right)$ for $X$ a curve.

2) Suppose $B$ is the base of an elliptic fibration on a K3 surface (Example 1.17) which is integral and has 24 singular points, and a 
polyhedral decomposition $\mathscr{P}$ with $B, \mathscr{P}$ simple (or alternatively, construct $B$ as the dual intersection complex of a suitable toric degeneration of $\mathrm{K} 3$ surfaces $)$. Set $X=X_{0}(B, \mathscr{P}, s)$. One can show that $H^{1}\left(B, i_{*} \check{\Lambda}\right)=\mathbb{Z}^{20}$, and $H^{1}(B, \mathcal{A} f f(B, \mathbb{Z}))$ is the kernel of a boundary map $H^{1}\left(B, i_{*} \check{\Lambda}\right) \rightarrow H^{2}(B, \mathbb{Z})=\mathbb{Z}$, and hence is either 19 or 20 dimensional. Finally we have

$$
0 \rightarrow H^{1}\left(X \backslash Z, \mathcal{M}_{X}^{\mathrm{gp}}\right) \rightarrow H^{1}(B, \mathcal{A} f(B, \mathbb{Z})) \rightarrow k^{\times}
$$

from Theorem 5.19. The last map will depend on the choice of open gluing data, giving a range of possible values for $H^{1}\left(X \backslash Z, \mathcal{M}_{X}^{\text {gp }}\right)$.

\subsection{Mirror symmetry and conclusions. We first define}

Definition 5.23. Let $X$ be a toric log Calabi-Yau space over an algebraically closed field $k$, with dual intersection complex $B$. Then the log Kähler moduli space of $X$ is defined to be $H^{1}\left(B, i_{*} \check{\Lambda} \otimes \mathbb{G}_{m}(k)\right)$.

Theorem 5.24. Let $(X, \mathcal{L})$ be a polarized log Calabi-Yau space with $(B, \mathscr{P}, \varphi)$ being its degeneration data. Suppose $B$ is positive and simple. If $(\check{B}, \check{P}, \check{\varphi})$ is the discrete Legendre transform of $(B, \mathscr{P}, \varphi)$, then the set of positive log Calabi-Yau spaces with dual intersection complex $(\check{B}, \check{\mathscr{P}})$ modulo isomorphism preserving $B$ is naturally isomorphic to the log Kähler moduli space of $X$.

Proof. This follows from Theorem 5.4 and the fact that $\check{\Lambda}^{B_{0}} \cong \Lambda^{\check{B}_{0}}$ by Proposition 1.50 .

q.e.d.

Of course, at this point the reader may object that we have simply defined the log Kähler moduli space to make the above theorem work. Thus a bit more justification seems to be in order. The calculations of $\S 5.2$ say that $H^{1}\left(B, i_{*} \check{\Lambda}\right)$ has something to do with the logarithmic Picard group, which in turn should have something to do with the smoothing of $X$, should such exist. This is perhaps weaker evidence than one would like. However, in [21], we will define logarithmic Hodge groups $H^{p, q}(X)$ for $X$ a log Calabi-Yau space, and compute these groups. In certain cases, these coincide with $H^{q}\left(B, i_{*} \bigwedge^{p} \check{\Lambda} \otimes_{\mathbb{Z}} k\right)$. Furthermore, we will show in these cases these groups have the same dimension as the dimension of the Hodge groups of a smoothing. Thus $H^{1,1}(X)$ is directly related to the Kähler moduli space. In addition, $H^{n-1,1}(X)=H^{1}\left(B, i_{*} \bigwedge^{n-1} \check{\Lambda} \otimes_{\mathbb{Z}} k\right)=H^{1}\left(B, i_{*} \Lambda \otimes_{\mathbb{Z}} k\right)$ if the holonomy of $B$ is contained in $\mathbb{Z}^{n} \rtimes \mathrm{SL}_{n}(\mathbb{Z})$ rather than in $\mathbb{Z}^{n} \rtimes \mathrm{GL}_{n}(\mathbb{Z})$. As $H^{n-1,1}(X)$ is then the same dimension as the moduli space of a smoothing of $X$, we see that the moduli space of log Calabi-Yau spaces $H^{1}\left(B, i_{*} \Lambda \otimes \mathbb{G}_{m}(k)\right)$ has the correct dimension.

Another motivation comes from [16]. There it was suggested that if $f: X \rightarrow B$ was a simple torus fibration, it was natural to consider the $B$-field living in the group $H^{1}\left(B, R^{1} f_{*}(\mathbb{R} / \mathbb{Z})\right)$. This was a somewhat 
different proposal than is usually considered in the physics literature, in that it depends not just on $X$ but on the fibration. However, we feel it is the natural group, and some moral justification for this was given in [16]. (The reader should of course keep in mind we do not yet have a satisfactory mathematical definition of mirror symmetry, and therefore it does not make sense to prove such assertions).

Thus this suggests that the complexified Kähler moduli space lies naturally in

$$
H^{1}\left(B, R^{1} f_{*}(\mathbb{C} / \mathbb{Z})\right)=H^{1}\left(B,\left(R^{1} f_{*} \mathbb{Z}\right) \otimes \mathbb{C}^{\times}\right) .
$$

On the other hand, if $f$ was a special Lagrangian fibration, the base $B$ would carry two affine structures (with singularities) related by a Legendre transform $[\mathbf{2 4}]$. In the one coming from the complex structure, we naturally have $R^{1} f_{*} \mathbb{Z} \cong i_{*} \check{\Lambda}$. Thus in this context, the definition of the log Kähler moduli space as $H^{1}\left(B, i_{*} \check{\Lambda} \otimes \mathbb{G}_{m}(k)\right)$ appears to be the correct one. The fact that this is indeed isomorphic to the moduli of log Calabi-Yau spaces for a "mirror" supports this.

At any rate, we have now achieved mirror symmetry for polarized log Calabi-Yau spaces:

Definition 5.25. Let $(X, \mathcal{L})$ and $(\check{X}, \check{\mathcal{L}})$ be two polarized log CalabiYau spaces with degeneration data $(B, \mathscr{P}, \varphi)$ and $(\check{B}, \check{\mathscr{P}}, \check{\varphi})$ respectively. If these are related by a discrete Legendre transform, we say $(X, \mathcal{L})$ and $(\check{X}, \check{\mathcal{L}})$ are a mirror pair of log Calabi-Yau spaces. In particular, if $B$ (and hence $\check{B}$ ) are positive and simple, then there is a canonical isomorphism between the log Kähler moduli space of $X$ and the set of log Calabi-Yau spaces with dual intersection complex $(B, \mathscr{P})$ modulo isomorphism preserving $B$.

Example 5.26. Let $\Xi$ be a reflexive polytope, and consider a toric degeneration $\mathcal{X} \rightarrow \mathcal{S}$ constructed as in Example 4.29, and let $\check{\mathcal{X}} \rightarrow \mathcal{S}$ be constructed similarly using the dual polytope $\Xi^{*}$. Let $X=\mathcal{X}_{0}^{\dagger}$ and $\check{X}=\check{\mathcal{X}}_{0}^{\dagger}$. Polarize $X$ and $\check{X}$ by the anti-canonical divisors $\mathcal{L}=$ $\left.\mathcal{O}_{\mathbb{P}_{\Xi^{*}}}(1)\right|_{X}$ and $\check{\mathcal{L}}=\left.\mathcal{O}_{\mathbb{P}_{\Xi}}(1)\right|_{\check{X}}$ respectively. Then by Example 1.53, it follows that $(X, \mathcal{L})$ and $(\check{X}, \check{L})$ are a mirror pair. Note in this case we don't expect $(B, \mathscr{P})$ to be simple, so we don't expect the isomorphisms between Kähler and complex moduli. This is because the generic fibres $\mathcal{X}_{\eta}$ and $\check{\mathcal{X}}_{\eta}$ are not in general smooth, or MPCP resolutions. Different polarizations and degenerations are required to achieve this, see [18].

Our goal, of course, is not just a version of mirror symmetry for log Calabi-Yau spaces. We still need to address a number of questions to make use of the program we have begun here. Principal among these are:

1) We are really interested in toric degenerations of Calabi-Yau varieties $f: \mathcal{X} \rightarrow \mathcal{S}$. To use the mirror symmetry above to study 
mirror symmetry of non-singular Calabi-Yau varieties, we need to compare the log invariants of $X=\mathcal{X}_{0}^{\dagger}$ with the usual invariants of the generic fibre $\mathcal{X}_{\bar{\eta}}$. For example, we expect in the simple case that $h^{1, n-1}\left(\mathcal{X}_{\bar{\eta}}\right)$ coincides with the dimension of the moduli of $\log$ Calabi-Yau spaces with the same dual intersection complex as $X$. This should follow from the right sort of base-change results. Many of the necessary ideas for proving such results are already present in the literature for log smooth morphisms; we however have the singular set $Z$ to worry about, which complicates things.

2) To turn these ideas into a genuine mirror symmetry construction, we need to study the log deformation theory of log Calabi-Yau spaces, à la Kato [27] and Kawamata-Namikawa [29]. Again, we hope in the simple case that a log Calabi-Yau space is always the central fibre of a toric degeneration. The Bogomolov-TianTodorov type results of [29] give some moral support that such should be true. However, the presence of the singular set means we cannot apply known results off the shelf.

3) We believe this approach will provide a very general mirror symmetry construction. It incorporates Batyrev-Borisov duality, as is explained in [18]. It also includes mirror symmetry for abelian varieties, but it would be nice to know it gives some genuinely new constructions.

4) This approach should be connected more explicitly with the SYZ approach. It is clear that our approach is a discretization of the SYZ approach, in that the Legendre transform is replaced by the discrete Legendre transform. However, we would like to see torus fibrations when we work over $\mathbb{C}$. Some results in this direction were stated in [19]. The proof of these results will appear in [20], along with more precise results in the simple case.

5) Much more generally, the log philosophy says that things you want to compute on smoothings can be computed using logarithmic analogues on the degenerations. For example, we hope it should be possible to define log Gromov-Witten invariants [45] which will coincide with those of a smoothing. The results of [34] and [36] suggest that this should be possible by counting certain piecewise straight graphs on $B$ ("tropical curves"). In addition, the philosophy presented in this paper is that invariants of toric log Calabi-Yau spaces can be computed doing calculations on $B$. This is the general philosophy we hope to push further in order to understand mirror symmetry.

The sequel to this paper [21] will address questions (1) and (2). However, our work in this direction is still too preliminary to give specific statements here. 
So there is still much work to be done to make the logarithmic approach to mirror symmetry realise its full potential. We hope the results presented here already provide ample evidence that logarithmic degeneration data capture the essential features of mirror symmetry.

\section{Appendix A. Simplicial and Polyhedral Complexes}

The purpose of this appendix is to collect some facts on complexes arising from a polyhedral decomposition. We first treat the case of abelian groups and then sheafify.

A.1. Barycentric complexes. Let $\mathscr{P}$ be the face poset of a $d$-dimensional polytope $\Xi$. Assume that $\left(M_{\tau}\right)_{\tau \in \mathscr{P}}$ is a category of abelian groups indexed by $\mathscr{P}$, that is, a functor $\underline{\operatorname{Cat}}(\mathscr{P}) \rightarrow \underline{\mathrm{Ab}}$. For $\tau, \sigma \in \mathscr{P}$, $\tau \subset \sigma$ denote by $\varphi_{\sigma \tau}: M_{\tau} \rightarrow M_{\sigma}$ the homomorphism of abelian groups thus given. Then $\varphi_{\tau \tau}=$ id and $\varphi_{\sigma \tau} \circ \varphi_{\tau \omega}=\varphi_{\sigma \omega}$ whenever $\omega \subset \tau \subset \sigma$. For notational convenience we write $\varphi_{\sigma}(f)$ instead of $\varphi_{\sigma \tau}(f)$ whenever $f \in M_{\tau}$ and $\tau \subset \sigma$. Denote by $\mathscr{P}{ }^{[k]} \subset \mathscr{P}$ the subset of $k$-dimensional faces.

The barycentric cochain complex $\left(C_{\mathrm{bct}}^{\bullet}, d_{\mathrm{bct}}^{\bullet}\right)$ associated to $\left(M_{\sigma}\right)$ is the complex of abelian groups $C_{\mathrm{bct}}^{k}=\bigoplus_{\sigma_{0} \subsetneq \sigma_{1} \subsetneq \ldots \subsetneq \sigma_{k}} M_{\sigma_{k}}$ of simplicial cochains with differentials

$$
\left(d_{\mathrm{bct}}^{k}\left(f_{\sigma_{0} \sigma_{1} \ldots \sigma_{k}}\right)\right)_{\sigma_{0} \sigma_{1} \ldots \sigma_{k+1}}=\sum_{i=0}^{k+1}(-1)^{i} \varphi_{\sigma_{k+1}}\left(f_{\sigma_{0} \ldots \widehat{\sigma}_{i} \ldots \sigma_{k+1}}\right) .
$$

Here and in the following entries with a hat are to be omitted. This is indeed a complex as one easily checks. There is generally no reason for this complex to be acyclic, but it will be once $\left(M_{\sigma}\right)$ has the following extension property. For $\mathscr{P}^{\prime} \subset \mathscr{P}$ let us call a collection $f_{\tau} \in M_{\tau}$, $\tau \in \mathscr{P}^{\prime}$, compatible if $\varphi_{\sigma}\left(f_{\tau}\right)=\varphi_{\sigma}\left(f_{\tau^{\prime}}\right)$ for any $\tau, \tau^{\prime} \in \mathscr{P}^{\prime}, \sigma \in \mathscr{P}$, $\tau, \tau^{\prime} \subset \sigma$. We consider the following condition.

Any compatible collection $\left(f_{\sigma}\right)_{\sigma \in \mathscr{P} \prime}$ indexed by any $\mathscr{P}^{\prime} \subset \mathscr{P}$

(*) extends to a compatible collection $\left(g_{\sigma}\right)_{\sigma \in \mathscr{P}}$, that is, $g_{\sigma} \in M_{\sigma}$ and $g_{\sigma}=f_{\sigma}$ for $\sigma \in \mathscr{P}^{\prime}$.

Proposition A.1. If Condition (*) holds then the barycentric cochain complex associated to $\left(M_{\sigma}\right)$ is acyclic.

Proof. We wish to write a simplicial cocycle $\left(f_{\sigma_{0} \ldots \sigma_{k}}\right)_{\left(\sigma_{0}, \ldots, \sigma_{k}\right)}$ as the coboundary of a simplicial $(k-1)$-cochain $\left(g_{\sigma_{0} \ldots \sigma_{k-1}}\right)_{\left(\sigma_{0}, \ldots, \sigma_{k-1}\right)}$. We construct $g_{\sigma_{0} \cdots \sigma_{k-1}}$ by descending induction on $m=\operatorname{dim} \sigma_{k-1}=d+$ $1, \ldots, 0$. The induction hypothesis is that

$$
f_{\sigma_{0} \ldots \sigma_{k}}=\sum_{i=0}^{k}(-1)^{i} \varphi_{\sigma_{k}}\left(g_{\sigma_{0} \ldots \widehat{\sigma}_{i} \ldots \sigma_{k}}\right),
$$


whenever $\operatorname{dim} \sigma_{k-1} \geq m$. The induction starts at $m=d+1$. Condition (A.1) is empty at this stage because $\mathscr{P}$ is the face poset of a $d$ dimensional polytope. For the induction step consider $\sigma_{0} \subsetneq \ldots \subsetneq \sigma_{k-1}$ with $\operatorname{dim} \sigma_{k-1}=m-1$. We want to find $g_{\sigma_{0} \ldots \sigma_{k-1}}$ such that for any $\sigma_{k}$ containing $\sigma_{k-1}$

$$
(-1)^{k} \varphi_{\sigma_{k}}\left(g_{\sigma_{0} \ldots \sigma_{k-1}}\right)=f_{\sigma_{0} \ldots \sigma_{k}}-\sum_{i=0}^{k-1}(-1)^{i} g_{\sigma_{0} \ldots \widehat{\sigma}_{i} \ldots \sigma_{k}} .
$$

This is the required equation for $f_{\sigma_{0} \ldots \sigma_{k}}$, and all terms on the right-hand side are known inductively. Now view the right-hand side as a collection of elements indexed by $\mathscr{P}^{\prime}=\left\{\sigma_{k} \mid \sigma_{k-1} \subset \sigma_{k}\right\}$. By Condition (*) $g_{\sigma_{0} \ldots \sigma_{k-1}}$ then exists if this collection is compatible, that is, if for any $\sigma_{k+1} \supset \sigma_{k-1}$ the expression

$$
\varphi_{\sigma_{k+1}}\left(f_{\sigma_{0} \ldots \sigma_{k}}-\sum_{i=0}^{k-1}(-1)^{i} g_{\sigma_{0} \ldots \widehat{\sigma}_{i} \ldots \sigma_{k}}\right)
$$

does not depend on $\sigma_{k}$ for $\sigma_{k-1} \subset \sigma_{k} \subset \sigma_{k+1}$. For $i \leq k$ the induction hypothesis implies

$$
\begin{aligned}
f_{\sigma_{0} \ldots \widehat{\sigma}_{i} \ldots \sigma_{k+1}}= & \sum_{j=0}^{i-1}(-1)^{j} g_{\sigma_{0} \ldots \widehat{\sigma}_{j} \ldots \widehat{\sigma}_{i} \ldots \sigma_{k+1}} \\
& -\sum_{j=i+1}^{k+1}(-1)^{j} \varphi_{\sigma_{k+1}}\left(g_{\sigma_{0} \ldots \widehat{\sigma}_{i} \ldots \widehat{\sigma}_{j} \ldots \sigma_{k+1}}\right) .
\end{aligned}
$$

Plugging this into the cocycle condition

$$
\varphi_{\sigma_{k+1}}\left(f_{\sigma_{0} \ldots \sigma_{k}}\right)=(-1)^{k} \sum_{i=0}^{k}(-1)^{i} f_{\sigma_{0} \ldots \widehat{\sigma}_{i} \ldots \sigma_{k+1}},
$$

the first term of (A.2) gives $f_{\sigma_{0} \ldots \sigma_{k-1} \sigma_{k+1}}(i=k)$ plus a sum over $g_{\sigma_{0} \ldots \widehat{\sigma}_{i} \ldots \widehat{\sigma}_{j} \ldots \sigma_{k+1}}$. For $0 \leq i<j<k$ the coefficient of $g_{\sigma_{0} \ldots \widehat{\sigma}_{i} \ldots \widehat{\sigma}_{j} \ldots \sigma_{k+1}}$ is $(-1)^{k}$ times $(-1)^{i}\left(-(-1)^{j}\right)+(-1)^{j}(-1)^{i}=0$. Contributions involving $\varphi_{\sigma_{k+1}}\left(g_{\sigma_{0} \ldots \widehat{\sigma}_{i} \ldots \sigma_{k}}\right)$ come from the second term in (A.2) and from $j=k+1$; they cancel as well. Thus (A.2) equals

$$
f_{\sigma_{0} \ldots \sigma_{k-1} \sigma_{k+1}}+(-1)^{k} \sum_{i=0}^{k-1}(-1)^{i}(-1)^{k}\left(-g_{\sigma_{0} \ldots \widehat{\sigma}_{i} \ldots \widehat{\sigma}_{k} \sigma_{k+1}}\right) .
$$

This shows the claimed independence of (A.2), and hence the existence of $g_{\sigma_{0} \ldots \sigma_{k-1}}$. 
A.2. Polyhedral complexes. The second type of complex that we deal with is non-simplicial. We start with the same kind of system $\left(M_{\sigma}\right)_{\sigma \in \mathscr{P}}$ of abelian groups as in the previous subsection. The following construction depends on a choice of orientations of the faces of $\Xi$. Comparison with the standard orientation of the boundary of a face gives a $\operatorname{sign} \operatorname{sgn}(\tau, \omega)$ whenever $\tau \subset \omega$ and $\operatorname{dim}(\omega)=\operatorname{dim}(\tau)+1$. Similarly, if $\sigma_{0} \subset \ldots \subset \sigma_{k}$ and $\operatorname{dim} \sigma_{i}=i$ comparison of the natural orientation of the simplex $\left(\sigma_{0}, \ldots, \sigma_{k}\right)$ of the barycentric subdivision of $\sigma_{k}$ with the chosen orientation defines a sign $\operatorname{sgn}\left(\left(\sigma_{0}, \ldots, \sigma_{k}\right), \sigma_{k}\right)$.

We can then define the polyhedral cochain complex $\left(C_{\mathrm{phd}}^{\bullet}, d_{\mathrm{phd}}^{\bullet}\right)$ associated to $\left(M_{\sigma}\right)$ as the complex of abelian groups $C_{\mathrm{phd}}^{k}=\bigoplus_{\tau \in \mathscr{P}[k]} M_{\tau}$ of polyhedral cochains with differentials

$$
d_{\mathrm{phd}}^{k}:\left(f_{\tau}\right)_{\tau \in \mathscr{P}[k]} \longmapsto\left(\sum_{\tau \subset \omega, \tau \in \mathscr{P}[k]} \operatorname{sgn}(\tau, \omega) \varphi_{\omega \tau}\left(f_{\tau}\right)\right)_{\omega \in \mathscr{P}[k+1]} .
$$

To check that the composition of differentials vanishes, recall that if $\omega$ is a $(k+1)$-dimensional polytope and $\sigma \subset \omega$ has codimension 2 then there are exactly two $k$-dimensional facets $\tau^{ \pm} \subset \omega$ with $\sigma \subset \tau^{ \pm} \subset \omega$. Because the orientations on $\sigma$ induced by the orientations of $\tau^{+}, \tau^{-}$as facets of $\omega$ differ, it holds

$$
\operatorname{sgn}\left(\sigma, \tau^{+}\right) \operatorname{sgn}\left(\tau^{+}, \omega\right)=-\operatorname{sgn}\left(\sigma, \tau^{-}\right) \operatorname{sgn}\left(\tau^{-}, \omega\right) .
$$

Therefore,

$$
\begin{aligned}
& \left(d_{\mathrm{phd}}^{k} \circ d_{\mathrm{phd}}^{k-1}\left(f_{\sigma}\right)\right)_{\omega}=\sum_{\sigma \subset \tau \subset \omega} \operatorname{sgn}(\sigma, \tau) \operatorname{sgn}(\tau, \omega)\left(\varphi_{\omega \tau} \circ \varphi_{\tau \sigma}\right)\left(f_{\sigma}\right) \\
& \quad=\sum_{\substack{\sigma \subset \omega \\
\operatorname{dim} \sigma=k-1}}\left(\operatorname{sgn}\left(\sigma, \tau^{+}\right) \operatorname{sgn}\left(\tau^{+}, \omega\right)+\operatorname{sgn}\left(\sigma, \tau^{-}\right) \operatorname{sgn}\left(\tau^{-}, \omega\right)\right) \varphi_{\omega \sigma}\left(f_{\sigma}\right) \\
& \quad=0 .
\end{aligned}
$$

Proposition A.2. If Condition (*) holds then the polyhedral cochain complex associated to $\left(M_{\sigma}\right)$ is acyclic.

Proof. Let $\left(f_{\tau}\right)_{\tau \in \mathscr{P}[k]} \in C_{\text {phd }}^{k}$ fulfill the cocycle condition that for any $\omega \in \mathscr{P}[k+1]$

$$
\sum_{\tau \subset \omega} \operatorname{sgn}(\tau, \omega) \cdot \varphi_{\omega \tau}\left(f_{\tau}\right)=0 .
$$

For any $(k-1)$-cell $\sigma$ we have to find $g_{\sigma} \in M_{\sigma}$ with the property that for any $\tau \in \mathscr{P}^{[k]}$

$$
f_{\tau}=\sum_{\sigma \subset \tau} \operatorname{sgn}(\sigma, \tau) \cdot \varphi_{\tau \sigma}\left(g_{\sigma}\right)
$$

The construction of $g_{\sigma}$ is in three steps. Steps 1 and 2 produce a simplicial cocycle out of $f_{\tau}$, which by Proposition A.1 may then be written 
as a simplicial coboundary; Step 3 translates back to the polyhedral setting.

Step 1. The aim of the first two steps is to transform, in a sense, $\left(f_{\tau}\right)$ to a cocyle in $C_{\mathrm{bct}}^{k}$. For each $\tau \in \mathscr{P}^{[k]}$ choose a maximal chain $\tau_{0} \subsetneq$ $\tau_{1} \subsetneq \ldots \subsetneq \tau_{k}=\tau$ and write $\underline{\tau}=\left(\tau_{0}, \ldots, \tau_{k}\right)$. For $\sigma_{0} \subset \sigma_{1} \subset \ldots \subset \sigma_{k}$ with $\operatorname{dim} \sigma_{i}=i$ we then put

$$
f_{\sigma_{0} \ldots \sigma_{k}}= \begin{cases}(-1)^{k} \operatorname{sgn}(\underline{\tau}, \tau) f_{\tau} & ,\left(\sigma_{0}, \ldots, \sigma_{k}\right)=\left(\tau_{0}, \ldots, \tau_{k}\right) \\ 0 & , \text { otherwise. }\end{cases}
$$

It remains to define $f_{\sigma_{0} \ldots \sigma_{k-1} \omega}$ for $\operatorname{dim} \omega>k$.

In this step we deal with the case $\operatorname{dim} \omega=k+1$. Since $\omega$ is a polytope it can be built up by consecutively attaching $(k+1)$-simplices from its barycentric subdivision, keeping it a cell in each step. This means that there is an order among the $(k+1)$-simplices $\left(\sigma_{0}, \ldots, \sigma_{k}, \sigma_{k+1}=\omega\right)$ with the property that any two consecutive elements intersect along a $k$-simplex, and all but the last cell are attached along a union of at most $k$ of the $k+1$ facets not lying in $\partial \omega$. Let $\Xi_{\kappa}, \kappa=0, \ldots, K$ be the cell obtained after the $\kappa$-th attachment. Assume $f_{\sigma_{0} \ldots \sigma_{k}}$ is constructed for all $k$-simplices contained in $\Xi_{\kappa}$ for $0 \leq \kappa \leq \kappa_{0}<K$, and $\Xi_{\kappa_{0}}$ equals $\Xi_{\kappa_{0}-1}$ with the $(k+1)$-simplex $\left(\sigma_{0}, \ldots, \sigma_{k+1}=\omega\right)$ attached. Then $f_{\sigma_{0} \ldots \widehat{\sigma}_{i} \ldots \sigma_{k+1}}$ is not defined for a non-empty subset $I \subset\{0, \ldots, k\}$. Put $f_{\sigma_{0} \ldots \widehat{\sigma}_{i} \ldots \sigma_{k+1}}=0$ for $i<\max (I), i \in I$, and

$$
f_{\sigma_{0} \ldots \widehat{\sigma}_{\max (I)} \ldots \sigma_{k+1}}=(-1)^{\max (I)+1} \sum_{i \in\{0, \ldots, \max (I), \ldots, k+1\}}(-1)^{i} \varphi_{\sigma_{k+1}}\left(f_{\sigma_{0} \ldots \widehat{\sigma}_{i} \ldots \sigma_{k+1}}\right),
$$

to fulfill the cocycle condition on $\left(\sigma_{0}, \ldots, \sigma_{k+1}\right)$. By induction from $\kappa_{0}=$ 0 to $\kappa_{0}=K-1$ this determines $f_{\sigma_{0} \ldots \sigma_{k}}$ satisfying the cocycle condition on every $(k+1)$-simplex of the barycentric subdivision of $\omega$ except possibly on the one $\left(\sigma_{0}, \ldots, \sigma_{k+1}\right)$ attached in the $K$-th step. Adding the already known cocycle conditions on all other $(k+1)$-simplices contained in $\omega$ gives the expression

$$
\sum_{\sigma_{0} \subsetneq \ldots \subsetneq \sigma_{k+1}} \operatorname{sgn}\left(\left(\sigma_{0}, \ldots, \sigma_{k+1}\right), \omega\right) \sum_{i=0}^{k+1}(-1)^{i} \varphi_{\omega}\left(f_{\sigma_{0} \ldots \widehat{\sigma}_{i} \ldots \sigma_{k+1}}\right) .
$$

For $i \leq k$ the coefficient of $\varphi_{\omega}\left(f_{\sigma_{0} \ldots \hat{\sigma}_{i} \ldots \sigma_{k+1}}\right)$ is zero, because this term occurs in the sum exactly twice with opposite signs, cf. (A.3). The remaining expression is

$$
\begin{aligned}
\sum_{\sigma_{0} \subsetneq \ldots \subsetneq \sigma_{k+1}} \operatorname{sgn}\left(\left(\sigma_{0}, \ldots, \sigma_{k+1}\right), \omega\right)(-1)^{k+1} \varphi_{\omega}\left(f_{\sigma_{0} \ldots \sigma_{k}}\right) & \\
= & -\sum_{\tau \in \mathscr{P}[k], \tau \subset \omega} \operatorname{sgn}((\underline{\tau}, \omega), \omega) \operatorname{sgn}(\underline{\tau}, \tau) \varphi_{\omega}\left(f_{\tau}\right) .
\end{aligned}
$$


As $\operatorname{sgn}((\underline{\tau}, \omega), \omega) \operatorname{sgn}(\underline{\tau}, \tau)=\operatorname{sgn}(\tau, \omega)$ this is nothing but $d_{\text {phd }}\left(f_{\tau}\right)_{\tau}$, which vanishes by assumption.

Step 2. Now let us assume $f_{\sigma_{0} \ldots \sigma_{k}}$ has been constructed inductively for $\operatorname{dim} \sigma_{k} \leq m$ fulfilling the cocycle condition on any $m$-simplex. Let $\omega$ be of dimension $m+1>k+1$. Then for $\sigma_{0} \subsetneq \ldots \subsetneq \sigma_{k+1} \subset \partial \omega$ the cocycle condition on $\left(\sigma_{0}, \ldots, \sigma_{k+1}\right)$ holds by induction assumption. Applying $\varphi_{\omega}$ gives $\sum_{i=0}^{k+1}(-1)^{i} \varphi_{\omega}\left(f_{\sigma_{0} \ldots \hat{\sigma}_{i} \ldots \sigma_{k+1}}\right)=0$. Thus $\left(\varphi_{\omega}\left(f_{\sigma_{0} \ldots \hat{\sigma}_{i} \ldots \sigma_{k+1}}\right)\right)_{\sigma_{k+1} \subset \omega}$ is a simplicial $k$-cocycle on $\partial \omega \cong S^{m}$ with values in $M_{\omega}$. But $m>$ $k$ and hence $H^{k}\left(\partial \omega, M_{\omega}\right)=0$ by the universal coefficient theorem. Therefore there exists $\left(h_{\sigma_{0} \ldots \sigma_{k-1}}\right)_{\sigma_{k-1} \subset \partial \omega}$ with $(-1)^{k+1} \varphi_{\omega}\left(f_{\sigma_{0} \ldots \sigma_{k}}\right)=$ $-\sum_{i=0}^{k}(-1)^{i} h_{\sigma_{0} \ldots \hat{\sigma}_{i} \ldots \sigma_{k}}$. Putting $f_{\sigma_{0} \ldots \sigma_{k-1} \omega}:=h_{\sigma_{0} \ldots \sigma_{k-1}}$ the cocycle condition continues to hold on any $\left(\sigma_{0}, \ldots, \sigma_{k+1}\right)$ with $\sigma_{k+1} \subset \omega$.

Step 3. By Proposition A.1 there exists a simplicial $(k-1)$-cocycle $g_{\sigma_{0} \ldots \sigma_{k-1}}$ with $f_{\sigma_{0} \ldots \sigma_{k}}=\sum_{i=0}^{k}(-1)^{i} g_{\sigma_{0} \ldots \hat{\sigma}_{i} \ldots \sigma_{k}}$. Now we want to revert to the polyhedral setting. For $\sigma \in \mathscr{P}^{[k-1]}$ put

$$
g_{\sigma}=\sum_{\sigma_{0} \subsetneq \ldots \subsetneq \sigma_{k-1}=\sigma} \operatorname{sgn}\left(\left(\sigma_{0}, \ldots, \sigma_{k-1}\right), \sigma\right) g_{\sigma_{0} \ldots \sigma_{k-1}} .
$$

Then for any $\tau \in \mathscr{P}^{[k]}$

$$
\begin{aligned}
& \sum_{\sigma \subset \tau, \sigma \in \mathscr{P}[k-1]} \operatorname{sgn}(\sigma, \tau) \varphi_{\tau}\left(g_{\sigma}\right) \\
= & \sum_{\sigma_{0} \subsetneq \ldots \subsetneq \sigma_{k}=\tau} \operatorname{sgn}\left(\left(\sigma_{0}, \ldots, \sigma_{k-1}\right), \sigma_{k-1}\right) \operatorname{sgn}\left(\sigma_{k-1}, \tau\right) \varphi_{\sigma_{k}}\left(g_{\sigma_{0} \ldots \sigma_{k-1}}\right) \\
= & \sum_{\sigma_{0} \subsetneq \ldots \subsetneq \sigma_{k}=\tau} \operatorname{sgn}\left(\left(\sigma_{0}, \ldots, \sigma_{k}\right), \tau\right)(-1)^{k} \sum_{i=0}^{k}(-1)^{i} \varphi_{\sigma_{k}}\left(g_{\sigma_{0} \ldots \widehat{\sigma}_{i} \ldots \sigma_{k}}\right) \\
= & \sum_{\sigma_{0} \subsetneq \ldots \subsetneq \sigma_{k}=\tau} \operatorname{sgn}\left(\left(\sigma_{0}, \ldots, \sigma_{k}\right), \tau\right)(-1)^{k} f_{\sigma_{0} \ldots \sigma_{k}} \\
= & \operatorname{sgn}(\underline{\tau}, \tau)(-1)^{k} f_{\tau_{0} \ldots \tau_{k}}=f_{\tau} .
\end{aligned}
$$

To verify the second equality consider the coefficient of $g_{\sigma_{0} \ldots \widehat{\sigma}_{i} \ldots \sigma_{k}}$. For $0 \leq i \leq k-1$ there are exactly two $i$-cells $\sigma_{i}^{ \pm}$between $\sigma_{i-1}$ (empty for $i=0)$ and $\sigma_{i+1}$. Now $g_{\sigma_{0} \ldots \sigma_{i}^{ \pm} \ldots \sigma_{k}}$ contribute with opposite signs, just as in (A.3). This exhibits $\left(f_{\tau}^{i}\right)_{\tau}$ as polyhedral coboundary as desired. q.e.d.

A.3. Complexes of Sheaves. Let $B$ be an integral affine manifold with singularities, and let $\mathscr{P}$ be a toric polyhedral decomposition on $B$. For every $\sigma \in \mathscr{P}$ put $\mathscr{P}_{\sigma}=\coprod_{\tau \in \mathscr{P}} \operatorname{Hom}(\tau, \sigma)$, partially ordered by $(e: \tau \rightarrow \sigma) \leq\left(e^{\prime}: \tau^{\prime} \rightarrow \sigma\right)$ if there exists $f: \tau \rightarrow \tau^{\prime}$ with $e=e^{\prime} \circ f$. There is a natural order preserving map from $\mathscr{P}_{\sigma}$ to the face poset of $\tilde{\sigma}$. 
If we orient every $\sigma \in \mathscr{P}$, then as in the previous subsection comparison of orientations gives signs $\operatorname{sgn}\left(\sigma_{k-1}, \sigma_{k}\right)$ and $\operatorname{sgn}\left(\left(\sigma_{0} \rightarrow \cdots \rightarrow \sigma_{k}\right), \sigma_{k}\right)$ if $\operatorname{dim} \sigma_{i}=i$.

Now let $s$ be open gluing data for $(B, \mathscr{P})$ over $S$, and let $X=$ $X_{0}(B, \mathscr{P}, s)$. Suppose that for each $\sigma \in \mathscr{P}$, we are given a sheaf $\mathcal{F}_{\sigma}$ on $X_{\sigma}$, (here we write $X_{\sigma}$ for $X_{\sigma} \times S$ ) and for each $e \in \operatorname{Hom}(\tau, \sigma)$ a map

$$
\varphi_{e}: F_{S, \bar{s}}(e)^{-1}\left(\mathcal{F}_{\tau}\right) \rightarrow \mathcal{F}_{\sigma},
$$

compatible with compositions. Analogous to the constructions for abelian groups define the barycentric and polyhedral cochain complexes associated to $\left(\mathcal{F}_{\sigma}\right)_{\sigma \in \mathscr{P}}$ by

$$
\mathscr{C}_{\text {bct }}^{k}=\bigoplus_{\sigma_{0} \rightarrow \cdots \rightarrow \sigma_{k}} q_{\sigma_{k} *} \mathcal{F}_{\sigma_{k}}
$$

and

$$
\mathscr{C}_{\mathrm{phd}}^{k}=\bigoplus_{\sigma \in \mathscr{P}[k]} q_{\sigma *} \mathcal{F}_{\sigma}
$$

respectively, with differentials

$$
\begin{aligned}
& d_{\mathrm{bct}}^{k}\left(f_{\sigma_{0} \rightarrow \cdots \rightarrow \sigma_{k}}\right)=\left(\begin{array}{l}
\sum_{i=0}^{k}(-1)^{i} f_{\sigma_{0} \rightarrow \cdots \rightarrow \widehat{\sigma}_{i} \rightarrow \cdots \sigma_{k+1}} \\
+(-1)^{k+1} \varphi_{\sigma_{k} \rightarrow \sigma_{k+1}}\left(f_{\sigma_{0} \rightarrow \cdots \rightarrow \sigma_{k}}\right)
\end{array}\right)_{\sigma_{0} \rightarrow \cdots \rightarrow \sigma_{k+1}}, \\
& d_{\mathrm{phd}}^{k}\left(f_{\sigma}\right)_{\sigma \in \mathscr{P}[k]}=\left(\sum_{\tau \rightarrow \omega} \operatorname{sgn}(\tau, \omega) \varphi_{\tau \rightarrow \omega}\left(f_{\tau}\right)\right)_{\omega \in \mathscr{P}[k+1]} .
\end{aligned}
$$

We will use the previous two subsections to give a criterion for exactness of $\mathscr{C}_{\text {bct }}^{\bullet}$ and $\mathscr{C}_{\text {phd }}^{\bullet}$. To check exactness, it is enough to do so on stalks. If $\bar{x} \rightarrow X$ is a geometric point, let $\sigma$ be the largest $\sigma \in \mathscr{P}$ such that $\bar{x}$ is in the image under $q_{\sigma}$ of a geometric point of $X_{\sigma}$. Then $q_{\tau *}\left(\mathcal{F}_{\tau}\right)_{\bar{x}}=0$ unless $\tau \subseteq \sigma$. Furthermore, the set of geometric points of $X_{\tau}$ mapping to $\bar{x}$ is in one-to-one correspondence with the set $\operatorname{Hom}(\tau, \sigma)$. If $\bar{y} \rightarrow X_{\tau}$ with $q_{\tau}(\bar{y})=\bar{x}$ corresponds to $e: \tau \rightarrow \sigma$, let $M_{e}=\mathcal{F}_{\tau, \bar{y}}$, and if $(e: \tau \rightarrow \sigma) \leq\left(e^{\prime}: \tau^{\prime} \rightarrow \sigma\right)$ with $e=e^{\prime} \circ f$, we have a map $\varphi_{f}: M_{e} \rightarrow M_{e^{\prime}}$ induced by $\varphi_{f}: F_{S, \bar{s}}(f)^{-1}\left(\mathcal{F}_{\tau}\right) \rightarrow \mathcal{F}_{\tau^{\prime}}$. This gives a barycentric or polyhedral cochain complex $\left(C_{\mathrm{bct}}^{\bullet}, d_{\mathrm{bct}}\right)$ or $\left(C_{\mathrm{phd}}^{\bullet}, d_{\mathrm{phd}}\right)$ associated to the system $\left(M_{e}\right)_{e \in \mathscr{P}_{\sigma}}$. On the other hand, since $q_{\tau}: X_{\tau} \rightarrow X$ is always a finite map, it follows from [35], II Cor. 3.5, that the stalk of $q_{\tau *} \mathcal{F}_{\tau}$ at $\bar{x}$ is $\bigoplus_{\bar{y}} \mathcal{F}_{\tau, \bar{y}}$, where the sum is over all geometric points $\bar{y}$ of $X_{\tau}$ mapping to $\bar{x}$. From this one easily sees that the stalks of the complexes $\left(\mathscr{C}_{\text {bct }}^{\bullet}, d_{\text {bct }}\right)$ and $\left(\mathscr{C}_{\text {phd }}^{\bullet}, d_{\text {phd }}\right)$ at $\bar{x}$ coincide with the barycentric or polyhedral cochain complexes associated to $\left(M_{e}\right)$. Thus if for every point $\bar{x}$ of $X$ the system $\left(M_{e}\right)_{e \in \sigma}$ satisfies Condition (*) (keeping in mind $\sigma$ depends on $\bar{x}$ ), it follows that the complexes of sheaves $\mathscr{C}_{\text {bct }}^{\bullet}$ and $\mathscr{C}_{\text {phd }}^{\bullet}$ are exact. 
Example A.3. Take $\mathcal{F}_{\tau}=\mathcal{O}_{X_{\tau}}$, and let $\varphi_{e}=F_{S, \bar{s}}(e)^{*}$, the pull-back of functions via $F_{S, \bar{s}}(e)$. It is easy to check that Condition $(*)$ holds. Indeed, to each $e \in \mathscr{P}_{\sigma}$, we obtain a closed subscheme of $\operatorname{Spec} \mathcal{O}_{X, \bar{x}}$ given by the image of $\operatorname{Spec} \mathcal{O}_{X_{\tau}, \bar{y}} \stackrel{q_{\tau}}{\longrightarrow} \operatorname{Spec} \mathcal{O}_{X, \bar{x}}$ where $\bar{y}$ is the point of $X_{\tau}$ mapping to $\bar{x}$ corresponding to $e$. Then Condition $(*)$ is implied by the fact that given functions on some collection of these closed subschemes which agree on intersections, we can glue them to obtain a function on the union of these closed subschemes, and then extend to obtain an element of $\mathcal{O}_{X, \bar{x}}$. 


\section{Index of notations}

\section{1}

$M$
$N=M^{*}$

free abelian group of rank $n$

$M_{\mathbb{R}}, N_{\mathbb{R}}$

the dual group $N=\operatorname{Hom}(M, \mathbb{Z})$

$M \otimes_{\mathbb{Z}} \mathbb{R}, N \otimes_{\mathbb{Z}} \mathbb{R}$

$\operatorname{Aff}(M), \operatorname{Aff}\left(M_{\mathbb{R}}\right) \quad$ group of (integral) affine transformations of $M$

$B$

$\mathcal{T}_{B}$

$n$-dimensional topological manifold

$\pi: \tilde{B} \rightarrow B$

tangent bundle of $B$

$\delta: \tilde{B} \rightarrow M_{\mathbb{R}}$

universal covering

$\rho: \pi_{1}(B) \rightarrow \operatorname{Aff}\left(M_{\mathbb{R}}\right)$

$\Psi_{\gamma}$

Lin

developing map

Trans

$c_{\rho}$

holonomy representation

deck transformation given by $\gamma \in \pi_{1}(B)$

linear part of an affine transformation

translational part of an affine transformation

radiance obstruction of holonomy representation $\rho$

the flat connection on $\mathcal{T}_{B}$ induced from the affine

structure

$\Lambda_{\mathbb{R}}, \Lambda \quad$ local system of flat (integral) vector fields

$\check{\Lambda}_{\mathbb{R}}, \check{\Lambda}$

duals to $\Lambda_{\mathbb{R}}$ and $\Lambda$

$\mathcal{A} f f(B, \mathbb{R}), \mathcal{A f f}(B, \mathbb{Z})$ sheaf of (integral) affine functions on $B$

$g, K$

$\check{B}, \check{K}, \check{\delta}$

Hessian metric on $B$ and a local potential function

Legendre dual data

\section{2}

$\Delta \subset B$

discriminant locus

$i: B_{0} \rightarrow B$

$\Xi$

inclusion of complement of $\Delta$

reflexive lattice polytope

\section{3}

$\mathscr{P}$

$v, w$

$\sigma, \tau, \omega$

Int $\sigma$

$\exp _{v}: R_{v} \rightarrow B$

$\mathscr{P}_{v}$

$\tilde{\sigma} \rightarrow \sigma$

$S_{\sigma}$

$\mathscr{P}_{\max }$

$\operatorname{Bar}(\mathscr{P})$

$W_{\tau}$

$\mathscr{W}$

$\Delta^{\prime} \subset B$

polyhedral decomposition

vertices of $\mathscr{P}$

cells of $\mathscr{P}$

relative interior of $\sigma \in \mathscr{P}$

chart at vertex $v \in \mathscr{P} ; R_{v} \subset \Lambda_{\mathbb{R}, v}$

polyhedral decomposition of $R_{v}$

restriction of $\exp _{v}$ to lift $\tilde{\sigma} \in \mathscr{P}_{v}$ of $\sigma$

local submersion of neighbourhood of $\operatorname{Int}(\sigma)$

contracting $\sigma$

set of maximal cells of $\mathscr{P}$

(first) barycentric decomposition of $B$ wrt. $\mathscr{P}$

open star of barycenter of $\tau \in \mathscr{P}$ wrt. $\operatorname{Bar}(\mathscr{P})$

open covering $\left\{W_{\tau} \mid \tau \in \mathscr{P}\right\}$ of $B$

the maximal codimension two subcomplex of $\operatorname{Bar}(\mathscr{P})$

disjoint from vertices and from $\operatorname{Int}(\sigma)$ for all

$\sigma \in \mathscr{P}_{\max } ;$ contains $\Delta$.

$\Sigma_{v}$

fan in $\Lambda_{\mathbb{R}, v}$ induced from $\mathscr{P}$;

$\Lambda_{\mathscr{P}}, \Lambda_{\mathscr{P}, \mathbb{R}}$ generalizes to $\Sigma_{\tau}$ for $\tau \in \mathscr{P}$.

subsheaf of $\Lambda\left(\Lambda_{\mathbb{R}}\right)$ of vectors tangent to cells of $\mathscr{P}$ 


\begin{tabular}{|c|c|}
\hline$\Lambda_{\sigma}, \Lambda_{\sigma, \mathbb{R}}$ & stalk of $\Lambda_{\mathscr{P}}\left(\Lambda_{\mathscr{P}, \mathbb{R}}\right)$ at any $x \in \operatorname{Int}(\tau) \backslash \Delta$ \\
\hline $\mathcal{Q}_{\mathscr{P}}, \mathcal{Q}_{\mathscr{P}, \mathbb{R}}$ & $\begin{array}{l}\text { subsheaf of } i_{*}\left(\Lambda / \Lambda_{\mathscr{P}}\right) \text { of locally flat sections } \\
\text { and its realification }\end{array}$ \\
\hline $\mathcal{Q}_{\sigma}, \mathcal{Q}_{\sigma, \mathbb{R}}$ & stalk of $\mathcal{Q} \mathscr{P}\left(\mathcal{Q}_{\mathscr{P}}, \mathbb{R}\right)$ at any $x \in \operatorname{Int}(\tau) \backslash \Delta$ \\
\hline & $\begin{array}{l}\text { fan in } \mathcal{Q}_{\tau, \mathbb{R}} \text { defined by tangent wedges } K_{\sigma} \text { to } \sigma \in \mathscr{P} \\
\text { containing } \tau\end{array}$ \\
\hline$\underline{\operatorname{Cat}}(\mathscr{P})$ & $\begin{array}{l}\text { category with elements } \mathscr{P} \text { and morphisms given } \\
\text { by one-cells of Bar } \mathscr{P}\end{array}$ \\
\hline $\operatorname{Hom}(\tau, \sigma)$ & $\begin{array}{l}\text { morphisms in } \underline{\operatorname{Cat}}(\mathscr{P}) \text {; any } e: \tau \rightarrow \sigma \text { corresponds } \\
\text { uniquely to a face of } \tilde{\sigma}\end{array}$ \\
\hline$\Sigma(\tau)$ & quotient fan of fan $\Sigma$ by $\tau \in \Sigma$ \\
\hline$\tau^{-1} \Sigma$ & localization of fan $\Sigma: \tau^{-1} \Sigma=\{\sigma+\mathbb{R} \tau \mid \sigma \in \Sigma, \tau \subset \sigma\}$ \\
\hline & normal fan of $\tilde{\tau}$; fan in $\Lambda_{\tau, \mathbb{R}}^{*}$ \\
\hline $\mathcal{A f f}(B, \mathbb{R}), \mathcal{A f f}(B, \mathbb{Z})$ & $\begin{array}{l}\text { sheaf of continuous functions that are (integral) } \\
\text { affine on } B_{0}\end{array}$ \\
\hline $\begin{array}{l}\mathcal{P} \mathcal{L}_{\mathscr{P}}(B, \mathbb{R}) \\
\mathcal{P} \mathcal{L}_{\mathscr{P}}(B, \mathbb{Z})\end{array}$ & sheaf of (integral) piecewise linear functions on $B$ \\
\hline$\varphi, \varphi_{\sigma}$ & a piecewise linear function and the induced piecewise \\
\hline $\mathcal{M P} \mathcal{L}_{\mathscr{P}, \mathbb{R}}, \mathcal{M P} \mathcal{L}_{\mathscr{P}}$ & $\begin{array}{l}\text { sheaf of multivalued (integral) piecewise linear } \\
\text { functions }\end{array}$ \\
\hline & 1.4 \\
\hline$(\check{B}, \check{\mathscr{P}}, \check{\varphi})$ & Legendre transform of $(B, \mathscr{P}, \varphi)$ \\
\hline$\check{\sigma}$ & Legendre dual to $\sigma \in \mathscr{P}$; element of $\check{\mathscr{P}}$ \\
\hline$\Xi^{*}$ & dual of reflexive polytope $\Xi$ \\
\hline & 1.5 \\
\hline$d_{\omega}$ & generator of $\Lambda_{\omega}, \operatorname{dim} \omega=1$ \\
\hline$\check{d}_{\rho}$ & generator of $\mathcal{Q}_{\rho}^{*}, \operatorname{dim} \rho=n-1$ \\
\hline$v_{\omega}^{-}, v_{\omega}^{+}$ & vertices of $\omega$ ordered by $d_{\omega}$ \\
\hline$e_{\omega}^{-}, e_{\omega}^{+}$ & morphisms $v_{\omega}^{ \pm} \rightarrow \omega$ \\
\hline$\sigma_{\rho}^{-}, \sigma_{\rho}^{+}$ & $n$-dimensional cells adjacent to $\rho$; ordered by $\check{d}_{\rho}$ \\
\hline $\begin{array}{l}g_{\rho}^{-}, g_{\rho}^{+} \\
\gamma_{f_{1} f_{2}}^{e_{1} e_{2}}\end{array}$ & $\begin{array}{l}\text { morphisms } \rho \rightarrow \sigma_{\rho}^{ \pm} \\
\text {primitive loop distinguished by } f_{i}: v_{i} \rightarrow \tau \text { and }\end{array}$ \\
\hline $\begin{array}{l}T_{f_{1} f_{2}}^{e_{1} e_{2}} \\
T_{\omega}^{e_{1} e_{2}}\end{array}$ & $\begin{array}{l}g_{i}: \tau \rightarrow \sigma_{i} \\
\text { monodromy along } \gamma_{f_{1} f_{2}}^{e_{1} e_{2}} \\
T_{e_{\omega}^{+} e_{\omega}}^{e_{1} e_{2}} ; \text { monodromy around one-dimensional } \omega \in \mathscr{P} \\
\text { with respect to adjacent } \sigma_{1}, \sigma_{2} \in \mathscr{P}_{\max }\end{array}$ \\
\hline$n_{\omega}^{e_{1} e_{2}}$ & element of $\mathcal{Q}_{\omega}^{*}$ determining $T_{\omega}^{e_{1} e_{2}}$ \\
\hline $\begin{array}{l}T_{f_{1} f_{2}}^{\rho} \\
m_{f_{1} f_{2}}^{\rho}\end{array}$ & $\begin{array}{l}T_{f_{1} f_{2}}^{g_{\rho}^{+} g_{\rho}^{-}} ; \text {monodromy between } \sigma_{\rho}^{+}, \sigma_{\rho}^{-} \text {through } v_{1}, v_{2} \\
\text { element of } \Lambda_{\rho} \text { determining } T_{f_{1} f_{2}}^{\rho}\end{array}$ \\
\hline$T_{e}, e: \omega \rightarrow \rho$ & $\begin{array}{l}\text { distinguished primitive monodromy for } \operatorname{dim} \omega=1 \text {, } \\
\operatorname{dim} \rho=n-1\end{array}$ \\
\hline$n_{e}, m_{e}$ & multiples of $d_{\omega}$ and $\check{d}_{\rho}$, respectively, determining $T_{e}$ \\
\hline & PL-function on $\Sigma_{\omega}$ determined by $n_{\omega}^{e_{1} e_{2}}, e_{i}: \omega \rightarrow \sigma_{i}$ \\
\hline$\check{\psi}_{\rho}$ & PL-function on $\check{\Sigma}_{\rho}$ determined by $m_{f_{1} f_{2}}^{\rho}, f_{i}: v_{i} \rightarrow \rho$ \\
\hline
\end{tabular}




$$
\begin{aligned}
& \check{\Delta}(\omega) \subset \mathcal{Q}_{\omega, \mathbb{R}} \\
& \Delta(\rho) \subset \Lambda_{\rho, \mathbb{R}} \\
& \check{\Delta}_{e}(\tau) \subset \mathscr{\Delta}(\omega) \\
& \Delta_{e}(\tau) \subset \Delta(\rho) \\
& \mathscr{P}_{1}(\tau) \\
& \mathscr{P}_{n-1}(\tau) \\
& \check{\Delta}(\tau)
\end{aligned}
$$

$\Delta(\tau)$

$A$
$C(\sigma)$
$\check{P}_{\sigma}$
$R_{\sigma}$
$\check{X}_{\sigma}$
$\check{F}$
$\check{s}=\left(\check{s}_{e}\right)_{e: \rightarrow \sigma}$
$\check{F}_{A, \breve{s}}$
$\check{X}_{0}(B, \mathscr{P}, \check{s})$

$X(\Sigma)$

$X_{\sigma}$

$\sigma^{\vee}$

F

$W_{\tau_{1} \ldots \tau_{p}}$

$W_{e}$

$S$

$s=\left(s_{e}\right)_{e: \tau \rightarrow \sigma}$

$F_{S, s}$

$P_{\sigma}$

$\rho_{\sigma}$

$\partial P$

$V(\sigma) \subset U(\sigma)$

$\mathfrak{R}$

$\check{\omega}$

$U(\tau)$

$V(\tau)$

$\Phi_{\sigma_{1} \sigma_{2}}, \Phi_{e_{1} e_{2}}$

$K_{e}$

$V_{e}$

$P \check{M}(\tau)$

$s=\left(s_{e}\right)_{e: \tau \rightarrow \sigma}$ in the positive case, Newton polytope of $\psi_{\omega}$ in the positive case, Newton polytope of $\check{\psi}_{\rho}$ face determined by $e: \omega \rightarrow \tau$

face determined by $e: \tau \rightarrow \rho$ sets of morphisms $\{e: \omega \rightarrow \tau \mid \operatorname{dim} \omega=1\}$ sets of morphisms $\{f: \tau \rightarrow \rho \mid \operatorname{dim} \rho=n-1\}$ in the simple case, simplex built from $\check{\Delta}_{e}(\tau)$, $e \in \mathscr{P}_{1}(\tau)$ in the simple case, simplex built from $\Delta_{f}(\tau)$, $f \in \mathscr{P}_{n-1}(\tau)$

\section{1}

base ring

cone over $\sigma \in \mathscr{P}$ in $\Lambda_{\sigma, \mathbb{R}} \oplus \mathbb{R}$

$C(\sigma) \cap\left(\Lambda_{\sigma} \oplus \mathbb{Z}\right)$

$\mathbb{Z}\left[\check{P}_{\sigma}\right]$

$\operatorname{Proj} R_{\sigma}$

gluing functor for cone picture $\mathscr{P} \ni \sigma \mapsto R_{\sigma}$

gluing data for cone picture: $\check{s}_{e} \in \operatorname{Hom}\left(\check{P}_{\tau}, \mathbb{G}_{m}(A)\right)$

gluing functor for cone picture, twisted by $\check{s}$

$\operatorname{Proj}\left(\lim \breve{F}_{A, \check{s}}\right)$; glued scheme in cone picture

\section{2}

toric variety for fan $\Sigma$

$X\left(\Sigma_{\sigma}\right)$

dual of cone $\sigma$

gluing functor for fan picture $\mathscr{P} \ni \sigma \mapsto X_{\sigma}$ $W_{\tau_{1}} \cap \ldots \cap W_{\tau_{p}}$ connected component of $W_{\tau \sigma}$ selected by $e: \tau \rightarrow \sigma$ base scheme

closed gluing data: Čech 1-cocycle for $\mathcal{Q}_{\mathscr{P}} \otimes \mathbb{G}_{m}(S)$ wrt. $\mathscr{W}$; also: open gluing data, see below gluing functor for fan picture, twisted by $s$ $C(\sigma)^{\vee} \cap\left(\check{\Lambda}_{y} \oplus \mathbb{Z}\right)$

distinguished element of $P_{\sigma}$

$(\partial \sigma \cap N) \cup\{\infty\}$

$\operatorname{Spec} \mathbb{Z}\left[\partial P_{\sigma}\right] \subset \operatorname{Spec} \mathbb{Z}\left[P_{\sigma}\right]$

étale equivalence relation on $\coprod_{\sigma \in \mathscr{P}_{\max }} V(\sigma) \times S$

for $\omega \subset \sigma \in \mathscr{P}_{\max }$ corresponding face of $C(\sigma)^{\vee}$;

also, corresponding element of $\Sigma_{\sigma}$

$\operatorname{Spec} \mathbb{Z}\left[C(\tau)^{\vee} \cap(N \oplus \mathbb{Z})\right]$

toric boundary of $U(\tau)$

gluing isomorphisms

for $e: \tau \rightarrow \sigma$ the corresponding cone in $\Sigma_{\tau}$

$\operatorname{Spec} \mathbb{Z}\left[K_{e}^{\vee} \cap \mathcal{Q}_{\tau}^{*}\right](e: \tau \rightarrow \sigma)$

piecewise multiplicative functions on $\tilde{\tau}$;

equals $\Gamma\left(\tilde{\tau}, \pi^{*}\left(\mathcal{Q}_{\mathscr{P}} \otimes \mathbb{G}_{m}(S)\right)\right)$

open gluing data: $s_{e} \in P M(\tau)$ for $e: \tau \rightarrow \sigma$; 
also: closed gluing data, see above

$Z^{1}\left(\mathscr{P}, \mathcal{Q}_{\mathscr{P}} \otimes \mathbb{G}_{m}(S)\right)$ group of open gluing data

$B^{1}\left(\mathscr{P}, \mathcal{Q}_{\mathscr{P}} \otimes \mathbb{G}_{m}(S)\right)$ trivial open gluing data

$\bar{s}=\left(\bar{s}_{e}\right)_{e: \tau \rightarrow \sigma} \quad$ closed gluing data associated to open gluing data $s$

$\Phi_{e_{1} e_{2}}(s)$

$\mathfrak{R}_{e_{1} e_{2}}$

gluing isomorphism twisted by open gluing data $s$

$X_{0}(B, \mathscr{P}, s)$

$p_{\sigma}$

$q_{\tau}$

$\operatorname{Pic}^{\tau}(X)$

$\omega_{X}$

graph of $\Phi_{e_{1} e_{2}}$; component of $\mathfrak{R}$

glued algebraic space in fan picture:

$\bigsqcup_{\sigma \in \mathscr{P}_{\max }}(V(\sigma) \times S) / \mathfrak{R}$

for $\sigma \in \mathscr{P}_{\max }$ the morphism $V(\sigma \times S) \rightarrow X_{0}(B, \mathscr{P}, s)$

$X_{\tau} \times S \rightarrow X_{0}(B, \mathscr{P}, s)$

group of isomorphism classes of numerically trivial

line bundles on $X$

dualizing sheaf of $X$

\section{1}

$\alpha_{X}: \mathcal{M}_{X} \rightarrow \mathcal{O}_{X} \quad \log$ structure on $X$

$q: \mathcal{M}_{X} \rightarrow \overline{\mathcal{M}}_{X} \quad$ morphism to "ghost sheaf" $\overline{\mathcal{M}}_{X}=\mathcal{M}_{X} / \mathcal{O}_{X}$

$\mathcal{M}_{(X, D)}$

Spec $k^{\dagger}$

$\log$ structure on $X$ defined by divisor $D \subset X$

standard $\log$ point $\left(\operatorname{Spec} k, \mathbb{N} \oplus \mathbb{G}_{m}(k)\right)$

\section{2}

$P$

$\mathcal{R}$

$\rho, \bar{\rho}$

$X^{g}$

$\mathcal{L S}_{X^{g}}$

toric monoid; here: $P=\overline{\mathcal{M}}_{X, \bar{x}}$ for a fine $\log$

structure $\mathcal{M}_{X}$

local relation sheaf of $\overline{\mathcal{M}}_{X}$

section of $\mathcal{M}_{X}$ with $\alpha(\rho)=0$ and its image in $\overline{\mathcal{M}}_{X}$

space $X$ together with "ghost structure"

sheaf of germs of log-smooth structures of given ghost type; subsheaf of $\mathcal{E} x t^{1}\left(\overline{\mathcal{M}}_{X}^{\mathrm{gp}} / \bar{\rho}, \mathcal{O}_{X}^{\times}\right)$

\section{3}

V

$\mathcal{F}$

$\epsilon_{\tau}$

$s^{v}$

$s^{\omega}$

$D(s, \omega)$

$s^{h}$

$V(\sigma) \times \mathbb{G}_{m}^{r}=\operatorname{Spec} k\left[\partial P \oplus \mathbb{Z}^{r}\right]$

a subsheaf of $\mathcal{H o m}\left(\mathcal{R}, \mathcal{O}_{V}^{\times}\right)$mapping onto $\mathcal{L S}_{V}$

for a 2-face $\tau$ a cyclic choice of orientations of its edges germ of $s \in \Gamma\left(\tilde{\tau}, \pi^{*}\left(\mathcal{Q}_{\mathscr{P}} \otimes \mathbb{G}_{m}(k)\right)\right)$ at a vertex $v$.

$s^{v_{\omega}^{-}} / s^{v_{\omega}^{+}}$if $\operatorname{dim} \omega=1$ and $v_{\omega}^{ \pm} \rightarrow \omega$ are the two vertices

defined by $s^{\omega}=d_{\omega} \otimes D(s, \omega)$; global version: $D(s, e, f)$ generalization of $s^{v}$ for the case with self-intersections;

$h: v \rightarrow \tau$

$D(s, e, f), D(s, \omega, \sigma)$ for $e: \omega \rightarrow \tau, f: \tau \rightarrow \sigma, \operatorname{dim} \omega=1, \operatorname{dim} \sigma=n$, the element of $k^{\times}$defined by $s^{h^{-}} / s^{h^{+}}=d_{\omega} \otimes D(s, e, f)$

$\mathcal{N}_{\omega}$

line bundle on $X_{\omega}$ corresponding to the PL-function $\psi_{\omega}$ on $\Sigma_{\omega} ; \mathcal{L S}_{X_{0}(B, \mathscr{P}, s)} \subset \bigoplus_{\operatorname{dim} \omega=1} q_{\omega *} \mathcal{N}_{\omega}$

\section{1}

$\mathcal{S}$

base of toric degeneration: spectrum of discrete valuation $\operatorname{ring} R$

$\mathcal{X}$

total space of toric degeneration

generic fiber of toric degeneration 
$\mathcal{X}_{0}$

$\nu: \tilde{\mathcal{X}}_{0} \rightarrow \mathcal{X}_{0}$

$Z \subset \mathcal{X}, Z \subset X$

$X^{\dagger}=\left(X, \mathcal{M}_{X}\right)$

$\nu: \tilde{X} \rightarrow X$

$\underline{\operatorname{Cat}}(X)$

$\operatorname{Strata}(X)$

LPoly

$L P: \mathrm{C}$ central fiber of toric degeneration

normalization map

log-singular set; closed set of relative codimension two;

log Calabi-Yau space

normalization

category of toric strata of $X$

set of toric strata of $X$

category of lattice polytopes

$\rightarrow$ LPoly functor used to build dual intersection complex

\section{2}

$\mathcal{L} \quad$ (ample) line bundle on $X^{\dagger}$

$\varphi_{\mathcal{L}} \quad$ piecewise linear function on $B$ associated to $\mathcal{L}$

$\left(B, \mathscr{P}, \varphi_{\mathcal{L}}\right) \quad$ degeneration data for $\left(X^{\dagger}, \mathcal{L}\right)$ building on dual intersection complex of $X^{\dagger}$

$\left(\check{B}, \check{\mathscr{P}}, \check{\varphi}_{\mathcal{L}}\right)$

dual degeneration data for $\left(X^{\dagger}, \mathcal{L}\right)$ building on intersection complex of $X^{\dagger}$

4.3

$\mathcal{L} \mathcal{S}_{\text {pre }, X}^{+}$

$\bigoplus_{\operatorname{dim} \omega=1} q_{\omega *} \mathcal{N}_{\omega}$ where $\mathcal{N}_{\omega} \in \operatorname{Pic}\left(X_{\omega}\right)$ is as in $\S 3.3$

\section{1}

$Z_{i} \subset q_{\tau}^{-1}(Z)$

irreducible components of codimension 1 of $Z$ on $X_{\tau}$ $Z^{\prime} \subset q_{\tau}^{-1}(Z)$ higher codimension components of $Z$ on $X_{\tau}$

\section{2}

$H^{1}\left(X \backslash Z, \mathcal{M}_{X}^{\mathrm{gp}}\right) \quad$ logarithmic Picard group of $\left(X, \mathcal{M}_{X}\right)$

$\mathcal{M}_{\tau}, \overline{\mathcal{M}}_{\tau} \quad q_{\tau}^{*} \mathcal{M}_{X \backslash Z}, q_{\tau}^{-1} \overline{\mathcal{M}}_{X \backslash Z}$

$\mathscr{C}^{\bullet}$

(barycentric) resolution of $\mathcal{M}_{X \backslash Z}^{\mathrm{gp}}$;

$\mathscr{C}^{k}=\bigoplus_{\sigma_{0} \rightarrow \cdots \rightarrow \sigma_{k}} q_{\sigma_{k} *}\left(\mathcal{M}_{\sigma_{k}}^{\mathrm{gp}}\right)$

$\mathcal{I}^{\bullet}$

(barycentric) resolution of $\mathcal{O}_{X \backslash Z}^{\times}$;

$\mathcal{I}^{k}=\bigoplus_{\sigma_{0} \rightarrow \cdots \rightarrow \sigma_{k}} q_{\sigma_{k} *} \mathcal{O}_{X_{\sigma_{k}} \backslash q_{\sigma_{k}}^{-1}(Z)}$

(barycentric) resolution of $\overline{\mathcal{M}}_{X \backslash Z}^{\mathrm{gp}}$;

$\overline{\mathscr{C}}^{k}=\bigoplus_{\sigma_{0} \rightarrow \cdots \rightarrow \sigma_{k}} q_{\sigma_{k} *} \overline{\mathcal{M}}_{\sigma_{k}}^{\mathrm{gp}}$

$d_{\text {bct }}$

(barycentric) differential in $\mathscr{C}^{\bullet}, \mathcal{I}^{\bullet}$ etc.

$D_{\tau} \subset X_{\tau}$

toric boundary of $X_{\tau}$

$Q^{\bullet}$

$\Gamma\left(X \backslash Z, \mathscr{C}^{\bullet}\right) / \Gamma\left(X \backslash Z, \mathcal{I}^{\bullet}\right)$

cone in $\Sigma_{v}$ distinguished by $e: v \rightarrow \tau$

$\operatorname{PA}(e)$

$W_{\sigma_{0} \rightarrow \cdots \rightarrow \sigma_{p}}$

for $e: v \rightarrow \tau$, functions $\Lambda_{\mathbb{R}, v} \rightarrow \mathbb{R}$ piecewise affine wrt. $\tau_{e}^{-1} \Sigma_{v}$

connected component of $W_{\sigma_{0} \cdots \sigma_{p}}$ distinguished by

$\sigma_{0} \rightarrow \cdots \rightarrow \sigma_{p}$

$\lambda$

element of $\Gamma\left(X_{\tau} \backslash q_{\tau}^{-1}(Z), \overline{\mathcal{M}}_{\tau}^{\text {gp }}\right)$

$\lambda^{e} \quad$ for $e: v \rightarrow \tau$ image of $\lambda$ under isomorphism

$\check{D}(\lambda, e, f)$

$\Gamma\left(X_{\tau} \backslash q_{\tau}^{-1}(Z), \overline{\mathcal{M}}_{\tau}^{\mathrm{gp}}\right) \rightarrow \mathrm{PA}(e)$

for $e: v \rightarrow \tau$ the integer defined by $\check{D}(\lambda, e, f) \check{d}_{\rho}=$ 
$\lambda^{e-}-\lambda^{e+}$, where $\lambda^{e \pm} \in \operatorname{Aff}(B, \mathbb{Z})_{v}$ are the restrictions of $\lambda^{e}$ to the two maximal cones distinguished by $f: \tau \rightarrow \rho, \operatorname{codim} \rho=1$

\section{3}

$(\check{X}, \check{\mathcal{L}})$

$\Xi$

$\mathscr{P}$

$\mathscr{P}[k]$

$\left(M_{\tau}\right)$

$\varphi_{\sigma \tau}: M_{\tau} \rightarrow M_{\sigma}$ $\left(C_{\mathrm{bct}}^{\bullet}, d_{\mathrm{bct}}^{\bullet}\right)$

$\left(f_{\sigma_{0} \sigma_{1} \ldots \sigma_{k}}\right)_{\sigma_{0} \sigma_{1} \ldots \sigma_{k}}$

$\operatorname{sgn}(\tau, \omega)$

$\operatorname{sgn}\left(\left(\sigma_{0}, \ldots, \sigma_{k}\right), \sigma_{k}\right)$

$\left(C_{\text {phd }}^{\bullet}, d_{\text {phd }}^{\bullet}\right)$

$\left(f_{\tau}\right)_{\tau \in \mathscr{P}[k]}$

$\mathscr{P}_{\sigma}$

$\operatorname{sgn}\left(\left(\sigma_{0} \rightarrow \cdots\right.\right.$ $\left.\left.\rightarrow \sigma_{k}\right), \sigma_{k}\right)$

$\mathcal{F}_{\sigma}$

$\varphi_{e}$

$f_{\sigma_{0} \rightarrow \cdots \rightarrow \sigma_{k}}$
A.1

$d$-dimensional polytope

face poset of $\Xi$

set of $k$-dimensional faces of $\Xi$

system of abelian groups indexed by $\mathscr{P}$

homomorphism defined whenever $\tau \subset \sigma$

barycentric cochain complex for $\left(M_{\tau}\right)$;

$C_{\mathrm{bct}}^{k}=\bigoplus_{\sigma_{0} \sigma_{1} \ldots \sigma_{k}} M_{\sigma_{k}}$

$k$-dimensional simplicial cochain, i.e., element of $C_{\mathrm{bct}}^{k}$

\section{A.2}

for $\tau \subset \omega$ of codimension one sign comparing chosen orientations

sign comparing orientations of $\left(\sigma_{0}, \ldots, \sigma_{k}\right) \in \operatorname{Bar}(\mathscr{P})$

and of $\sigma_{k}$

polyhedral cochain complex for $\left\{M_{\tau}\right\}$;

$C_{\mathrm{bct}}^{k}=\bigoplus_{\tau \in \mathscr{P}[k]} M_{\tau}$

$k$-dimensional polyhedral cochain, i.e., element of $C_{\mathrm{phd}}^{k}$

\section{A.3}

on an integral affine manifold with polyhedral decomposition $\mathscr{P}$, the face poset of $\tilde{\sigma}$

global analogue of $\operatorname{sgn}\left(\left(\sigma_{0}, \ldots, \sigma_{k}\right), \sigma_{k}\right)$ in A.2

abelian sheaf on $X_{\sigma}$

gluing maps for $\left(\mathcal{F}_{\sigma}\right)$

global analogue of $f_{\sigma_{0} \sigma_{1} \ldots \sigma_{k}}$ in A.1 


\section{References}

[1] K. Altmann, The versal deformation of an isolated toric Gorenstein singularity, Invent. Math. 128 (1997) 443-479, MR 1452429, Zbl 0894.14025.

[2] M. Artin, Algebraization of formal moduli II: Existence of modifications, Ann. Math. 91 (1970) 88-135, MR 0260747, Zbl 0185.24701.

[3] M. Artin, A. Grothendieck, \& J.-L. Verdier, Théorie des topos et cohomologie étale des schémas (SGA 4), Lecture Notes in Mathematics, 269, 270, 305, Springer-Verlag, Berlin, 1971, MR 0354642, MR 0354643, MR 0354654, Zbl 0234.00007, Zbl 0237.00012, Zbl 0245.00002.

[4] P. Aspinwall \& D. Morrison, Chiral rings do not suffice: $N=(2,2)$ theories with nonzero fundamental group, Phys. Lett. B 334 (1994) 79-86, MR 1290072.

[5] O. Baues, Gluing affine 2-manifolds with polygons, Geom. Dedicata 75 (1999) 33-56, MR 1680196, Zbl 0953.57010.

[6] V. Batyrev, Dual polyhedra and mirror symmetry for Calabi-Yau hypersurfaces in toric varieties, J. Algebraic Geom. 3 (1994) 493-535, MR 1269718, Zbl 0829.14023.

[7] V. Batyrev \& L. Borisov, On Calabi-Yau complete intersections in toric varieties, in 'Higher-dimensional complex varieties' (Trento, 1994), 39-65, de Gruyter, Berlin, 1996, MR 1463173, Zbl 0908.14015.

[8] G. Bredon, Sheaf Theory, 2nd Edition, Springer-Verlag, 1997, MR 1481706, Zbl 0874.55001.

[9] S.-Y. Cheng \& S.-T. Yau, The real Monge-Ampère equation and affine flat structures, in 'Proceedings of the 1980 Beijing Symposium on Differential Geometry and Differential Equations', Vol. 1, 2, 3 (Beijing, 1980), 339-370, Science Press, Beijing, 1982, MR 0714338, Zbl 0517.35020.

[10] S. Chynoweth \& M. Sewell, Mesh duality and Legendre duality, Proc. R. Soc. Lond. A, 428 (1990) 351-377, MR 1050537, Zbl 0716.65102.

[11] F. DeMeyer, T. Ford, \& R. Miranda, The cohomological Brauer group of a toric variety, J. Algebraic Geom. 2 (1993) 137-154, MR 1185609, Zbl 0797.14017.

[12] R. Friedman, Global smoothings of varieties with normal crossings, Ann. Math. 118 75-114 (1983), MR 0707162, Zbl 0569.14002.

[13] W. Fulton, Introduction to toric varieties, Annals of Mathematics Studies, 131, Princeton University Press, Princeton, NJ, 1993, MR 1234037, Zbl 0813.14039.

[14] W. Goldman \& M. Hirsch, The radiance obstruction and parallel forms on affine manifolds, Trans. Amer. Math. Soc. 286 (1984) 629-649, MR 0760977, Zbl 0561.57014.

[15] M. Gross, Special Lagrangian Fibrations I: Topology, in 'Integrable Systems and Algebraic Geometry' (M.-H. Saito, Y. Shimizu \& K. Ueno, eds.), World Scientific, 1998, 156-193, MR 1672120, Zbl 0964.14033.

[16] _ Special Lagrangian Fibrations II: Geometry, in 'Surveys in Differential Geometry', Somerville, MA, International Press, 1999, 341-403, MR 1772274.

[17] , Topological Mirror Symmetry, Invent. Math. 144 (2001) 75-137, MR 1821145.

[18] , Toric Degenerations and Batyrev-Borisov Duality, Math. Ann. 333 (2005) 645-688.

[19] M. Gross \& B. Siebert, Affine manifolds, log structures, and mirror symmetry, Turkish J. Math. 27 (2003) 33-60, MR 1975331, Zbl 1063.14048. 
[20] - Torus fibrations and toric degenerations, in preparation.

[21] - Mirror symmetry via logarithmic degeneration data II, in preparation.

[22] A. Grothendieck \& J. Dieudonné, Eléments de Géométrie Algébrique, II, Publ. Math. IHES 8 (1961), MR 0163909, Zbl 0118.36206.

[23] C. Haase \& I. Zharkov, Integral affine structures on spheres and torus fibrations of Calabi-Yau toric hypersurfaces I, preprint, 2002, math.AG/0205321.

[24] N. Hitchin, The Moduli Space of Special Lagrangian Submanifolds, Ann. Scuola Norm. Sup. Pisa Cl. Sci. (4) 25 (1997) 503-515, MR 1655530, Zbl 1015.32022.

[25] L. Illusie, Logarithmic spaces (according to K. Kato), in 'Barsotti Symposium in Algebraic Geometry' (Abano Terme 1991), 183-203, Perspect. Math., 15, Academic Press, 1994, MR 1307397, Zbl 0832.14015.

[26] T. Kajiwara, Logarithmic compactification of the generalized Jacobian variety, J. Fac. Sci. Univ. Tokyo 40 (1993) 473-502, MR 1255052, Zbl 0817.14011.

[27] F. Kato, Log smooth deformation theory, Tohoku Math. J. 48 (1996) 317-354, MR 1404507.

[28] _ Logarithmic structures of Fontaine-Illusie, in 'Algebraic analysis, geometry, and number theory' (J.-I. Igusa et. al., eds.), 191-224, Johns Hopkins Univ. Press, Baltimore, 1989, MR 1463703, Zbl 0776.14004.

[29] Y. Kawamata \& Y. Namikawa, Logarithmic deformations of normal crossing varieties and smooothing of degenerate Calabi-Yau varieties, Invent. Math. 118 (1994) 395-409, MR 1296351, Zbl 0848.14004.

[30] D. Knutson, Algebraic Spaces, Lecture Notes in Math., 203, Springer-Verlag, Heidelberg, 1971, MR 0302647, Zbl 0221.14001.

[31] M. Kontsevich \& Y. Soibelman, Homological mirror symmetry and torus fibrations, in 'Symplectic geometry and mirror symmetry' (Seoul, 2000), 203-263, World Sci. Publishing, River Edge, NJ, 2001, MR 1882331.

[32] N.C. Leung, Mirror symmetry without corrections, Comm. Anal. Geom. 13 (2005) 287-331, MR 2154821.

[33] S. Mac Lane, Categories for the working mathematician, Graduate Texts in Mathematics, 5, Springer-Verlag 1971, MR 0354798, Zbl 0232.18001.

[34] G. Mikhalkin, Enumerative tropical algebraic geometry in $\mathbb{R}^{2}$, J. Amer. Math. Soc. 18 (2005), 313-377, MR 2137980.

[35] J.S. Milne, Étale Cohomology, Princeton University Press, 1980, MR 0559531, Zbl 0433.14012.

[36] T. Nishinou \& B. Siebert, Toric degenerations of toric varieties and tropical curves, preprint, 2004, math.AG/0409060, to appear in Duke Math. Journal.

[37] W. Niziol, Toric singularities: log-blow-ups and global resolutions, J. Algebraic Geom. 15 (2006) 1-29, MR2177194.

[38] T. Oda, Convex bodies and algebraic geometry. An introduction to the theory of toric varieties, Ergebnisse der Mathematik und ihrer Grenzgebiete (3), 15, Springer-Verlag, Berlin, 1988, MR 0966447, Zbl 0628.52002.

[39] A. Ogus, Logarithmic de Rham cohomology, preprint.

[40] M. Olsson, Semistable degenerations and period spaces for polarized $K 3$ surfaces, Duke Math. J. 125 (2004) 121-203, MR 2097359.

[41] M. Reid, Nonnormal del Pezzo surfaces, Publ. Res. Inst. Math. Sci. 30 (1994) 695-727, MR 1311389, Zbl 0867.14015. 
[42] W.-D. Ruan, Lagrangian torus fibration and mirror symmetry of Calabi-Yau hypersurface in toric variety, in 'Mirror Symmetry, IV' (Montreal, QC, 2000), 33-55, AMS/IP Stud. Adv. Math., 33, 2002, MR 1968216.

[43] S. Schröer \& B. Siebert, Irreducible degenerations of primary Kodaira surfaces, in 'Complex geometry' (Göttingen, 2000), 193-222, Springer-Verlag, 2002, MR 1922107, Zbl 1060.14053.

[44] - Toroidal crossings and logarithmic structures, preprint, 2002, math.AG/0211088, to appear in Adv. Math.

[45] B. Siebert, Log Gromov-Witten invariants, unfinished manuscript.

[46] H. Shima \& K. Yagi, Geometry of Hessian manifolds, Differential Geom. Appl. 7 (1997) 277-290, MR 1480540, Zbl 0910.53034.

[47] J. Steenbrink, Limits of Hodge structures, Inv. Math. 31 (1976) 229-257, MR 0429885, Zbl 0303.14002.

[48] A. Strominger, S.-T. Yau, \& E. Zaslow, Mirror Symmetry is T-duality, Nucl. Phys. B479 (1996) 243-259, MR 1429831, Zbl 0896.14024.

UCSD MATHEMATiCs 9500 Gilman Drive LA Jolla, CA 92093-0112

E-mail address: mgross@math.ucsd.edu

Mathematisches Institut Albert-Ludwigs-Universität Freiburg ECKERSTRASSE 1, 79104 FREIBURG GERMANY E-mail address: bernd.siebert@math.uni-freiburg.de 